\title{
UNTERSUCHUNG DER \\ ELEKTRONENDICHTE VON ANTIBIOTIKA IN \\ BEZUG AUF PHARMAKOLOGISCHE \\ WIRKSAMKEIT
}

\begin{abstract}
Dissertation zur Erlangung des mathematisch-naturwissenschaftlichen Doktorgrades (Doctor rerum naturalium)
\end{abstract}

Institut für Anorganische Chemie der Georg-August-Universität Göttingen

vorgelegt von

Julian Jacob Holstein

aus Berlin

Göttingen 2011 
Referent: Prof. George M. Sheldrick, FRS

Koreferent: Dr. habil. Birger Dittrich

Tag der Disputation: 9.9.2011 
Wem die Kunst das Leben ist, dessen Leben ist eine große Kunst.

Johann Sebastian Bach 


\section{Inhaltsverzeichnis}

$\begin{array}{ll}\text { Einleitung } & 1\end{array}$

I Grundlagen 3

1 Strukturbestimmung an Einkristallen 5

1.1 Röntgenbeugung . . . . . . . . . . . . . . . 5

1.2 Strukturlösung ..................... 6

1.3 Streufaktoren und Strukturfaktoren . . . . . . . . . . . . . 6

1.4 Auslenkungsparameter ................. 7

1.4.1 Hirshfeld-Test . . . . . . . . . . . . . . 8

1.4.2 Anharmonische Schwingung .......... 8

1.5 Verfeinerung ......................... 9

1.6 Absolute Struktur . . . . . . . . . . . . . . . . . . . 10

2 Modelle zur Beschreibung der Elektronendichte 13

2.1 Von der Wellenfunktion zur Elektronendichte . . . . . . . . . . 14

2.2 Von der Elektronendichte zum Atomformfaktor . . . . . . . . . . 15

2.3 Modell unabhängiger Atome . . . . . . . . . . . . . . . . . . 17

2.3.1 Überlagerung von Gauß-Funktionen . . . . . . . . . . . 18

2.3.2 Vergleich beider Formalismen von Atomformfaktoren . . . 19

2.4 Ladungsdichtestudien mit dem Multipolmodell . . . . . . . . . . . 19 
2.5 Transfer aus Datenbanken . . . . . . . . . . . 20

2.6 Quantenmechanische Modelle . . . . . . . . . . . . . 21

2.6.1 Verfeinerung mit Hirshfeld-Atomen . . . . . . . . . . . 22

2.6.2 Annäherung einer Wellenfunktion an experimentelle Daten 23

2.7 Vergleich der Modelle . . . . . . . . . . . . . . . . . . . . . . 24

3 Molekulare Eigenschaften $\quad 27$

3.1 Topologische Analyse der Elektronendichte . . . . . . . . . . . 27

3.2 Kritische Punkte . . . . . . . . . . . . . . 27

3.3 Laplacefunktion . . . . . . . . . . . . . . . . . . . . . . 29

3.4 Atomare Bassins . . . . . . . . . . . . . . . . . . . 29

3.5 Hirshfeld-Oberflächen . . . . . . . . . . . . . . . 30

3.6 Elektrostatisches Potential _............... . . 31

3.6.1 Politzer-Analyse . . . . . . . . . . . . . . . . 32

3.7 Stärke von Wasserstoffbrückenbindungen _ . . . . . . . . . . . 32

II Methoden und Experimente 35

4 Messplätze $\quad 37$

4.1 Australian Synchrotron _. . . . . . . . . . . . . 37

4.2 Deutsches Elektronen-Synchrotron (DESY) . . . . . . . . . . . 37

4.3 Swiss Light Source $(\mathrm{SLS}) \ldots \ldots$. . . . . . . . . . . . . 37

4.4 Messungen an der Universität Göttingen . . . . . . . . . . . . 38

5 Invariome $\quad 39$

5.1 Konzept der Bindungsordnung . . . . . . . . . . . . . . . . 39

5.2 Nomenklatur . . . . . . . . . . . . . . . . . . 41

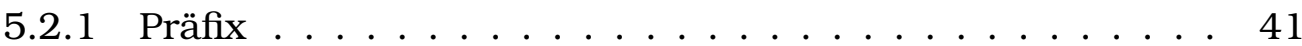

5.3 Ableiten von Modellverbindungen . . . . . . . . . . . . . 43 
5.4 Erstellen von Invariomen $\ldots \ldots . \ldots . \ldots 44$

5.4 .1 Geometrieoptimierung . . . . . . . . . . . . 44

5.4 .2 Theoretische Strukturfaktoren . . . . . . . . . . . 45

5.4 .3 Multipolprojektion ................ 45

5.5 Invariom-Datenbank . . . . . . . . . . . . . . . . 46

5.5 .1 Inhalt der Einträge $\ldots \ldots \ldots 46$

5.5 .2 Minimal-Kriterien . . . . . . . . . . . . . . . 46

5.6 Invariom-Transfer . . . . . . . . . . . . . . . 47

5.6 .1 InvariomTool . . . . . . . . . . . . . . 47

5.6 .2 Molecoolgt . . . . . . . . . . . . . . . 48

5.7 Vergleichsprüfung zur Transferierbarkeit . . . . . . . . . . . . 49

$\begin{array}{ll}\text { III Ergebnisse } & 51\end{array}$

6 Erweiterung der Invariom-Datenbank 53

6.1 Erkennung der Bindungsumgebung . . . . . . . . . . . . . 54

6.1 .1 Planarität . . . . . . . . . . . . . . . 54

6.1 .2 Aromatische Ringe . . . . . . . . . . . . 55

6.1 .3 Dreigliedrige Ringe . . . . . . . . . . . . . . . 57

6.2 Automatisierte Erzeugung von Invariomen . . . . . . . . . . . 58

6.2.1 Das Skript „setup_both“ . . . . . . . . . . . 58

6.3 Anwendung auf Heteroaromaten . . . . . . . . . . . . . . 59

6.3 .1 Nukleoside ................... 60

6.3 .2 Fluorochinolone ................. 63

7 Das Makrolid-Antibiotikum Roxithromycin 65

7.1 Pharmakologische Wirkung . . . . . . . . . . . . 66

7.2 Messung und Datenreduktion . . . . . . . . . . . 66 
7.3 Strukturelle Eigenschaften . . . . . . . . . . . . . 67

7.4 Sphärische Verfeinerung . . . . . . . . . . . . . . . . . 67

7.4.1 Positionen von Wasserstoffatomen . . . . . . . . . . . 68

7.5 Absolute Struktur . . . . . . . . . . . . . . . . . . . . 68

7.6 Invariom-Verfeinerung . . . . . . . . . . . . . . . . 69

7.6.1 Anisotrope Auslenkungsparameter . . . . . . . . . . . 70

7.7 Multipolverfeinerung . . . . . . . . . . . . . 71

7.8 Hybride Streufaktoren . . . . . . . . . . . . . 71

7.9 Validierung und Modellkorrektur . . . . . . . . . . . . . . 72

7.9.1 Differenzelektronendichte . . . . . . . . . . 72

7.9.2 Deformationselektronendichte . . . . . . . . 73

7.9.3 Gemischte Multipolverfeinerung (GMV) . . . . . . . . . 74

7.9.4 Quantifizierung der Fehlordnung . . . . . . . . . . . . 75

7.9.5 Restelektronendichte . . . . . . . . . . . 76

7.9.6 Anharmonische Schwingung . . . . . . . . . 76

7.10 Wasserstoffbrücken . . . . . . . . . . . . . 78

7.11 Elektrostatisches Potential _............... 79

7.11 .1 Einfluss der Verfeinerung . . . . . . . . . . . 79

7.11.2 Elektrostatisches Potential der molekularen Oberfläche . 80

7.12 Dipolmomente . . . . . . . . . . . . . . . . 82

7.13 Zusammenfassung ..................... 83

8 Fluorochinolon-Antibiotika $\quad 85$

8.1 Pharmakologische Wirkung . . . . . . . . . . . . 86

8.2 Asphärische Atomformfaktoren . . . . . . . . . . . . . . . 86

8.3 Strukturelle Eigenschaften . . . . . . . . . . . 87

8.4 Verwendete Datensätze . . . . . . . . . . . . . . . . . 97

8.5 Vorgehen zur Struktur-Modellierung . . . . . . . . . . . . . . 97 
8.6 Datenreduktion und Parametrisierung der Invariom-Verfeinerung 98

8.6.1 Ciprofloxacin $6 \mathrm{H}_{2} \mathrm{O} \ldots \ldots \ldots \ldots$

8.6.2 Ciprofloxacin $\cdot 1,4 \mathrm{H}_{2} \mathrm{O} \cdot \mathrm{HCl} \ldots \ldots \ldots 9$

8.6.3 Enoxacin $1,7 \mathrm{H}_{2} \mathrm{O} \ldots \ldots \ldots 0$

8.6.4 Enoxacin $3 \mathrm{H}_{2} \mathrm{O} \ldots \ldots \ldots$. . . . . . . . . . 100

8.6.5 Fleroxacin $\cdot \mathrm{H}_{2} \mathrm{O} \cdot \mathrm{HCl} \ldots \ldots \ldots$. . . . . . . . 100

8.6.6 Lomefloxacin $2 \mathrm{H}_{2} \mathrm{O} \cdot 0,95 \mathrm{Cl}^{-} \ldots \ldots \ldots 101$

8.6.7 Moxifloxacin· $0,5 \mathrm{H}_{2} \mathrm{O} \cdot 0,5 \mathrm{CH}_{3} \mathrm{OH} \cdot \mathrm{HCl} \ldots \ldots 101$

8.6.8 Nadifloxacin $0,5 \mathrm{H}_{2} \mathrm{O} \ldots \ldots \ldots 2$

8.6 .9 Norfloxacin-Anhydrat . . . . . . . . . . . . . 102

8.6.10 Norfloxacin $2 \mathrm{H}_{2} \mathrm{O} \ldots \ldots . \ldots 102$

8.6.11 Ofloxacin-Anhydrat . . . . . . . . . . . . 103

8.6.12 Sparfloxacin $3 \mathrm{H}_{2} \mathrm{O} \ldots \ldots . \ldots . \ldots . \ldots 103$

8.7 Verifizierung der Modellierung . . . . . . . . . . . . 108

8.7.1 Omit maps und Protonierungszustände . . . . . . . . . 108

8.7 .2 Gütefaktoren . . . . . . . . . . . . . . . 108

8.8 Elektrostatisches Potential _................. 112

8.8.1 Politzer-Analyse . . . . . . . . . . . . . . 114

8.9 Dipolmomente . . . . . . . . . . . . . . . 115

8.10 Vergleichsprüfung zur Transferierbarkeit . . . . . . . . . . . 117

8.10.1 Experimentelle Strukturfaktoren von Enoxacin $1,7 \mathrm{H}_{2} \mathrm{O} \quad \cdot 117$

8.10.2 Theoretische Strukturfaktoren der Fluorochinolone . . . . 118

8.11 Zusammenfassung . . . . . . . . . . . . . . . 123

Resümee

$\begin{array}{ll}\text { Summary } & 127\end{array}$

$\begin{array}{ll}\text { Literaturverzeichnis } & 129\end{array}$ 
A.1 Roxithromycin . . . . . . . . . . . . . . . . 138

A.1.1 Locale Koordinatensysteme, Hilfsatome und chemical contraints der gemischten Multipolverfeinerung (GMV). . . . . 138

A.1.2 Ergebnisse des Hishfeld-Tests (DMSDA) . . . . . . . . . 143

A.1.3 Bindungkritische Punkte . . . . . . . . . . . . . . . 145

A.1.4 Atomare Volumina und Ladungen aller Atome der Verfeinerungen von Roxithromycin . . . . . . . . . . . . 150

A.2 Invariome der Fluorochinolone . . . . . . . . . . . . . . . 154

Danksagung

Publikationsliste

Curriculum Vitae

179 


\section{Tabellenverzeichnis}

2.1 Atomzentrierte Modelle zur Beschreibung der Elektronendichte von Einkristall-Röntgenbeugungsdaten . . . . . . . . . . 25

3.1 Klassifizierung der kritischen Punkte nach ihren Krümmungsparametern ....................... 28

7.1 Kristallographische Daten von Roxithromycin . . . . . . . . . 66

7.2 Verfeinerungsergebnisse für Roxithromycin . . . . . . . . . . . . . 74

7.3 Atomare Ladungen und Volumina der von Rotationsfehlordnung betroffenen Gruppen Roxithromycins . . . . . . . . . . . 75

7.4 Bindungskritische Punkte von Wasserstoffbrückenbindungen . . 78

7.5 Energien der Wasserstoffbrückenbindungen . . . . . . . . . . 79

7.6 Politzer-Analyse des elektrostatischen Potentials auf der Vander-Waals-Oberfläche von Roxithromycin. . . . . . . . . . . . 82

7.7 Dipolmomente von Roxithromycin . . . . . . . . . . . . 83

8.1 Kristallographische Daten von Ciprofloxacin $6 \mathrm{H}_{2} \mathrm{O}$, Ciprofloxacin $\cdot 1,4$ $\mathrm{H}_{2} \mathrm{O} \cdot \mathrm{HCl}$, Enoxacin $1,7 \mathrm{H}_{2} \mathrm{O}$ und Lomefloxacin $2 \mathrm{H}_{2} \mathrm{O}$ 0,95. $\mathrm{Cl}^{-} \quad \cdot 104$

8.2 Kristallographische Daten von Nadifloxacin $0,5 \mathrm{H}_{2} \mathrm{O}$, NorflocaxinAnhydrat, Norflocaxin $2 \mathrm{H}_{2} \mathrm{O}$ und Ofloxacin-Anhydrat . . . . . 105

8.3 Messung und Verfeinerung von Ciprofloxacin $6 \mathrm{H}_{2} \mathrm{O}$ und Ciprofloxacin $\cdot 1,4$ $\mathrm{H}_{2} \mathrm{O} \cdot \mathrm{HCl} \ldots \ldots \ldots \ldots$. . . . . . . . . . . . . 109

8.4 Messung und Verfeinerung von Enoxacin 1 1, $7 \mathrm{H}_{2} \mathrm{O}$ und Enoxacin.3 $\mathrm{H}_{2} \mathrm{O} \ldots \ldots \ldots \ldots \ldots$ 
8.5 Messung und Verfeinerung von Fleroxacin $\cdot \mathrm{H}_{2} \mathrm{O} \mathrm{HCl}$ und Lomefloxacin 2 $\mathrm{H}_{2} \mathrm{O} 0,95 \cdot \mathrm{Cl}^{-} \ldots \ldots \ldots \ldots \ldots \ldots$

8.6 Messung und Verfeinerung von Norflocaxin. $2 \mathrm{H}_{2} \mathrm{O}$ und NorflocaxinAnhydrat . . . . . . . . . . . . . . . . 110

8.7 Messung und Verfeinerung von Sparfloxacin $3 \mathrm{H}_{2} \mathrm{O}$ und Moxifloxacin·0,5 $\mathrm{H}_{2} \mathrm{O} \cdot 0,5 \mathrm{CH}_{3} \mathrm{OH} \cdot \mathrm{HCl} \ldots \ldots \ldots \ldots . \ldots \ldots 11$

8.8 Messung und Verfeinerung von Nadifloxacin-0,5 $\mathrm{H}_{2} \mathrm{O}$ und OfloxacinAnhydrat . . . . . . . . . . . . . . . 111

8.9 Politzer-Analyse des elektrostatischen Potentials auf der Vander-Waals-Oberfläche der Fluorochinolone . . . . . . . . . . . 116

8.10 Dipolmomente der zwölf Fluorochinolone . . . . . . . . . . . . . 116

A. 1 Für Ciprofloxacin.6 $\mathrm{H}_{2} \mathrm{O}$ verwendete Invariome . . . . . . . . . 154

A.2 Für Ciprofloxacin $1,4 \mathrm{H}_{2} \mathrm{O} \cdot \mathrm{HCl}$ verwendete Invariome $\ldots \ldots . . .156$

A.3 Für Enoxacin $1,7 \mathrm{H}_{2} \mathrm{O}$ verwendete Invariome $\ldots \ldots \ldots$

A.4 Für Enoxacin.3 $\mathrm{H}_{2} \mathrm{O}$ verwendete Invariome $\ldots . . . . . .160$

A.5 Für Fleroxacin $\cdot \mathrm{H}_{2} \mathrm{O} \cdot \mathrm{HCl}$ verwendete Invariome $\ldots \ldots \ldots 16$

A.6 Für Lomefloxacin· $4 \mathrm{H}_{2} \mathrm{O} \cdot 0,95 \mathrm{Cl}^{-}$verwendete Invariome $\ldots . .162$

A.7 Für Moxifloxacin·0,5 $\mathrm{H}_{2} \mathrm{O} \cdot 0,5 \mathrm{CH}_{3} \mathrm{OH} \cdot \mathrm{HCl}$ verwendete Invariome 164

A.8 Für Nadifloxacin-0,5 $\mathrm{H}_{2} \mathrm{O}$ verwendete Invariome $\ldots . . . . . . .167$

A.9 Für Norfloxacin-Anhydrat verwendete Invariome . . . . . . . . . . 169

A.10 Für Norfloxacin $\cdot 2 \mathrm{H}_{2} \mathrm{O}$ verwendete Invariome $\ldots . . . . .170$

A.11 Für Ofloxacin-Anhydrat verwendete Invariome . . . . . . . . . 172

A.12 Für Sparfloxacin· $3 \mathrm{H}_{2} \mathrm{O}$ verwendete Invariome . . . . . . . . . . 173 


\section{Abbildungsverzeichnis}

1 Paul Ehrlich und die Struktur von Hexaphenylarsan auf der 200 DMBanknote ....................... 1

2.1 Die Wellenfunktion $\Psi_{1 s}$ des Wasserstoffatoms . . . . . . . . . 15

2.2 Die Elektronedichte $\rho_{1 s}$ des Wasserstoffatoms $\ldots \ldots \ldots$

2.3 Der Atomformfaktor des Wassertoffatoms . . . . . . . . . . . . . 17

2.4 Die Atomformfaktoren von $\mathrm{H}, \mathrm{C}, \mathrm{N}, \mathrm{O}, \mathrm{F}, \mathrm{Cl}$ und $\mathrm{Cl}^{-} \ldots \ldots$

2.5 Vergleich der Atomformfaktorkurven des Wasserstoffatoms . . . 19

5.1 Beispiele für absolute Konfiguration in Invariom-Namen . . . . . 42

5.2 Beispiel für Aromatizität in Invariom-Namen I . . . . . . . . . . . 42

5.3 Beispiel für Aromatizität in Invariom-Namen II . . . . . . . . . . . 43

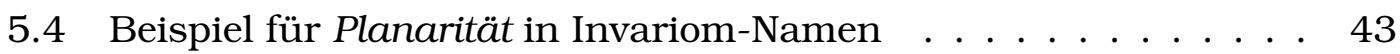

6.1 Erkennung aromatischer Ringe . . . . . . . . . . . . . . . 56

6.2 Die DNA Basenpaare AT und GC . . . . . . . . . . . . . 60

6.3 Modellverbindungen für Adenosin (A) . . . . . . . . . . . . . . 61

6.4 Modellverbindungen für Thymidin $(\mathrm{T}) \ldots \ldots$. . . . . . . . . 61

6.5 Modellverbindungen für Guanosin (G) . . . . . . . . . . . . . . 62

6.6 Modellverbindungen für Cytidin (C) . . . . . . . . . . . . . . 62

6.7 Modellverbindungen für Uridin (U) . . . . . . . . . . . . . . . . 63

7.1 Strukturformel von Roxithromycin . . . . . . . . . . . . . 67 
7.2 OMIT map von Roxithromycin . . . . . . . . . . . . . . . . 68

7.3 Deformationselektronendichte der Invariom-Verfeinerung . . . . 69

7.4 Anisotrope Auslenkungsparameter und lokale Koordinatenachsen 70

7.5 Hybride Streufaktoren von Wasserstoffatomen . . . . . . . . . . 72

7.6 Differenzelektronendichte aus EMV und IV . . . . . . . . . . 73

7.7 Deformationselektronendichte der Multipolverfeinerung . . . . . 74

7.8 Restelektronendichte der verschiedenen Modelle . . . . . . . . 77

7.9 Elektrostatisches Potential der Methoxygruppe von C71 _ . . . 80

7.10 Elektrostatisches Potential von Roxithromycin aus IV und GMV 81

7.11 Titelbild in der Zeitschrift Acta Crystallographica B im Jahr 201184

8.1 Strukturformeln der Fluorochinolone . . . . . . . . . . . . . . 89

8.2 Substitutionsmuster am Pharmakophor der neun untersuchten Fluorochinolone . . . . . . . . . . . . . . . 89

8.3 Berechnete Modellverbindungen für Ciprofloxacin . . . . . . . . 90

8.4 Berechnete Modellverbindungen für Enoxacin . . . . . . . . . . . 91

8.5 Berechnete Modellverbindungen für Fleroxacin . . . . . . . . . . . 92

8.6 Berechnete Modellverbindungen für Moxifloxacin . . . . . . . . . 93

8.7 Berechnete Modellverbindungen für Nadifloxacin . . . . . . . . . 94

8.8 Berechnete Modellverbindungen für Ofloxacin . . . . . . . . . . . 95

8.9 Berechnete Modellverbindungen für Sparfloxacin . . . . . . . . 96

8.10 OMIT map als Bestätigung der Wasserstoffatompositionen I . . 106

8.11 OMIT map als Bestätigung der Wasserstoffatompositionen II . . 107

8.12 Elektrostatisches Potential der ungeladenen Fluorochinolone . . 112

8.13 Elektrostatisches Potential der kationischen Fluorochinolone . . 113

8.14 Elektrostatisches Potential der zwitterionischen Fluorochinolone 114

8.15 Differenzelektronendichte von Enoxacin· $1,7 \mathrm{H}_{2} \mathrm{O} \ldots \ldots$

8.16 Differenzelektronendichte aus den Geometrieoptimierungen der ungeladenen Fluorochinolone . . . . . . . . . . . . . . . . 120 
8.17 Differenzelektronendichte aus den Geometrieoptimierungen der kationischer Fluorochinolone . . . . . . . . . . . . . . 121

8.18 Differenzelektronendichte aus den Geometrieoptimierungen der zwitterionischen Fluorochinolone . . . . . . . . . . . . . . . 122 


\section{Einleitung}

Im Jahr 1890 postulierte Paul Ehrlich die sog. Seitenketten-Theorie, nach der im Protoplasma Seitenketten (Chemorezeptoren) existieren, mit denen Arzneistoffe, die über eine „haptophore“ Gruppe verfügen eine chemische Bindung eingehen. Er spezifizierte, dass nur Stoffe, die eine geeignete reaktionsfähige „pharmakophore“ Gruppe sowie den passenden stereochemischen Bau besitzen, mit den Chemorezeptoren eine Bindung eingehen können. In Anlehnung an Emil Fischers Schlüssel-Schloß-Prinzip bezeichnete Ehrlich die Reaktion zwischen Arzneimittel und Rezeptor als „Schlüssel-Schloß-Theorie“. Er wurde damit zum Begründer der Rezeptortheorie, welche später zur Leitidee in der Arzneimittelforschung wurde. „In Anerkennung seiner Arbeit auf dem Gebiet der Immunologie“ erhielt Ehrlich zusammen mit Ilja Iljitsch Metschnikov 1908 den Nobelpreis für Medizin. Aufgrund der molekularen Flexibilität von Enzymen wird heute auch der Begriff der „induzierten Passform“ (engl. induced-fit) nach Koshland verwendet [1].

Zusammen mit Sahachiro Hata brachte Paul Ehrlich im Jahr 1910 das Arsphenamin als erstes Arzneimittel zur Behandlung der bakteriellen Infektionskrankheit Syphilis (Treponema pallidum) unter dem Namen Salvarsan auf den Markt, was den Auftakt chemotherapeutischer Behandlungen bildete [2]. $\mathrm{Zu}$ Ehren dieser Entdeckung wurde Ehrlich zusammen mit der molekularen Struktur von Hexaphenylarsan (in Anlehnung an Salvarsan) auf der 200 DM Banknote abgebildet. ${ }^{1}$

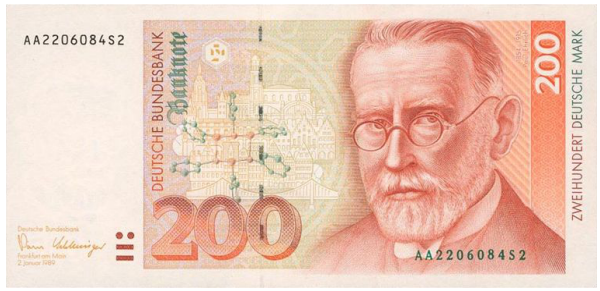

Abbildung 1: Paul Ehrlich und die Struktur von Hexaphenylarsan auf der 200 DM-Banknote.

Quelle: Wikimedia Commons

\footnotetext{
${ }^{1}$ Neuere Untersuchungen mit Elektrospray-Ionisation (ESI) Massenspektrometrie legen jedoch den Schluss nahe, dass es sich bei Salvarsan vorrangig um eine Mischung von Tri- und Pentameren handelt [3].
} 
Durch Resistenzentwicklungen von Bakterien gegen etablierte Breitband-Antibiotika befindet sich die antibakterielle Wirkstoffentwicklung in einem dauerhaften Wettlauf gegen die Zeit [4]. Mit der Charakterisierung bekannter Wirkstoffe soll in dieser Arbeit zum Verständnis intermolekularer Wechselwirkungen pharmakologisch aktiver Substanzen beigetragen werden. Als Basis wird dafür die Einkristall-Röntgenstrukturanalyse verwendet, welche durch technische Neuerungen, wie Flächendetektoren und intensive Strahlenquellen (Synchrotron) aber auch methodische Entwicklungen eine sehr leistungsfähige Methode geworden ist.

Grundlegende Aspekte und die verfügbaren Modelle werden im ersten Teil diskutiert. Der Informationsgehalt einer Strukturanalyse ist abhängig von Anzahl und Qualität der gesammelten Messwerte. Je höher die Auflösung, desto genauer können molekularen Eigenschaften charakterisiert werden. Als Beispiele seien die Bestimmung der absoluten Struktur und die Ermittlung der Aufenthaltsorte von Wasserstoffatomen genannt. Die sichere Bestimmung von Wasserstoffatompositionen sind zur Ableitung molekularer Eigenschaften von besonderer Bedeutung, da so der Protonierungszustand eines Moleküls im Kristallverband bestimmt werden kann.

Im zweiten Teil wird das Invariom-Konzept und die erweiterte Invariom-Nomenklatur vorgestellt. Der Invariom-Formalismus ermöglicht die Einbeziehung quantenmechanischen Berechnungen in die Modellierung der experimenteller Beobachtungen des Röntgenbeugungsexperiments und hat gegenüber der experimentellen Elektronendichtebestimmung den Vorteil, dass die molekulare Elektronendichteverteilung bereits bei atomarer Auflösung rekonstruiert werden kann.

Im dritten Teil wird das neu entwickelte Vorgehen zur Behandlung von $\mathrm{He}$ teroaromaten vorgestellt. Zur Bestimmung elektronischer Eigenschaften von neun antibiotisch wirksamen Fluorochinolinen und dem Makrolidantibiotikum Roxithromycin werden die asphärische Pseudoatome der Invariom-Datenbank auf die Kristallstrukturen transferiert und deren molekulare Eigenschaften berechnet. Im Gegensatz zu QSAR Methoden (engl. quantitative structureactivity relationship) [5], welche auf atomaren Punktladungen basieren, wird im Pseudoatom-Transfer die Deformation der Elektronendichte mit berücksichtigt. 


\section{Teil I}

Grundlagen 



\section{Kapitel 1}

\section{Strukturbestimmung an Einkristallen}

\subsection{Röntgenbeugung}

Aus der Wechselwirkung von monochromatischer Röntgenstrahlung mit kristalliner Materie lässt sich die dreidimensionale molekulare Struktur einer Probe bestimmen. Durch den elektrischen Feldvektor der Röntgenphotonen werden die Rumpf- und Valenzelektronen in Schwingung versetzt und re-emittieren ihrerseits Röntgenstrahlen derselben Wellenlänge. Dieser Vorgang der elastischen Streuung wird auch als Thompson-Streuung bezeichnet. Aufgrund der Periodizität des Kristalls entsteht durch konstruktive und destruktive Interferenz ein charakteristisches Beugungsbild aus diskreten Punkten. Auch wenn es sich bei dem beschriebenen physikalischen Phänomen nicht um Reflexion sondern um Beugung handelt, werden die Beugungspunkte als Reflexe bezeichnet. Um die Zusammenhänge zwischen Beugungspunkten und molekularer Struktur einfacher beschreiben zu können, bedient man sich einer mathematischen Hilfskonstruktion, dem reziproken Raum. Jeder Reflex des Beugungsbildes kann dem reziproken Gittervektor $\vec{h}$ einer Netzebene bzw. einem Tripel von Miller-Indizes h, k, l zugeordnet werden.

$$
\vec{h}=\mathbf{h} \overrightarrow{a^{*}}+\mathbf{k} \overrightarrow{b^{*}}+\mathbf{l} \vec{c}^{*}
$$

$\overrightarrow{a^{*}}, \overrightarrow{b^{*}}$ und $\vec{c}^{*}$ sind dabei die reziproken Basisvektoren des Gitters. Mit der Gleichung 1.2 nach Bragg kann die Auflösung eines Reflexes mit dem Beugungswinkel $\theta$ in $d$ oder $\sin \theta / \lambda$ umgerechnet werden.

$$
n \lambda=2 d \sin \theta
$$




\subsection{Strukturlösung}

Im Experiment wird für jeden Reflex eine Intensität $I(\vec{h})$ gemessen, welche proportional zum Guadrat der Strukturamplitude $|F(\vec{h})|$ ist.

$$
I(\vec{h}) \sim|F(\vec{h})|^{2}=F(\vec{h}) F^{*}(\vec{h})
$$

Der Strukturfaktor $F(\vec{h})$ setzt sich aus der Strukturamplitude $|F(\vec{h})|$ und dem Phasenwinkel $\Phi(\vec{h})$ zusammen. Dass der Phasenwinkel $\Phi(\vec{h})$ nicht gemessen werden kann, wird als Phasenproblem der Kristallographie bezeichnet, welches zur Strukturbestimmung überwunden werden muss.

$$
F(\vec{h})=|F(\vec{h})| \cdot e^{-i \Phi(\vec{h})}
$$

Bei atomarer Auflösung und Strukturen bis zu tausend Atomen können die Phasen der Reflexe mit direkten Methoden oder der Schweratommethode nach Patterson näherungsweise bestimmt werden.

\subsection{Streufaktoren und Strukturfaktoren}

Der Strukturfaktor $F(\vec{h})$ setzt sich als Summe der $N$ atomaren Streufaktoren zusammen. Der atomare Beitrag zum Strukturfaktor ist abhängig vom Atomort $r_{j}$, der in fraktionelle Koordinaten angegeben wird, dem Atomformfaktor $f_{j}$, welcher die Elektronendichte des Atoms repräsentiert und der Aufenthaltswahrscheinlichkeitsverteilung $T(\vec{h})$ des Atoms um die Atomposition.

$$
F(\vec{h})=\sum_{j=1}^{N} f_{j} \cdot e^{2 \pi i \vec{h} \vec{r}_{j}} \cdot T(\vec{h})
$$

Der Atomformfaktor $f_{j}$ ist abhängig vom Atomtyp, der verwendeten Wellenlänge und der Auflösung. Er setzt sich aus dem auflösungsabhängigen Term $f^{0}(\theta)$, dem realen Beitrag der anomalen Dispersion $\Delta f^{\prime}(\lambda)$ und dem imaginären Beitrag der anomalen Dispersion $\Delta f^{\prime \prime}(\lambda)$ zusammen. Der anomale Beitrag ist zwar abhängig von der verwendeten Wellenlänge aber unabhängig von der Auflösung.

$$
f(\theta, \lambda)=f^{0}(\theta)+\Delta f^{\prime}(\lambda)+i \Delta f^{\prime \prime}(\lambda)
$$


In der makromolekularen Kristallographie wird das anomale Signal zur Strukturlösung bei der experimentelle Bestimmung der Phasenwinkel verwendet. Es wird auch für die Bestimmung der absoluten Struktur von Kleinmolekülen verwendet (siehe Abschnitt 1.6).

Die Elektronendichte $\rho(\vec{r})$ kann nach Überwindung des Phasenproblems an einem beliebigen Punkt in der Elementarzelle durch Fourier-Synthese bzw. Fourier-Summation der Strukturfaktoren $F(\vec{h})$ als Observable des Röntgenbeugungsexperiments erhalten werden.

$$
\rho(\vec{r})=\frac{1}{V} \sum_{\vec{h}} F(\vec{h}) \cdot e^{-2 \pi i \vec{h} \vec{r}}
$$

\subsection{Auslenkungsparameter}

Für eine harmonischen Schwingung wird $T(\vec{h})$ gemäß Gleichung 1.8 verwendet.

$$
T_{\text {harm. }}(\vec{h})=e^{-B_{j}\left(\frac{\sin ^{2} \Theta}{\lambda^{2}}\right)}
$$

Im einfachsten Falle wird der Debye-Waller-Faktor ${ }^{1} B_{j}$ isotrop verwendet. Dabei ist $\bar{u}_{j}$ die Schwingungsamplitude der harmonischen Schwingung.

$$
B_{j}=8 \pi^{2} U_{j}=8 \pi^{2} \bar{u}_{j}^{2}
$$

Bei atomarer Auflösung wird meist zusätzlich mit einem symmetrischen Tensors $U_{i j}$ die Anisotropie der atomaren Schwingung als Ellipsoid abgebildet. Dafür werden die sechs Koeffizienten des zweistufigen Tensors $\left(U_{11}, U_{12}, U_{13}, U_{22}\right.$, $U_{23}$ und $U_{33}$ ) verfeinert, also pro Atom sechs Parameter benötigt. Da der DebyeWaller-Faktor auch Fehlordnung oder Mosaizität des Kristalls kompensieren kann, wird allgemein von anisotropen Auslenkungsparametern (engl. anisotropic displacement parameter, ADP) gesprochen.

\footnotetext{
${ }^{1}$ In den Lehrbüchern [6, 7] wird $B_{j}$ als Debye-Waller-Faktor bezeichnet. Die Autoren aktuellerer Literatur zu diesem Thema $[8,9]$ hingegen meinen mit dem Debye-Waller-Faktor $T(\vec{h})$.
} 


\subsubsection{Hirshfeld-Test}

Der Hirshfeld-Test basiert auf der Annahme, dass sich die Schwingungen der Atome im Kristall in zwei Energiebereiche aufteilen lassen [10]: Die externen und die internen Schwingungen. Mit der Näherung, dass die internen Schwingungen dabei vernachlässigbar klein sind, kann geschlussfolgert werden, dass die Amplituden der Auslenkung von zwei kovalent gebundenen Atomen A und $\mathrm{B}$ ähnlicher Masse in Bindungsrichtung gleich sind [11].

$$
\Delta_{A, B}=z_{A}^{2}-z_{B}^{2} \sim 0
$$

Dabei sind $z_{A}^{2}$ und $z_{B}^{2}$ die Beiträge der $U_{i j}$ Parameter von Atom A und B in Bindungsrichtung. Die Werte werden üblicherweise als Differenz $\Delta_{A, B}$ angegeben und sollten nicht höher als $1,0 \cdot 10^{-3} \AA^{2}$ sein. Es wird auch die vom englischen Begriff „Differences of Mean-Squares Displacement Amplitudes“ stammende Abkürzung DMSDA verwendet.

\subsubsection{Anharmonische Schwingung}

Wenn die harmonische Näherung nicht ausreicht um die Aufenthaltswahrscheinlichkeitsverteilung eines Atoms zu beschreiben, können bei subatomar aufgelösten Datensätzen zusätzlich die Koeffizienten der Gram-Charlier-Expansion dritter und vierter Ordnung verfeinert werden: Dadurch wird Aufenthaltswahrscheinlichkeitsverteilung als anharmonische Schwingungen beschrieben. Für die dritte Ordnung werden dabei die 10 Koeffizienten $C_{j k l}$ eines dreistufigen Tensors und für die vierte Ordnung 15 Koeffizienten $D_{j k l m}$ eines vierstufigen Tensors als zusätzliche Parameter pro Atom verfeinert. Die anharmonische Schwingung $T_{G C}(\vec{h})$ ist dabei nach Gleichung 1.11 definiert.

$$
\begin{aligned}
T_{G C}(\vec{h})=T_{\text {harm }}(\vec{h})\left[1+\frac{(2 \pi i)^{3}}{3 !} \cdot \sum_{j=1}^{3} \sum_{k=1}^{3} \sum_{l=1}^{3} C_{j k l} h_{j} h_{k} h_{l}+\right. \\
\left.\frac{(2 \pi i)^{4}}{4 !} \cdot \sum_{j=1}^{3} \sum_{k=1}^{3} \sum_{l=1}^{3} \sum_{m=1}^{3} D_{j k l m} h_{j} h_{k} h_{l} h_{m}\right]
\end{aligned}
$$

Der dreistufige Tensor wird in Gleichung 1.12 ausgeschrieben. Aufgrund der Symmetrie des Tensors können dabei identische Elemente zusammen gefasst werden. Der Faktor vor den jeweiligen Termen ist die Multiplizität, die aus der Permutation von $h_{j}, h_{k}$ und $h_{l}$ resultiert. 


$$
\begin{aligned}
\sum_{j=1}^{3} \sum_{k=1}^{3} \sum_{l=1}^{3} C_{j k l} h_{j} h_{k} h_{l}= & C_{111}\left(h_{1}\right)^{3}+3 C_{112}\left(h_{1}\right)^{2} h_{2}+3 C_{113}\left(h_{1}\right)^{2} h_{3}+ \\
& 3 C_{122} h_{1}\left(h_{2}\right)^{2}+6 C_{123} h_{1} h_{2} h_{3}+3 C_{133} h_{1}\left(h_{3}\right)^{2}+ \\
& C_{222}\left(h_{2}\right)^{3}+3 C_{223}\left(h_{2}\right)^{2} h_{3}+3 C_{233} h_{2}\left(h_{3}\right)^{2}+ \\
& C_{333}\left(h_{3}\right)^{3}
\end{aligned}
$$

$h_{1}, h_{2}, h_{3}$ sind gemäß Gleichung 1.13 das Produkt aus Miller-Indizes und den reziproken Basisvektoren des Gitters.

$$
\begin{aligned}
& h_{1}=\mathbf{h} \cdot \vec{a}^{*} \\
& h_{2}=\mathbf{k} \cdot \vec{b}^{*} \\
& h_{3}=\mathbf{l} \cdot \vec{c}^{*}
\end{aligned}
$$

\subsection{Verfeinerung}

Das Ziel jeder Verfeinerung ist die Minimierung der Abweichungen von gemessenen Strukturfaktoren $F_{o}(\vec{h})$ und den Strukturfaktoren des verwendeten Modells $F_{c}(\vec{h})$. Zur Berechnung der Strukturfaktoren aus den Strukturamplituden werden dafür die Phasenwinkel $\Phi(\vec{h})$ des Strukturmodells verwendet. Die Verfeinerung erfolgt mit der „Methode der kleinsten Guadrate“ nach Carl Friedrich Gauß [12], wobei in der Kristallographie entweder $F(\vec{h})$ oder $F^{2}(\vec{h})$ minimiert werden kann. Beide Varianten sind in Gleichung 1.14 angegeben.

$$
\begin{gathered}
\text { Minimierung von } F(\vec{h})=\sum_{\vec{h}} w(\vec{h})\left[F_{o}(\vec{h})-k F_{c}(\vec{h})\right]^{2} \\
\text { Minimierung von } F^{2}(\vec{h})=\sum_{\vec{h}} w(\vec{h})\left[F_{o}^{2}(\vec{h})-k F_{c}^{2}(\vec{h})\right]^{2}
\end{gathered}
$$

Dabei ist $k$ der relative Skalierungsfaktor und $w(\vec{h})$ steht für die Gewichtungsfunktion, welche auf zwei verschiedene Weisen verwendet wird. Nach Gleichung 1.15 ist die Gewichtungsfunktion nur abhängig von der Standardabweichung $\sigma\left[F_{o}^{2}(\vec{h})\right]$ der gemessenen Strukturfaktoren.

$$
w(\vec{h})=\frac{1}{\sigma^{2}\left[F_{o}^{2}(\vec{h})\right]}
$$


Im Zwei-Parameter-Gewichtungsschema nach Gleichung 1.16 werden über $P$ zusätzlich $F_{o}(\vec{h})$ und $F_{c}(\vec{h})$ gemittelt mit einbezogen. Die beiden Parameter des Gewichtungsschemas sind die Multiplikatoren a und b.

$$
w(\vec{h})=\frac{1}{\sigma^{2}\left[F_{o}^{2}(\vec{h})\right]+(\mathbf{a} P)^{2}+\mathbf{b} P} \text { mit } P=\frac{2 F_{c}^{2}(\vec{h})+F_{o}^{2}(\vec{h})}{3}
$$

Mit den statistischen Gütefaktoren (engl. figures of merit, FOM) kann am Ende der Verfeinerung die Übereinstimmung zwischen den gemessenen Daten und dem Modell quantifiziert werden. Der Residualindex $R(F)$ nach Gleichung 1.17 sollte für gute Strukturen unterhalb von $5 \%$ liegen. Der $R(F)$ wird auch $R$-Wert oder $R_{1}$ genannt.

$$
R(F)=\frac{\sum_{\vec{h}}|| F_{o}(\vec{h})|-| F_{c}(\vec{h})||}{\sum_{\vec{h}}\left|F_{o}(\vec{h})\right|}
$$

Die Anpassungsgüte $S$ (engl. goodness of fit, Goof) nach Gleichung 1.18 sollte idealerweise bei „1“ liegen, was bedeutet, dass das Modell die Daten im Rahmen der Standardabweichungen beschreibt. Neben den Strukturfaktoren geht hier auch die Anzahl an Reflexen $n_{\text {ref }}$ und Parametern $n_{\text {par }}$ ein. Bei Verwendung der zwei-Parameter-Gewichtung kann $S$ jedoch nicht als unabhängiges Gütekriterium verwendet werden, da die Gewichtungsparameter a und b so angepasst werden, dass $S$ einen Wert von 1 annimmt.

$$
S=\text { Goof }=\sqrt{\sum \frac{w(\vec{h})\left[F_{o}^{2}(\vec{h})-F_{c}^{2}(\vec{h})\right]^{2}}{n_{\mathrm{ref}}-n_{\mathrm{par}}}}
$$

Die Restelektronendichte $\Delta \rho(\vec{r})$ (Gleichung 1.19) sollte nach erfolgreicher Modellierung gering und gleichmäßig verteilt sein.

$$
\Delta \rho(\vec{r})=\frac{1}{V} \sum_{\vec{h}}\left[\left|F_{o}(\vec{h})\right|-\left|F_{c}(\vec{h})\right|\right] \cdot e^{i \Phi(\vec{h})} \cdot e^{-2 \pi \vec{h} \vec{r}}
$$

\subsection{Absolute Struktur}

Die Bestimmung der absoluten Struktur ist besonders im Hinblick auf Wirkstoffentwicklung, aber auch in vielen chemischen Fragestellungen von großer 
Bedeutung. Mit Hilfe des Zwillingsgesetzes entwickelte H. D. Flack ein Verfahren, um die absolute Struktur zu bestimmen [13]. Dafür wurde der Parameter $x$ eingeführt, welcher die enantiomorphe Polarität definiert. (engl. enantiomorph polarity defining parameter). $x$ wird auch als Flack-Parameter bezeichnet und ist gemäß Gleichung 1.20 definiert.

$$
F_{c}^{2}(\vec{h}, x)=(1-x) \cdot F_{c}^{2}(\vec{h})+x \cdot F_{c}^{2}(-\vec{h})
$$

Dabei werden die Unterschiede der Friedel-Paare $F_{c}^{2}(\vec{h})$ und $F_{c}^{2}(-\vec{h})$ ausgenutzt, welche auf die Beiträge aus der anomalen Dispersion zurückzuführen sind. Die Unterschiede der Friedel-Paare werden auch als Bijvoet-Differenzen [14] bezeichnet.

Die Methode nach S. Parsons basiert auf der Arbeit von H. D. Flack. Allerdings wird $Q_{c}(\vec{h})$ anstelle von $F_{c}^{2}(\vec{h})$ verwendet, wodurch eine Art Gewichtung eingeführt wird. Diese Methode sorgt für eine deutlich kleinere Standardabweichung des Flack-Parameters $x$ und eignet sich deshalb noch besser für die sichere Bestimmung der absoluten Struktur. Sie wurde erstmals auf dem „European Crystallographic Meeting (ECM)“ im Jahr 2004 vorgestellt [15] und ist in den Programmen XPREP (Bruker AXS) und CRYSTALS [16] implementiert. $Q_{c}(\vec{h})$ ist gemäß Gleichung 1.21 definiert.

$$
Q_{c}(\vec{h})=\frac{F_{c}^{2}(\vec{h})-F_{c}^{2}(-\vec{h})}{F_{c}^{2}(\vec{h})+F_{c}^{2}(-\vec{h})}
$$

Weitere grundlegende Aspekte der konventionellen Röntgenstrukturanalyse von Einkristallen werden ausführlich in Lehrbüchern sowohl für Kleinmoleküle [6, 7] als auch für Makromoleküle [17] beschrieben und sollen hier nicht weiter im Detail erläutert werden. 


\section{Kapitel 2}

\section{Modelle zur Beschreibung der Elektronendichte}

Die Elektronendichte ist nach Überwindung des Phasenproblems die Observable des Einkristall-Röntgenbeugungsexperiments. Besonders bei subatomar aufgelösten Datensätzen mit $d \leq 0,5 \AA$ bzw. $\frac{\sin \theta}{\lambda} \geq 1,0 \AA^{-1}$ spielen die im Strukturmodell zur Beschreibung der Elektronendichte verwendeten Atomformfaktoren $f_{j}$ deshalb eine wichtige Rolle. In der Röntgenstrukturanalyse stehen dafür eine Reihe von atomzentrierten Modellen zur Verfügung. Zum konventionellen Modell unabhängiger Atome gibt es mittlerweile zahlreiche Alternativen. So wird heute für experimentelle Ladungsdichtestudien das Multipolmodell am häufigsten verwendet. Damit unmittelbar verknüpft sind die asphärischen Datenbanken, von denen die Invariom-Datenbank eine zentrale Rolle dieser Arbeit einnimmt. Zusätzlich werden zwei neue und vielversprechende quantenchemischen Methoden einleitend vorgestellt, auch wenn sie in dieser Arbeit keine Anwendung finden.

Auf das Entropiemaximum-Verfahren (engl. maximum entropy method, MEM) [18] wird hier nicht näher eingegangen, da dort bis auf einen Startwert modellfrei gearbeitet wird. Dass dadurch auf die Entfaltung von Elektronendichte und thermischer Bewegung verzichtet wird, kann als Vor-oder Nachteil der Methode ausgelegt werden. MEM-Berechnungen werden u.a. in der Gruppe von Prof. Sander van Smaalen durchgeführt [19, 20].

Einleitend soll der Zusammenhang zwischen quantenchemischen Wellenfunktionen und Atomformfaktoren anhand des Wasserstoffatoms diskutiert werden. 


\subsection{Von der Wellenfunktion zur Elektronendichte}

Die zur Beschreibung der Elektronendichte verwendeten radialen Atomformfaktoren entspringen theoretischen Überlegungen. So kann die SchrödingerGleichung $E \cdot \Psi=\hat{\mathrm{H}} \Psi$ im Fall des Wasserstoffatoms exakt gelöst werden. Der Hamilton-Operator $\hat{\mathrm{H}}$ setzt sich in diesem Fall aus der doppelten partiellen Ortsableitung in den drei Raumrichtungen $\mathrm{x}, \mathrm{y}, \mathrm{z}$ und der potentiellen Energie $E_{\text {pot }}$ zusammen. In Gleichung 2.1 wird der Hamilton-Operator in kartesischen Koordinaten angegeben. Die Potentielle Energie $E_{\text {pot }}$ resultiert aus der Coulomb'schen Anziehungskraft vom Elektron und dem Proton des Kerns.

$$
\begin{aligned}
& E \cdot \Psi=-\frac{\hbar}{2 m} \nabla^{2} \Psi+E_{\mathrm{pot}} \Psi \\
& E \cdot \Psi=-\frac{\hbar}{2 m}\left(\frac{\partial^{2} \Psi}{\partial x^{2}}+\frac{\partial^{2} \Psi}{\partial y^{2}}+\frac{\partial^{2} \Psi}{\partial z^{2}}\right)+\frac{e^{2}}{4 \pi \epsilon_{0} r} \Psi
\end{aligned}
$$

In Polarkoordinaten setzt sich die Wellenfunktion $\Psi(r, \vartheta, \varphi)$ in Abhängigkeit der Haupt-, Neben- und Magnetquantenzahlen $(n, l$ und $m$ ) allgemein aus dem Normierungsfaktor für den radialen Term $N_{n, l}$, der radialen Eigenfunktion $R_{n, l}(r)$ und der normierten Kugelflächenfunktion $Y_{l}^{m}(\vartheta, \varphi)$ zusammen.

$$
\Psi(r, \vartheta, \varphi)=N_{n, l} \cdot R_{n, l}(r) \cdot Y_{l}^{m}(\vartheta, \varphi)
$$

Für alle weiteren Überlegungen wird der Radius $r$ an dieser Stelle in Abhängigkeit des ersten Bohrschen Radius ${ }^{1}\left(a_{0}\right)$ und des Kontraktionsparameters $\kappa$ definiert. $\mathrm{x}$ ist dabei der Abstand zum Atomkern.

$$
r=\frac{\kappa}{a_{0}} \cdot \mathbf{x}
$$

Auch wenn im Fall des isolierten Wasserstoffatoms $\kappa=1,0$ ist, soll es hier schon mit eingeführt und im folgenden als $\kappa$-Formalismus weiter verwendet werden.

Für das $1 s$-Orbital des Wasserstoffatoms lautet die Wellenfunktion

$$
\Psi_{1 s}=\frac{1}{\sqrt{\pi}} e^{-r}
$$

und das Betragsquadrat der Wellenfunktion

$$
\left|\Psi_{1 s}\right|^{2}=\frac{1}{\pi} e^{-2 r} .
$$

${ }^{1} a_{0}=0,52917721092 \AA$ 
Beide Funktionen sind in Abbildung 2.1 aufgetragen. Detaillierte Herleitungen der Wellenfunktion sind in Lehrbüchern der Physik [21] oder physikalischen Chemie [22] zu finden. Da die Wellenfunktion keine anschauliche Bedeutung hat, soll zusätzlich die Aufenthaltswahrscheinlichkeitsdichte $\rho(r)$ des Elektrons gegen den Radius aufgetragen werden.

$$
\rho(r)=4 \pi r^{2}\left|\Psi_{n, l}\right|^{2}
$$

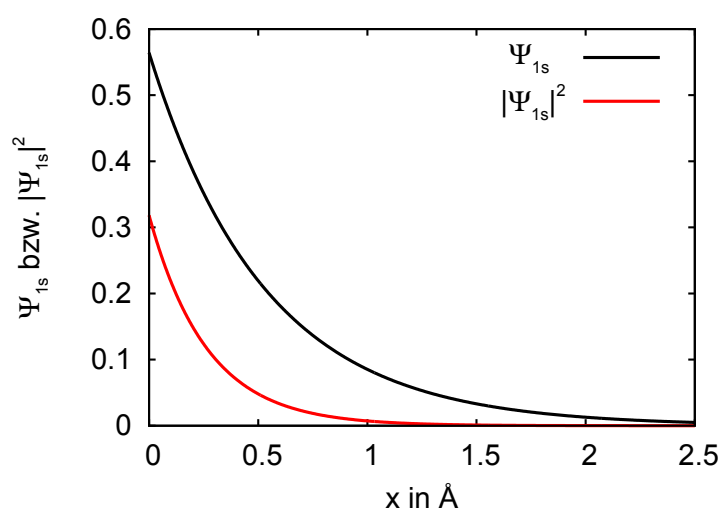

Abbildung 2.1: Die Wellenfunktion $\Psi_{1 s}$ und das Betragsquadrat der Wellenfunktion $\left|\Psi_{1 s}\right|^{2}$ des Wasserstoffatoms.

Für das $1 s$-Orbital des Wasserstoffatoms folgt daraus

$$
\rho_{1 s}(r)=4 r^{2} e^{-2 r} .
$$

Der radiale Verlauf der Aufenthaltswahrscheinlichkeitsdichte des $1 s$ Orbitals weist ein Maximum im Abstand des ersten Borschen Radius auf. In diesem Abstand zum Kern hält sich das Elektron des $1 s$ Orbitals im Durchschnitt am häufigsten auf.

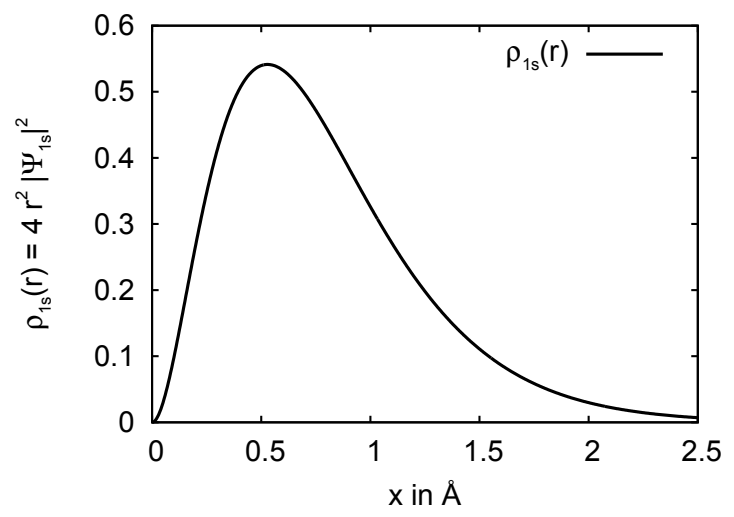

Abbildung 2.2: Die Elektronedichte $\rho_{1 s}$ des Wasserstoffatoms.

\subsection{Von der Elektronendichte zum Atomformfaktor}

Aus der Elektronendichte können die Atomformfaktoren abgeleitet werden. Der Atomformfaktor $f_{j}\left(r^{*}\right)$, welcher ein Konstrukt des reziproken Raumes ist, lässt sich durch Fouriertransformation der atomaren Elektronendichte $\rho_{j}(r)$ berechnen. Dabei steht $r$ für den Ortsvektor im realen Raum und $r^{*}$ für den Vektor im reziproken Raum. Da Radialfunktionen nur abhängig vom Abstand sind, handelt es sich hier um den eindimensionalen Fall, für den gilt:

$$
\begin{aligned}
& f_{j}\left(r^{*}\right)=\mathcal{F} \mathcal{T}\left[\rho_{j}(r)\right] \\
& f_{j}\left(r^{*}\right)=\int_{-\infty}^{\infty} \rho_{j}(r) \cdot e^{2 \pi i r^{*} r} d r
\end{aligned}
$$


Die Fouriertransformation von Slater-Funktionen durch rekursive Integration ist von McWeeny bereits im Jahr 1951 beschrieben worden [23] und Fouriertransformationen verschiedener Typen von Funktionen sind in der Literatur [24] angegeben. Zur Ableitung des Atomformfaktors des Wasserstoffatoms aus der Lösung der Schrödinger-Gleichung soll hier die geschlossene Form der Fourier-Bessel-Transformation verwendet werden, welche in der Literatur unter dem Stichwort „Closed-form expression for Fourier transform of Slatertype functions“ für Funktionen vom Typ der Gleichung 2.7 tabelliert wurde $[25,26,27]$. Häufig wird auch von einfachen $\zeta$-Slater-Funktionen (engl. single$\zeta$ Slater functions) gesprochen. In Gleichung 2.7 wird im Exponenten $Z$ statt $\zeta$ verwendet.

$$
\mathcal{G}_{N, k}(K, Z) \equiv \int_{0}^{\infty} r^{N} e^{-Z r} j_{k}(K r) d r
$$

$j_{k}(K r)$ ist die Besselfunktion mit $K=2 \pi r^{*}$. Durch Einsetzen von Gleichung 2.5 und dem Zusammenhang nach Bragg $\left(d=2 \cdot \frac{\sin \theta}{\lambda}\right)$ ergibt sich $r^{*}=2$. $\frac{a_{0} \sin \theta}{\kappa \lambda}$ und $K=4 \pi \frac{a_{0} \sin \theta}{\kappa \lambda}$. Die geschlossenen Fourier-Bessel-Transformation für $N=2$ und $k=0$ lautet:

$$
\mathcal{G}_{2,0}(K, Z) \equiv \frac{2 Z}{\left(K^{2}+Z^{2}\right)^{2}}
$$

Der Atomformfaktor des Wasserstoffatoms $f_{\mathrm{H}}\left(r^{*}\right)$ ist folglich

$$
\begin{gathered}
f_{\mathrm{H}}\left(r^{*}\right) \equiv 4 \cdot \int_{0}^{\infty} r^{2} e^{-2 r} j_{0}(K r) d r \\
f_{\mathrm{H}}\left(\frac{\sin \theta}{\lambda}\right) \equiv \frac{16}{\left[\left(4 \pi \frac{a_{0} \sin \theta}{\kappa \lambda}\right)^{2}+16\right]^{2}}
\end{gathered}
$$

Nach dem $\kappa$-Formalismus wurden in Gleichung 2.9 verschiedene $\kappa$-Werte verwendet. Die daraus resultierenden Atomformfaktoren des Wasserstoffatoms werden in Abbildung 2.3 gezeigt. Dabei ist der Atomformfaktor mit $\kappa=1,00$ die exakte Lösung der Schrödinger-Gleichung des freien Wasserstoffatoms. Im Modell unabhängiger Atome wird stattdessen jedoch der von Stewart et al. [28] berechneten Atomformfaktor des Wasserstoffmoleküls (SDS) verwendet, bei dem $\kappa=1,166$ beträgt. Das widerspricht zwar der Definition, stellt aber eine deutlich bessere Näherung an die experimentellen Beobachtungen dar. Für $\kappa=1,1$ und $\kappa=1,3$ sind ebenfalls Atomformfaktoren eingezeichnet. 


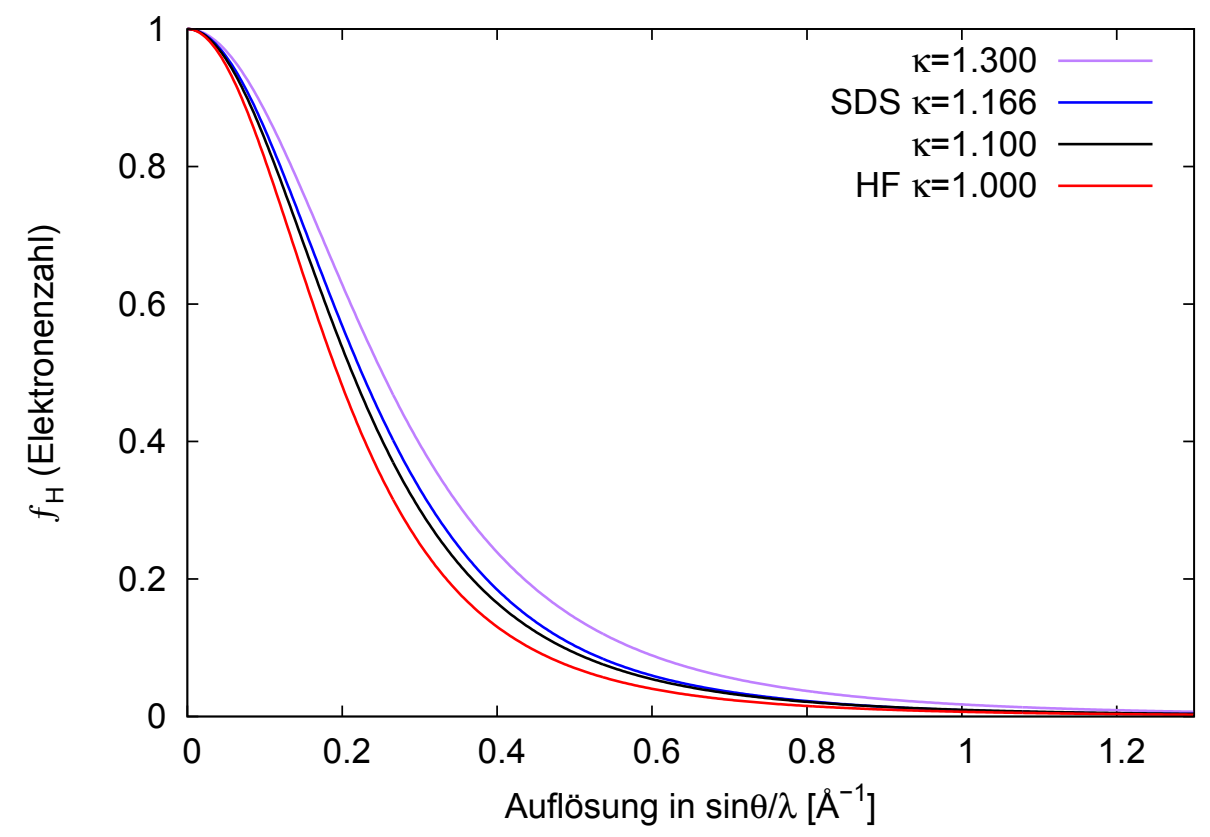

Abbildung 2.3: Der Atomformfaktor des Wassertoffatoms in Abhängigkeit des Kontraktionsparameters $\kappa$. IAM entspricht dem freien Wasserstoffatom (HF) mit $\kappa=1,0$. Für das Wasserstoffmolekül wurde nach Stewart et al. [28] $\kappa=1,166$ berechnet (SDS).

\subsection{Modell unabhängiger Atome}

Im Modell unabhängiger Atome (engl. Independent Atom Model, IAM), welches auch Promolekülmodell genannt wird, setzt sich die molekulare Elektronendichte aus kugelförmigen atomzentrierten Anteilen zusammen. Dass die Elektronendichte aufgrund der chemischen Bindungsumgebung asphärisch verzerrt ist, wird im IAM jedoch nicht berücksichtigt. Bei guten Kristallstrukturen mit $R(F)<5 \%$ kommt es daher häufig vor, dass Maxima der Restelektronendichte auf Bindungen und in Bereichen von freien Elektronenpaaren liegen, was die Schwächen des Modells deutlich macht. Durch Modifizierung der Modellannahmen kann eine bessere Übereinstimmung von Modell und Beobachtung erreicht werden. Trotz der relativ groben Näherung kugelförmiger Atome wird das IAM nach wie vor am häufigsten verwendet. Für Standardstrukturbestimmungen werden genauere asphärische Modelle nur selten verwendet. Die Wellenfunktionen schwererer Atome wurden von Clementi und Roetti für verschiedene Oxidationszustände in Form überlagerter Slater-Funktionen tabelliert [29]. Auch wenn die geschlossene Fourier-Bessel-Transformation die 
Ableitung der Atomformfaktoren einfach zugänglich macht, werden für den Atomformfaktor im IAM anstelle von Slater-Funktionen üblicherweise Gauß-Funktionen verwendet.

\subsection{1 Überlagerung von Gauß-Funktionen}

Die radialen Atomformfaktoren $f_{j}$ können gemäß Gleichung 2.10 durch Überlagerung von vier Gauß-Funktionen mit insgesamt neun Parametern interpoliert werden.

$$
f_{j}\left(\frac{\sin \theta}{\lambda}\right)=\sum_{i=1}^{4}\left[a_{i} \cdot e^{-b_{i}\left(\frac{\sin \theta}{\lambda}\right)^{2}}\right]+c
$$

Die Koeffizienten $a_{i}$, die Exponentialkoeffizienten $b_{i}$ und der Parameter zur Ordinatenkorrektur $c$ werden in Abhängigkeit vom Atomtyp variiert und sind in den „International Tables for Crystallography Volume C“ [30] zu finden. Für die Elemente Wasserstoff, Kohlenstoff, Stickstoff, Sauerstoff, Fluor, Chlor und das Chlorid-Anion, die in dieser Arbeit zur Verfeinerung von Strukturen verwendet werden, sind die Atomformfaktoren in Abbildung 2.4 dargestellt.

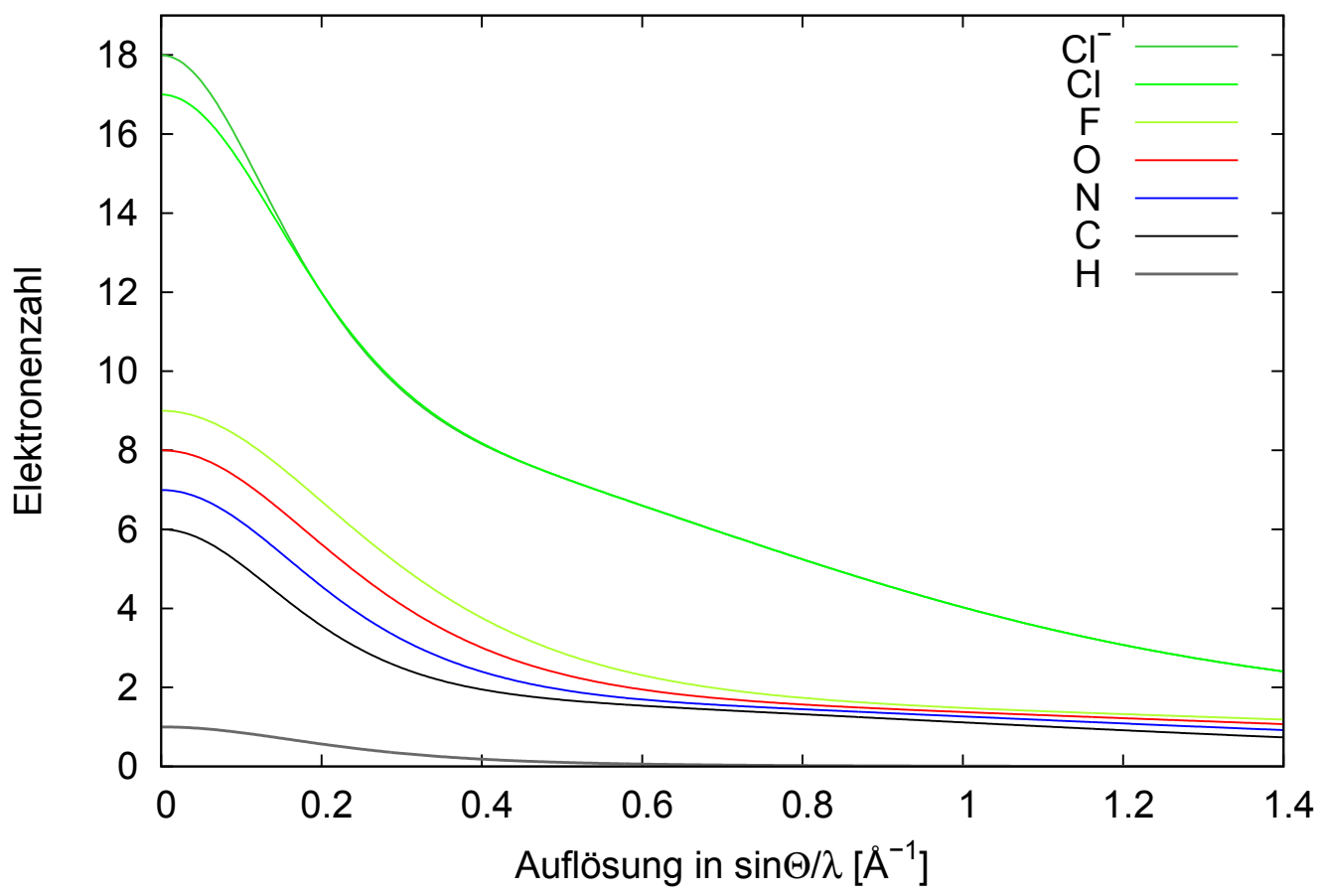

Abbildung 2.4: Die Atomformfaktoren von $\mathrm{H}, \mathrm{C}, \mathrm{N}, \mathrm{O}, \mathrm{F}, \mathrm{Cl}$ und $\mathrm{Cl}^{-}$ werden hier durch Überlagerung von vier Gauß-Funktionen interpoliert. 
Im Vergleich zu den schwereren Atomen ist die Streukraft von Wasserstoffatomen gering. Sie tragen ab einer Auflösung von $\frac{\sin \theta}{\lambda}>0,6 \AA^{-1}$ nicht mehr nennenswert zum Strukturfaktor bei. Die Atomformfaktoren des Chloratoms und des Chlorid-Anions sind nur im Auflösungsbereich zwischen $0,0 \AA^{-1}<$ $\frac{\sin \theta}{\lambda}<0,2 \AA^{-1}$ verschieden.

\subsubsection{Vergleich beider Formalismen von Atomformfaktoren}

In Abbildung 2.5 ist der Quotient der Atomformfaktorkurve des Wasserstoffatoms aus der Fouriertransformation der HF-Rechnung und der daran mit neun Parametern angenäherten Linearkombination von vier Gauß-Funktionen im Bereich von $0,0 \AA^{-1} \leq \frac{\sin \theta}{\lambda} \leq 1,3 \AA^{-1}$ gestrichelt eingezeichnet. Der Quotient liegt bis $0,6 \AA^{-1}$ bei „1“, was bedeutet, dass die Funktionen dort exakt übereinstimmen. Im Bereich von $0,6 \AA^{-1} \leq \frac{\sin \theta}{\lambda} \leq 1,3 \AA^{-1}$ gibt es leichte Schwankungen. In diesem Bereich ist der Beitrag zum Strukturfaktor allerdings insgesamt gering. Die Linearkombination der

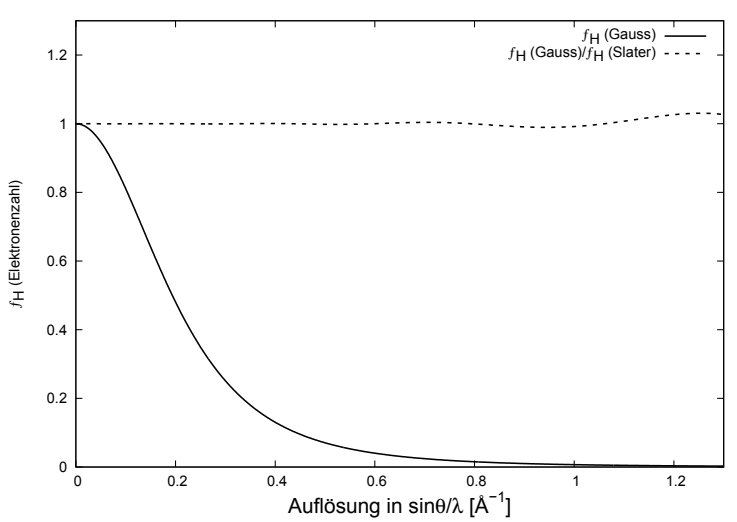

Abbildung 2.5: Vergleich der Atomformfaktorkurve des Wasserstoffatoms aus der Fouriertransformation der HF-Rechnung $f_{\mathrm{H}}($ Slater $)$ und der daran angenäherten Linearkombination von vier Gauß-Funktionen $f_{\mathrm{H}}(\mathrm{Gau} \beta)$. Der Quotient beider Funktionen ist gestrichelt aufgetragen. vier Gauß-Funktionen stellt also eine sehr gute Näherung an die fouriertransformierte Slater-Funktion dar.

\subsection{Ladungsdichtestudien mit dem Multipolmodell}

Für die Modellverbesserung ist die möglichst genaue Beschreibung der asphärischen Anteile der Elektronendichte in Form von Bindungselektronen und freien Elektronenpaaren entscheidend. Im häufig verwendetet Multipolmodell nach Hansen \& Coppens [31] ist die Beschreibung der Elektronendichte wie im Promolekülmodell atomzentriert. Die atomare Elektronendichte setzt sich aus einem Kern-, einem sphärischen Valenz- und einem asphärischen Valenzanteil 
zusammen.

$$
\rho_{\text {atom }}(\vec{r})=P_{\text {kern }} \rho_{\text {kern }}(\vec{r})+P_{\text {val }} \kappa^{3} \rho_{\text {val }}(\kappa \vec{r})+\sum_{l=0}^{l_{\text {max }}} \kappa^{\prime 3} R_{l}\left(\kappa^{\prime} \vec{r}\right) \cdot \sum_{m=-l}^{l} P_{l m} Y_{l m}(\vartheta, \varphi)
$$

Die Atomformfaktoren sind nicht mehr nur kugelförmig, sondern durch zusätzliche asphärische Anteile in der Lage Bindungselektronen und freie Elektronenpaare der Valenz abzubilden. Dies geschieht durch Besetzung von realen Kugelflächenfunktionen, wobei in der Verfeinerung bis Hexadecapol-Level pro Atom bis zu 25 zusätzliche Multipol-Parameter und zwei $\kappa$-Parameter $\left(\kappa, \kappa^{\prime}\right)$ eingeführt werden können. Nur bei subatomar aufgelösten Datensätzen mit $\mathrm{d} \leq 0,5 \AA \mathrm{bzw} \cdot \sin \theta / \lambda \geq 1,0 \AA^{-1}$ stehen dafür genug Reflexe zur Verfügung, um bei einem Reflex/Parameter-Verhältnis größer zehn zu bleiben. Das Multipolmodell wird auch als Pseudoatom-Modell bezeichnet.

\subsection{Transfer aus Datenbanken}

Asphärische Atomformfaktoren können auch aus den Datenbanken ELMAM (Experimental Library Multipolar Atom Model) [32], INVARIOM (Invariant Atoms) [33], UBDB (University of Buffalo Databank) [34] und SBFA (Supramolecular Synthon Based Fragments Approach) [35] transferiert werden. Alle Datenbanken enthalten bisher nur transferierbare Atomfragmente im Multipol-Formalismus. Die chemische Umgebung wird dabei näherungsweise berücksichtigt. Da die Anzahl der Parameter durch Verwendung asphärischer Atomformfaktoren aus Datenbanken nicht erhöht wird, sind sie für mittlere oder geringe Auflösung ebenso gut geeignet wie sphärische Atomformfaktoren. Die asphärischen Atomformfaktoren werden in der Literatur auch transferierbare atomaren Elektronendichtefragmente (engl. transferable atomic density fragment, TAD) genannt. Ein Vergleich der drei Pseudoatom-Datenbanken ELMAM, INVARIOM und UBDB in Bezug auf die Ableitung molekularer Eigenschaften wurde kürzlich vorgestellt [36]. Das aus dem Datenbank-Transfer stammende Modell der Elektronendichte wird dort als „transferierbares asphärisches Atom-Modell“ (engl. transferible aspherical atom model, TAAM) bezeichnet. Der automatisierte Transfer von Datenbankeinträgen auf ein Zielmolekül setzt voraus, dass die chemischen Umgebungen jedes Atoms eines Zielmoleküls erkannt und die entsprechenden Datenbankeinträge vorhanden sind. Zusätzlich ist beim Transfer der Datenbankeinträge die dreidimensionale Ausrichtung der Atomformfaktoren entscheidend. Um Transferierbarkeit unabhän- 
gig von Molekül oder Kristallsystem zu gewährleisten, besitzt jeder Atomformfaktor ein lokales, rechtwinkliges Koordinatensystem. Die Koordinatenachsen werden meist in Richtung der Nachbaratome ausgerichtet. In Datenbanken asphärischer Atomformfaktoren müssen deshalb sowohl die Elektronendichte als auch die Orientierung von Koordinatenachsen relativ zu Nachbaratomen gespeichert werden. Durch den Transfer asphärischer Atomformfaktoren auf ein Zielmolekül wird die molekulare Elektronendichte aus atomaren Elektronendichtefragmenten rekonstruiert. Der entscheidende Vorteil ist die genauere Beschreibung der molekularen Dichte, womit nicht nur eine bessere Übereinstimmung von Beobachtung und Modell erreicht werden kann, sondern auch die Berechnung molekularer Eigenschaften wie der elektrischen Momente oder des elektrostatischen Potentials unmittelbar aus der Röntgenstruktur zugänglich wird. Eine Übersicht und Literaturangaben befinden sich in Tabelle 2.1 am Ende des Kapitels.

\subsection{Quantenmechanische Modelle}

Zwei theoriebasierte Verfahren wurden von Jayatilaka und Mitarbeitern entwickelt und im Programm TONTO implementiert: Die Verfeinerung mit HirshfeldAtomen (engl. Hirshfeld-atom refinement, HAR) [37] und das Berechnen einer Wellenfunktion unter Einbeziehung experimenteller Röntgenbeugungsdaten (engl. wavefunction fitting) [38, 39]. Ähnlich wie bei experimentellen Elektronendichtebestimmungen mit dem Multipolmodell sollten für beide Verfahren Röntgenbeugungsdaten möglichst hoher Auflösung zur Verfügung stehen. Der entscheidende Vorteil liegt in der freien Wahl des Basissatzes und der quantenmechanischen Methode. Die Ergebnisse beider Verfahren können direkt mit denen quantenmechanischer Berechnungen verglichen werden und ermöglichen bei Wahl eines Basissatzes mit diffusen Funktionen auch die Berechnung der sog. Resonanzeigenschaften (engl. response properties). Dabei handelt es sich im Gegensatz zu den elektronischen Eigenschaften (engl. one-electron properties) um physikalische Größen, die nicht direkt aus der Elektronendichte zugänglich sind. Als Beispiele seien hier die Polarisierbarkeit oder Brechungsindizes genannt [40]. Für die Berechnung des ElektronenLokalisierungs-Index (ELI) zur Charakterisierung der Bindungsverhältnisse wird ebenfalls eine Wellenfunktion benötigt. Ein Nachteil der quantenmechanischen Verfahren besteht in der begrenzten Anwendbarkeit, da diese im Fall von Fehlordnung nicht durchgeführt werden können. Diese Einschränkung gilt auch für Strukturen, bei denen sich die Fehlordnung auf kokristallisierte Lösungs- 
mittelmoleküle beschränkt. Beide Verfahren sind zudem nicht für große Moleküle (z.B. Proteine) anwendbar, da sie sehr rechenintensiv sind.

\subsubsection{Verfeinerung mit Hirshfeld-Atomen}

Die atomaren Elektronendichtefunktionen werden hierbei direkt aus einer quantenmechanischen Single-Point-Energieberechnung entnommen. Um die in der Quantenmechanik üblichen Zwei-Zentren-Funktionale atomzentriert abzubilden wird die molekulare Dichte nach dem Anteilseignerprinzip atomzentriert unterteilt, welches 1977 von F. L. Hirshfeld [41] eingeführt wurde. Dafür wird eine Funktion $w(\vec{r})$ definiert, die den Anteil der sphärischen Dichten eines Atoms an der Gesamtelektronendichte im Kristall definiert.

$$
w(\vec{r})=\frac{\sum_{A_{A t o m}} \rho_{A}(\vec{r})}{\sum_{A_{\text {Krist }}} \rho_{A}(\vec{r})}
$$

Kristallographisch werden atomzentrierte Elektronendichtefunktionen $\rho_{j}(r)$ benötigt, da diese mit harmonischen Aufenthaltswahrscheinlichkeitsfunktionen $P_{j}(r)$, überlagert werden können, so dass die Auslenkung der Atome um ihre jeweilige Ruhelage in die Strukturfaktorberechnung einfließen kann.

$$
\left\langle\rho_{j}\right\rangle(r)=\rho_{j}(r) \star P_{j}(r) .
$$

Dabei ist $\left\langle\rho_{j}\right\rangle(r)$ das Hirshfeld-Atom, das zur Strukturfaktorberechnung verwendet wird und $\star$ repräsentiert die Faltung.

Im Anschluss werden die Koordinaten und Auslenkungsparameter mit der Methode der kleinsten Quadrate verfeinert. Die berechneten Hirshfeld-Atome sind nach Debye (1930) näherungsweise rigide. Allerdings werden die atomaren Koordinaten durch die Verfeinerung modifiziert. Da sowohl die molekulare Dichte als auch die Hirshfeldsche Gewichtungsfunktion unmittelbar davon abhängig sind, wird die Berechnung von Hirshfeld-Atomen mit anschließender Verfeinerung üblicherweise bis zur Konvergenz iterativ wiederholt.

Die Verfeinerung mit Hirshfeld-Atomen ist mit einem großen numerischen Aufwand verbunden. Dafür werden weniger Parameter als bei der experimentellen Elektronendichtebestimmung einer Multipol-Verfeinerung benötigt.

Um die Anwendbarkeit auf größere Moleküle auszudehnen, ist die Verwendung von Hirshfeld-Atomen für den Einsatz in der Invariom-Datenbank geplant. Der 
entscheidende Vorteile gegenüber dem Multipolmodell nach Hansen \& Coppens liegt in der freien Wahl von Basissatz und Methode. Zusätzlich können Kristalleffekte näherungsweise in die Berechnung einbezogen werden, indem ab dem zweiten Iterationsschritt Punktladungen aus den Hirshfeld-Atomen berechnet werden, welche entsprechend der kristallographischen Symmetrie als Cluster um die asymmetrische Einheit platziert werden. Dadurch wird eine so hohe Genauigkeit der Atomformfaktoren erreicht, dass anisotrope Auslenkungsparameter von Wasserstoffatomen verfeinert werden können [37], welche ansonsten nur in Neutronenbeugungsexperimenten beobachtet werden können [42].

\subsubsection{Annäherung einer Wellenfunktion an experimentelle Daten}

Die Einbeziehung experimenteller Daten bei der Berechnung von Wellenfunktionen erfolgt im Programm TONTO über den Lagrange-Multiplikator $\lambda$ bei gleichzeitiger Energieminimierung [38, 39]. Für die Anpassung der Wellenfunktion an die experimentellen Daten kann $\lambda$ als Gewichtungsfaktor zwischen den experimentellen Daten und der Energieminimierung bezeichnet werden.

$$
L(\mathbf{c}, \epsilon, \lambda)=E(\mathbf{c}, \epsilon)-\lambda\left[\chi^{2}(\mathbf{c})-\Delta\right]
$$

Die Energie hängt von den Koeffizienten c der verwendeten Wellenfunktion ab. Die Übereinstimmung der Wellenfunktion mit den experimentellen Daten wird dabei über die $\chi^{2}$-Statistik in die Lagrange-Funktion einbezogen. Die Strukturfaktoren $F_{c}(\vec{h})$ werden hier durch Fouriertransformation der Wellenfunktion berechnet.

$$
\chi^{2}=\sum_{\vec{h}}^{N_{r}} \frac{w(\vec{h})\left[F_{o}(\vec{h})-F_{c}(\vec{h})\right]^{2}}{n_{\mathrm{ref}}-n_{\mathrm{par}}}
$$

Die $\chi^{2}$ Statistik wird auch bei der Verfeinerung mit Hirshfeld-Atomen verwendet und entspricht bis darauf, dass hier die Strukturfaktoren $F_{o}(\vec{h})$ anstelle der Strukturfaktorquadrate $F_{o}^{2}(\vec{h})$ verwendet werden, dem Quadrat der Anpassungsgüte $S$ (Goof) welche in Gleichung 1.18 bei der konventionellen Verfeinerung von Röntgenbeugungsdaten eingeführt wurde. Zur Berechnung von Strukturfaktoren aus der Wellenfunktion der berechneten molekularen Einheit $\Psi_{\text {Einheit }}$ wird näherungsweise angenommen, dass diese nicht mit benachbarten Einheiten wechselwirkt. Dadurch kann $\Psi_{\text {Einheit }}$ näherungsweise isoliert be- 
handelt und über die im Kristall vorhandenen Symmetrieoperationen kopiert werden, um die Elektronendichte der Elementarzelle zu rekonstruieren.

\subsection{Vergleich der Modelle}

In Tabelle 2.1 sind alle zuvor beschriebenen Elektronendichte-Modelle zusammen gefasst. $\mathrm{Zu}$ jedem Modell sind auch die zur Erzeugung der atomaren Streufaktoren $f_{j}$ verwendeten Methoden, die heute gängigsten Programme, mögliche zu verfeinernde Parameter und Literaturangaben aufgeführt. 


\begin{tabular}{|c|c|c|c|c|c|c|c|c|c|c|}
\hline & 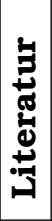 & 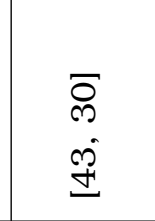 & 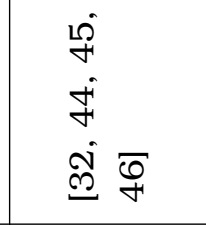 & $\begin{array}{l}\dot{m} \\
\dot{+} \\
\dot{+} \\
\dot{\dot{\theta}}\end{array}$ & 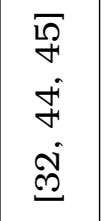 & $\begin{array}{l}\bar{\sigma} \\
\infty \\
\stackrel{\infty}{0}\end{array}$ & $\begin{array}{l}\overline{\overrightarrow{0}} \\
\dot{0} \\
\dot{0} \\
\dot{0}\end{array}$ & $\begin{array}{l}\bar{D} \\
\stackrel{\infty}{0}\end{array}$ & $\begin{array}{l}\hat{\infty} \\
\text { ô } \\
\text { bै }\end{array}$ & $\begin{array}{l}\bar{N} \\
\text { ஸे } \\
\dot{0} \\
\infty \\
\infty \\
0\end{array}$ \\
\hline 8 & 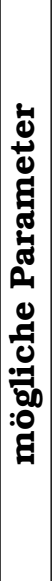 & 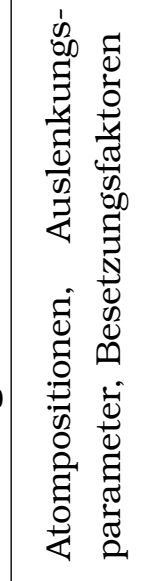 & 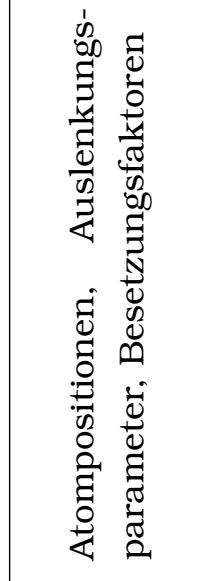 & 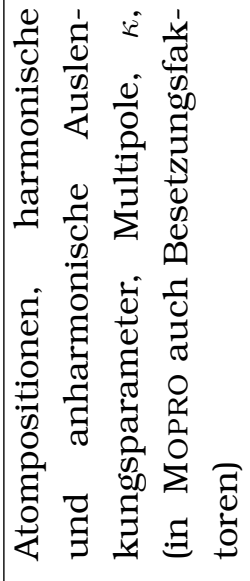 & \multicolumn{4}{|c|}{ 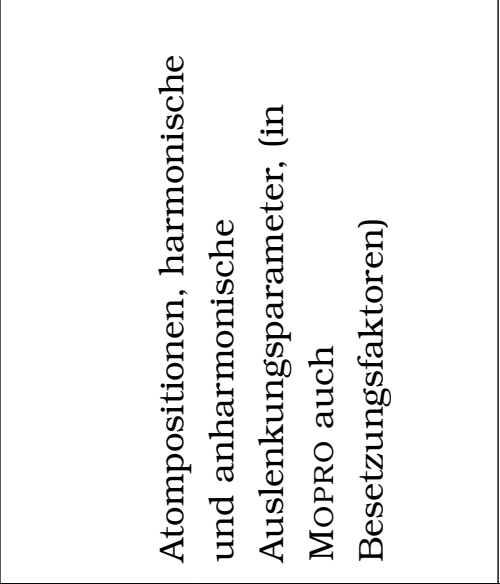 } & 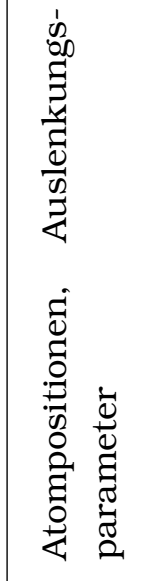 & 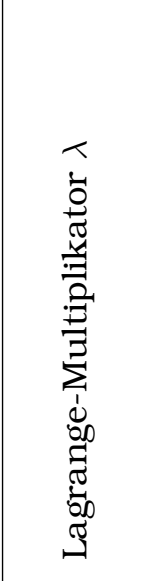 \\
\hline & & 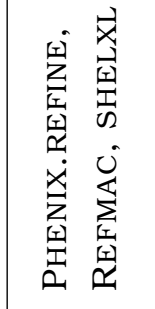 & $\begin{array}{l}00 \\
\dot{x} \\
\dot{\alpha} \\
\frac{\alpha}{0} \\
0 \\
\sum\end{array}$ & 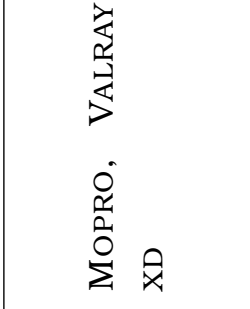 & $\begin{array}{l}0 \\
\text { qu } \\
\text { O } \\
\Sigma\end{array}$ & $\begin{array}{l}\hat{x} \\
0 \\
0 \\
0 \\
0 \\
\vdots \\
\Sigma\end{array}$ & $\ddot{x}$ & $x$ & $\begin{array}{l}0 \\
\stackrel{\circ}{z} \\
\stackrel{0}{\Theta} \\
\Leftrightarrow\end{array}$ & 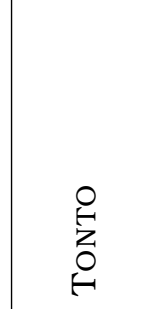 \\
\hline \multirow{4}{*}{ 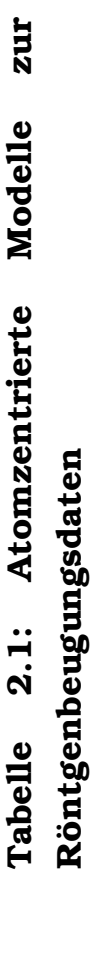 } & 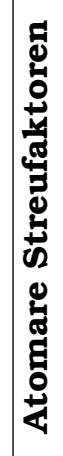 & 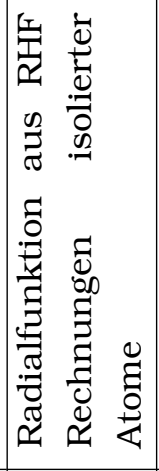 & 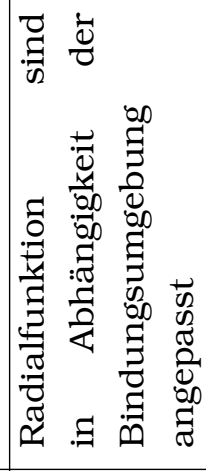 & 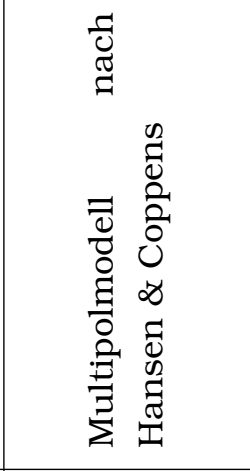 & 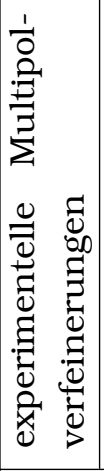 & 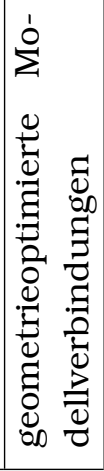 & 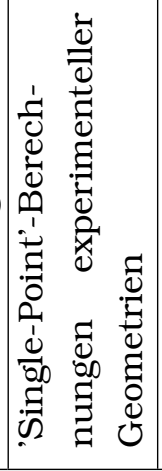 & 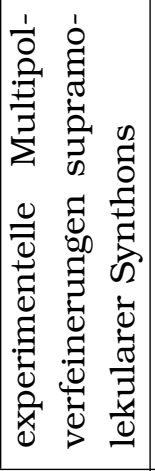 & 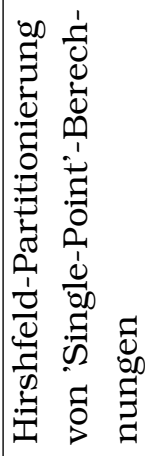 & 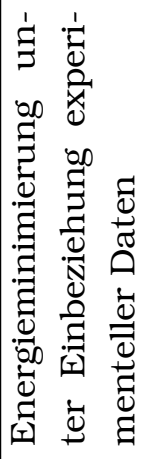 \\
\hline & & & & & \multicolumn{4}{|c|}{ 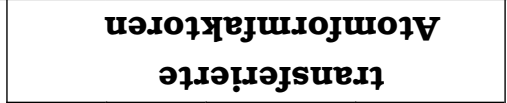 } & \multirow[b]{2}{*}{ 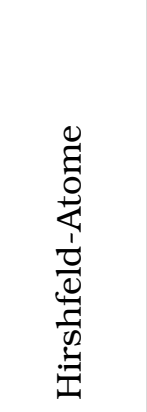 } & \multirow[b]{2}{*}{ 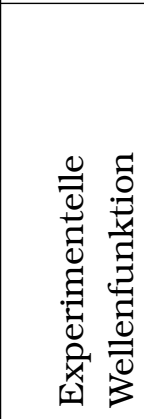 } \\
\hline & $\begin{array}{l}0 \\
\mathbb{Z} \\
\mathbb{E} \\
\mathbf{Z}\end{array}$ & 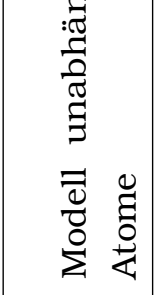 & 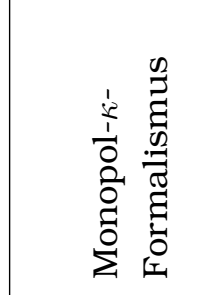 & 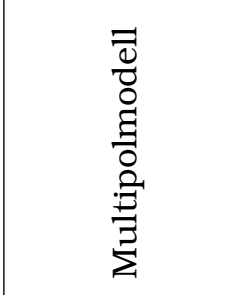 & 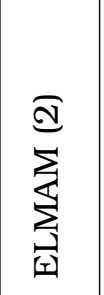 & 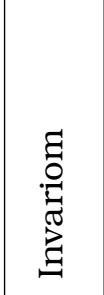 & $\begin{array}{l}\stackrel{0}{\circ} \\
\stackrel{\varphi}{b}\end{array}$ & $\begin{array}{l}\overleftrightarrow{\mid} \\
\text { 品 }\end{array}$ & & \\
\hline & & \multicolumn{2}{|c|}{ पos!..rẹपds } & \multicolumn{7}{|c|}{ पos!ుẹप } \\
\hline
\end{tabular}




\section{Kapitel 3}

\section{Molekulare Eigenschaften}

\subsection{Topologische Analyse der Elektronendichte}

Während Moleküle quantenmechanisch durch die Wellenfunktion beschrieben werden, verwenden Chemiker gern das Konzept von Atomen und funktionellen Gruppen, aus denen sich die Moleküle zusammensetzen. Die physikalische Grundlage dafür liefert die „Quantentheorie von Atome in Molekülen“ (QTAIM) von Richard F. W. Bader [53]. Dabei handelt es sich um ein Partitionierungsschema, mit dem es durch topologische Analyse der Elektronendichteverteilung möglich ist, Moleküle in atomare Fragmente (oder funktionelle Gruppen) zu zerlegen. Dadurch können Eigenschaften wie atomare Volumina und Ladungen sowie die Bindungsstärke quantitativ erfasst werden [54, 55]. Die Elektronendichte ist im Gegensatz zur Wellenfunktion eine Observable des Röntgenbeugungsexperiments und kann als kontinuierliches Skalarfeld im dreidimensionalen Raum aufgefasst werden.

\subsection{Kritische Punkte}

Für die topologische Analyse der Elektronendichte werden die ersten und die zweiten partiellen Ortsableitungen gebildet. Die ersten Ortsableitungen stellen das Gradientenvektorfeld dar. Ein Extremum in der ersten Ableitung wird kritischer Punkt (KP) genannt und liegt vor, wenn die Bedingung

$$
\nabla \rho\left(\vec{r}_{\mathrm{KP}}\right)=\left(\begin{array}{c}
\frac{\partial \rho}{\partial x} \\
\frac{\partial \rho}{\partial y} \\
\frac{\partial \rho}{\partial z}
\end{array}\right)=0
$$


erfüllt ist. Da es Extrema am Ort des Kerns, auf Bindungen, in Ringen und in Käfigen gibt, ist eine weitere Klassifizierung nach mathematischen Gesichtspunkten notwendig. Die Hesse-Matrix $H(\vec{r})$ beinhaltet alle zweiten partiellen Ortsableitungen der Elektronendichte:

$$
H(\vec{r})=\left(\begin{array}{ccc}
\frac{\partial^{2} \rho}{\partial x^{2}} & \frac{\partial^{2} \rho}{\partial x \partial y} & \frac{\partial^{2} \rho}{\partial x \partial z} \\
\frac{\partial^{2} \rho}{\partial y \partial x} & \frac{\partial^{2} \rho}{\partial y^{2}} & \frac{\partial^{2} \rho}{\partial y \partial z} \\
\frac{\partial^{2} \rho}{\partial z \partial x} & \frac{\partial^{2} \rho}{\partial z \partial y} & \frac{\partial^{2} \rho}{\partial z^{2}}
\end{array}\right)
$$

Die diagonalisierte Hesse-Matrix $D(\vec{r})$ enthält nur noch die Diagonalelemente, die mit $\lambda_{n}$ bezeichnet werden:

$$
D(\vec{r})=\left(\begin{array}{ccc}
\frac{\partial^{2} \rho}{\partial x^{2}} & 0 & 0 \\
0 & \frac{\partial^{2} \rho}{\partial y^{2}} & 0 \\
0 & 0 & \frac{\partial^{2} \rho}{\partial z^{2}}
\end{array}\right)=\left(\begin{array}{ccc}
\lambda_{1} & 0 & 0 \\
0 & \lambda_{2} & 0 \\
0 & 0 & \lambda_{3}
\end{array}\right)
$$

Ist die zweite Ortsableitung entlang einer Raumrichtung negativ, so handelt es sich um ein Maximum in dieser Richtung, ist sie positiv, um ein Minimum. Die Klassifizierung der kritischen Punkte der Elektronendichte erfolgt mit einem Paar von Krümmungsparametern $(\omega, \sigma)$, das sich aus den Diagonalelementen der Hesse-Matrix $D(\vec{r})$ erschließt. $\omega$ ist die Anzahl und $\sigma$ die Vorzeichensumme der Extrema von $\lambda_{1}, \lambda_{2}$ und $\lambda_{3}$. Bei einem kernkritischen Punkt handelt es sich um ein dreifaches lokales Maximum der Elektronendichte $(3,-3)$. Ein bindungskritischer Punkt (BKP) ist ein Sattelpunkt, der entlang des Bindungpfades ein Minimum und jeweils senkrecht dazu ein Maximum in $\rho(\vec{r})$ besitzt $(3,-1)$. Es ist also der Punkt entlang einer Bindung mit der geringsten Elektronendichte. Ein ringkritischer Punkt (RKP) ist auch ein Sattelpunkt, der allerdings ein doppeltes Minimum von $\rho(\vec{r})$ entlang der Ringebene und ein Maximum senkrecht dazu aufweist $(3,+1)$. Der käfigkritische Punkt (KKP) besitzt ein dreifaches lokales Minimum in $\rho(\vec{r})(3,+3)$.

Tabelle 3.1: Klassifizierung der kritischen Punkte nach ihren Krümmungsparametern

\begin{tabular}{|l|ccc|c|}
\hline Art des kritischen Punktes & $\lambda_{1}$ & $\lambda_{2}$ & $\lambda_{3}$ & $(\omega, \sigma)$ \\
\hline Kernkritischer Punkt & - & - & - & $(3,-3)$ \\
Bindungskritischer Punkt & - & - & + & $(3,-1)$ \\
Ringkritischer Punkt & - & + & + & $(3,+1)$ \\
Käfigkritischer Punkt & + & + & + & $(3,+3)$ \\
\hline
\end{tabular}


Mit der Analyse der bindungskritischen Punkte können Bindungseigenschaften untersucht werden. Dabei werden die Werte der Elektronendichte, der Laplacefunktion und der Bindungselliptizität am bindungskritischen Punkt interpretiert.

Mit der Elliptizität wird die Abweichung von einer zylindersymmetrischen Elektronendichteverteilung um die Bindungsachse quantifiziert. Die Bindungselliptizität $\epsilon$ ist in 3.4 definiert als das Verhältnis der beiden negativen zweiten Ortsableitungen des bindungskritischen Punktes.

$$
\epsilon=\frac{\lambda_{1}}{\lambda_{2}}-1
$$

\subsection{Laplacefunktion}

Die Laplacefunktion wird durch Anwendung des Laplaceoperators auf die Elektronendichte erhalten und ist gleichzeitig die Spur der diagonalisierten HesseMatrix.

$$
\nabla^{2} \rho(\vec{r})=\frac{\partial^{2} \rho(\vec{r})}{\partial x^{2}}+\frac{\partial^{2} \rho(\vec{r})}{\partial y^{2}}+\frac{\partial^{2} \rho(\vec{r})}{\partial z^{2}}=\lambda_{1}+\lambda_{2}+\lambda_{3}
$$

Die Laplacefunktion liefert Informationen über die Ladungsverteilung. An Punkten (starker) lokaler Ladungshäufung ist die Laplacefunktion negativ und an Punkten (starker) lokaler Ladungsverringerung positiv. Dadurch können freie Elektronenpaare als lokale Minima der Laplacedichte gefunden werden. Sie erlaubt also die Lokalisierung der Valenzschalenladungskonzentration (engl. valence shell charge concentration VSCC). Die Null-Laplace-Oberfläche bezeichnet die Isooberfläche der Laplacedichte bei dem Wert null. Regionen, in denen die Laplacedichte den Wert null nicht annimmt, sind Orte verringerter Valenzschalenladungskonzentration. Anhand von Löchern in der Null-LaplaceOberfläche können elektrophile Zentren des Moleküls gefunden werden, die bevorzugt von Nukleophilen angegriffen werden.

\subsection{Atomare Bassins}

Durch den Verlauf der Trajektorien des Gradientenvektorfeldes ist die Partitionierung eines Moleküls in seine atomaren Bestandteile möglich. Da die Gradienten im Kern, dem sog. Attraktor enden und niemals die sog. flusslosen Flächen (engl. zero flux surfaces, ZFS) durchqueren, können alle Gradienten 
einem Kern zugeordnet werden. Die flusslosen Flächen bilden die Grenzflächen zwischen den Atomen und begrenzen damit die atomaren Bassins $\Omega$. Für die flusslosen Flächen gilt: Der Normalenvektor $\vec{n}$ der flusslosen Fläche steht senkrecht auf dem Gradienten der Elektronendichte an dieser Stelle.

$$
\nabla \rho(\vec{r}) \cdot \vec{n}=0
$$

Durch die Zuteilung eines diskreten Raumbereichs zu einem Atom wird die Bestimmung atomarer Eigenschaften möglich. Das Volumen des Atoms ergibt sich aus der Integration über alle Volumenelemente $d \tau$ des Bassins:

$$
V(\Omega)=\int_{\Omega} d \tau
$$

Des Weiteren kann die Elektronenzahl durch Integration der Elektronendichte über die Volumenelemente des Bassins erhalten werden:

$$
N(\Omega)=\int_{\Omega} \rho(\vec{r}) d \tau
$$

Die Differenz aus Kernladungszahl $Z$ und der Elektronenzahl des atomaren Bassins $N(\Omega)$ ergibt die atomare Ladung $Q(\Omega)$ :

$$
Q(\Omega)=Z-N(\Omega)
$$

\subsection{Hirshfeld-Oberflächen}

Hirshfeld-Oberflächen beruhen auf einem Partitionierungsschema der Elektronendichte im Kristall, das sich auf Hirshfelds Anteilseignerprinzip [41] bezieht. Die Wichtungsfunktion $w(\vec{r})$ ist definiert als der Guotient aus der Summe der sphärischen Dichten der Atome des Moleküls und der Summe der sphärischen Dichten der Atome des Kristalls [56]:

$$
w(\vec{r})=\frac{\sum_{A_{M o l}} \rho_{A}(\vec{r})}{\sum_{A_{K r i s t}} \rho_{A}(\vec{r})}
$$

Die Hirshfeld-Oberfläche ist die Begrenzung des Moleküls, an der $w(\vec{r})=0,5$ gilt. Der Raumanspruch eines Moleküls im Kristall wird definiert durch die Re- 
gion, in der gilt $w(\vec{r}) \geq 0,5$. Innerhalb der Hirshfeld-Oberfläche ist der Anteil, den das Molekül zur Dichte im Kristall beiträgt größer als die Summe der Anteile der benachbarten Moleküle. Nach dieser Definition sind im Kristall auch Regionen vorhanden, die keinem Molekül zugeordnet werden können, so dass die Raumausfüllung geringer als $100 \%$ ist.

\subsection{Elektrostatisches Potential}

Beim elektrostatischen Potential $V(\vec{r})$ handelt es sich um eine Energiegröße, die den Aufwand angibt, eine positive Punktladung aus dem Unendlichen an einen Ort $\vec{r}$ zu bringen. Es ist also ein Maß für die potentielle Energie einer positiven Punktladung an einem bestimmten Ort im Raum. Regionen eines Moleküls mit negativem elektrostatischem Potential (ESP), können leicht von elektrophilen Teilchen angegriffen werden. Umgekehrt fungieren Bereiche des Moleküls mit positivem ESP selbst als Elektrophile. Die Reaktivität eines Moleküls lässt sich mit dem ESP quantitativ bestimmen. Es wird nach $\mathrm{Su}$ und Coppens [57] gemäß Gleichung 3.11 direkt aus der Elektronendichte berechnet. Dabei wird über alle $n$ Kerne des Moleküls mit ihren jeweiligen Kernladungen $Z_{n}$ und Kernpositionen $\vec{R}_{n}$ summiert und davon der Beitrag der Elektronen anhand der Dichte im Umkreis $d \vec{r}^{\prime}$ um den Ort $\vec{r}$ abgezogen.

$$
V(\vec{r})=\sum_{n} \frac{Z_{n}}{\left|\vec{R}_{n}-\vec{r}\right|}-\int \frac{\rho\left(\vec{r}^{\prime}\right)}{\left|\vec{r}-\vec{r}^{\prime}\right|} d \vec{r}^{\prime}
$$

Die Implementierung der Berechnung des elektrostatischen Potentials im Programm XDPROP des XD Programmpakets [46] wurde von Volkov et. al [58] durch Anwendung der EP/MM-Näherung (engl. Exact Potential/Multipole Method) nach Buckingham [59] verbessert. Es hat sich als hilfreich herausgestellt das elektrostatische Potential auf eine molekulare Oberfläche zu kartieren, um elektrostatische Wechselwirkungen zwischen Molekülen zu untersuchen. Dafür kann die Hirshfeld-Oberfläche [60, 61, 62] oder eine Isooberfläche der Elektronendichte verwendet werden. Um das elektrostatische Potential im Van-derWaals-Abstand um ein Molekül zu erhalten wird eine Isooberfläche bei einer Elektronendichte von 0,001 au $\left(0,0067 \mathbf{e} / \AA^{3}\right)$ verwendet. 


\subsubsection{Politzer-Analyse}

Das ESP lässt sich auch quantitativ analysieren. Hierzu wird der Formalismus von Politzer et al. [63] verwendet. Das positive, negative und gesamte durchschnittliche Potential auf der Oberfläche $\left(\bar{V}_{S}^{+}, \bar{V}_{S}^{-}\right.$und $\left.\bar{V}_{S}\right)$ wird gemäß der Gleichungen 3.12 bis 3.14 berechnet, wobei $n$ und $m$ die Anzahl der Oberflächenpunkte mit positivem beziehungsweise negativem Potential des Gitters sind.

$$
\begin{gathered}
\bar{V}_{S}^{+}=\frac{1}{n} \sum_{i=1}^{n} V_{S}^{+}\left(\vec{r}_{i}\right) \\
\bar{V}_{S}^{-}=\frac{1}{m} \sum_{i=1}^{m} V_{S}^{-}\left(\vec{r}_{i}\right) \\
\bar{V}_{S}=\frac{1}{m+n}\left[\sum_{i=1}^{n} V_{S}^{+}\left(\vec{r}_{i}\right)+\sum_{i=1}^{m} V_{S}^{-}\left(\vec{r}_{i}\right)\right]
\end{gathered}
$$

Die durchschnittliche Abweichung vom Mittelwert des Oberflächenpotentials $\Pi$ ist gegeben durch:

$$
\Pi=\frac{1}{m+n} \sum_{i=1}^{m+n}\left|V_{S}\left(\vec{r}_{i}\right)-\bar{V}_{S}\right|
$$

Die positive, negative und totale Varianz der Oberflächenpotentiale wird gemä $\beta$ 3.16 berechnet.

$$
\sigma_{t o t}^{2}=\sigma_{+}^{2}+\sigma_{-}^{2}=\frac{1}{n} \sum_{i=1}^{n}\left[V_{S}^{+}\left(\vec{r}_{i}\right)-\bar{V}_{S}^{+}\right]^{2}+\frac{1}{m} \sum_{i=1}^{m}\left[V_{S}^{-}\left(\vec{r}_{i}\right)-\bar{V}_{S}^{-}\right]^{2}
$$

Die quantitative Analyse des auf eine Oberfläche kartierten elektrostatischen Potentials ist im Programm MOLISo [64] implementiert und wurde in der Literatur bereits in den Ladungsdichtestudien des Nukleosids Thymidine [65], des Makrolid-Antibiotikums Roxithromycin [66] und der vergleichenden Studie der asphärischen Atomformfaktor-Datenbanken [36] angewendet.

\subsection{Stärke von Wasserstoffbrückenbindungen}

Aus der Elektronendichte $\rho\left(\vec{r}_{\mathrm{BKP}}\right)$ und der Laplacefunktion $\nabla^{2} \rho\left(\vec{r}_{\mathrm{BKP}}\right)$ am bindungskritischen Punkt wurde ein Zusammenhang mit der kinetischen Ener- 
giedichte $G\left(\vec{r}_{\mathrm{BKP}}\right)$, der potentiellen Energiedichte $V\left(\vec{r}_{\mathrm{BKP}}\right)$ und der Wasserstoffbrückenbindungsenergie $E_{\mathrm{HB}}$ empirisch hergeleitet. Die kinetische Energiedichte $G\left(\vec{r}_{\mathrm{BKP}}\right)$ kann nach Abramov et al. [67] gemäß Gleichung 3.17, die potentielle Energiedichte $V\left(\vec{r}_{\mathrm{BKP}}\right)$ und die Wasserstoffbrückenbindungsenergie $E_{\mathrm{HB}}$ nach Espinosa et. al [68] gemäß der Gleichungen 3.18 und 3.19 berechnet werden.

$$
\begin{gathered}
G\left(\vec{r}_{\mathrm{BKP}}\right)=\frac{3(3 \pi)^{\frac{2}{3}}}{10} \rho\left(\vec{r}_{\mathrm{BKP}}\right)^{\frac{5}{3}}+\frac{\nabla^{2} \rho\left(\vec{r}_{\mathrm{BKP}}\right)}{6} \\
V\left(\vec{r}_{\mathrm{BKP}}\right)=-\frac{3(3 \pi)^{\frac{2}{3}}}{5} \rho\left(\vec{r}_{\mathrm{BKP}}\right)^{\frac{5}{3}}-\frac{\nabla^{2} \rho\left(\vec{r}_{\mathrm{BKP}}\right)}{12} \\
E_{\mathrm{HB}}=\frac{1}{2} \cdot V\left(\vec{r}_{\mathrm{BKP}}\right)
\end{gathered}
$$




\section{Teil II}

\section{Methoden und Experimente}





\section{Kapitel 4}

\section{Messplätze}

Die in dieser Arbeit ausgewerteten Datensätze wurden an drei SynchrotronMessplätzen und drei verschiedenen Diffraktometern innerhalb des Instituts gemessen. Der jeweils verwendete Messplatz wurde in Abhängigkeit der Kristallgröße ausgewählt.

\subsection{Australian Synchrotron}

Der Messplatz für Protein-Kristallographie am Australian Synchrotron verfügt über ein Bruker-Diffraktometer mit $\kappa$-Geometrie und ist mit einem MAR 345 Detektor (Image-Plate) und einer offenen Stickstoffkaltgasstromkühlung der Firma Oxford Cryosystems ausgestattet.

\subsection{Deutsches Elektronen-Synchrotron (DESY)}

Der Messplatz an Beamline F1 des HASYLAB, DESY in Hamburg, Deutschland verfügt über ein Huber-Diffraktometer mit $\kappa$-Geometrie und ist mit einer offenen Stickstoffkaltgasstromkühlung der Firma Oxford Cryosystems und einem ein MarCCD 165 Flächendetektor ausgestattet.

\subsection{Swiss Light Source (SLS)}

Der Messplatz X10SA (PXII) am SLS, des Paul-Scherrer-Instituts in Villigen, Schweiz ist mit einem MarCCD 225 Flächendetektor ausgestattet und verfügt 
über eine Stickstoffkaltgasstromkühlung. Als Synchrotron dritter Generation verfügt es über besonders hohe Primärstrahlintensität.

\subsection{Messungen an der Universität Göttingen}

Die verwendeten Diffraktometer am Institut für Anorganische Chemie der Arbeitsgruppe von Prof. Stalke sind jeweils mit einem Dreikreisdiffraktometer und einem APEX II CCD-Flächendetektor sowie einer offenen Stickstoffkaltgasstromkühlung der Firma Bruker ausgestattet. Als Strahlenquellen sind eine $\operatorname{MoK} \alpha$-Microsource und eine $\operatorname{AgK} \alpha$-Microsource der Firma Incoatec installiert.

Das Dreikreisdiffraktometer der Arbeitsgruppe von Prof. Sheldrick ist mit einem SMART 6000-Flächendetektor und einer offenen Stickstoffkaltgasstromkühlung der Firma Oxford Cryosystems ausgestattet. Als Stahlenquelle steht eine $\mathrm{CuK} \alpha$-Drehanode der Firma MacScience mit einer Helios Spiegeloptik von Incoatec zur Verfügung. 


\section{Kapitel 5}

\section{Invariome}

Der Begriff Invariom ist eine Wortneuschöpfung, die sich aus dem Ausdruck invariantes Atom ableitet. Den Invariomen liegt die Beobachtung zu Grunde, dass nur die Atome einer Elementsorte invariant sind, deren chemische Umgebung näherungsweise gleich ist. Allgemein gesprochen ist ein Invariom ein starres, atomares Elektronendichtefragment [69]. Dabei wird die atomare Bindungsumgebung durch den Invariom-Namen charakterisiert. Neben der Art der gebundenen Elemente spielt auch die Bindungsordnung (BO) eine wichtige Rolle. Invariome werden vor allem als aspährische Atomformfaktoren in der Röntgenstrukturanalyse verwendet. Zur Unterscheidung und Zuordnung von Invariomen an Atome ist es sinnvoll eine Notation für Invariome einzuführen, welche in Abschnitt 5.2 vorgestellt wird. Da durch die chemische Umgebung eines Atoms oft nur ein Molekülfragment definiert ist, unterscheidet sich der Invariom-Name von anderen linearen Notationen, wie IUPAC, SMILES oder Trivialnamen, welche für ein ganzes Molekül stehen. Ein Invariom kann also in verschiedenen Molekülen enthalten sein und ist zwischen diesen übertragbar. Die Transferierbarkeit von Invariomen ähnelt dem Chemikern geläufigen Prinzip funktioneller Gruppen. Hierbei wird für eine funktionelle Gruppe in verschiedenen Molekülen die gleiche (oder sehr ähnliche) Reaktivität angenommen.

\subsection{Konzept der Bindungsordnung}

In der bisherigen Konvention der Invariome [70] wurde der Bindungsunterscheidungsparameter $\chi$ zur Ermittlung der Bindungsordnung verwendet. Bei der Berechnung des Bindungsunterscheidungsparameters werden gemäß Glei- 
chung 5.1 die Kovalenzradien und die Elektronegativitätsdifferenz nach Allred und Rochow [71] für die an der Bindung beteiligten Atome einbezogen.

$$
\chi=\left[r_{K}(\text { Atom } 1)+r_{K}(\text { Atom } 2)-0.08 \cdot|\Delta(E N)|\right]-\mathrm{d}
$$

Für den Bindungsunterscheidungsparameter gibt es vier Wertebereiche. Liegt der $\chi$-Wert unterhalb von 0,0847 handelt es sich um eine Einfachbindung $(\mathrm{BO}=1)$. Eine Bindung mit dem Bindungsgrad $\mathrm{BO}=1.5$ verfügt über $\pi$-Bindungsanteil der z.B. durch Mesomeriestabilisierung bedingt ist. Der $\chi$-Wert liegt dafür zwischen 0,0847 und 0,1840. Ein $\chi$-Wert im Bereich von 0,1840 und 0,27 zeigt eine Doppelbindung $(\mathrm{BO}=2)$ an. Oberhalb von 0,27 liegt eine Dreifachbindung $(\mathrm{BO}=3)$ vor. Dieses Vorgehen hat eine gewisse Eleganz, wenn man bedenkt, dass durch den Bindungsunterscheidungsparameter $\chi$ die Bindungsumgebungen aller Aminosäuren und ihrer Peptide charakterisiert [33] und automatisiert zugeordnet werden konnten [49]. Bei komplexeren (z.B heteroaromatischen) Molekülen können jedoch Uneindeutigkeiten bei der Zuordnung der Bindungsordnung mit dem $\chi$-Parameter auftreten, weshalb die geometrische Koordination der Atome als zusätzliches Kriterium bei der Bestimmung der Bindungsordnung einbezogen wurde. Die Bindungsordnung zu benachbarten Atomen, die auch Teil eines aromatischen (oder dreigliedrigen) Rings sind, wird durch die Kombination des Symbols „\#“ und der Ringgröße (Anzahl der Atome im Ring) angegeben. Auch die Bindungsordnung von Substituenten an aromatischen Ringen wurde modifiziert. Diese erhalten nun ein „@“ gefolgt von der Ringgröße. Im Folgenden werden aromatische und dreigliedrige Ringe als planare Ringe bezeichnet. Nicht berücksichtigt werden dabei Ringsysteme, die mindestens ein tetraedrisch koordiniertes Atom (z.B. ein $s p^{3}$-hybridisiertes Kohlenstoffatom) enthalten.

Neben Verbesserungen bei der Automatisierung konnte durch den erweiterten Namensraum auch die Genauigkeit der Näherung erhöht werden. Speziell bei planaren Zyklen mit drei bis fünf Ringatomen hat die Erweiterung des Konzepts der Bindungsordnung zu neuen Invariom-Namen geführt. Aus diesen gehen andere Modellverbindungen hervor. Die Modellverbindung eines Invarioms ist das geometrieoptimierte Molekül, aus dem der asphärische Atomformfaktor eines Invarioms abgeleitet wird. Das Vorgehen zum Ableiten der Modellverbindungen wird in Abschnitt 5.3 besprochen. Die Hauptmotivation zur Überarbeitung des Konzepts der Bindungsordnung war die systematische Erweiterung der Invariom-Notation auf organische heteroaromatische Verbindungen und ein erhöhter Grad an Automatisierung bei der Charakterisierung 
atomarer Bindungsumgebungen. Dies verbessert die computergestützte Generierung von Invariom-Namen in den Programmen InvaRIomTool [49] und MoLECOOLQT [72]. Besonders die automatisierte Beschreibung makromolekularer Strukturen, welche DNA- oder RNA-Fragmente enthalten oder heteroaromatische Strukturen als Liganden aufweisen, sind ein zentraler Beweggrund für die bereits umgesetzten aber noch unveröffentlichten Neuerungen. Die konzeptionelle Erweiterung der Bindungsordnung wird im folgenden auf mehrere Beispiele angewendet.

\subsection{Nomenklatur}

Der Invariom-Name setzt sich aus dem Elementsymbol, gefolgt von Bindungsordnung und Elementsymbolen von nächsten Bindungspartnern (in Kleinbuchstaben) zusammen. Die Bindungspartner werden zuerst nach der Bindungsordnung und bei Übereinstimmung nach der Ordnungszahl sortiert. Je höher die Bindungsordnung und je schwerer das Nachbaratom, desto weiter vorne steht es im Invariom-Namen. Bei allen mesomeren Bindungen $(\mathrm{BO}=1.5$ oder \#n; n=3-7) folgen in eckigen Klammern die übernächsten Nachbaratome. Hier wird also die Näherung der übernächsten Nachbaratome (NüN) verwendet [69]. Bei allen Substituenten an planaren Ringsystemen ( $\mathrm{BO}=@ \mathrm{n} ; \mathrm{n}=3-7)$ und bei Einfachbindungen $(\mathrm{BO}=1)$ und Doppelbindungen $(\mathrm{BO}=2)$ werden nur direkt gebundene Nachbaratome aufgeführt. In diesem Fall gilt die Näherung der nächsten Nachbaratome (NnN). Eine Ausnahme der obigen Regeln stellen Einfachbindungen zu den Elementen Schwefel und Phosphor dar, bei denen auch die übernächsten Nachbaratome (NüN) betrachtet werden. Für Wasserstoffatome mit der $\mathrm{BO}=1$ werden ebenfalls übernächste Nachbaratome betrachtet (NüN).

\subsubsection{Präfix}

In manchen Fällen wird als zusätzliche Spezifizierung vor das Elementsymbol ein Präfix geschrieben, welches immer von einem „-“ gefolgt wird. Diese Sonderfälle werden nun anhand von Beispiel erläutert, um die Regeln der Invariom-Nomenklatur zu veranschaulichen. In den Strukturformeln sind die dem Invariom-Namen entsprechenden Atome mit einem roten Kreis markiert und alle explizit im Invariom-Namen enthaltenen Atome blau eingefärbt. $\mathrm{Zu}$ 
jeder Modellverbindung sind der Invariom-Name sowie die linearen Notationen der Moleküle nach SMILES und IUPAC angegeben. Die verwendeten Beispiele sind gleichzeitig die zugehörigen Modellverbindungen des jeweiligen Invarioms. Die Regeln zur Ableitung von Modellverbindungen werden in Abschnitt 5.3 auf Seite 43 erläutert.

\section{Absolute Konfiguration}

Entsprechend der CIP-Nomenklatur wird die absolute Konfiguration bei vier unterschiedlichen Substituenten durch die Präfixe " $R$ “ oder " $S$ “ gekennzeichnet. Die Unterscheidung der absoluten Konfiguration erfolgt aber nur, wenn sich die vier unmittelbar benachbarten Atome unterscheiden. Im Gegensatz zur CIP-Nomenklatur wird nicht das ganze Molekül betrachtet, sondern nur das im Invariom-Namen enthaltene Fragment.

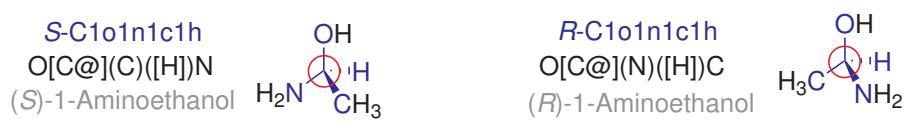

Abbildung 5.1: Die Invariome $S-\mathrm{C} 1 \mathrm{oln} 1 \mathrm{c} 1 \mathrm{~h}$ (links) und $R-\mathrm{C} 1 \mathrm{oln} 1 \mathrm{c} 1 \mathrm{~h}$ (rechts) als Beispiele für absolute Konfiguration in einer Bindungsumgebung.

\section{Ringgröße}

Bei aromatischen und dreigliedrigen Ringen wird die Anzahl der Ringatome als Präfix angegeben.

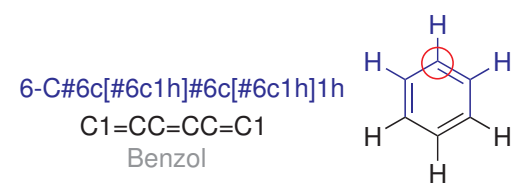

Abbildung 5.2: Das Invariom 6-C\#6c[\#6c1h]\#6c[\#6c1h]1h als Beispiel für eine aromatische Bindungsumgebung.

Im Fall mehrerer zusammenhängender Ringe werden die Ringgrößen aller Zyklen aufgeführt, an denen das Atom beteiligt ist. Die so vorgenommene Unterscheidung ermöglicht die genaue Kategorisierung der Ringgröße, von der die 
Winkel zwischen den Atomen abhängen. Da im Hansen \& Coppens-Multipolmodell starre Pseudoatome verwendet werden, ist der Bindungswinkel für die Übertragbarkeit von Invariomen ebenfalls von Bedeutung.

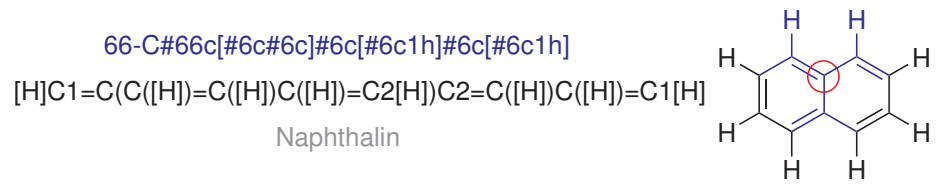

Abbildung 5.3: Das Invariom 66-C\#66c[\#6c\#6c]\#6c[\#6c1h]\#6c[\#6c1h] als Beispiel für eine bicyclische aromatische Bindungsumgebung.

\section{Planarität}

Aufgrund der hohen Flexibilität von Stickstoffatomen erhalten einfach gebundene, jedoch planar koordinierte Stickstoffatome ein „=“ als Präfix.

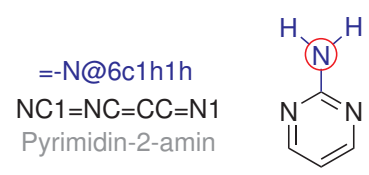

Abbildung 5.4: Das Invariom =-N@6c1h $1 \mathrm{~h}$ als Beispiel für eine planare Bindungsumgebung.

\subsection{Ableiten von Modellverbindungen}

Wie eingangs erwähnt, werden durch die Invariom-Notation keine Moleküle, sondern Bindungsumgebungen von Atomen beschrieben. Deshalb muss die Auswahl der Modellverbindungen zur Erzeugung von Datenbankeinträgen nach bestimmten Kriterien erfolgen. Alle im Invariom-Namen angegebenen Bindungsordnungen und Atome werden zunächst streng erfüllt. Bei aliphatischen Bindungsumgebungen werden alle freien Valenzen der ersten oder zweiten Nachbaratome im Anschluss mit Wasserstoffatomen gesättigt. Die freien Valenzen von aromatischen Ringatomen werden so gesättigt, dass die HückelRegel für delokalisierte $\pi$-Elektronen erfüllt ist (siehe Gleichung 5.2).

$$
\pi e^{-}=4 n+2(n=0,1,2, \ldots)
$$


Als zusätzliches Kriterium werden so wenig Atome wie möglich ergänzt, da aus Gründen der Rechenzeit immer die kleinst mögliche Modellverbindung erstellt werden soll. Anschließend werden alle freien Valenzen unter Beibehaltung der $\pi$-Elektronen mit Wasserstoffatomen gesättigt. In manchen Fällen sind trotz der oben beschriebenen Regeln mehrere Stellungsisomere möglich. Wenn der Invariom-Name das Regioisomer ${ }^{1}$ nicht eindeutig spezifiziert, werden alle in Frage kommenden Regioisomere berechnet. Als Modellverbindung des betreffenden Invarioms wird anschließend das Isomer mit dem kleinsten Dipolmoment oder der geringsten Energie ausgewählt. Details zur Auswahl der Modellverbindung werden im Abschnitt 5.5 auf Seite 46 erläutert. Beispiele für Invariome und ihre zugehörigen Modellverbindungen wurden bereits in Abschnitt 5.2.1 vorgestellt. In Kapitel 6 werden die Modellverbindungen für DNA-Nukleoside vorgestellt.

\subsection{Erstellen von Invariomen}

\subsubsection{Geometrieoptimierung}

Für die aus dem Invariom-Namen abgeleitete molekulare Strukturformel wird eine Eingabedatei für das Programm GaUssian03 [73] im Z-Matrix-Format erstellt. Dafür hat sich das Programm Avogadro [74] als besonders geeignet herausgestellt. Hier kann eine Struktur in Form des SMILES-Codes [75] eingegeben und mit dem Kraftfeld UFF (Universal Force Field) [76] eine Startgeometrie erzeugt werden. Es ist wichtig die Geometrieoptimierung mit einer guten Startgeometrie zu beginnen, da es der rechenintensivste und zeitaufwendigste Teil der Prozedur ist. Die Geometrieoptimierung wird mit dem Funktional B3LYP der Dichtefunktionaltheorie durchgeführt [77, 78]. Bei Molekülen, die kein schwereres Element als Argon enthalten, wird mit dem Basissatz 6-31g* voroptimiert. Mit der Geometrie aus der Voroptimierung wird anschließend mit dem Basissatz D95++(3df,3pd) nach Dunning [79] eine weitere Geometrieoptimierung vorgenommen, bei der als Konvergenzkriterium $10^{-9}$ au (very tight) verwendet wird. Die Elektronendichte des Moleküls wird in Form einer FChkDatei ausgegeben. Zur Verifizierung des Energieminimums wird zusätzlich eine Frequenzberechnung durchgeführt.

\footnotetext{
${ }^{1}$ Regioisomerie ist eine Untergruppe der Konstitutionsisomerie. Die Bezeichnungen Stellungsisomerie und Ortsisomerie werden gleichbedeutend mit Regioisomerie verwendet.
} 


\subsubsection{Theoretische Strukturfaktoren}

Mit dem Programm TONTO können Beugungsdaten theoretisch simuliert werden. Dazu wird eine Elementarzelle der Raumgruppe P $\overline{1}$ und den Gitterkonstanten von $a, b, c=30 \AA$ und $\alpha, \beta, \gamma=90^{\circ}$ definiert. Das Molekül wird mit dem Ursprung der theoretischen Rechnung auf den fraktionellen Koordinaten $\frac{1}{4}, \frac{1}{4}, \frac{1}{4}$ platziert. Durch Fouriertransformation der (quantenmechanischen) molekularen Elektronendichte werden nun Intensitäten für Miller-Indices (h,k,l) von 0 bis 40 berechnet. $^{2}$

\subsubsection{Multipolprojektion}

Anschließend wird ein Multipolmodell aufgesetzt. Das Modell ist bis auf einige technische Details analog zur Modellierung experimenteller Daten im Multipolformalismus. Dabei sollen nur so viele Parameter verfeinert werden, wie notwendig sind, um die theoretischen Daten im Rahmen der Fehler reproduzieren zu können. Anders als bei experimentellen Daten wird hier auch für Wasserstoffatome die Multipol-Expansion bis zum Hexadecapol-Level $(l=4)$ durchgeführt. Für die manuelle Anpassung lokaler Koordinatensysteme und atomarer Punktgruppensymmetrie wird das Programm MolecoolQT [72] verwendet. Bei gleichen Bindungsumgebungen bzw. Invariom-Namen wird als zusätzliche Bedingung chemische Gleichheit in Form sog. „chemical constraints“ definiert. Zusätzlich wird für alle Atome mit dem selben Invariom-Namen nur ein $\kappa$-Parameter eingeführt. Die theoretischen Beugungsdaten werden mit dem Programmteil XDLSM des XD-Programmpakets verfeinert. Probleme bei der Phasenbestimmung werden an dieser Stelle durch Verwendung der Raumgruppe $\mathrm{P} \overline{1}$ vermieden, da die Phasenwinkel $\phi$ der Reflexe zentrosymmetrischer Raumgruppen nur Werte von $0^{\circ}$ oder $180^{\circ}$ annehmen können.

Nach erfolgreicher Verfeinerung ist die Multipolprojektion [80] der molekularen Elektronendichte aus der Geometrieoptimierung abgeschlossen. Die resultierenden atomzentrierten Elektronendichtefragmente können nun als Streufaktoren für den Aufbau der Invariom-Datenbank verwendet werden.

\footnotetext{
${ }^{2}$ Prinzipiell ist die Berechnung beliebig hoher Miller-Indices möglich.
} 


\subsection{Invariom-Datenbank}

Der Aufbau der Datenbank aus den fertigen Modellverbindungen erfolgt vollautomatisch mit Hilfe eines Skripts. Im Gegensatz zu anderen Datenbanken wird für die Einträge der Invariom-Datenbank prinzipiell nicht über verschiedene Modellverbindungen gemittelt. Durch das Hinzufügen neuer Modellverbindungen werden die bestehende Datenbankeinträge also nicht verändert. Statt dessen wird die Datenbank modular um zusätzliche Bindungsumgebungen ergänzt.

\subsubsection{Inhalt der Einträge}

In der Invariom-Datenbank werden alle atomaren Elektronendichtefragmente gespeichert. Jeder Eintrag besteht aus Invariom-Name, Multipol- und $\kappa^{-}$ Parametern, der relativen Zuordnung des lokalen Koordinatensystems sowie der lokalen, atomaren Punktgruppensymmetrie nach Hermann-Maugin. Um die Reproduzierbarkeit der Einträge zu gewährleisten, wird auch der IUPACoder Trivialname der zugehörigen Modellverbindung gespeichert.

\subsubsection{Minimal-Kriterien}

Gibt es zu einem Invariom-Namen mehrere Modellverbindungen, wird nach den Minimal-Kriterien eine der Modellverbindungen für den Datenbankeintrag ausgewählt. In folgender Reihenfolge werden die Minimal-Kriterien auf alle in Frage kommenden Modellverbindungen angewendet, bis nur noch eine Modellverbindung übrig bleibt:

1. Die Modellverbindung mit der geringsten Ladung wird gegenüber einer höher geladenen bevorzugt.

2. Die Modellverbindung mit dem geringsten Penalty ${ }^{3}$ wird bevorzugt.

3. Die Modellverbindung mit der geringsten Anzahl an Elektronen wird bevorzugt.

\footnotetext{
${ }^{3}$ Der Penalty ist ähnlich der Summe der Ordnungszahlen aller Atome, wobei für Wasserstoffatome der Wert '0', für Kohlenstoffe der Wert '1', für Stickstoffe der Wert '3' und für Sauerstoffatome der Wert '2' verwendet wird. Mit dem PENALTY werden kohlenstoffbasierte Strukturen den entsprechenden Bor-Analoga vorgezogen. Zusätzlich werden Sauerstoffatome gegenüber Stickstoffatomen bevorzugt.
} 
4. Die Modellverbindung mit der geringsten Atomanzahl wird bevorzugt.

5. Die Modellverbindung mit dem geringsten Dipolmoment wird bevorzugt.

6. Die Modellverbindung mit der geringsten absoluten Energie nach HF wird bevorzugt.

In den Kapiteln 6 und 8 werden die Modellverbindungen von DNA-Nukleosiden und Fluorochinolonen vorgestellt, die aus den Minimal-Kriterien resultieren.

\subsection{Invariom-Transfer}

Nach dem Invariom-Ansatz handelt es sich bei jedem Invariom um ein „transferierbares atomares Elektronendichtefragment“ (TAD). Für jedes Atom eines Zielmoleküls muss ein entsprechender Datenbankeintrag vorhanden sein. Der Transfer von Datenbankeinträgen auf ein Zielmolekül kann mit dem Programm INVARIOMTOOL [49] und seit kurzem auch mit dem Programm MolEcoolQT [72] erfolgen.

\subsubsection{InvariomTool}

INVARIOMTOOL [49] ist bislang speziell auf das XD-Programmpaket [46] zugeschnitten und dient als Präprozessor für selbiges Paket. Als Eingabe werden die XD-Dateien $x$ d.mas und $x$ d.res gelesen. Nach dem dem Aufruf von INVARIOMTOOL und dem Invariom-Transfer enthält die Datei $x d$.res.invariomtool die transferierten Multipol-und $\kappa$-Parameter aus der Invariom-Datenbank. Die Datei $x d$.mas.invariomtool enthält außerdem die Zuordnungen der lokalen Koordinatensysteme. Direkt nach dem Transfer kann eine Invariom-Verfeinerung mit XDLSM durchgeführt werden. Dabei werden, analog zu einer Verfeinerung mit sphärischen Streufaktoren in SHELXL, in der Regel nur Atompositionen und Auslenkungsparameter verfeinert. Mit der vom InVARIOMTOOL ausgeschriebenen Datei $x d$.mas.HasSameSymm kann zusätzlich eine automatisierte MultipolVerfeinerung durchgeführt werden, wenn die strengen Kriterien an die Auflösung und die Datenqualität erfüllt werden. Für Multipol- und $\kappa$-Parameter aller Atome mit dem selben Invariom-Namen werden dabei zur Reduzierung der Anzahl an Parametern bereits „chemical constraints“ eingeführt, und damit chemische Gleichheit definiert. Es werden für jedes Atom auch nur die Multipol-Parameter verfeinert, welche zur Erzeugung des jeweiligen Invarioms 
entsprechend der lokalen atomaren Punktgruppensymmetrie verwendet wurden (siehe Abschnitt 5.4.3).

\subsubsection{MolecoolQt}

MolecoolQT [72] hat einen speziellen Invariom-Modus, welcher zahlreiche Vorteile bietet. Der Invariom-Transfer kann hier ausgehend von einer SHELXLVerfeinerung [43] erfolgen und Invariom-Transfer-Dateien für asphärische Verfeinerungen in den Programmen XD [46] und MOPRO [44, 45] ausschreiben. Durch Einlesen der aktuellen Invariom-Datenbank kann auf einfache Weise überprüft werden, ob alle für das jeweilige Zielmolekül benötigten Invariome in dieser vorhanden sind, bevor der Invariom-Transfer durchgeführt wird.

\section{Semi-automatischer Transfer}

Bei Atomen auf speziellen kristallographischen Lagen oder bei Fehlordnungen erfolgt der Invariom-Transfer mit InVARIOMTOOL nicht vollautomatisch. Hier ist MolecoolQt besonders hilfreich, da es die kristallographische Symmetrie erkennt und Moleküle vor Generierung der Invariom-Namen entsprechend vervollständigt. Wenn Fehlordnung in einer SHELXL-Verfeinerung mit dem PARTBefehl behandelt wurde, kann MolecoolQT [72] die PART-Information auswerten und beim anschließenden Transfer beachten. Auch beim Einlesen einer XD-Datei wird die PART-Information einer im selben Verzeichnis liegenden SHELXL-Datei ausgewertet und in der Datei $x d \_p a r t . a u x$ gespeichert. Die Invariom-Namen können zusätzlich im Invariom-Modus kontrolliert und durch Modifizierung der Bindungsordnungen bearbeitet werden. Für einen Transfer mit INVARIOMTOOL kann die Datei „Invariome.in“ ausgeschrieben werden. Dadurch werden beim erneuten Aufruf von InVARIOMTOol die in der Datei angegebenen Invariome transferiert. Da sich das Programm MolecoolQT [72] zum Zeitpunkt dieser Arbeit noch in der Testphase befindet, wird der InvariomTransfer momentan noch mit InVARIOMTOOL durchgeführt. Nach dem Transfer können schließlich die lokalen Koordinatensysteme in MolecoolQT [72] überprüft und bei Bedarf im XD-Modus angepasst werden. 


\subsection{Vergleichsprüfung zur Transferierbarkeit}

Die im Invariom-Ansatz verwendetet Näherungen wurden bereits in Abschnitt 5.3 auf Seite 43 vorgestellt. Im Hinblick auf die Berechnung und Interpretation molekularer Eigenschaften (siehe Kapitel 3) ist eine Verifizierung der Methode besonders wichtig.

Die Transferierbarkeit und Reproduzierbarkeit der asphärischen Elektronendichte wurde bereits mit Hilfe der bindungstopologischen Parameter und der atomaren Eigenschaften nach Bader [54] für Tripeptide untersucht [81]. Die Genauigkeit des Transfers komplexer chemische Bindungsumgebungen, wie die erst seit kurzem verfügbaren heteroaromatischen Invariome, wurde jedoch noch nicht untersucht. Zur Bestimmung der Güte des Invariom-Models wurde für die in Kapitel 8 diskutierten Fluorochinolon-Antibiotika ein neuartiges Verfahren entwickelt und angewendet. Es basiert darauf, Modellunterschiede in Form einer Differenzelektronendichte zu untersuchen. Es wurde bereits für die Kreuzvalidierung der Invariom-Datenbank mit experimentellen Daten der nichtproteinogenen Aminosäuren L-Ornithin [82] und Aminoisobuttersäure [83] sowie beim Makrolid-Antibiotikum Roxithromycin [66] eingesetzt.

Neben der experimentellen Validierung wird in dem Kapitel 8 noch ein theoriebasiertes Verfahren zur Anwendung kommen. Dafür wird eine sehr rechenintensive Geometrieoptimierung eines Fluorochinolon-Antibiotikums durchgeführt, wobei dieselben Basis [B3LYP/D95++(3df,3pd)] wie zur Erstellung von Invariomen verwendet wird. Es werden theoretische Strukturfaktoren der berechneten Elektronendichte simuliert. Gegen die theoretischen Daten des gesamten Moleküls werden nun eine Invariom-Verfeinerung und eine MultipolVerfeinerung durchgeführt. Bei der Invariom-Verfeinerung wird nur der Skalenfaktor verfeinert. Bei der Multipol-Verfeinerung werden die Multipol- und $\kappa$ Parameter entsprechend der $x$ d.mas.HasSameSymm Datei automatisiert verfeinert. Aus beiden Modellen wird anschließend eine Differenzelektronendichte berechnet, welche die Unterschiede der Modelle abbildet. 


\section{Teil III}

\section{Ergebnisse}



Kapitel 6

Erweiterung der Invariom-Datenbank 


\subsection{Erkennung der Bindungsumgebung}

Der zentrale Beweggrund für die Überarbeitung der Invariom-Notation ist die Verbesserung des Automatisierungsgrades bei der Charakterisierung atomarer Bindungsumgebungen. Dadurch können heute makromolekulare Strukturen, welche DNA- oder RNA-Fragmente enthalten oder heteroaromatische Liganden aufweisen ebenfalls zuverlässig mit asphärischen Streufaktoren verfeinert und chemische Eigenschaften dieser Strukturen berechnet werden. Die dafür notwendige Erweiterung des Konzepts der Bindungsordnung wurde gemeinsam mit Dr. Christian B. Hübschle entwickelt und von ihm in die Programme InvariomTool [49] und MolecoolQT [72] implementiert. In diesem Kapitel wird erläutert, wie die chemische Bindungsumgebung im Fall von aromatischen und dreigliedrigen Ringen durch geometrische Kriterien bestimmt werden kann. Der Invariom-Formalismus wurde bereits in Kapitel 5 eingeführt, wo auch die Invariom-Nomenklatur vorgestellt wurde.

\subsubsection{Planarität}

Aus den Differenzen des Ortsvektors eines Atoms $\vec{o}_{A}$ mit denen seiner Nachbaratome $\vec{o}_{i}$ werden nach Gleichung 6.1 die Bindungsvektoren $\vec{b}_{i}$ berechnet. Dabei steht $l$ für die Anzahl an Substituenten.

$$
\left[\vec{b}_{i}\right]_{1}^{l}=\vec{o}_{A}-\vec{o}_{i}
$$

Aus je zwei Bindungsvektoren $\vec{b}_{i}$ und $\vec{b}_{i+1}$ wird das Kreuzprodukt berechnet und der resultierende Vektor normiert. Man erhält nach Gleichung 6.2 genau $l$ Vektoren $\vec{n}_{i}$, die auf der von den Bindungsvektoren aufgespannten Ebene senkrecht stehen. Der Vektor $\vec{n}_{l}$ wird dabei aus den Bindungsvektoren $\vec{b}_{l}$ und $\vec{b}_{1}$ berechnet.

$$
\left[\vec{n}_{i}\right]_{1}^{l-1}=\frac{\vec{b}_{i} \times \vec{b}_{i+1}}{\left|\vec{b}_{i} \times \vec{b}_{i+1}\right|} \quad \vec{n}_{l}=\frac{\vec{b}_{l} \times \vec{b}_{1}}{\left|\vec{b}_{l} \times \vec{b}_{1}\right|}
$$

Aus allen $l$ Kombinationen der Bindungsnormalen eines Atoms werden anschliessend die Skalarprodukte berechnet und miteinander multipliziert. Da- 
durch ergibt sich der Planaritätswert $(P W)$ eines Atoms.

$$
P W=1 \cdot \prod_{i=1}^{l-1}\left(\prod_{j=i+1}^{l}\left|\vec{n}_{i} \cdot \vec{n}_{j}\right|\right)
$$

Im Falle von drei kovalenten Bindungen ergibt sich der Planaritätswert nach Gleichung 6.3 wie folgt:

$$
P W_{l=3}=1 \cdot\left|\vec{n}_{1} \cdot \vec{n}_{2}\right| \cdot\left|\vec{n}_{1} \cdot \vec{n}_{3}\right| \cdot\left|\vec{n}_{2} \cdot \vec{n}_{3}\right|
$$

Für exakt planar koordinierte Atome und linear koordinierte Atome ist der $P W$ „1“ und bei nicht planar sondern beispielsweise tetraedrisch oder oktaedrisch koordinierten Atomen „O“.

\subsubsection{Aromatische Ringe}

Für die Erkennung größerer, aromatischer Ringsysteme werden alle Atome, die mindestens zwei Nachbaratome haben und deren Planaritätswert oberhalb des Schwellenwerts $(P W>0,80)$ liegt, in eine Liste geschrieben.

Anschließend wird nach Gleichung 6.4 aus jedem Tripel von Atomen der Bindungswinkel $\varphi$ berechnet.

$$
\varphi=\arccos \left(\frac{\vec{b}_{i}}{\left|\vec{b}_{i}\right|} \cdot \frac{\vec{b}_{i+1}}{\left|\vec{b}_{i+1}\right|}\right)
$$

Wie in Abbildung 6.1 dargestellt wird durch Linerakombination der Bindungsvektoren $\vec{b}_{i}$ und $\vec{b}_{i+1}$ nach Gleichung 6.5 der winkelhalbierende Vektor $\vec{d}_{i}$ erzeugt.

$$
\vec{d}_{i}=\vec{b}_{i}+\vec{b}_{i+1}
$$

Wenn es sich bei dem Atomtripel um Mitglieder eines aromatischen Ringsystems handelt, erhält man durch Multiplikation von $\overrightarrow{d_{i}}$ mit einem Skalierungsparameter $s$ nach Gleichung 6.6 einen Vektor, der vom betrachteten Atom zum Ringmittelpunkt $\vec{m}$ zeigt,

$$
\vec{m}=\vec{d}_{i} \cdot s
$$




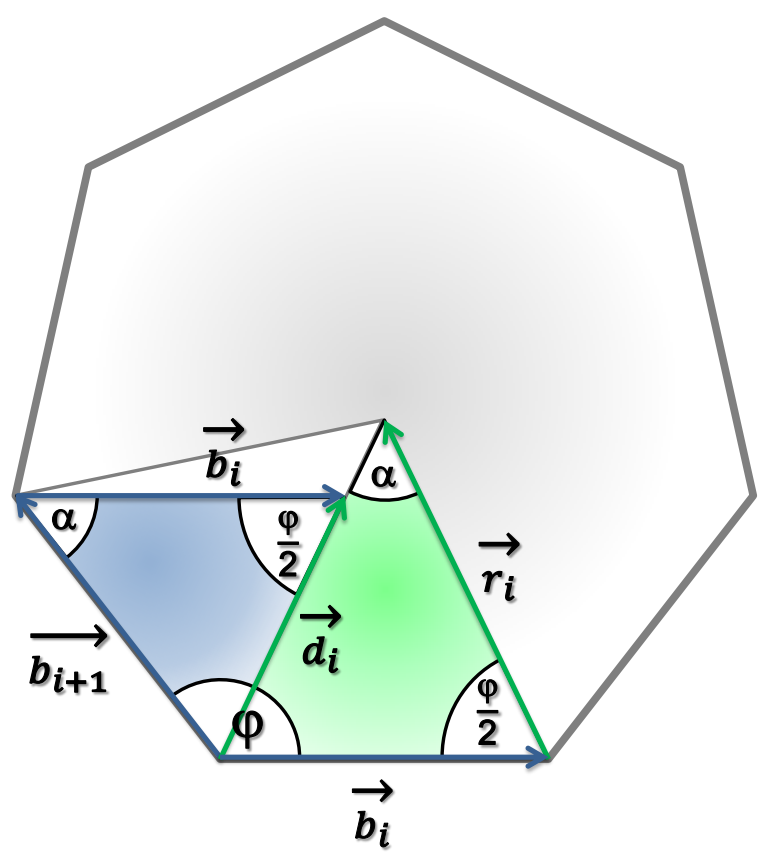

Abbildung 6.1: Schema zur Erkennung aromatischer Ringe am Beispiel eines siebengliedrigen Rings. Das Verfahren ist für planare Ringe beliebiger Größe anwendbar.

Um den Skalierungsparameter $s$ allgemeingültig berechnen zu können wird näherungsweise angenommen, dass es sich bei den aromatischen Ringen um regelmäßige Vielecke handelt. Dadurch ergibt sich ein gleichseitiges Dreieck mit dem Winkel $\alpha$ und zweimal dem Winkel $\frac{\varphi}{2}$ (siehe Abbildung 6.1). Da die Winkelsumme des Dreiecks $180^{\circ}$ beträgt, kann nach Gleichung $6.7 \alpha$ durch $\varphi$ ausgedrückt werden.

$$
\begin{aligned}
& \alpha=180^{\circ}-2 \cdot \frac{\varphi}{2} \\
& \alpha=180^{\circ}-\varphi
\end{aligned}
$$

Der Sinus von $\alpha$ kann nun durch Verwendung der Symmetrie der Sinusfunktion in den Sinus von $\varphi$ überführt werden.

$$
\begin{aligned}
& \sin (\alpha)=\sin (180-\varphi) \\
& \sin (\alpha)=-\sin (\varphi-180) \\
& \sin (\alpha)=\sin (\phi)
\end{aligned}
$$


In den Gleichungen 6.9 und 6.10 werden $\vec{r}_{i}$ und $\vec{d}_{i}$ durch Anwendung des Sinussatzes durch den Bindungswinkel $\varphi$ und die Bindungslänge $\left|\vec{b}_{i}\right|$ ausgedrückt. Dabei wurde statt $\sin \alpha$ nach Gleichung 6.8 durch $\sin \varphi$ eingesetzt.

$$
\begin{gathered}
\frac{\left|\vec{r}_{i}\right|}{\sin \left(\frac{\varphi}{2}\right)}=\frac{\left|\vec{b}_{i}\right|}{\sin \varphi} \\
\frac{\left|\vec{d}_{i}\right|}{\sin \varphi}=\frac{\left|\vec{b}_{i}\right|}{\sin \left(\frac{\varphi}{2}\right)}
\end{gathered}
$$

Der Skalierungsparamter $s$ wird nach Gleichung 6.11 aus dem Verhältnis von $\left|\vec{r}_{i}\right|$ und $\left|\vec{d}_{i}\right|$ berechnet. Durch Einsetzen der Gleichungen 6.9 und 6.10 wird $s$ nur in Abhängigkeit des Bindungswinkels $\varphi$ erhalten.

$$
\begin{aligned}
& s=\frac{\left|\vec{r}_{i}\right|}{\left|\vec{d}_{i}\right|} \\
& s=\frac{\left|\vec{b}_{i}\right| \cdot \sin \left(\frac{\varphi}{2}\right)}{\sin \varphi} \cdot \frac{\sin \left(\frac{\varphi}{2}\right)}{\left|\vec{b}_{i}\right| \cdot \sin \varphi} \\
& s=\frac{\sin ^{2}\left(\frac{\varphi}{2}\right)}{\sin ^{2} \varphi}
\end{aligned}
$$

Nun kann der Abstand anderer Atome zum möglichen Ringelmittelpunkt $\vec{m}$ berechnet werden. Atome, deren Abstand zum Ringmittelpunkt kleiner als $1,4 \cdot\left|\vec{r}_{i}\right|$ ist, sind Teil des selben Rings. Als zusätzliches Kriterium kann überprüft werden, ob die Anzahl an Ringatomen dem berechneten Erwartungswert entspricht. Mit der Näherung eines gleichseitigen Vielecks kann der Erwartungswert der Ringgröße $n$ aus dem Bindungswinkel $\varphi$ berechnet werden.

$$
n=\frac{360^{\circ}}{\left(180^{\circ}-\varphi\right)}
$$

\subsubsection{Dreigliedrige Ringe}

Ein Atom ist Teil eines dreigliedrigen Rings, wenn es der nächste Nachbar eines seiner übernächsten Nachbaratome ist. Zur Erkennung wird für jedes Atom eine Liste mit den Namen aller nächsten Nachbaratome erstellt und überprüft, 
ob auf der Liste eines übernächsten Nachbars der eigene Name steht. Ist dies der Fall, so wurde ein dreigliedriger Ring entdeckt.

\subsection{Automatisierte Erzeugung von Invariomen}

Zur Beschleunigung des Ablaufs wurden die zentralen Schritte zur Erzeugung der Invariome im Rahmen dieser Arbeit automatisiert. Dazu wurde das ShellSkript „setup_both“ geschrieben. Der hohe Grad an Automatisierung dient der Vermeidung von Fehlern beim Aufsetzten des Modells und reduziert den Aufwand bei den Arbeitsabläufen erheblich. Dadurch kann die Invariom-Datenbank heute mit geringem Einsatz von „Humankapital“ bei vorhandener Rechenkapazität innerhalb kürzester Zeit um zahlreiche Bindungsumgebungen erweitert werden. Zur Erzeugung eines Invarioms muss nur noch die Modellverbindung erstellt und das Multipolmodell ausschließlich für die neuen Invariome in dieser Modellverbindung von Hand vorgenommen werden.

Die Invariom-Datenbank befindet sich in einem beschleunigten Aufbauprozess und wird fortlaufend erweitert und optimiert. Aktuell wird untersucht, inwiefern Bindungsumgebungen metallorganischer Komplexe mit dem InvariomFormalismus beschrieben und in die Datenbank eingebracht werden können.

\subsubsection{Das Skript „setup_both“}

Ausgehend von der Eingabedatei für GAUSSIAN [73] werden alle Schritte zur Erzeugung der Invariome chronologisch und ohne Zeitverlust durchgeführt. Die benötigte Ordnerstruktur wird angelegt und eine Voroptimierung mit kleiner Basis [B3LYP/6-31g*] in GAUSSIAN [73] durchführt. Die verbesserte Geometrie der Voroptimierung wird anschließend mit Hilfe des Programms OpEN BABEL $[84,85]$ als Startpunkt für die folgende Optimierung verwendet. Dadurch verkürzt sich die Rechenzeit erheblich. Mit dem bereits zu Beginn der Doktorarbeit vorliegenden Programm Fchk2tonto wird eine Eingabedatei (stdin) für TONTO [52] geschrieben, um theoretische Strukturfaktoren zu berechnen. Ebenso werden die Atompositionen aus der Geometrieoptimierung als Eingabedatei für XD ( $x$ d_ini.inp) ausgeschrieben. Auch das bereits vorliegende Programm tontostdout2xdhkl zum Konvertieren der von TONTO [52] berechneten theoretischen Strukturfaktoren in die xd.hkl Datei wird in „setup_both“ aufgerufen.

Ein Startmodell für die Multipolprojektion wird mit INVARIOMTOOL generiert. 
Die Datei $x$ d.mas.HasSameSymm enthält lokale Koordinatensysteme, atomare Punktgruppensymmetrie und Zuordnungen der $\kappa$-Parameter für alle bereits in der Datenbank vorhandenen Invariome. Der Invariom-Transfer ist in Abschnitt 5.6 auf Seite 47 beschrieben. Besondere Spezifikationen für die Verfeinerung gegen theoretischen Daten werden mit SED, dem „UNIX Stream Editor" in die Kontrolldatei $x$ d.mas eingetragen. Der Korrekturterm für anormale Dispersion wird ausgeschaltet und das Kriterium für beobachtete Reflexe von $I>3 \cdot \sigma$ auf $I>0 \cdot \sigma$ modifiziert, damit alle theoretischen Strukturfaktoren in der Verfeinerung verwendet werden. Die Verfeinerung von Atompositionen und Auslenkungsparametern wird deaktiviert, da die Atompositionen aus der Theorie verwendet werden und in den theoretischen Strukturfaktoren keine thermische Bewegung enthalten ist. Für die Multipolprojektion müssen nur Koordinatensysteme, lokale Symmetrie und $\kappa$-Parameter der neuen Invariome manuell angepasst werden.

Der routinemäßige Transfer aller vorhandenen Datenbankeinträge auf theoretische Daten hat sich als ideales Testfeld für die Datenbank und das Programm INVARIOMTOOL herausgestellt, wodurch bereits Fehler im Multipolmodell von Modellverbindungen entdeckt und korrigiert werden konnten.

\subsection{Anwendung auf Heteroaromaten}

Für die fünf Nukleoside von RNA und DNA sowie neun verschieden substituierte Fluorochinolon-Antibiotika wurden nach dem in Kapitel 5 auf Seite 44 beschriebenen Verfahren Datenbankeinträge erzeugt. Die für die Ringatome verwendeten Modellverbindungen sollen hier vorgestellt werden. Für jedes Zielmolekül wird der Zusammenhang zwischen dem Invariom-Namen und der zugehörigen Modellverbindung in einem Transfer-Diagramm gezeigt. In den Abbildungen 6.3 bis 6.7 sind die Modellverbindungen kreisförmig um das jeweils zentral positionierte Zielmolekül verteilt. Die aus den Modellverbindungen transferierten Invariome sind mit einem roten Kreis markiert und alle explizit im Invariom-Namen enthaltenen Atome blau eingefärbt. Der InvariomName und die linearen Notationen der Moleküle nach SMILEs [75] sowie IUPAC ${ }^{1}$ sind zu jeder Modellverbindung angegeben.

\footnotetext{
${ }^{1}$ Es wird statt der deutschen die englische IUPAC-Nomenklatur verwendet, da die Modellverbindungen auch in der Datenbank nach englischer IUPAC-Nomenklatur benannt sind.
} 


\subsubsection{Nukleoside}

Die DNA-Nukleoside Adenosin (A), Guanosin (G), Cytidin (C) und Thymidin (T) sowie das nur in der RNA vorkommende Uridin (U) sind aufgrund ihrer biochemischen Funktion als Speicher der Erbinformation medizinisch besonders interessant. Sie sind neben den Peptiden ein häufiger Bestandteil makromolekularer Strukturen. Adenosin (A) und Guanosin (G) basieren auf der Purin-Struktur; Cytidin (C), Thymidin (T) und Uridin (U) auf dem PyrimidinGrundgerüst. Die $\beta$-D-Ribofuranose (D-Ribose) verbindet die Nukleinbasen mit den

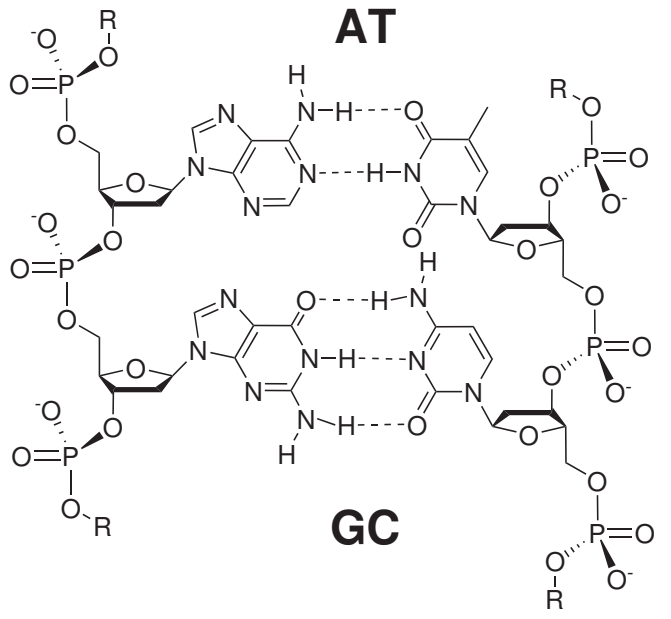

Abbildung 6.2: Ausschnitt aus der DNAStruktur. Die Nukleinbasen Adenin, Thymin, Guanin und Cytosin sind über DRibose mit der Phosphatkette verknüpft. Es sind exemplarisch die Basenpaare AT (oben) und GC (unten) abgebildet.
Phosphatketten von RNA und DNA. Sie wird bei der Invariombetrachtung mit $R$ abgekürzt. Die Atome des Ringsystems der mit $\beta$ D-Ribofuranose substituierten $\mathrm{Nu}-$ kleinbasen stimmen in der InvariomNäherung mit denen der Methylsubstituierten Basen überein. Die Stickstofftome der Amingruppen sind in den Basenpaaren AT (Adenosin, Thymidin) und GC (Guanosin und Cytidin) nicht tetraedrisch sondern planar koordiniert. Die planar koordinierten Stickstoffatome sind $s p^{2}$ statt $s p^{3}$-hybridisiert. Um diesem in Bezug auf die atomare Elektronendichteverteilung gravierenden Unterschied gebührend Rechnung zu tragen, werden die Invariom-Namen von planaren Stickstoffatomen mit dem Präfix „=-“ versehen (siehe Abschnitt 5.2.1 auf Seite 41). Im Invariom-Ansatz werden zur Beschreibung aller Ringatome der fünf Nukleoside insgesamt 21 verschiedene Modellverbindungen verwendet. Es wäre zwar möglich alle vier Basen zu berechnen und die asphärischen Atomformfaktoren von dort zu transferieren; das hier gewählte Verfahren hat aber den Vorteil, dass es konsistent auf beliebige heteroaromatische Verbindungen angewendet werden kann. Durch die 21 Modellverbindungen werden darüber hinaus eine Reihe von Bindungsumgebungen anderer Moleküle mit abgedeckt. 


\section{Adenosin (A)}

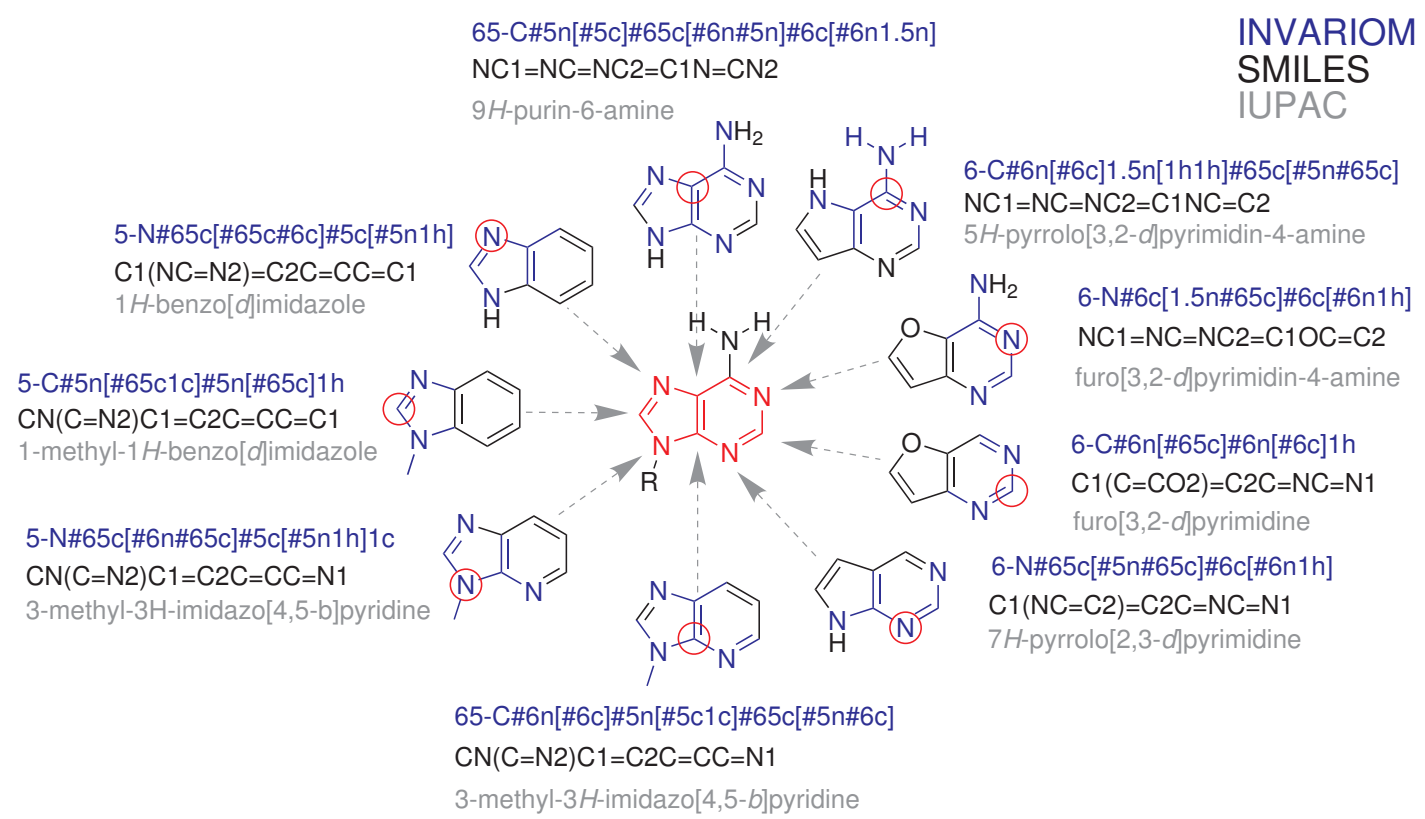

Abbildung 6.3: Modellverbindungen für Adenosin, die im InvariomAnsatz zur Rekonstruktion der molekularen Elektronendichte aus asphärischen Atomformfaktoren verwendet werden.

\section{Thymidin (T)}

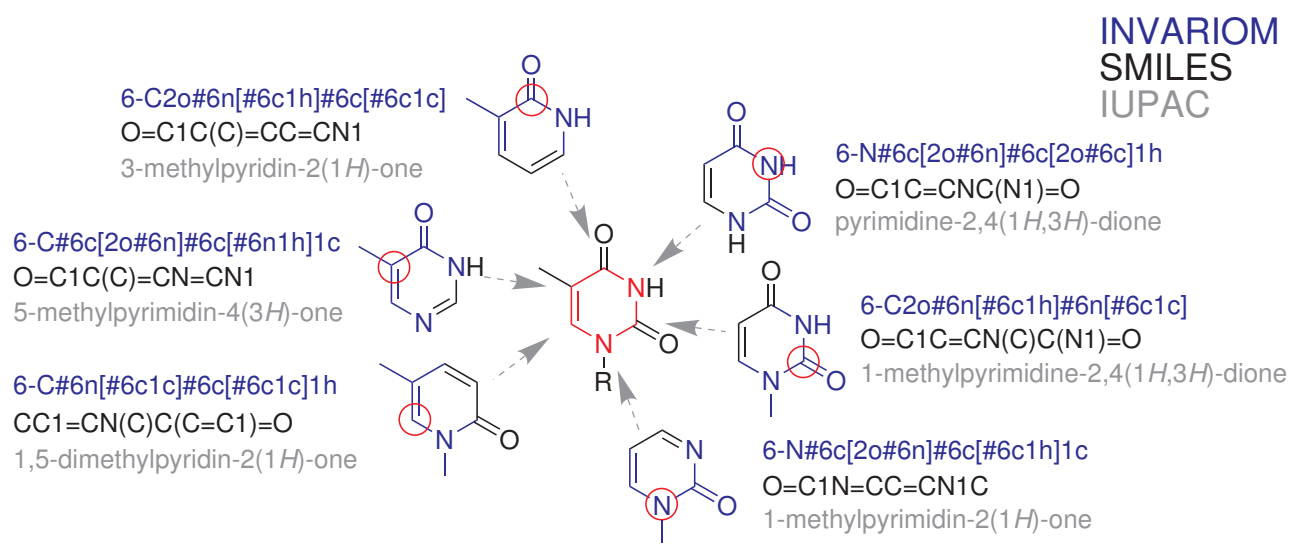

Abbildung 6.4: Modellverbindungen für Thymidin, die im InvariomAnsatz zur Rekonstruktion der molekularen Elektronendichte aus asphärischen Atomformfaktoren verwendet werden. 


\section{Guanosin (G)}

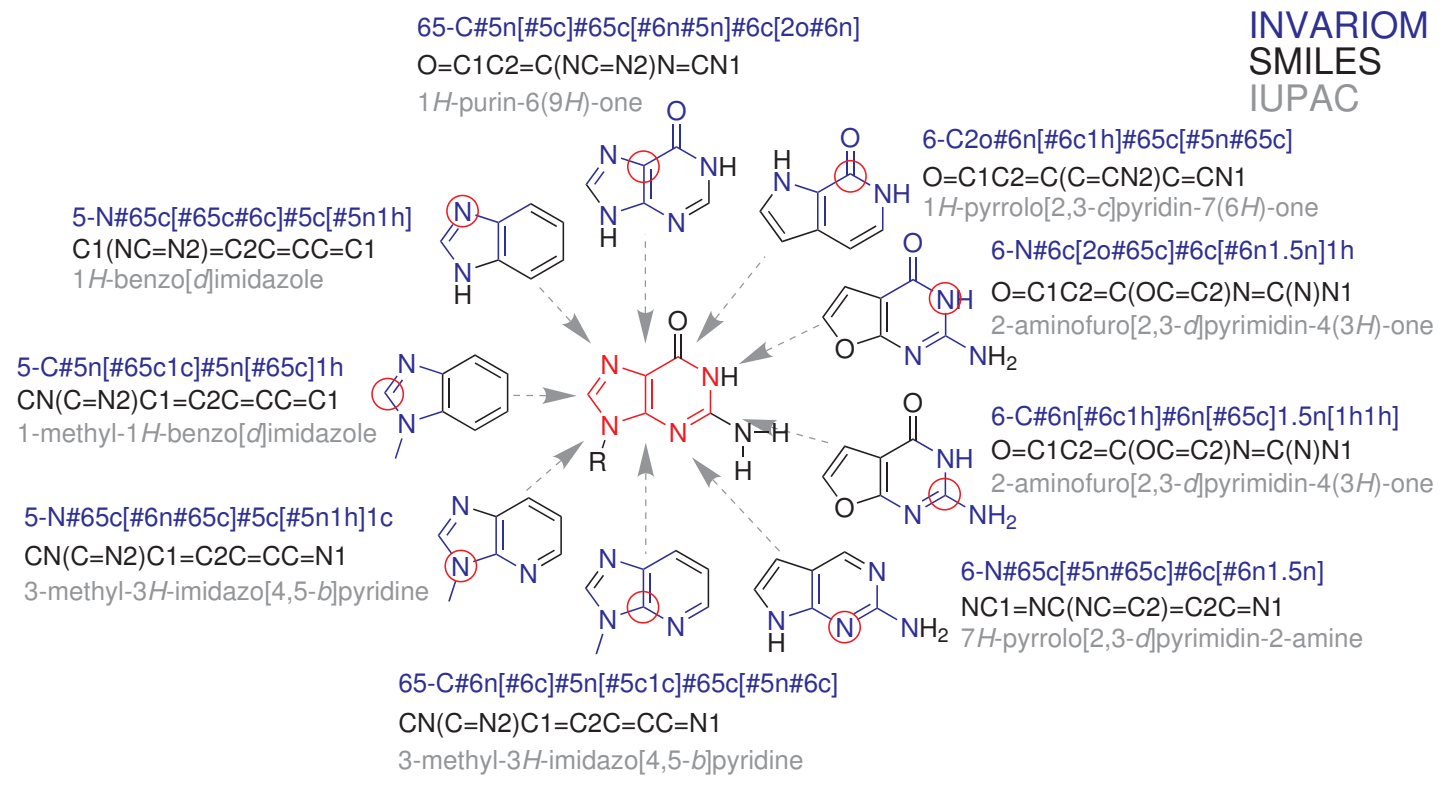

Abbildung 6.5: Modellverbindungen für Guanosin, die im InvariomAnsatz zur Rekonstruktion der molekularen Elektronendichte aus asphärischen Atomformfaktoren verwendet werden.

\section{Cytidin (C)}

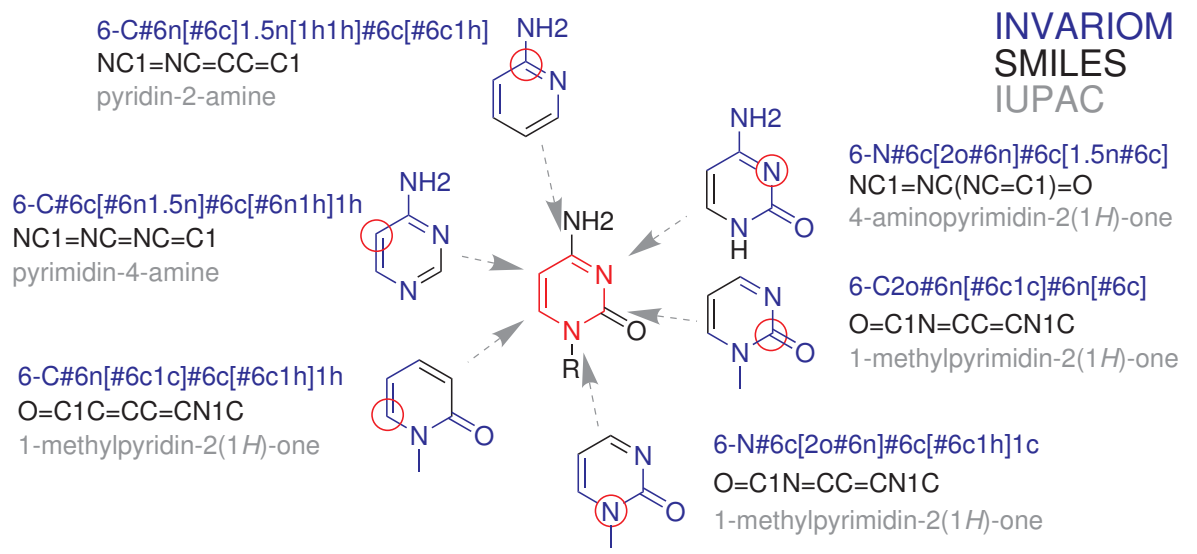

Abbildung 6.6: Modellverbindungen für Cytidin, die im Invariom-Ansatz zur Rekonstruktion der molekularen Elektronendichte aus asphärischen Atomformfaktoren verwendet werden. 


\section{Uridin (U)}

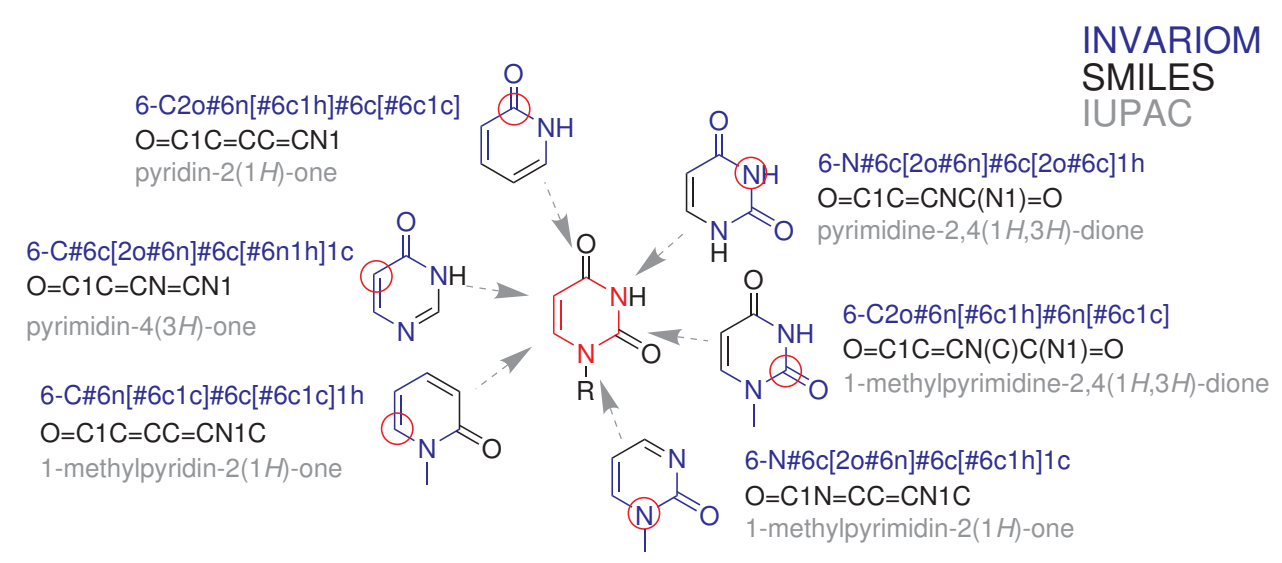

Abbildung 6.7: Modellverbindungen für Uridin, die im Invariom-Ansatz zur Rekonstruktion der molekularen Elektronendichte aus asphärischen Atomformfaktoren verwendet werden.

\subsubsection{Fluorochinolone}

Eine weitere heteroaromatische Substanzklasse, für die Einträge in der Invariom-Datenbank erzeugt wurden, sind die Fluorochinolon-Antibiotika. Die Modellsubstanzen ihrer Ringsysteme werden gemeinsam mit ihren Strukturen in Kapitel 8 besprochen. 
Kapitel 7

Das Makrolid-Antibiotikum Roxithromycin 


\subsection{Pharmakologische Wirkung}

Als Wirkort von Makrolid-Antibiotika wurde das bakterielle Ribosom identifiziert [86]. Bei der Interaktion mit Nukleotiden der Peptidyltransferase blockieren sie den Kanal, durch den die frischen Peptide zum Reaktionszentrum der Peptidyltransferase hindurch gefädelt werden müssen. Dadurch kommt die Proteinsynthese zum Erliegen und die Bakterien sterben ab. Aufgrund dieser biologischen Funktion ist die Bestimmung der Elektronendichteverteilung $\rho(\mathbf{r})$ von besonderem Interesse für Charakterisierung und Quantifizierung der Interaktionen am Wirkort. Da dem bakteriellen Ribosom eine Schlüsselrolle bei der Proteinsynthese in der Zelle zukommt, eignet es sich insbesondere als Zielmolekül für die Entwicklung neuer Wirkstoffe.

\subsection{Messung und Datenreduktion}

Tabelle 7.1: Kristallographische Daten von Roxithromycin

Aus der von Sigma Aldrich kommerziell bezogenen Probe konnte ein Einkristall ausgewählt werden, der

\begin{tabular}{|c|c|}
\hline Summenformel & $\mathrm{C}_{41} \mathrm{H}_{76} \mathrm{~N}_{2} \mathrm{O}_{15} \cdot 0,88 \mathrm{H}_{2} \mathrm{O}$ \\
\hline Molmasse $[\mathrm{g} / \mathrm{mol}]$ & 852,96 \\
\hline Kristallsystem & Orthorhombisch \\
\hline Raumgruppe, $Z$ & $\mathrm{P} 2{ }_{1} 2_{1} 2_{1}$ (Nr. 19), 4 \\
\hline \multicolumn{2}{|l|}{ Gitterkonstanten } \\
\hline$a$ [Å] & $11,624(2)$ \\
\hline$b$ [Å] & $16,748(3)$ \\
\hline$c$ [Å] & $24,018(5)$ \\
\hline$V\left[\AA^{3}\right]$ & $4675,8(15)$ \\
\hline Dichte (im Kristall) $\left[\mathrm{g} / \mathrm{cm}^{3}\right.$ ] & 1.215 \\
\hline Absorptionskoeffizient $\mu\left[\mathrm{mm}^{-1}\right]$ & 0.057 \\
\hline$F(000)$ & 1859.10 \\
\hline Kristallgröße [mm] & $0,5 \times 0,35 \times 0,3$ \\
\hline Temperatur [K] & 100 \\
\hline Wellenlänge, $\lambda[\AA ̊]$ & Synchrotron, 0,5600 \\
\hline $2 \theta_{\max }\left[{ }^{\circ}\right]$ & 76,96 \\
\hline $\sin \theta_{\max } / \lambda\left[\AA^{-1}\right]$ & 1,11 \\
\hline \multirow[t]{3}{*}{$h, k, l$ Bereich } & $-22 \Rightarrow h \Rightarrow 25$ \\
\hline & $-37 \Rightarrow k \Rightarrow 37$ \\
\hline & $-53 \Rightarrow l \Rightarrow 53$ \\
\hline Gemessene Reflexe & 474571 \\
\hline Symmetrieunabhängige Reflexe & 28461 \\
\hline Vollständigkeit & $99,6 \%$ \\
\hline Redundanz & 16,60 \\
\hline$R_{\text {int }}\left(F^{2}\right)$ & 0,0341 \\
\hline
\end{tabular}
für eine hochaufgelöste Messung geeignet war. Bei der Messung am Deutschen Elektronen-Synchrotron (DESY), Messplatz F1, wurde $\lambda=$ 0,5600 Å als Wellenlänge gewählt. Die Datensammlung erfolgte bei einem Detektorabstand von $67 \mathrm{~mm}$ durch Drehungen um die $\phi$-Achse. In zwei Durchläufen bei der Winkelstellung $2 \theta=0^{\circ}$ wurden mit einem Inkrement von $1^{\circ}$ in $\phi$ je 360 Einzelbilder gemessen. Die Belichtungszeit betrug dabei 8 und 12 Sekunden pro Bild. Bei zwei weiteren Durchläufen mit $2 \theta=-40^{\circ}$ wurden mit einem Inkrement von $1,5^{\circ}$ in $\phi$ je 240 Einzelbil- 
der gemessen. Dabei wurde 220 Sekunden pro Bild belichtet. Es wurde eine maximale Auflösung von $\sin \theta / \lambda=1,25 \AA^{-1}$ erreicht.

Der Datensatz wurden mit XDS [87] integriert und mit XPREP (Bruker AXS) skaliert und gemittelt. Die kristallographischen Daten sind in Tabelle 7.1 aufgeführt. Als Resultat einer Analyse der Daten mit den Kriterien $I(\vec{h}) / \sigma(\vec{h}), R_{\text {int }}$ und der Redundanz wurden die Daten bei $d=0,45 \AA$ bzw. $\sin \theta / \lambda=1,11 \AA^{-1}$ geschnitten. Die Auswertung des Datensatzes wurde im Jahr 2010 in der Zeitschrift Acta Crystallographica B publiziert [66].

\subsection{Strukturelle Eigenschaften}

Roxithromycin gehört zur Substanzklasse der Makrolid-Antibiotika deren charakteristisches chemisches Grundgerüst aus einem 14-gliedrigen Lacton-System besteht. Die Positionen 3 und 5 sind über glycosidische Bindungen mit Zuckern substituiert. $\alpha$-L-Cladinose befindet sich an Positionen 3 und $\beta$-D-Desosamin an Position 5. Position 9 ist als Oxim funktionalisiert [88]. Die Strukturformel von Roxithromycin ist in Abbildung 7.1 gezeigt. Atome, die in Wasserstoffbrückenbindungen involviert sind und Kohlenstoffatome, an die fehlgeordnete Wasserstoffato-

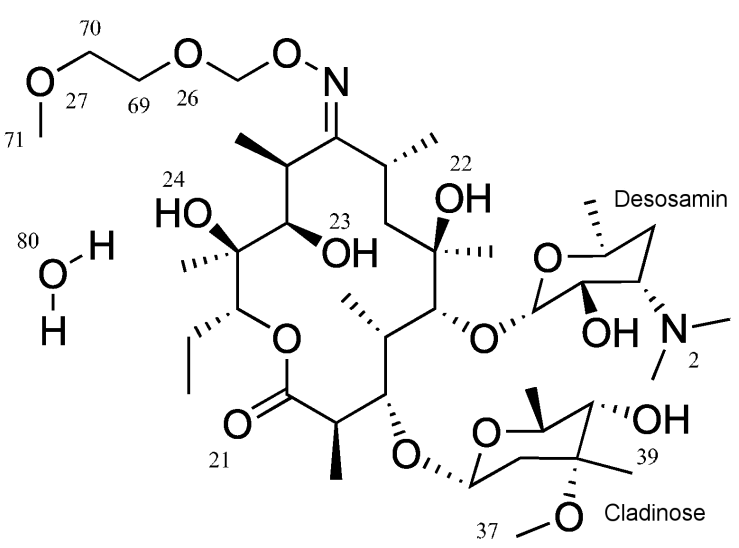

Abbildung 7.1: Strukturformel von Roxithromycin. Die Atomnummern sind für Atome gezeigt, welche in Wasserstoffbrückenbindungen involviert sind und für Kohlenstoffatome an die fehlgeordneten Wasserstoffatome gebunden sind. me gebunden sind, wurden nummeriert.

\subsection{Sphärische Verfeinerung}

Die Strukturlösung erfolgte mit direkten Methoden im Programm SHELXS. SHELXL wurde für die initiale Verfeinerung mit dem Modell unabhängiger Atome verwendet [43]. Dabei wurden die Koordinaten und Auslenkungsparameter (NichtWasserstoffatome anisotrop, Wasserstoffatome isotrop) aller Atome des Makro- 
lids frei verfeinert. Das kokristallisierte Wassermolekül war nicht vollständig populiert. Die Verfeinerung mit einer freien Variablen ergab eine Besetzung von 0,884(3). Die isotropen Auslenkungsparameter der beiden Wasserstoffatome (H80A und H80B) wurden dabei mit der Bedingung $U_{\text {eq }}(\mathrm{O})=1,5 \cdot U_{\text {iso }}(\mathrm{H})$ in Abhängigkeit des Auslenkungsparameters vom Sauerstoffatom (O80) verfeinert.

\subsubsection{Positionen von Wasserstoffatomen}

Die Kartierung der Restelektronendichte unter Aussparung eines Teils der Struktur wird häufig in der Proteinkristallographie angewendet. Dabei kann die Position von Liganden oder anderen kritischen Strukturfragmenten verifi-

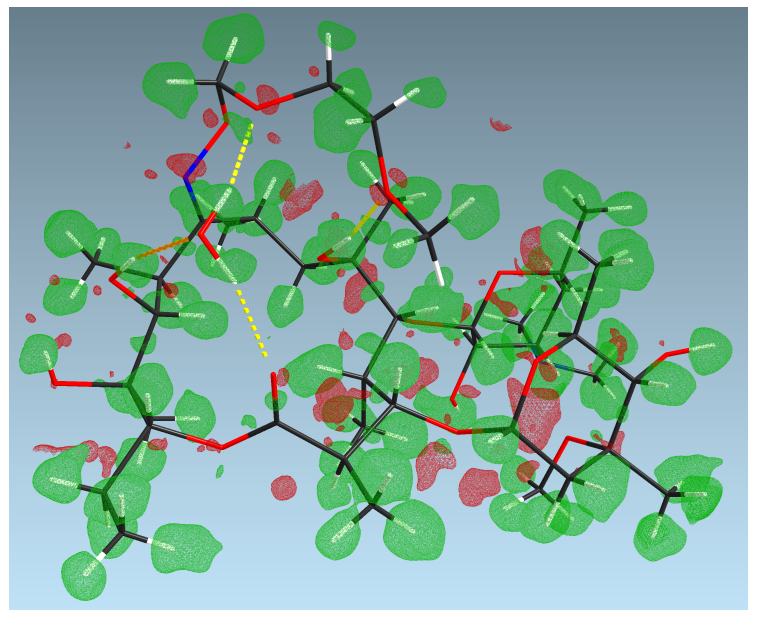

Abbildung 7.2: OMIT map von Roxithromycin. Die Restelektronendichte wurde unter Aussparung der Wasserstoffatome berechnet und ist mit SHELXLE [89] bei Werten von 0,2 und $-0,2 \mathrm{e} / \AA^{3}$ kartiert. ziert werden. Im Englischen spricht man dabei von einer sog. OMIT map. Während die Phasenwinkel $\Phi(\vec{h})$ dabei aus dem gesamten Modell stammen, werden die Strukturfaktoren $F_{c}(\vec{h})$ ohne die ausgelassenen Atome berechnet. Zur Verifizierung der Wasserstoffatompositionen wurde die Restelektronendichtekarte unter Aussparung der Wasserstoffatome berechnet. In Abbildung 7.2 ist die entsprechende OMIT map zu sehen, in der die Restelektronendichte als Gitter bei den Werten $\pm 0,2 \mathbf{e} / \AA^{3}$ eingezeichnet ist. Alle Wasserstoffatome sind von grünen Restelektronendichtegittern umgeben, was zeigt, dass die Position der Wasserstoffatome gesichert ist. Die experimentelle Beobachtung von Wasserstoffatomen ist aufgrund des geringen Streubeitrags nicht selbstverständlich.

\subsection{Absolute Struktur}

Die Struktur ist chiral und weist 18 stereogene Zentren auf. Mit der Wellenlänge von $\lambda=0,5600 \AA$ und Sauerstoff als schwerstem Atom ist das anormale 
Signal in den Daten nur gering. Durch die hohe Auflösung standen jedoch ausreichend Bijvoet-Differenzen [14] zur Verfügung, um eine verlässliche Aussage über die absolute Struktur treffen zu können. Während der Parameter nach Flack [13] mit 0,05(15) eine definitionsgemäs̈ zu hohe Standardabweichung hatte, ergab der Parameter mit dem gewichteten Verfahren nach Parsons [15] einen Wert von 0,08(4) und ermöglichte so die sichere Bestimmung der absolute Struktur im Rahmen der erlaubten Standardabweichung (10\%). An dieser Stelle wurde nur das Promolekülmodell verwendet.

\subsection{Invariom-Verfeinerung}

Ausgehend von der Molekülgeometrie aus der Verfeinerung mit sphärischen Atomformfaktoren wurde mit dem Programm InVARIOMTOOL [49] der InvariomTransfer durchgeführt. Für die anschließende Invariom-Verfeinerung [33] wurden die X-H-Abstände auf die Werte der quantenchemischen Geometrieoptimierungen gesetzt, welche eine genauere Unterscheidung der funktionellen Gruppen erlauben als Abstände aus Neutronenbeugungsexperimenten [30, 42]. Anschließend wurden die transferierten Multipol- und $\kappa$-Parameter des Multipolmodells [31] festgehalten und nur die Koordinaten und Auslenkungsparameter im Programm XD verfeinert [90]. Es wurde gegen $F(\vec{h})$ verfeinert und mit $w(\vec{h})=$ $1 / \sigma^{2}\left[F_{o}^{2}(\vec{h})\right]$ gewichtet. Mit dem Hirshfeld-Test wurde verifiziert, ob alle Auslenkungsparameter benachbarter Atome physikalisch sinnvolle Werte annehmen. Nur bei zwei Bindungen wurde der Hirshfeld-Test verletzt. Dabei betrugen die Werte für O27-C70 $1,6 \cdot 10^{-3} \AA^{2}$ und für O40-C37 $1,2 \cdot 10^{-3} \AA^{2}$. Sie liegen damit nur leicht oberhalb des Kriteriums von $1,0 \cdot 10^{-3} \AA^{2}$. Eine vollständige Liste des Hirshfeld-Tests befindet

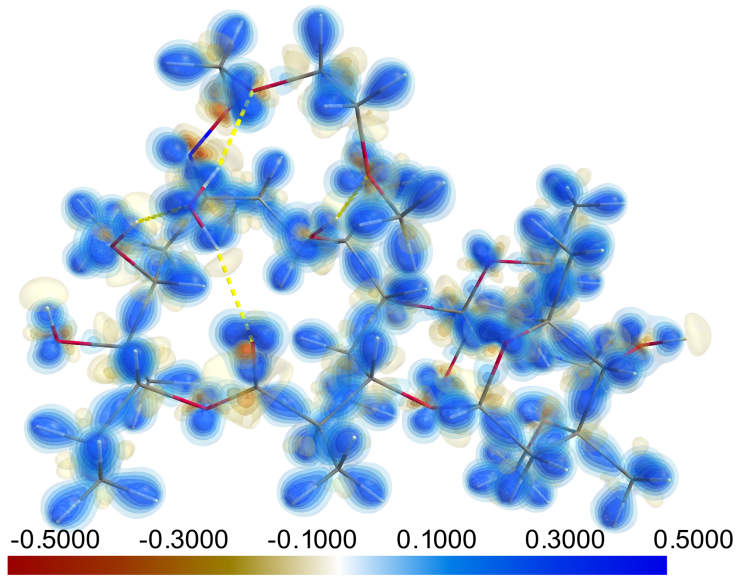

Abbildung 7.3: Deformationselektronendichte von Roxithromycin nach der Invariom-Verfeinerung. Die Isooberflächen wurden mit MOLECOOLQT [72] im Bereich $-0,5 \leq \mathbf{e} / \AA^{3} \leq 0,5$ bei einem Abstand von $0,1 \mathbf{e} / \AA^{3}$ eingezeichnet. sich im Anhang. Die Deformationselektronendichte wird in Abbildung 7.3 gezeigt. Dabei handelt es sich um den sphärischen Anteil der Elektronendichte. Da bei Roxithromycin nur maximal 
fünf Atome in einer Ebene sind, wurde die Deformationselektronendichte dreidimensional mit zehn Isooberflächen im Bereich $-0,5 \leq \mathbf{e} / \AA^{3} \leq 0,5$ bei einem Abstand von $0,1 \mathbf{e} / \AA^{3}$ gezeigt. Die Deformationselektronendichte repräsentiert auch die Verbesserung des Invariom-Modells gegenüber dem Modell unabhängiger Atome, welches keine Wasserstoffatome enthält.

\subsubsection{Anisotrope Auslenkungsparameter}

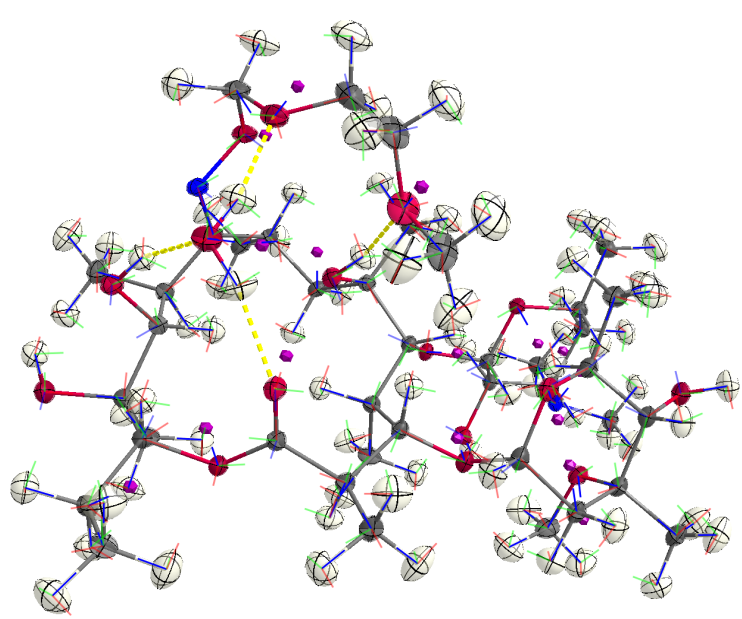

Abbildung 7.4: Anisotrope Auslenkungsparameter aller Atome mit 50\% Wahrscheinlichkeit und lokale Koordinatenachsen (x, y, z) von Roxithromycin.
Der SHADE2-Server [91, 92] wurde zur Berechnung anisotroper Auslenkungsparameter für Wasserstoffatome verwendet. Dabei wird der Auslenkungsparameter in externe und interne Moden unterteilt. Die externen Moden werden durch TLSNäherung (engl. Translation, Vibration, Screw) [93] mit Hilfe der Auslenkungsparameter von NichtWasserstoffatomen einer zu definierenden, rigiden Einheit des Moleküls bestimmt. Für die internen Moden werden Ergebnisse aus Neutronenbeugungsexperimenten verwendet. Die Moleküle in der asymmetrischen Einheit wurde in vier Gruppen unterteilt. Die beiden Zucker, der Makrolid-Zyklus und die Oxim-Kette wurden jeweils als rigide Körper definiert. Mit der anisotropen Beschreibung aller Atome wurde die InvariomVerfeinerung (IV) wiederholt. In Abbildung 7.4 werden die anisotropen Auslenkungsparameter aller Atome mit 50\% Wahrscheinlichkeit gezeigt. Zusätzlich sind die lokalen Koordinatenachsen $\mathrm{x}, \mathrm{y}, \mathrm{z}$ im Farbcode rot/grün/blau (rgb) gezeigt. Um die maximale lokale Symmetrie beschreiben zu können, wurden dafür 15 Hilfsatome (engl. dummy) berechnet, die zur Orientierung der lokalen Koordinatenachsen verwendet werden und mit MoLECOOLQT [72] als rosafarbene Ikosaeder eingezeichnet sind. 


\subsection{Multipolverfeinerung}

Die experimentelle Multipolverfeinerung wurde ausgehend von den InvariomVerfeinerungen durchgeführt. Um mit der Invariom-Verfeinerung vergleichbar zu sein wurden in dem EMV keine Atompositionen verfeinert. Die lokale Symmetrie und chemische Gleichheit (engl. chemical constraints) wurde mit INVARIOMTOOL [49] entsprechend der Invariom-Namen eingeführt. Das Startmodell aus $x$ d.mas.HasSameSymm wurde nur geringfügig verändert: Für Atome, die Wasserstoffbrückenbindungen eingehen (O23, O24, O26, O27, O59, O39 und ggf. kovalent gebundenen Wasserstoffatome) wurde die chemische Gleichheit entfernt und die Multipolparameter unabhängig von anderen Atomen verfeinert. Die Multipolexpansion betrug $l_{\max }=4$ für alle Nicht-Wasserstoffatome. Die Elektronendichtebeschreibung von Wasserstoffatomen wurde mit hybriden Streufaktoren realisiert. Multipolpopulationen $\left(l_{\max } \geq 2\right)$ wurden dabei aus der Datenbank transferiert und festgehalten, während Monopol- und Dipolpopulationen verfeinert wurden. Bei Wasserstoffatomen in Wasserstoffbrückenbindungen wurden auch Guadropole verfeinert. Da Wasserstoffatome keine Kernelektronen aufweisen ist der Kontraktionsparameter $\kappa$ in der Verfeinerung stark mit dem Auslenkungsparameter korreliert und konnte hier nicht verfeinert werden. Statt dessen wurden die $\kappa$-Parameter aus der InvariomDatenbank beibehalten. Diese Verfeinerung wird im folgenden als experimentelle Multipolverfeinerung (EMV) bezeichnet.

\subsection{Hybride Streufaktoren}

Für die Einträge der Invariom-Datenbank von Wasserstoffatomen werden alle bindungsgerichteten Multipolparameter (Monopol, Dipol, Guadropol, Octopol und Hexadecapol) gegen die theoretischen Daten verfeinert (siehe Abschnitt 5.4.3 auf Seite 45). Da gegen die experimentellen Daten zwar Monopole und Dipole sowie bei Wasserstoffbrückenbindungen Guadropole verfeinert, die höheren Pole (ggf. Quadropole, Octopole und Hexadecapole) nach dem InvariomTransfer jedoch festgehalten werden, resultieren daraus hybride Streufaktoren für Wasserstoffatome. Um den Einfluss dieser hybriden Streufaktoren auf die Elektronendichte zu untersuchen, wurde eine Differenzelektronendichte aus der Multipolverfeinerung EMV und einer klassischen Multipolverfeinerung (KMV), ohne Werte von Guadropolen, Octopolen und Hexadecapolen aus der Invariom-Datenbank, verglichen. Dabei waren sowohl Atompositionen als 


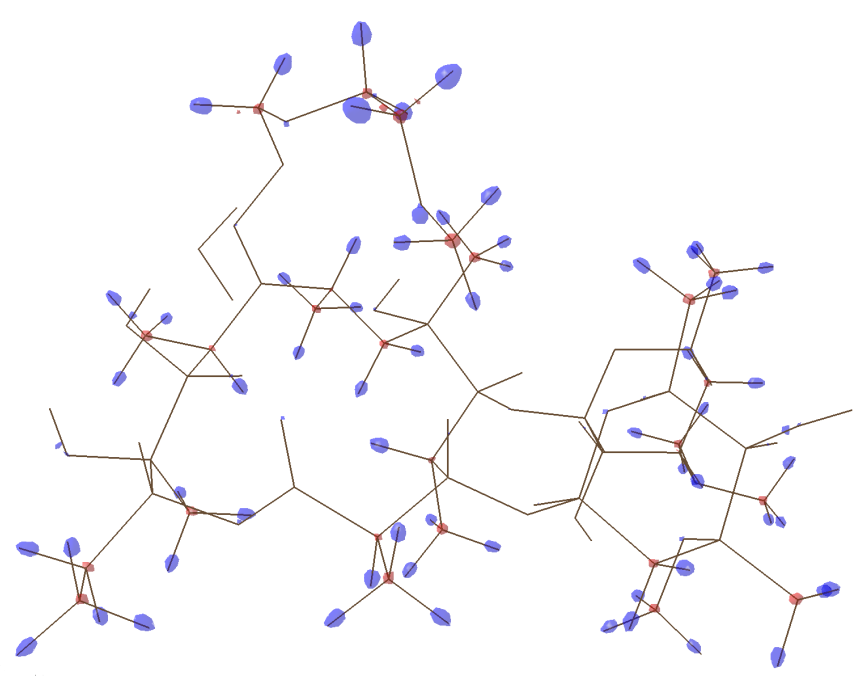

Abbildung 7.5: Hybride Streufaktoren von Wasserstoffatomen mit theoretischen Werten für Quadropole, Octopole und Hexadecapole im Vergleich zu klassischen Streufaktoren. Die Differenzelektronendichte aus EMV und KMV ist als Isooberfläche bei Werten von $\mathbf{0 , 0 7 5}$ und $-0,075$ e/ $\AA^{3}$ kartiert.

auch $\kappa$-Parameter identisch. Die Elektronendichte unterscheidet sich also nur durch die höheren Multipole der Wasserstoffatome. Die Differenzelektronendichte aus EMV und KMV ist als Isooberfläche bei Werten von $\pm 0,075 \mathbf{e} / \AA^{3}$ in Abbildung 7.5 kartiert und zeigt eine systematische Verschiebung der Elektronendichte vom Monopol der Kohlenstoffatome zu den jeweils gebundenen Wasserstoffatomen. Bei $\mathrm{R}_{2} \mathrm{CH}_{2}$ und $\mathrm{RCH}_{3}$ Gruppen ist dieser Effekt besonders ausgeprägt. Da die Limitierung von $l_{\text {max }}$ bei Wasserstoffatomen aus der begrenzten Streukraft resultiert, ist die Einbeziehung zusätzlicher Information über den Streubeitrag des Wasserstoffatoms eine sinnvolle Ergänzung, um die Verlässlichkeit der Aussagen von Multipolverfeinerungen zu erhöhen und gleichzeitig die Übereinstimmung mit theoretischen Rechnungen zu verbessern. Der Vergleich hybrider Streufaktoren mit der klassischen Multipolverfeinerung wurde erstmals am Datensatz von Roxithromycin untersucht und publiziert [66].

\subsection{Validierung und Modellkorrektur}

\subsubsection{Differenzelektronendichte}

Im experimentelle Multipolmodell wurde die Rotationsfehlordnung der Wasserstoffatome an C71, dem terminalen Kohlenstoff der Oxim-Kette, von den 


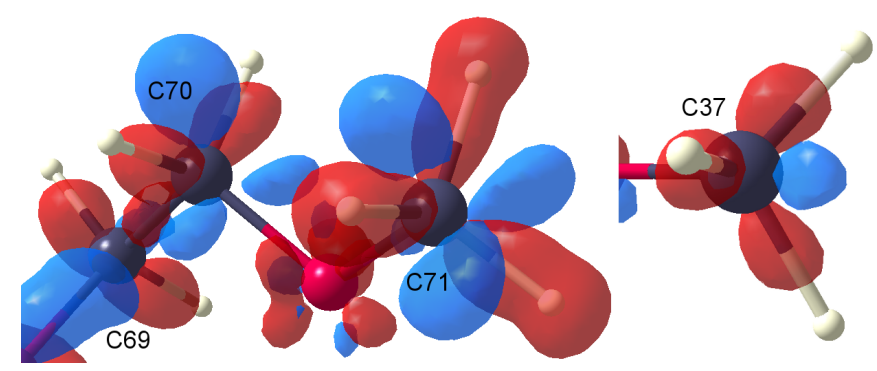

Abbildung 7.6: Differenzelektronendichte aus Multipolverfeinerung (EMV) und Invariom-Verfeinerung (IV). Links: Die terminalen Atome der Oxime Kette (C69, C70 und C71). Rechts: Atom C37 des Cladinose Zuckers. Die Isooberflächen sind bei 0,1 und $-0,1 \mathrm{e} / \AA^{3}$ kartiert.

Multipolparameter kompensiert. Im theoriebasierten Invariom-Modell tritt dieser Effekt nicht auf. In beiden Verfeinerungen wurde nur eine Komponente der Fehlordnung verfeinert, da eine Verfeinerung mit zwei diskreten Positionen nur eine geringe Verbesserung der Gütefaktoren (FOM) ergab. Aus der Differenzelektronendichte zwischen dem Invariom-Modell und der experimentellen Multipolverfeinerung lässt sich der Unterschied deutlich herausstellen. Diese sind als Isooberflächen bei $\pm 0,1 \mathbf{e} / \AA^{3}$ in Abbildung 7.6 gezeigt. Die rote Isooberfläche zeigt dabei Regionen, in denen bei der Multipolverfeinerung weniger Dichte als bei der Invariom-Verfeinerung vorhanden ist. Bei der blauen Isooberfläche ist es umgekehrt. Besonders an von C71 der Methoxygruppe sieht man gut, dass auf Bindungen zu Wasserstoffatomen weniger Dichte und $60^{\circ}$ dazu versetzt mehr Elektronendichte aus der experimentellen Multipolverfeinerung resultiert als aus der Invariom-Verfeinerung.

\subsubsection{Deformationselektronendichte}

In Abbildung 7.7 wird gezeigt, dass die Rotationsfehlordnung zwischen den Positionen der Wasserstoffatome in Form eines Torus verteilt ist. Eine Verfeinerung von geteilte Positionen eignet sich jedoch nur in Fällen, bei denen zwei oder mehr diskrete Atompositionen im Kristall vorhanden sind. Die kontinuierliche Verteilung der Elektronendichte macht deutlich, warum eine klassische Verfeinerung mit geteilten Positionen hier nicht zu einer Modellverbesserung geführt hat. Zusätzlich ist der Anteil der Fehlordnung an der Gesamtelektronendichte gering. 


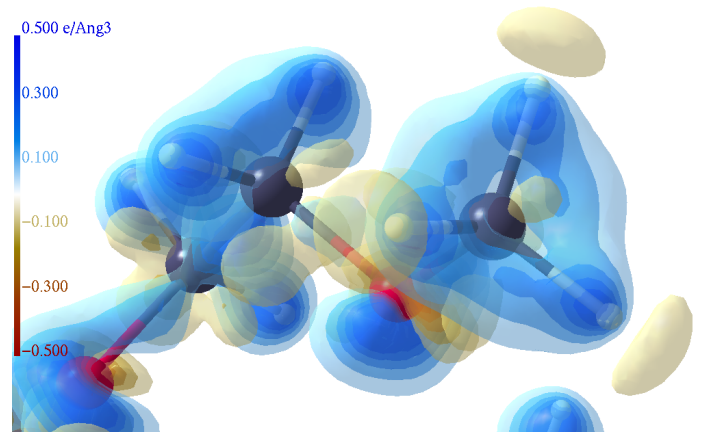

Abbildung 7.7: Deformationselektronendichte von Roxithromycin nach der Multipolverfeinerung. Die Isooberflächen wurden im Bereich $-0,5 \leq$ $\mathbf{e} / \AA^{3} \leq 0,5$ im Abstand von $0,1 \mathbf{e} / \AA^{3}$ eingezeichnet.

\subsubsection{Gemischte Multipolverfeinerung (GMV)}

Da die Modellierung mit geteilten Positionen nicht erfolgreich war, wurde ohne geteilte Positionen fortgefahren. Das Verfeinerungsmodell wurde so angepasst, dass in den fehlgeordneten Bereichen keine Multipolparameter der Atome verfeinert wurden, sondern diese auf den Werten der Invariom-Verfeinerung festgehalten wurden. Es handelt sich also um eine Mischform zwischen einer Invariom- und einer experimentellen Multipolverfeinerung und wurde deshalb als gemischte Multipolverfeinerung (GMV) bezeichnet. Im Gegensatz zum EMV, wo die Geometrie aus der Invariom-Verfeinerung beibehalten wurde, sind im GMV auch Positionsparameter aller Nicht-Wasserstoffatome verfeinert. Die Verfeinerungsergebnisse sind für alle drei Modelle in Tabelle 7.2 angegeben.

\begin{tabular}{lrrr} 
FOM & IV & EMV & GMV \\
\hline Parameter & 532 & 725 & 850 \\
Reflexe & 26324 & 26324 & 26324 \\
$R(F)[\%]$ & 2,21 & 2,04 & 2,01 \\
$R_{w}(F)[\%]$ & 1,90 & 1,67 & 1,68 \\
$R_{\text {all }}(F)[\%]$ & 2,46 & 2,16 & 2,26 \\
$S$ Goof & 4,03 & 3,54 & 3,59 \\
$\Delta \rho_{\min }\left[e / \AA^{3}\right]$ & $-0,30$ & $-0,33$ & $-0,20$ \\
$\Delta \rho_{\max }\left[e / \AA^{3}\right]$ & 0,33 & 0,32 & 0,20 \\
\hline
\end{tabular}

Tabelle 7.2: Gütefaktoren (FOM) der Invariom-Verfeinerung (IV), der experimentellen Multipolverfeinerung (EMV) und der gemischten Multipolverfeinerung (GMV) 


\subsubsection{Guantifizierung der Fehlordnung}

Die quantitative Charakterisierung der Fehlordnung wird durch die atomaren Eigenschaften nach Baders QTAIM (engl. quantum theory of atoms in molecules) [94] ermöglicht. Die atomaren Ladungen und Volumina wurde mit XDPROP berechnet und sind in Tabelle 7.3 aufgeführt. Aus den integrierten Eigenschaften geht hervor, dass C71 der Methoxygruppe von sehr stark betroffen ist. In der GMV hat C71 eine positive Ladung von 0,28e, wie man es bei einem Kohlenstoffatom einer Methoxygruppe erwarten würde. Bei einer Ladung des C71 von 0,01e in der EMV wird die Ladung von den gebundenen und fehlgeordneten Wasserstoffatomen kompensiert, die je eine Ladung von $0,23 e$ besitzen. In der GMV beträgt die Ladung der an die Methoxygruppe gebundenen Wasserstoffatome hingegen nur 0,08e. Es zeichnet sich hier also ein genereller Trend ab. Das Kohlenstoffatom der EMV hat ein größeres Volumen, in welchem sich auch eine größere Zahl an Elektronen befindet. Entsprechend haben die Wasserstoffatome ein kleineres Volumen und auch eine geringere Anzahl an Elektronen.

\begin{tabular}{l|ccc|ccc} 
& \multicolumn{3}{|c|}{ Ladung $\mathbf{G}$} & \multicolumn{3}{r}{$\mathbf{V}_{\text {tot }}$} \\
Atom & IV & EMV & GMv & IV & EMv & GMV \\
\hline C37 & 0,26 & 0,11 & 0,28 & 10,3 & 11,7 & 10,6 \\
H37A & 0,08 & 0,08 & 0,08 & 7,9 & 7,4 & 7,7 \\
H37B & 0,08 & 0,07 & 0,08 & 9,3 & 8,7 & 9,1 \\
H37C & 0,08 & 0,08 & 0,08 & 8,9 & 8,2 & 8,6 \\
\hline C69 & 0,28 & 0,21 & 0,29 & 8,6 & 9,5 & 8,8 \\
H69A & 0,06 & 0,10 & 0,06 & 10,3 & 9,7 & 10,4 \\
H69B & 0,05 & 0,09 & 0,06 & 9,1 & 8,3 & 8,9 \\
\hline C70 & 0,31 & 0,23 & 0,31 & 8,5 & 10,1 & 8,7 \\
H70A & 0,06 & 0,12 & 0,07 & 7,3 & 6,4 & 7,0 \\
H70B & 0,05 & 0,11 & 0,06 & 7,6 & 6,9 & 7,3 \\
\hline C71 & 0,25 & 0,01 & 0,26 & 11,8 & 15,5 & 12,6 \\
H71A & 0,07 & 0,23 & 0,07 & 8,3 & 6,1 & 7,9 \\
H71B & 0,08 & 0,23 & 0,08 & 7,9 & 6,2 & 7,7 \\
H71C & 0,09 & 0,23 & 0,09 & 8,2 & 6,2 & 7,8 \\
\hline
\end{tabular}

Tabelle 7.3: Atomare Ladungen und Volumina der von Rotationsfehlordnung betroffenen Gruppen aus den drei verschiedenen Verfeinerungen Roxithromycins.

Aufgrund der Zeitauflösung ist im Röntgenbeugungsexperiment keine Unterscheidung zwischen statischer und dynamischer Fehlordnung möglich. Diese Begriffe sind deshalb in Bezug auf experimentelle Ergebnisse nicht zweckdienlich und ähnlich problematisch wie die Verwendung des Begriffs Temperaturparameter anstelle von Auslenkungsparameter. Um diese uneindeutigen Begrifflichkeit zu vermeiden, wird deshalb eine Einteilung von Fehlordnungen 
in die Kategorien diskret und kontinuierlich vorgeschlagen. Diese Unterscheidung wurde bereits in der Monographie „Crystal Structure Refinement“ [95] zur Charakterisierung von Fehlordnungen eingeführt. Die genauere physikalische Natur kann durch externe Informationen wie theoretische Berechnungen ergründet werde. So legen Moleküldynamik-Simulationen in einem anderen Fall nahe, dass es sich bei der kontinuierlichen Rotationsfehlordnung um ein dynamisches Phänomen handelt [83]. Die ungewollte Beschreibung kontinuierlicher (dynamischer) Fehlordnung durch Multipolparameter konnte auch bei Untersuchungen an der Struktur von Paracetamol als mögliche Fehlerquelle einer nicht validierten experimentellen Multipolverfeinerung identifiziert werden [96]. Die Flexibilität der Multipolparameter eine Rotationsfehlordnung abzubilden ist bereits in den genannten Publikationen diskutiert worden [83, 96, 66], bedarf aber noch weiterer Untersuchungen.

\subsubsection{Restelektronendichte}

Die Restelektronendichte wird üblicher Weise wie in Tabelle 7.2 als $\Delta \rho_{\max }$ und $\Delta \rho_{\min }$ angegeben. Daraus geht jedoch nicht die dreidimensionale Verteilung der Restelektronendichte in der asymmetrischen Einheit hervor. Seit Juni 2011 kann im Programm MolecoolQT [72] die Restelektronendichte durch Einlesen der XD-Datei $x d$.fou dreidimensional dargestellt werden. Diese besonders hilfreiche Form der Validierung ist in Abbildung 7.8 für die sphärische Verfeinerung (a), die Invariom-Verfeinerung (b), die gemischte Multipolverfeinerung (c) gezeigt. Die Restdichtekarte $\left(F_{o}-F_{c}\right)$ ist im sphärischen Fall bei $\pm 0,1$ e/ $\AA^{3}$ in allen anderen Fällen bei $\pm 0,05$ e/ $\AA^{3}$ als Gitter gezeigt. Nach der GMV ist die Restelektronendichte mit Ausnahme der Region von Sauerstoffatom O27 gleichmäßig verteilt. Um die Atomposition von $\mathrm{O} 27$ weist sie jedoch ein charakteristisches Schema in Form von „+ - +-“ auf, welches vermuten lässt, dass die harmonische Näherung der Aufenthaltswahrscheinlichkeitsverteilung für Atom O27 nicht ausreichend ist [97].

\subsubsection{Anharmonische Schwingung}

Durch Verfeinerung von Gram-Charlier-Parametern dritter Ordnung für O27 verbessert sich die Restelektronendichte und der R(F). Sowohl als Block-Matrix als auch bei gemeinsamer Verfeinerung mit allen anderen Parametern wird nach 13 Zyklen eine Konvergenz von $10^{-5}$ A erreicht. Die Restelektronendichte dieser anharmonischen Verfeinerung und eine zusätzliche Differenzelektro- 

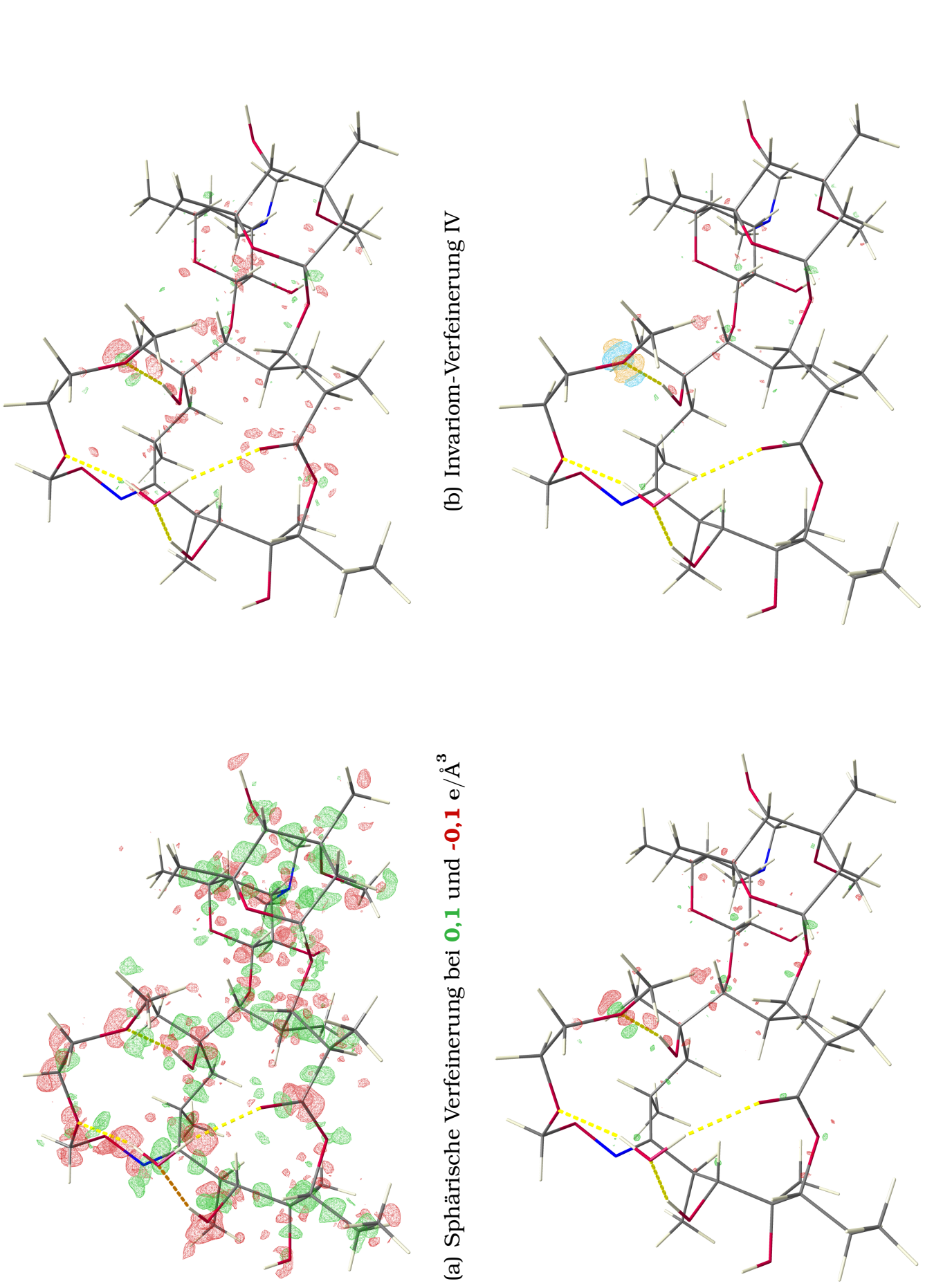

草兽泀

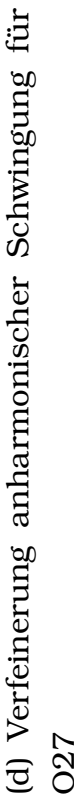

$\vec{\nabla}$

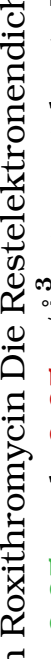

نे

है

$\infty_{0}$

की

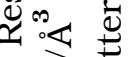

过

공 응

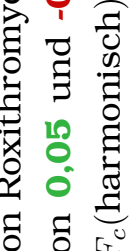

कृ

造

롱

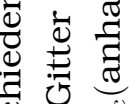

可

药

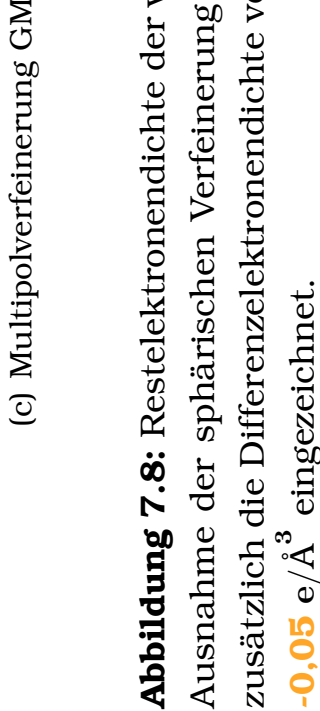


nendichte von $F_{c}$ (anharmonisch) $-F_{c}$ (harmonisch) sind bei Werten von $\pm 0,05$ e/ $\AA^{3}$ in Abbildung 7.8 (d) gezeigt. Aus dem Vergleich der Abbildungen 7.8 (c) und (d) geht hervor, dass die Verfeinerung anharmonischer Schwingung eine sinnvolle Modellverbesserung darstellt und die größten Charakteristika der Restelektronendichtekarte auf diese Weise erfolgreich beschrieben werden. Ein Zusammenhang der anharmonischen Schwingung von Atom O27 mit der Rotationsfehlordnung der Wasserstoffatome von C71 ist plausibel. Die Verfeinerung von Gram-Charlier-Parametern bei zukünftigen Untersuchungen ist deshalb besonders im Hinblick auf eine mögliche Korrelation von anharmonischer Schwingung mit der Rotationsfehlordnung benachbarter Gruppe interessant. Die Auswirkungen anharmonischer Schwingungen wurden erst kürzlich diskutiert. [97]

\subsection{Wasserstoffbrücken}

\begin{tabular}{|c|c|c|c|c|c|c|c|}
\hline $\mathrm{D}-\mathrm{H} \cdots \mathrm{A}$ & D-H & $\mathrm{H} \cdots \mathrm{A}$ & $\mathrm{D} \cdots \mathrm{A}$ & $\mathrm{D}-\mathrm{H} \cdots \mathrm{A}$ & $\rho\left(\mathbf{r}_{\mathrm{BKP}}\right)$ & $\nabla^{2} \rho\left(\mathbf{r}_{\mathrm{BKP}}\right)$ & $\epsilon$ \\
\hline $\mathrm{O} 22-\mathrm{H} 22 \cdots \mathrm{O} 27$ & 0,96 & 1,89 & 2,83 & 163 & 0,19 & 2,2 & 0,07 \\
\hline $\mathrm{O} 23-\mathrm{H} 23 \cdots \mathrm{O} 80$ & 0,97 & 1,81 & 2,73 & 158 & 0,21 & 2,5 & 0,11 \\
\hline O80-H80A $\cdots$ O26 & 0,96 & 1,89 & 2,84 & 168 & 0,27 & 1,0 & 0,02 \\
\hline O80-H80B $\cdots$ O 21 & 0,96 & 1,96 & 2,91 & 167 & 0,16 & 1,2 & 0,05 \\
\hline O39-H39 $\cdots \mathrm{N}^{i}$ & 0,96 & 1,77 & 2,72 & 173 & 0,38 & 2,0 & 0,01 \\
\hline O59-H59... O39ii & 0,96 & 2,00 & 2,94 & 164 & 0,11 & 2,3 & 0,18 \\
\hline
\end{tabular}

Tabelle 7.4: Topologie der bindungskritischen Punkte von Wasserstoffbrückenbindungen in Roxithromycin der GMV. Die interatomaren Abstände sind in $\AA$ und Winkel in ${ }^{\circ}$ angegeben. Die Elektronendichte am bindungskritischen Punkt $\rho\left(\mathbf{r}_{\mathrm{BKP}}\right)$ ist in $e / \AA^{3}$ und die Laplacefunktion $\nabla^{2} \rho\left(\mathbf{r}_{\mathrm{BKP}}\right)$ in $e / \AA^{5}$ angegeben. $\epsilon$ ist die Elliptizität der Bindung.

Die Atome des Makrolids bilden eine intramolekulare Wasserstoffbrückenbindung und zwei intermolekulare Wasserstoffbrückenbindungen zu benachbarten Makroliden aus. Zusätzlich fungiert das kokristallisierte Wassermolekül im Fall von O23-H als Akzeptor und verbrückend zwischen dem Ether-Sauerstoff O26 und dem Carbonyl-Sauerstoff O21 als Donor. Geometrische Details der Wasserstoffbrücken sind in Tabelle $7.4 \mathrm{zu}$ finden. Eine topologische Analyse der bindungskritischen Punkte wurde ebenfalls durchgeführt. Dabei wurden für die Elektronendichte am bindungskritischen Punkt $\rho\left(\mathbf{r}_{\mathrm{BKP}}\right)$ der Verfeinerungen EMV und GMV sehr ähnliche Werte erhalten. Den grössten Unter- 
schied wies mit 0,03 e/ $\AA^{3}$ die Wasserstoffbücke H80A... O26 auf. Die Elektronendichte am BKP der GMV war für die Wasserstoffbrücke O39-H39 $\cdots \mathrm{N} 2^{i}$ mit $\rho\left(\mathbf{r}_{\mathrm{BKP}}\right)=0,38 \mathbf{e} / \AA^{3}$ am höchsten. Der Wert ist außergewöhnlich hoch, wird aber durch die zusätzliche Akzeptorfunktion von O39 in der O59-H59... O39 ${ }^{i i}$ Wasserstoffbrücke hervorgerufen. Der H23 ‥ O80-Kontakt weist bei sehr ähnlichen interatomaren Abständen nur einen Wert von $\rho\left(\mathbf{r}_{\mathrm{BKP}}\right)=0,21 \mathbf{e} / \AA^{3}$ auf.

\begin{tabular}{l|ccc|ccc}
$\mathrm{H} \cdots \mathrm{A}$ & IV & EMV & GMV & $G\left(\mathbf{r}_{\mathrm{BKP}}\right)$ & $V\left(\mathbf{r}_{\mathrm{BKP}}\right)$ & $E_{H B}[\mathrm{~kJ} / \mathrm{mol}]$ \\
\hline $\mathrm{H} 22 \cdots \mathrm{O} 27$ & 0,16 & 0,20 & 0,19 & 59,6 & $-59,3$ & $-29,6$ \\
$\mathrm{H} 23 \cdots \mathrm{O} 80$ & 0,22 & 0,21 & 0,21 & 68,6 & $-69,1$ & $-34,6$ \\
$\mathrm{H} 80 \mathrm{~A} \cdots \mathrm{O} 26$ & 0,18 & 0,24 & 0,27 & 53,4 & $-79,6$ & $-39,8$ \\
$\mathrm{H} 80 \mathrm{~B} \cdots \mathrm{O} 21$ & 0,14 & 0,15 & 0,16 & 36,5 & $-40,4$ & $-20,2$ \\
$\mathrm{H} 39 \cdots \mathrm{N}^{i}$ & 0,27 & 0,39 & 0,38 & 98,7 & $-142,9$ & $-71,4$ \\
$\mathrm{H} 59 \cdots \mathrm{O} 39{ }^{i i}$ & 0,13 & 0,11 & 0,11 & 49,7 & $-36,7$ & $-18,3$ \\
\hline \multicolumn{7}{l}{$\left(1-\mathrm{x}, \frac{1}{2}+\mathrm{y}, \frac{1}{2}-\mathrm{z}\right),{ }^{i i}\left(1-\mathrm{x}, \frac{1}{2}+\mathrm{y}, \frac{1}{2}-\mathrm{z}\right)$}
\end{tabular}

Tabelle 7.5: Zusammenhang zwischen der Elektronendichte am bindungskritischen Punkt $\rho\left(\mathbf{r}_{\mathrm{BKP}}\right)$, der kinetischen Energiedichte $G\left(\mathbf{r}_{\mathrm{BKP}}\right)$, der Potentiellen Energiedichte $V\left(\mathbf{r}_{\mathrm{BKP}}\right)$ und der Wasserstoffbrückenbindungsenergie $E_{H B}$ des GMV in $\mathrm{kJ} / \mathrm{mol}$

Zusätzlich wurde die Elektronendichte und die Laplacefunktion der bindungskritischen Punkte der GMV zur Berechnung von energetischen Eigenschaften verwendet. Die kinetische Energiedichte $G\left(\mathbf{r}_{\mathrm{BKP}}\right)$ nach Abramov et al. [67], die potentielle Energiedichte $V\left(\mathbf{r}_{\mathrm{BKP}}\right)$ und die Wasserstoffbrückenbindungsenergie $E_{H B}$ nach Espinosa et. al [68] wurden dabei entsprechend der empirischen Formeln berechnet und sind in Tabelle $7.5 \mathrm{zu}$ finden. Hier ergeben sich Energien im Bereich von 20 bis $40 \mathrm{~kJ} / \mathrm{mol}$. Die Topologie der Wasserstoffbrücke O39H39...N2 sorgt, bedingt durch die hohe Elektronendichte am bindungskritischen Punkt, für eine unrealistisch hohe Wasserstoffbrückenbindungsenergie von über $70 \mathrm{~kJ} / \mathrm{mol}$.

\subsection{Elektrostatisches Potential}

\subsubsection{Einfluss der Verfeinerung}

Wenn Rotationsfehlordnung, wie im Fall der EMV in den Multipolen kompensiert wird, hat dies einen starken Einfluss auf die elektrostatischen Eigenschaften. Werden diese Feinheiten der Modellierung nicht beachtet, ergeben 
sich Artefakte der molekularen Eigenschaften. Deswegen sollte geprüft werden, dass keine derartigen Effekte in das Elektronendichtemodell einfließen. In Abbildung 7.9 sind die elektrostatischen Potentiale vergleichend für die beiden Verfeinerungen EMV und IV gezeigt. Das elektrostatische Potential (ESP) wird dort als Farbgradient auf die molekulare Isooberfläche bei einem Wert von 0,0067 e/ $\AA^{3}$ abgebildet. Dabei handelt es sich um die Van-der-WaalsOberfläche, welche umgerechnet in atomare Einheiten 0,001 au beträgt. Die Zuordnung von Potentialwerten zur Farbkodierung ist in beiden Fällen gleich gewählt und in der Legende am Bildrand aufgeführt. Bei der experimentellen Multipolverfeinerung (EMV) weist die Oberfläche in blauer Farbe eine stark positives Potential von 0,2 e/Å auf, während sie in der Invariom-Verfeinerung nur ein leicht positives Potential von $0,04 \mathrm{e} / \AA ̊$ hat.

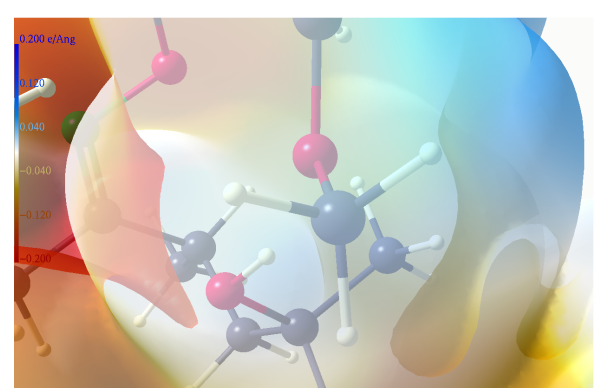

(a) Invariom-Verfeinerung (IV)

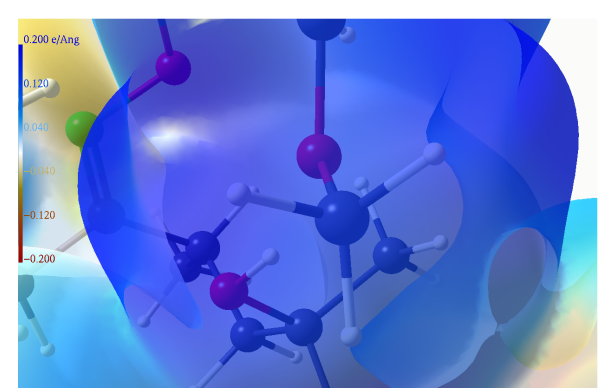

(b) Experimentelle Multipolverfeinerung (EMV)

Abbildung 7.9: Elektrostatisches Potential der Methoxygruppe von C71 kartiert als Farbcode auf der Van-der-Waals-Oberfläche.

\subsubsection{Elektrostatisches Potential der molekularen Oberfläche}

Das elektrostatische Potential wurde für die IV und die GMV für das gesamte Molekül berechnet, wobei das Potential des Wasssermoleküls nicht in die Berechnung einfloss. Die Potentiale werden in Abbildung 7.10 gezeigt. Auch wenn die beiden Isooberflächen und Potentiale qualitativ übereinstimmen, gibt es besonders an den funktionellen Gruppen, die in Wasserstoffbrückenbindungen involviert sind, deutliche Unterschiede. Die Dimethylamingruppe weist ein leicht negatives Potential im GMV auf, während sie in der IV eine neutrale oder leicht negative Ladung hat. 

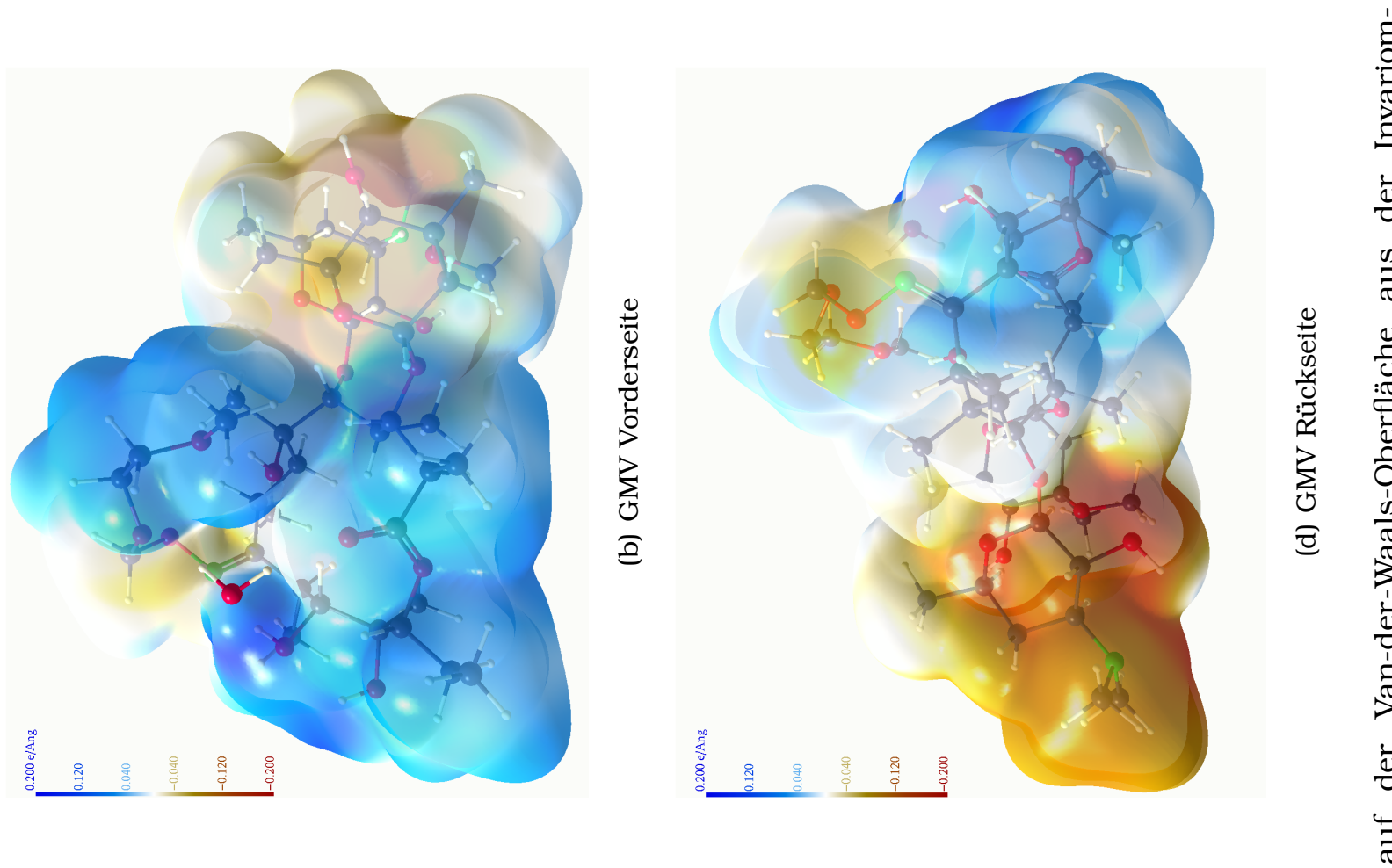

ᄅี
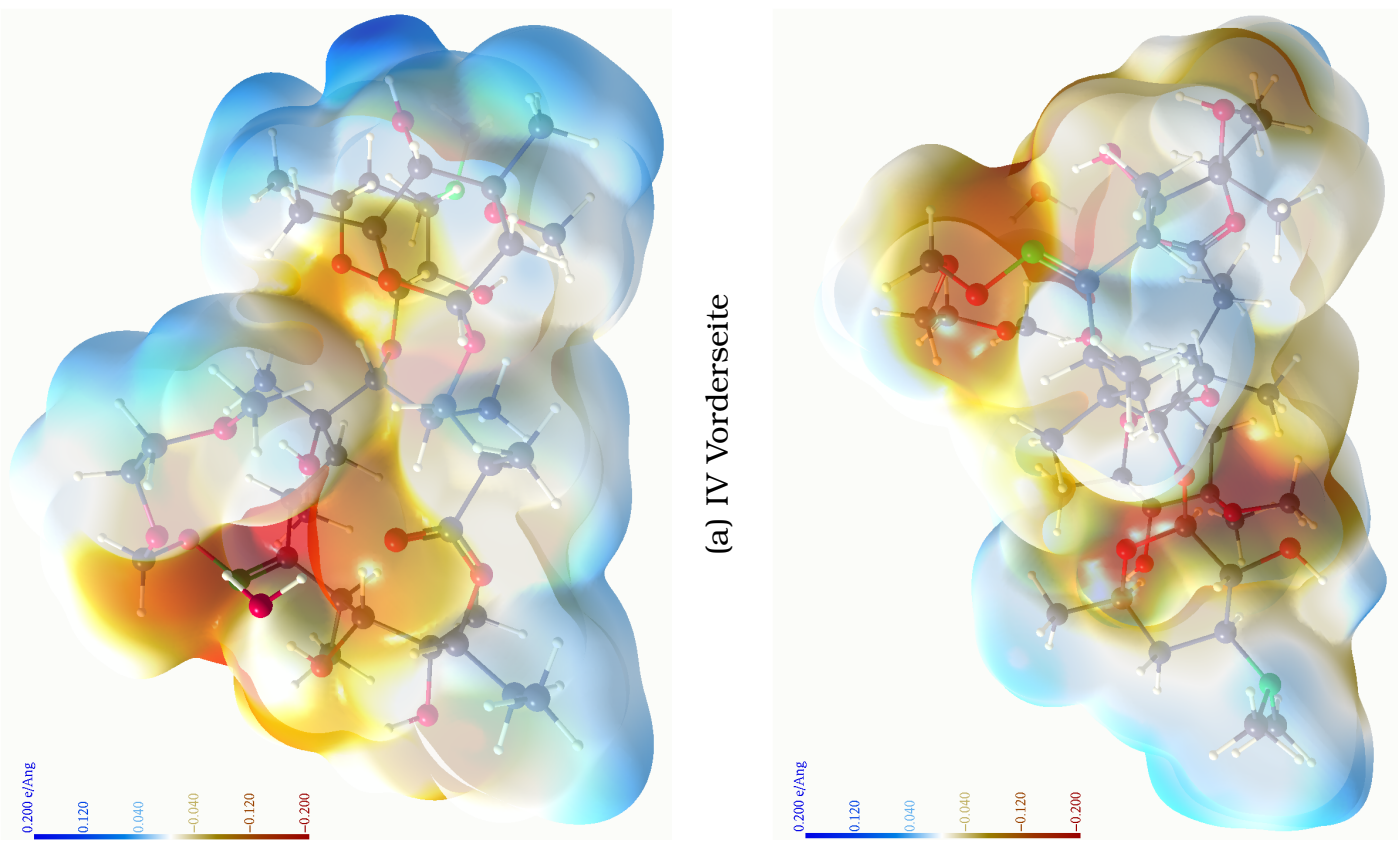

ष्ष

శ)

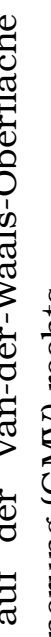

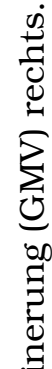

더

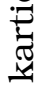

幽

졸

:

仓

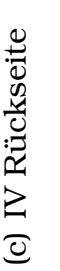

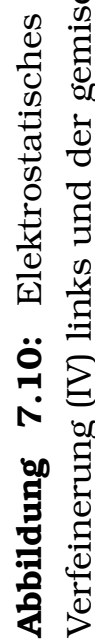




\section{Politzer-Analyse}

Neben der optischen Analyse der Oberflächenpotentiale kann auch das Verfahren nach Politzer et al. [63] verwendet werden. Dabei werden Durchschnittswerte vom positiven Potential $\bar{V}_{S}^{+}$, negativen Potential $\bar{V}_{S}^{-}$und vom Gesamtpotential $\bar{V}_{S}$ gebildet. Wie in Abschnitt 3.6.1 auf Seite 32 beschrieben wird zusätzlich die Varianz $\sigma_{\text {tot }}^{2}$ und Standardabweichung des Gesamtpotentials $\Pi$ berechnet. Die Ergebnisse für die drei Verfeinerungen (IV, EMV und GMV) wurden in Tabelle 7.6 zusammengefasst. Daraus geht hervor, dass in der EMV die größte durchschnittliche Potentialdifferenz von $\bar{V}_{S}^{+}$zu $\bar{V}_{S}^{-}$aufweist, wobei die Werte durch Abbilden der Fehlordnung fehlerbehaftet sind. Die Werte der InvariomVerfeinerung sind hingegen deutlich kleiner, da dort keine Kristalleffekte oder Wasserstoffbrückenbindung berücksichtigt sind.

\begin{tabular}{l|rrr} 
& \multicolumn{1}{|c}{ IV } & \multicolumn{1}{c}{ EMV } & \multicolumn{1}{c}{ GMV } \\
\hline $\bar{V}_{S}^{+}[\mathrm{e} / \AA ̊$ & 0,0283 & 0,0510 & 0,0440 \\
$\bar{V}_{S}^{-}[\mathrm{e} / \AA]$ & $-0,0540$ & $-0,0665$ & $-0,0612$ \\
$\Pi[\mathrm{e} / \AA]$ & 0,0424 & 0,0592 & 0,0522 \\
$\sigma_{\text {tot }}^{2}\left[\mathrm{e}^{2} / \AA^{2}\right]$ & 0,0058 & 0,0088 & 0,0089
\end{tabular}

Tabelle 7.6: Politzer-Analyse des elektrostatischen Potentials auf der Van-der-Waals-Oberfläche von Roxithromycin.

\subsection{Dipolmomente}

Durch Summation der Pseudoatomanteile können Dipolmomente in sehr kurzer Zeit berechnet werden. Als Referenz des Dipolmoments in der Gasphase wurde zusätzlich eine Single-Point-Berechnung mit der experimentellen Geometrie des GMV durchgeführt. Dabei wurde dieselbe Basis wie zur Erstellung von Invariomen verwendet [B3LYP/D95++(3df,3pd)].

In Tabelle 7.7 werden die Dipolmomente der drei Verfeinerungen der theoretischen Berechnung gegenüber gestellt. Dabei werden sowohl der Betrag $\mu_{\text {total }}$ als auch die richtungsabhängigen Komponenten $\mu_{X}, \mu_{Y}$ und $\mu_{Z}$ verglichen.

Während es eine sehr gute Übereinstimmung zwischen IV und der theoretischen Berechnung gibt, weichen die Ergebnisse von EMV und GMV um ca. 100\% vom theoretischen Wert ab. Eine aktuelle Untersuchung zu experimentellen Dipolmomenten [98] kam zu dem Schluss, dass des öfteren unrealistisch 


\begin{tabular}{l|c|rcr} 
Dipolmoment & SP & \multicolumn{1}{|c}{ IV } & EMV & GMV \\
\hline$\mu_{X}$ & 7,1 & 6,4 & $2,3(9)$ & $-3,2(8)$ \\
$\mu_{Y}$ & 1,7 & 1,5 & $13,1(9)$ & $12,9(10)$ \\
$\mu_{Z}$ & 6,1 & 7,6 & $-18,0(18)$ & $-15,2(18)$ \\
$\mu_{\text {total }}$ & 9,5 & 10,1 & $22,4(16)$ & $20,3(15)$
\end{tabular}

Tabelle 7.7: Dipolmomente einer Single-Point-Berechnung mit der Basis [B3LYP/D95++(3df,3pd)] (SP) und den Verfeinerungen. Das Dipolmoment ist in Debye [D] angegeben

große Erhöhungen der Dipolmomente aus experimentellen Ladungsdichtestudien hervorgehen. Deshalb ist Vorsicht bei der Interpretation der Ergebnisse geboten, da eine solche Abweichung durch Kristalleffekte zwar denkbar aber eher unwahrscheinlich ist. Zusätzliche theoretische Rechnungen könnten bei Einbeziehung von Kristalleffekten klären, ob es sich dabei um ein Artefakt oder eine realistische Berechnung im Rahmen der Standardabweichung handelt.

\subsection{Zusammenfassung}

Die subatomar aufgelöste Messung von Roxithromycin wurde am Deutschen Elektronen-Synchrotron durchgeführt. Im Anschluss an die sphärische Verfeinerung konnte durch eine „OMIT map“ der Wasserstoffatome gezeigt werden, dass alle Wasserstoffatompositionen experimentell beobachtet wurden. Der Invariom-Formalismus konnte erfolgreich angewendet werden und stellt eine entscheidende Modellverbesserung und eine gute Beschreibung der Elektronendichte dar. Durch die Berechnung von Differenz- und Deformationselektronendichtekarten konnte eine Rotationsfehlordnung entdeckt werden, welche gravierenden Einfluss auf die berechneten elektrostatischen Eigenschaften hat. Auf der ganzjährigen Titelseite der Zeitschrift Acta Crystallographica B des Jahres 2011 (hier Abbildung 7.11), welches sich auf die Untersuchung des Roxithromycin-Datensatzes [66] bezieht, wird das Phänomen wie folgt zusammengefasst:

„Das Titelbild zeigt ein Artefakt im elektrostatischen Potential, welches aus einer Multipolverfeinerung des Makrolid-Antibiotikums Roxithromycin abgeleitet wurde. Das Ignorieren der Fehlordnung in der Oxim-Kette (links) führt wegen der Flexibilität des Multipolmodells zu einem zu positiven Potential. Wird der Invariom-Formalismus, der einer theoretischen Elektronendichte- 


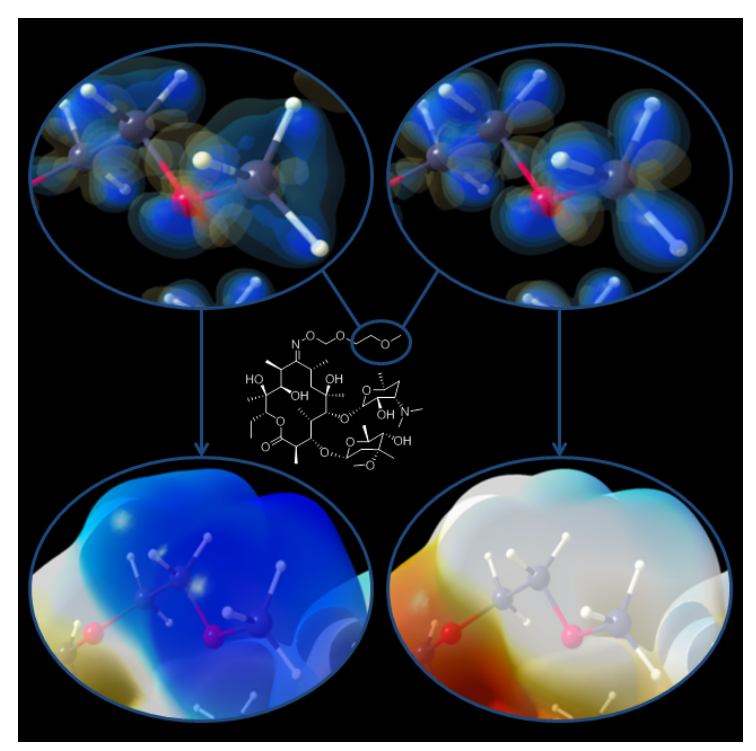

Abbildung 7.11: Ganzjähriges Titelbild der Ladungsdichtestudie von Roxithromycin in der Zeitschrift Acta Crystallographica B im Jahr 2011

verteilung entspricht, auf die fehlgeordnete Region angewendet (rechts), verschwindet das Artefakt. Das Verfahren soll die verlässliche Beschreibung der Elektronendichteverteilung trotz Fehlordnung erlauben.“

Das Verfeinerungsmodell wurde so angepasst, dass keine Multipolparameter der fehlgeordneten Gruppe verfeinert werden, sondern diese auf den theoretischen Werten der Invariom-Datenbank festgehalten werden. Durch Berechnung der dreidimensionalen Restelektronendichtekarten wurde zusätzlich die Notwendigkeit entdeckt, das Sauerstoffatom O27 der rotationsfehlgeordneten Methoxyruppe durch Gram-Charlier-Expansion anharmonisch zu verfeinern. Für die verschiedenen Modelle wurden elektrostatische Potentiale und Dipolmomente berechnet und die elektrostatischen Potentiale auf der molekularen Oberfläche nach der Politzer et al. [63] statistisch ausgewertet. 
Kapitel 8

\section{Fluorochinolon-Antibiotika}




\subsection{Pharmakologische Wirkung}

Fluorochinolone sind vollsynthetische Antibiotika und wirken als Inhibitoren des Enzyms Topoisomerase IV [99], welches in Bakterien, aber nicht in Eukaryoten vorkommt. Da die Resistenzentwicklung gegen die Fluorochinolone, von denen Ciprofloxacin der wohl bekannteste Vertreter ist, bereits zunimmt [100, 4], sind verstärkte Anstrengungen zur Aufklärung von Wirkung und Resistenzentwicklung notwendig. Strukturelle Arbeiten auf dem Gebiet wurden erst kürzlich veröffentlicht [101] und sind Teil aktueller Forschung. Dabei wurde erstmals die makromolekularen Bindungsstelle zwischen der Topoisomerase IV und verschiedenen Fluorochinolonen gefunden. Bei Topoisomerase IV handelt es sich um ein Enzym, welches in der Zelle für die Topologie der DNA verantwortlich ist. In seiner enzymatisch wirksamen Form liegt Topoisomerase IV als Dimer vor, wobei jedes Monomer in drei Domänen untergliedert werden kann:

1. Die N-terminale Domäne dient der Bindung von ATP.

2. Die zentrale Domäne wird auch als katalytische Domäne bezeichnet. Sie ist mit Hilfe eines Tyrosinrests für das Zerschneiden der DNA zuständig.

3. Die C-terminale Domäne variiert in der Länge und enthält ein nukleares Lokalisationssignal (NLS-Motiv). Sie ist teilweise phosphoryliert.

Topoisomerase IV ist in der Lage zusätzliche Windungen in einen DNA-Strang einzuführen, so dass positiv superspiralisierte DNA entspannt wird. In relaxierte DNA kann negative Verdrillung eingeführt werden. Diese sog. „negative Superspiralisierung“ der DNA erfolgt durch die Induktion von Doppelstrangbrüchen unter ATP-Verbrauch. Das Enzym ist außerdem in der Lage die superhelikale Struktur der DNA zu verändern und zwei verbundene DNA-Moleküle nach der Replikation zu dekatenieren, indem es einen Doppelstrang durch den anderen führt.

\subsection{Asphärische Atomformfaktoren}

Mit dem in Kapitel 5 beschriebenen Vorgehen wurden zur Erzeugung von Invariom-Datenbankeinträgen Modellverbindungen berechnet, welche nach den in Abschnitt 5.3 auf Seite 43 beschriebenen empirischen Regeln aus der chemischen Umgebung abgeleitet wurden. Die folgenden Pfeildiagramme zeigen die 
Strukturformeln der Moleküle, aus denen die asphärischen Atomformfaktoren der untersuchten Fluorochinolone berechnet wurden. $\mathrm{Zu}$ jeder Modellverbindung sind die zugehörigen INVARIOM-, SMILES- ${ }^{1}$ und IUPAC-Namen aufgeführt. Da sich die Fluorochinolone teilweise nur in den Seitengruppen unterscheiden, werden im folgenden die Invariom-Namen und Modellverbindungen für die Ringatome von Ciprofloxacin (Abbildung 8.3), Enoxacin (Abbildung8.4), Fleroxacin (Abbildung 8.5), Moxifloxacin (Abbildung 8.6), Nadifloxacin (Abbildung 8.7), Ofloxacin (Abbildung 8.8) und Sparfloxacin (Abbildung 8.9) gezeigt. Die Ringatome von Lomefloxacin und Norfloxacin werden dabei mit abgedeckt. Vollständige Tabellen der verwendeten asphärischen Streufaktoren und der Invariom-Namen und zugehörigen Modellverbindung aller Strukturen befinden sich im Anhang.

Das Ringsystem ist für alle Fluorochinolone außer Enoxacin identisch. Sie unterscheiden sich nur aufgrund ihrer Substituenten am Aromaten. Ausgehend von Ciprofloxacin, für dessen Ringsystem neun neue Modellverbindungen berechnet wurden, kann durch sechs zusätzliche Modellverbindungen auch Enoxacin beschrieben werden. Für Fleroxacin wurden zusätzlich vier, für Moxifloxacin drei, für Nadifloxacin fünf, für Ofloxacin eine und für Sparfloxacin zusätzlich drei Modellverbindungen benötigt. So ergeben sich insgesamt 31 verschiedene Modellverbindungen zur Beschreibung der Elektronendichte aller Ringatome der neun verschiedenen Fluorochinolone. Für jedes Zielmolekül wird der Zusammenhang zwischen dem Invariom-Namen und der zugehörigen Modellverbindung in einem Transfer-Diagramm gezeigt. In den Abbildungen 8.3 bis 8.9 sind die Modellverbindungen kreisförmig um das jeweils zentral positionierte Fluorochinolon verteilt. Die aus den Modellverbindungen transferierten Invariome sind mit einem roten Kreis markiert und alle explizit im Invariom-Namen enthaltenen Atome blau eingefärbt. Der Invariom-Name und die linearen Notationen der Moleküle nach SMILEs [75] sowie IUPAC ${ }^{2}$ sind zu jeder Modellverbindung angegeben.

\subsection{Strukturelle Eigenschaften}

Abbildung 8.1 zeigt die Strukturformeln der neun untersuchten Fluorochinolone. Auch wenn die Verbindungen im Kristall teilweise als Kationen oder

\footnotetext{
${ }^{1}$ Die Abkürzung SMILES steht im englischen für „Simplified Molecular Input Line Entry System“ und wurde von D. Weininger eingeführt [75].

${ }^{2}$ Es wird statt der deutschen die englische IUPAC-Nomenklatur verwendet, da die Modellverbindungen auch in der Datenbank nach englischer IUPAC-Nomenklatur benannt sind.
} 
Zwitterionen vorliegen, werden hier die ungeladenen Moleküle abgebildet. Bei den chiralen Verbindungen (Lomefloxacin, Nadifloxacin, Ofloxacin und Sparfloxacin) handelt es sich um racemische Gemische, weshalb je eine Bindung der Stereozentren geschwungen eingezeichnet ist. In Abbildung 8.2 wird das Pharmakophor gezeigt und die unterschiedlichen funktionellen Gruppen der untersuchten Fluorochinolone $\mathrm{R}_{1}, \mathrm{R}_{2}, \mathrm{R}_{3}$ und $\mathrm{X}$ verglichen. 
<smiles>O=C(O)c1cn(C2CC2)c2cc(N3CCNCC3)c(F)cc2c1=O</smiles><smiles>CCn1cc(C(=O)O)c(=O)c2cc(F)c(N3CCN[C@H](C)C3)c(F)c21</smiles><smiles>CCn1cc(C(=O)O)c(=O)c2cc(F)c(N3CCNCC3)cc21</smiles><smiles>CCOCCN1C(C(=O)O)=CN(CC)c2nc(N3CCNCC3)c(F)cc21</smiles><smiles>COc1c(N2CC3CCCNC3C2)c(F)cc2c(=O)c(C(=O)O)cn(C3CC3)c12</smiles><smiles>CC1=CN2c3c(cc(F)c(N4CCN(C)CC4)c3OC1)C(=O)C2C(=O)O</smiles><smiles>CN1CCN(c2c(F)cc3c(=O)c(C(=O)O)cn(CCF)c3c2F)CC1</smiles><smiles>C[C@@H]1CCc2c(N3CCC(O)CC3)c(F)cc3c(=O)c(C(=O)O)cn1c23</smiles><smiles>CC1=N[C@H](C)CN(c2c(F)c(N)c3c(=O)c(C(=O)O)cn(C4CC4)c3c2F)C1</smiles>

Abbildung 8.1: Strukturformeln der neun untersuchten Fluorochinolone. Die Stereozentren chiraler Verbindungen wurden markiert und je eine Bindung der Stereozentren geschwungen eingezeichnet, da die chiralen Verbindungen als Racemat vorliegen.

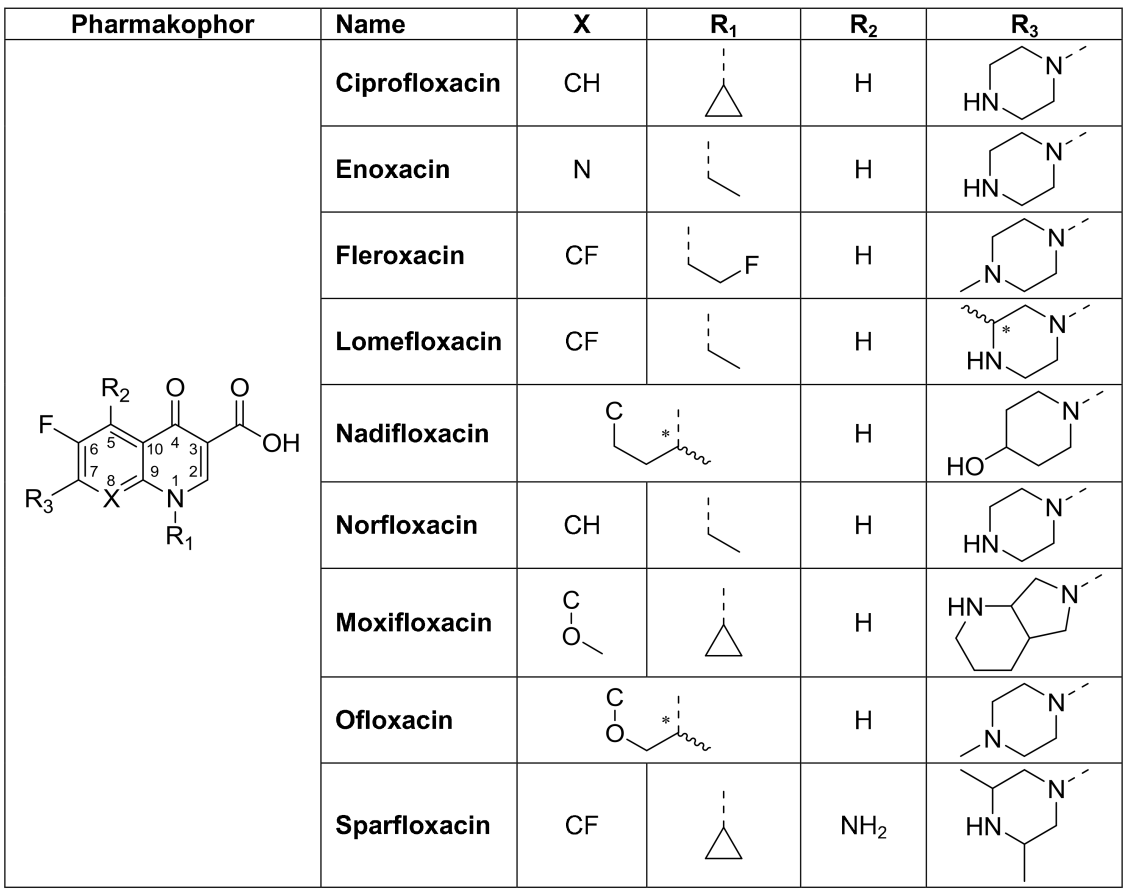

Abbildung 8.2: Überblick der verschiedenen Substitutionsmuster am Pharmakophor der neun untersuchten Fluorochinolone. Die Atome des aromatischen Rings sind der IUPAC-Konvention entsprechend nummeriert. 


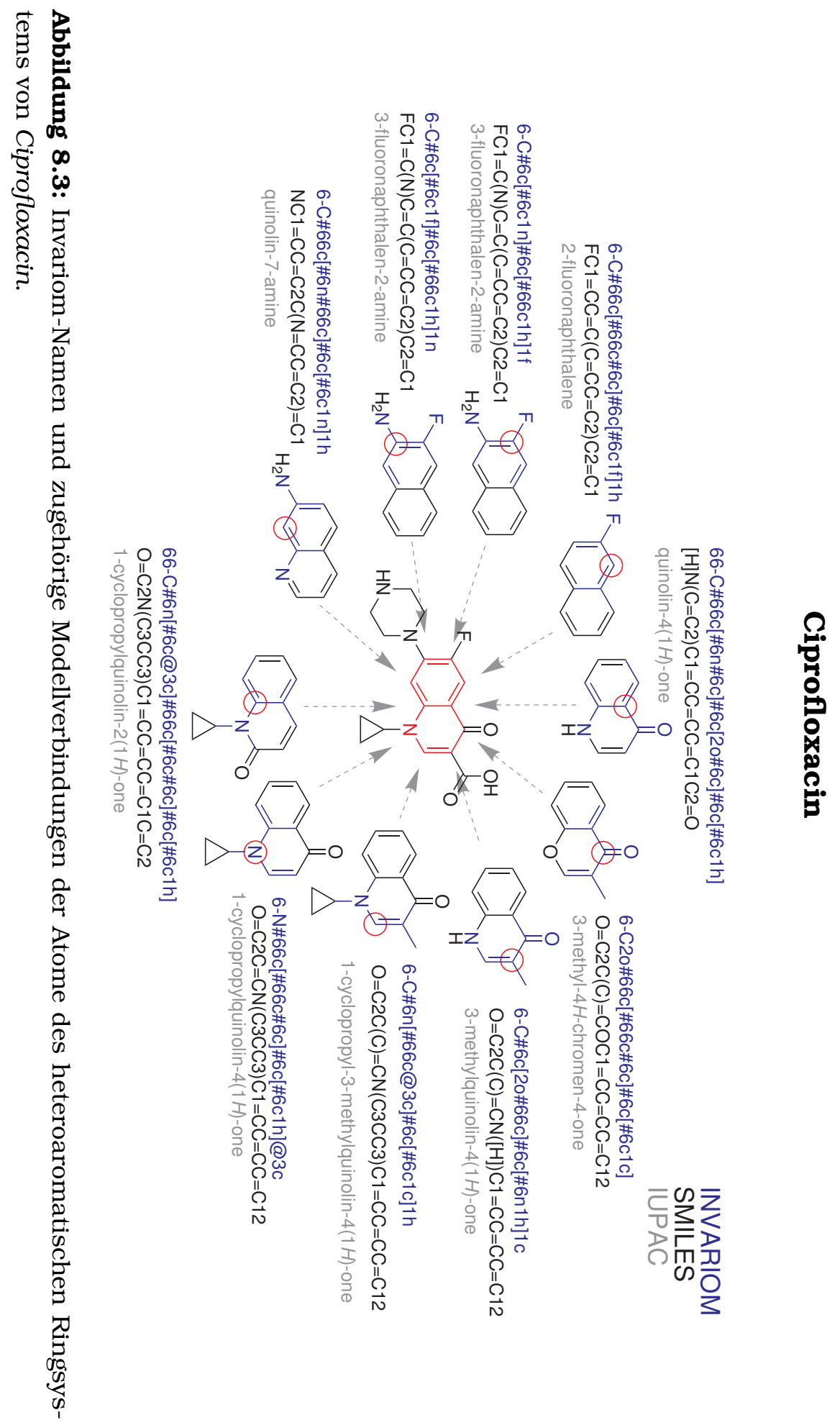




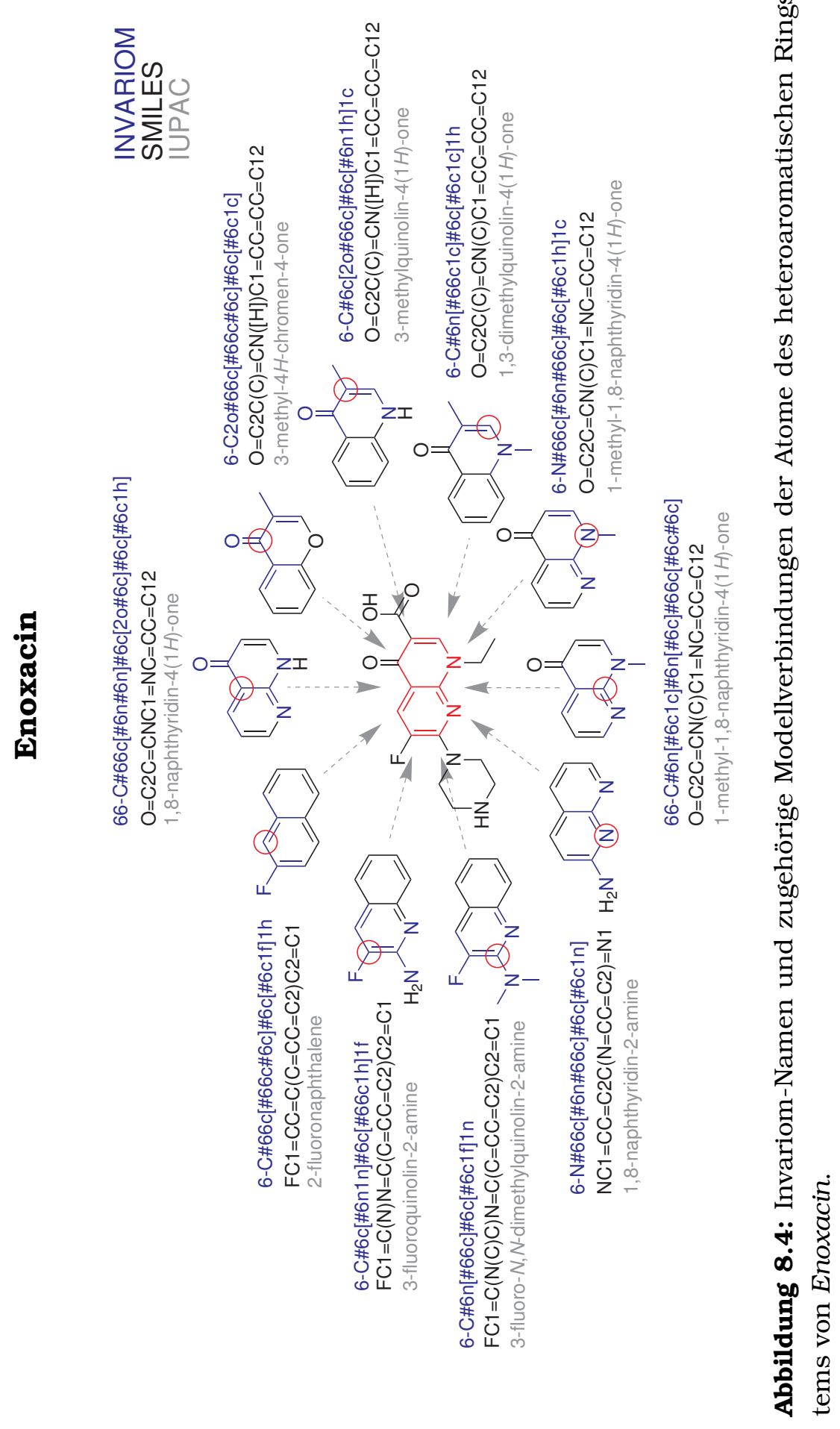




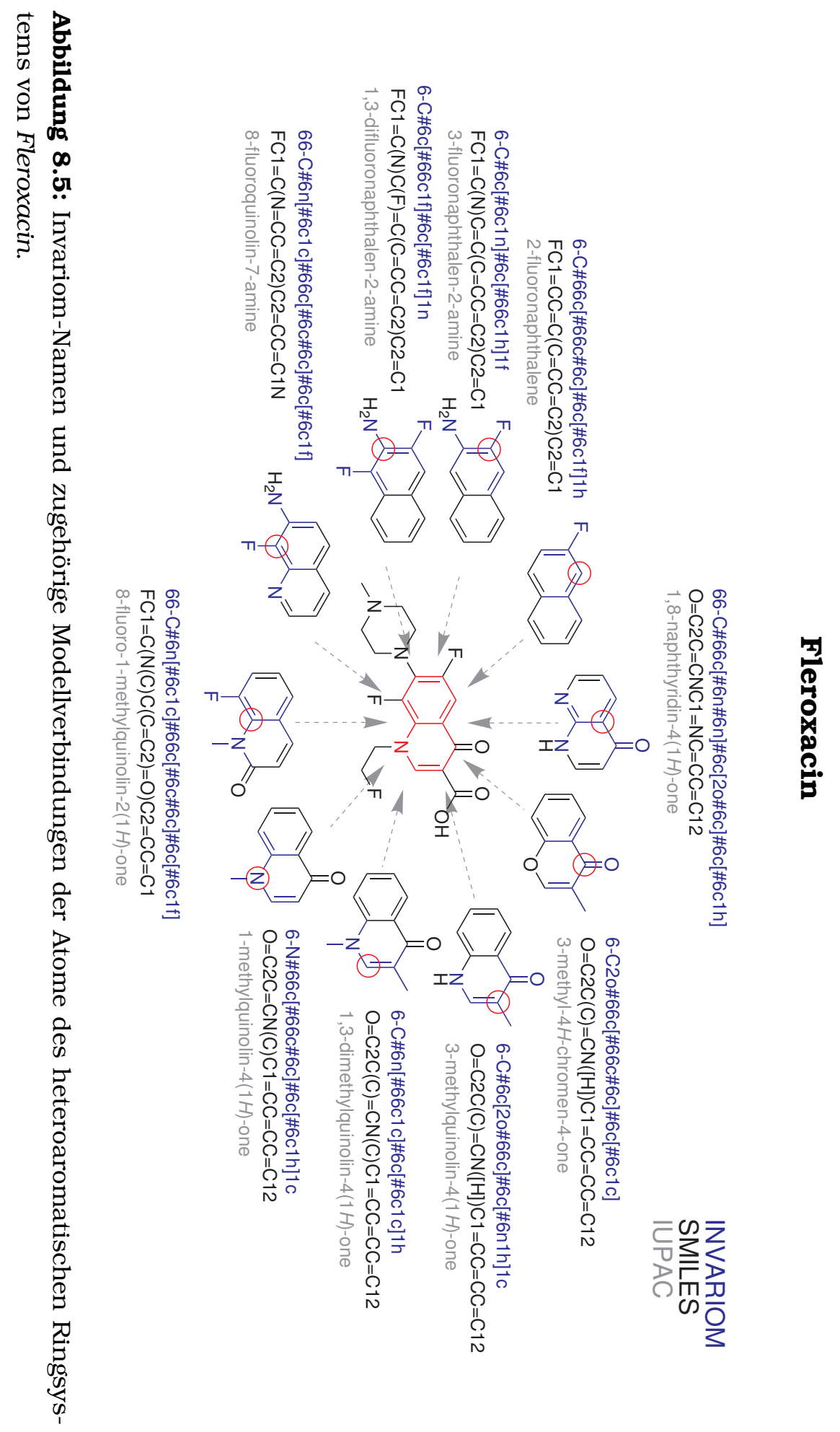




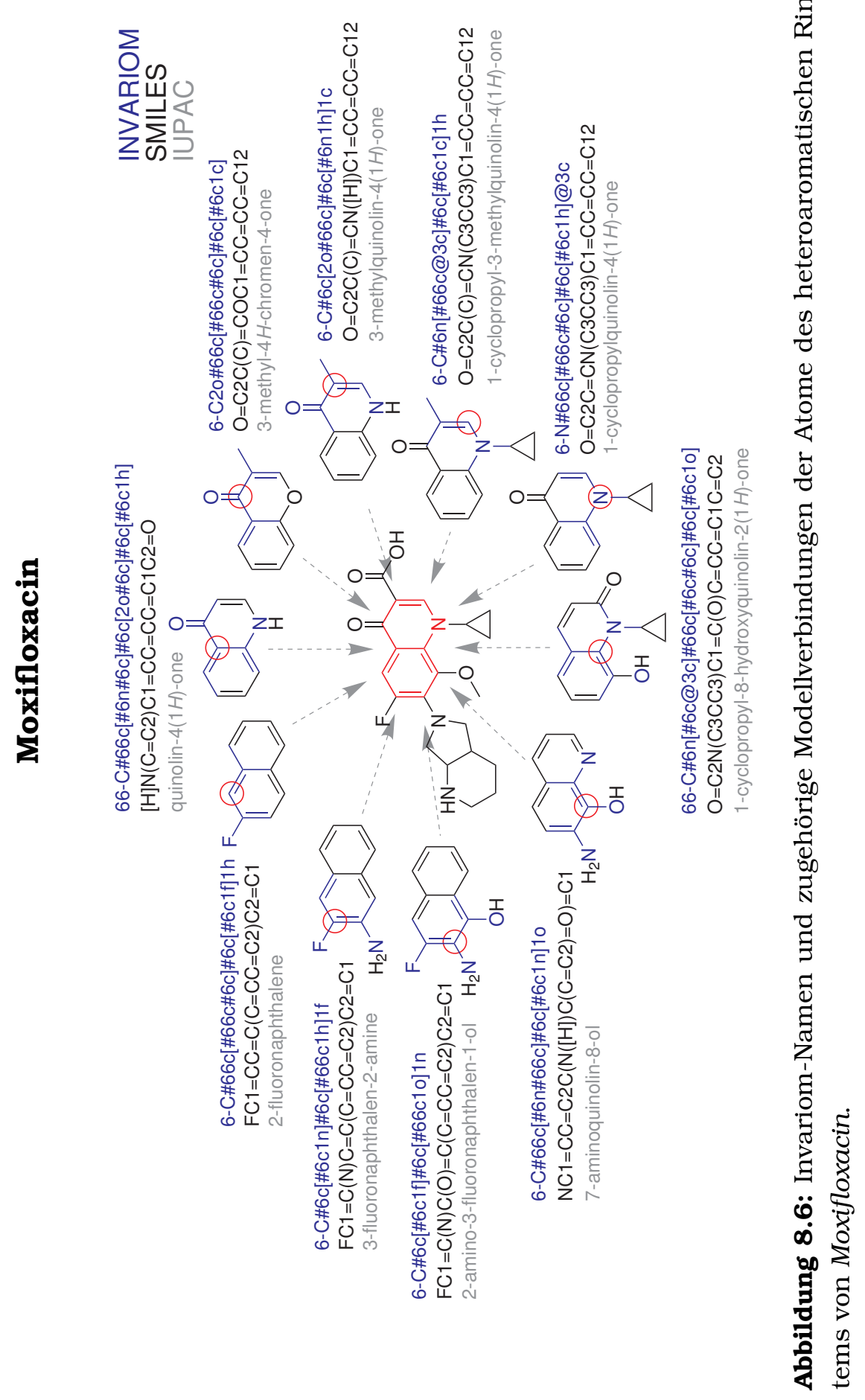




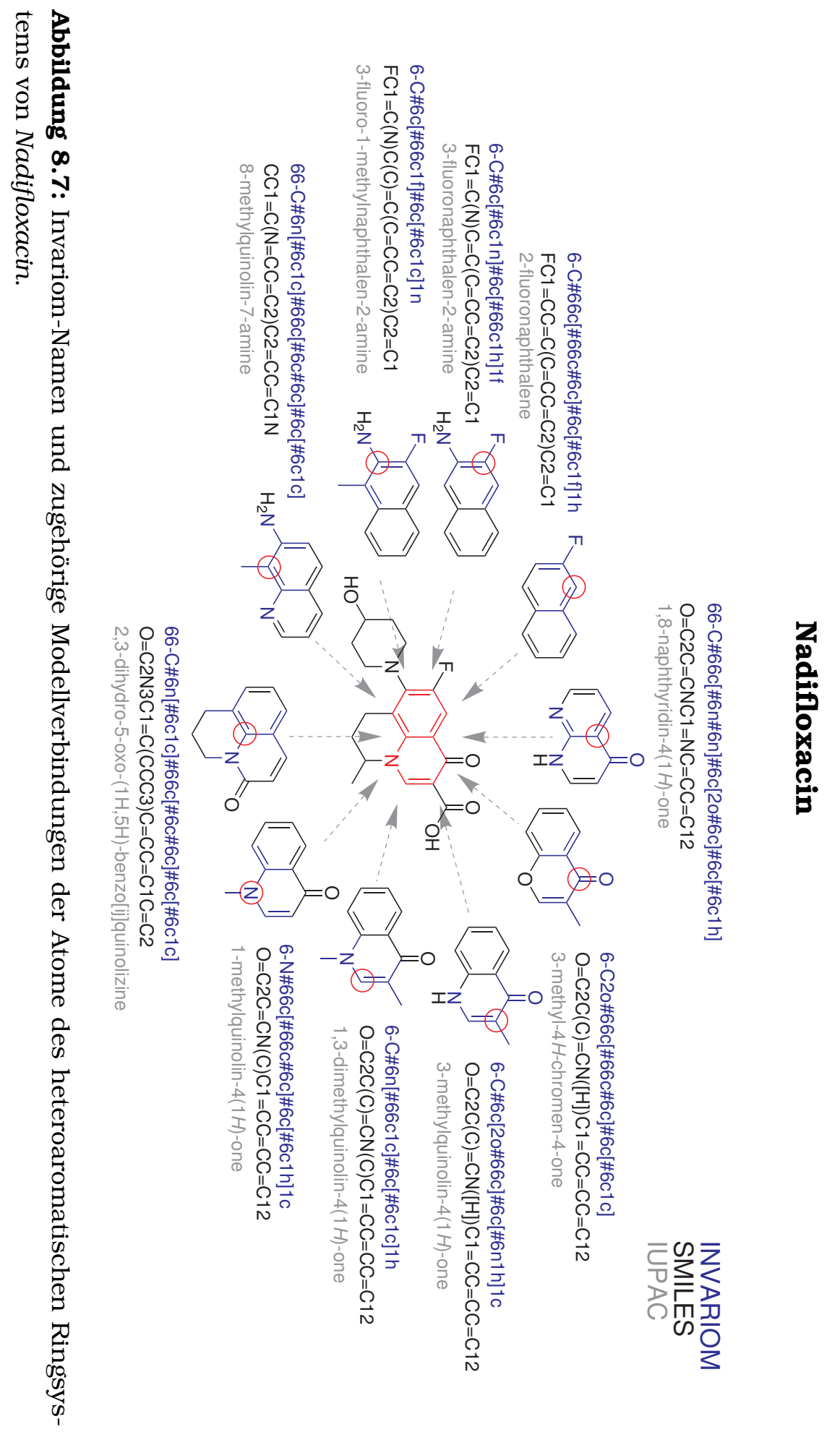




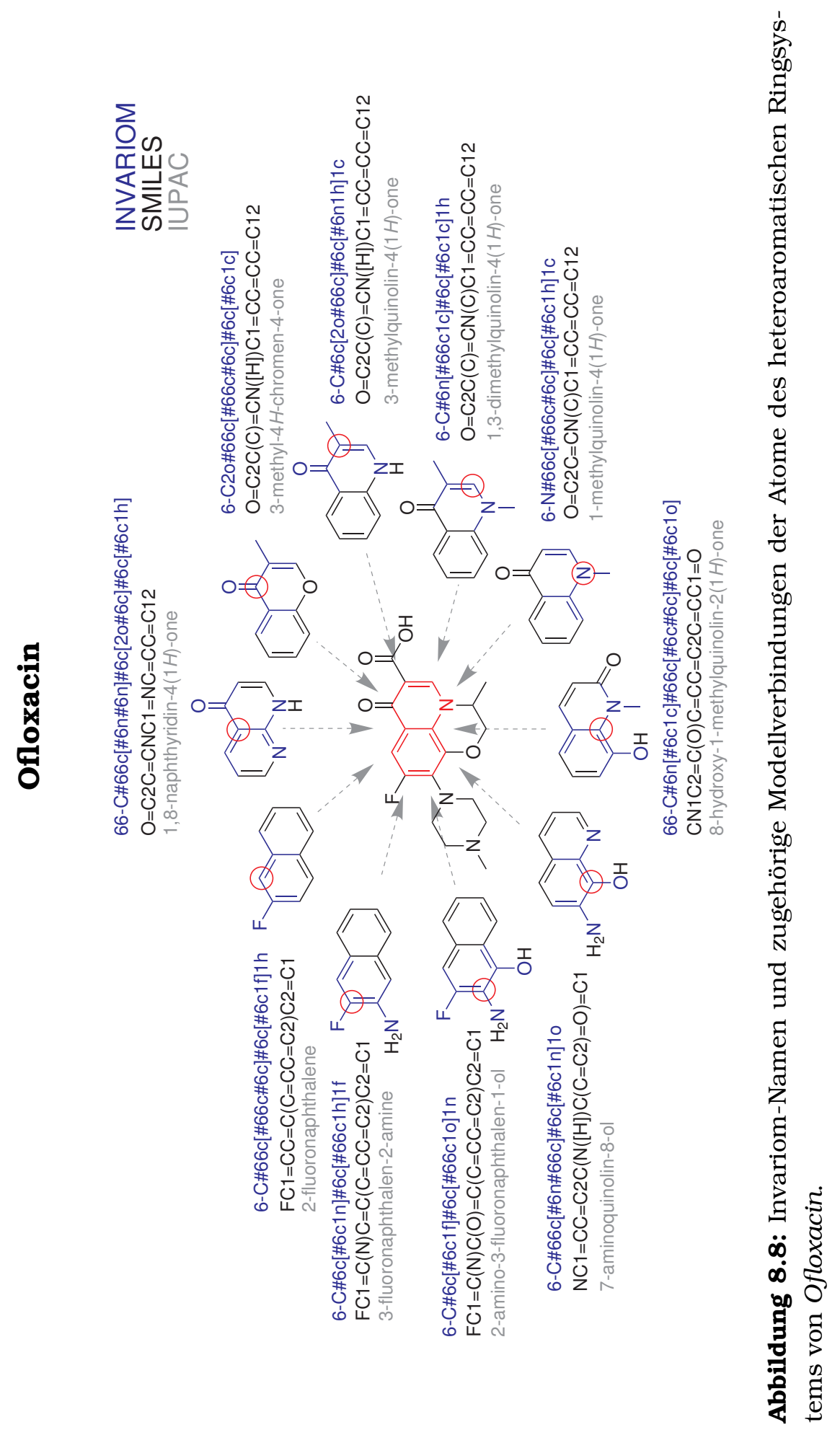




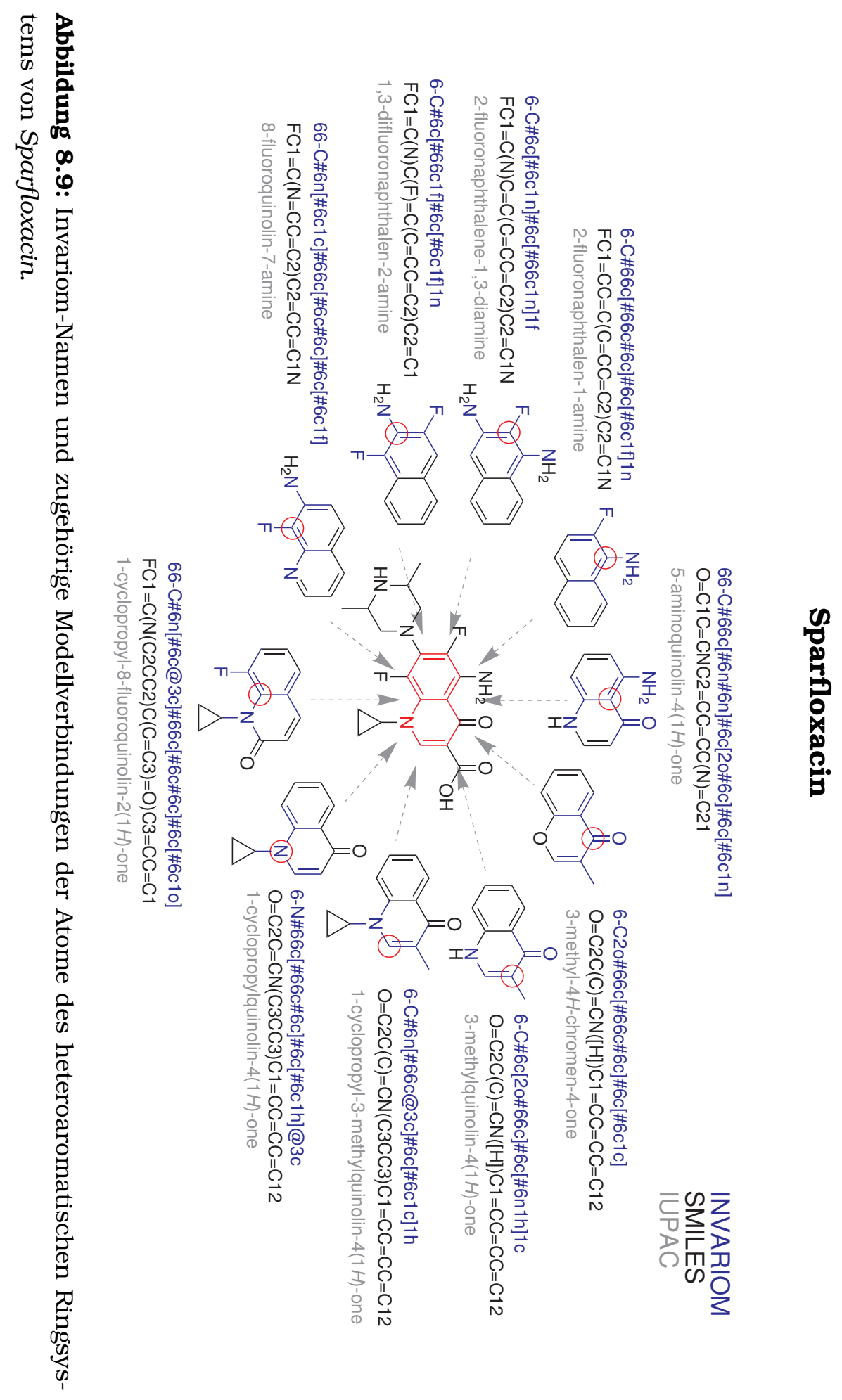




\subsection{Verwendete Datensätze}

Es wurden zwölf Kristallstrukturen von neun verschieden substituierten Chinolin-Derivate untersucht. Bei Ciprofloxacin, Enoxacin und Norfloxacin wurden jeweils zwei Strukturen untersucht, die sich in der Solvatisierung oder der Protonierung unterscheiden. Die in den Zeitschriften Acta Crystallographica $C$ oder $E$ verfügbaren Strukturfaktoren von Enoxacin $3 \mathrm{H}_{2} \mathrm{O}$ [102], Fleroxacin $\cdot \mathrm{H}_{2} \mathrm{O}$ · $\mathrm{HCl}$ [103], Moxifloxacin.0, $5 \mathrm{H}_{2} \mathrm{O} \cdot 0,5 \mathrm{CH}_{3} \mathrm{OH} \cdot \mathrm{HCl}$ [104] und Sparfloxacin. $3 \mathrm{H}_{2} \mathrm{O}$ [105] wurden von der Website der Journale bezogen. Ausführliche Details der Messungen dieser Strukturen sind in den oben genannten Literaturstellen zu finden.

Die bereits bekannten Strukturen von Ciprofloxacin. $6 \mathrm{H}_{2} \mathrm{O}$ [106], Ciprofloxacin 1,4 $\cdot \mathrm{H}_{2} \mathrm{O} \cdot \mathrm{HCl}$ [107], Enoxacin $1,7 \mathrm{H}_{2} \mathrm{O}$ [108], Nadifloxacin.0,5 $\mathrm{H}_{2} \mathrm{O}$ [109], Norfloxacin-Anhydrat [110], Norfloxacin $2 \mathrm{H}_{2} \mathrm{O}$ [111] und Ofloxacin-Anhydrat [112] wurden erneut gemessen. Bei jeder Messung wurde versucht subatomare Auflösung ( $\sin \theta / \lambda \geq 1,0 \AA^{-1}$ bzw. $\mathrm{d} \leq 0,5 \AA$ ) zu erreichen, was bei Ciprofloxacin 6 $\mathrm{H}_{2} \mathrm{O}$ und Enoxacin $1,7 \mathrm{H}_{2} \mathrm{O}$ gelang. Bedingt durch Kristallgrösse und -qualität konnte in den anderen Fällen keine so hohe Auflösung erreicht werden, aber zumindest die Auflösung der in der Literatur veröffentlichten Messungen übertroffen werden. Das gemessene Solvat von Lomefloxacin $2 \mathrm{H}_{2} \mathrm{O} \cdot 0,95 \mathrm{Cl}^{-}$war bisher unbekannt. Von den gemessenen Strukturen sind die kristallographischen Daten in den Tabellen 8.1 und 8.2 aufgeführt.

\subsection{Vorgehen zur Struktur-Modellierung}

Alle neu gemessenen Strukturen wurden mit SHELXS gelöst. Für das Pharmakophor wurde in allen Verbindungen eine einheitliche Zählweise verwendet, die auf dem IUPAC-Nummerierungsschema der Chinolin-Struktur basiert. Die Strukturen wurde mit dem Modell unabhägiger Atome (IAM) in SHELXL [43] verfeinert. Außer im Fall von Lomefloxacin $2 \mathrm{H}_{2} \mathrm{O} \cdot 0,95 \mathrm{Cl}^{-}$und Nadifloxacin.0,5 $\mathrm{H}_{2} \mathrm{O}$ wurden die Auslenkungsparameter und Positionen aller Atome in SHELXL frei verfeinert.

Um die Ergebnisse der SHELXL-Verfeinerungen in XD [90] zu reproduzieren, wurden die Werte der anomalen Dispersion $\left[\Delta f^{\prime}(\lambda)\right.$ und $\left.\Delta f^{\prime \prime}(\lambda)\right]$ aller Atome für die entsprechende Wellenlänge angepasst. Für Chlorid-Ionen wurde der Atomformfaktor $\left(\mathrm{Cl}^{-}\right)$nach Clementi und Roetti [29] verwendet. Als Kriterium für beobachtete Reflexe wurde $[I>3 \sigma(I)]$ verwendet, was dem Standard-Wert von 
XD entspricht. Anschließend wurden mit InVARIOMTOOL [49] die asphärischen Atomformfaktoren aus der Invariom-Datenbank transferiert. Für die asphärischen Atomformfaktoren wurden die in Abschnitt 8.2 vorgestellt Modellverbindungen verwendet. Die asphärischen Atomformfaktoren konnten mit dem Programm INVARIOMTOOL [49] automatisiert für alle neun Fluorochinolone aus der Invariom-Datenbank transferiert werden. Im Fall der fehlgeordneten Struktur von Lomefloxacin $2 \mathrm{H}_{2} \mathrm{O} \cdot 0,95 \mathrm{Cl}$ wurden die Invariom-Namen mit MOLECOOLQT [72] erstellt, der Transfer erfolgte anschließend mit InvaRIOMTool [49]. Da die Anzahl der Parameter in der Verfeinerung dabei nicht erhöht wird, konnten sie auch für die atomar aufgelösten Datensätze durchgeführt werden.

Für die Datensätze von Ciprofloxacin· $6 \mathrm{H}_{2} \mathrm{O}$, Enoxacin· $1,7 \mathrm{H}_{2} \mathrm{O}$, Lomefloxacin·2 $\mathrm{H}_{2} \mathrm{O} \cdot 0,95 \mathrm{Cl}$, Nadifloxacin $0,5 \mathrm{H}_{2} \mathrm{O}$ und Ofloxacin-Anhydrat ergab die Gewichtung von $1 / \sigma^{2}\left[F_{o}^{2}(\vec{h})\right]$ den besten gewichteten R-Wert $\left[R_{w}(F)\right]$ bei der InvariomVerfeinerung in XD. Für die anderen Strukturen wurde das Zwei-ParameterGewichtungsschema verwendet und die in SHELXL [43] berechneten Gewichtungparameter $\mathbf{a}$ und $\mathbf{b}$ für die Invariom-Verfeinerung mit DRKPLOT, welches im Programm WINGX [113] implementiert ist, nachoptimiert.

Die genauere Beschreibung der molekularen Dichte erhöht dabei in allen zwölf Fällen die Übereinstimmung von Beobachtung und Modell und ermöglicht die Rekonstruktion der molekulare Elektronendichte, um die chemischen Eigenschaften zu berechnen.

\subsection{Datenreduktion und Parametrisierung der Invariom- Verfeinerung}

\subsubsection{Ciprofloxacin $6 \mathrm{H}_{2} \mathrm{O}$}

Farblose Kristalle wurden durch langsame Verdunstung einer gesättigten wässrigen Lösung kristallisiert. Die Messung von Ciprofloxacin $6 \mathrm{H}_{2} \mathrm{O}$ erfolgte mit der MoK $\alpha$-Microsource. Der für die Messung verwendete Kristall hatte die Dimension von $0,15 \times 0,08 \times 0,07 \mathrm{~mm}$. Bei einer Temperatur von $100 \mathrm{~K}$ wurde in fünf Tagen eine Auflösung von $\sin \theta / \lambda=1,16 \AA^{-1}$ erreicht. Der Detektorabstand betrug 41,11 mm und die Abdeckung des reziproken Raums 97,9\%. Dabei wurde eine Redundanz von 5,24 und ein interner R-Wert von $R_{\text {int }}=2,5 \%$ erreicht. Die Integration der Daten erfolgte mit SAINT (Version 7.60A) [114], wobei bereits eine Korrektur für Reflexe mit schrägem Einfallswinkel auf den Detek- 
tor (engl. oblique incidence) durchgeführt wurde. Zur Skalierung und empirischen Absorptionskorrektur wurde das Programm SADABS [115] verwendet.

Ausgehend von den Ergebnissen der Invariom-Verfeinerung wurden die anisotropen Auslenkungsparameter für Wasserstoffatome in das Strukturmodell implementiert. Dafür wurde der SHADE2-Server [91, 92] verwendet und das Ciprofloxacin-Molekül der asymmetrischen Einheit in zwei Fragmente unterteilt, für die voneinander unabhägig eine TLS-Analyse (engl. Translation, Libration, Screw) starrer Körper zur Abschätzung der anisotropen Auslenkungsparamenter von Wasserstoffatomen durchgeführt wurde. Als erstes Fragment wurde das Pharmakophor inklusive der Reste $R_{1}, R_{2}$ und $X$ verwendet. Der Piperazin-Ring an Position $\mathrm{R}_{3}$ wurde als zweiter starrer Körper definiert (siehe Abbildung 8.2).

Die Ladungsdichtestudie des Datensatzes wurde bereits zusammen mit der Untersuchung des Ciprofloxacin $1,4 \mathrm{H}_{2} \mathrm{O} \cdot \mathrm{HCl}$ Datensatzes veröffentlicht [116].

\subsubsection{Ciprofloxacin $1,4 \mathrm{H}_{2} \mathrm{O} \cdot \mathrm{HCl}$}

Kommerziell bezogene Tabletten von Ciprofloxacin-Hydrochlorid (Ciprobay ${ }^{\circledR}$ ) wurden in Wasser gelöst. Durch langsames Verdunsten der Lösung bildeten sich nadelförmige Kristalle. Ein Kristall der Größe von $0,62 \times 0,31 \times 0,01 \mathrm{~mm}$ wurden am Protein-Messplatz des Australian Synchrotron gemessen. Bei 100 $\mathrm{K}$ und einer Wellenlänge von $\lambda=0,7662 \AA$ wurden je drei Durchläufe bei $2 \theta=$ $0^{\circ}$ und $2 \theta=20^{\circ}$ gemessen, wovon die Durchläufe eins, drei, fünf und sechs verwendet wurden. Der Datensatz wurden mit XDS [87] integriert. Nach einer Dateikonvertierung des XDS_ASCII.HKL mit dem Programm XDS2SAD ${ }^{3}$ in die Datei xds.sad wurde die Skalierung und eine empirische Absorptionskorrektur mit SADABS [115] durchgeführt. Durch die Verwendung von SynchrotronStrahlung konnte eine Auflösung von $(\sin \theta / \lambda)_{\max }=0,84 \AA^{-1}$ bzw. $\mathrm{d}=0,61 \AA$ erreicht werden.

Nach dem Invariom-Transfer wurden $\mathrm{X}-\mathrm{H}$-Abstände vor der Invariom-Verfeinerung auf die theoretischen Werte aus der Invariom-Datenbank gesetzt und die Positionen von Wasserstoffatomen anschließend in Abhängigkeit der Position der gebundenen Atome verfeinert. Auslenkungsparameter aller NichtWasserstoffatome wurden anisotrop und die der Wasserstoffatome isotrop verfeinert.

\footnotetext{
${ }^{3}$ XDs2SAD wurde von Prof. G. M. Sheldrick geschrieben.
} 


\subsubsection{Enoxacin $1,7 \mathrm{H}_{2} \mathrm{O}$}

Die Daten von Enoxacin wurden mit der MoK $\alpha$-Microsource bis zu einer Auflösung von $(\sin \theta / \lambda)_{\max }=1,02 \AA^{-1}$ gemessen. Dabei wurde eine Vollständigkeit von $99,8 \%$ bei einem internen $R$-Wert von $4,2 \%$ erreicht.

Das von Yoon et al. [108] veröffentlichte Strukturmodell ${ }^{4}$ eines Enoxacin-Methanol-Solvats stellte sich bei Verwendung der gleichen Kristallisationsbedingungen und identischer Zelle als falsch heraus. Neben den zwei EnoxacinMolekülen befinden sich nur Wassermoleküle in der asymmetrischen Einheit, von denen zwei voll, zwei halb und eines zu $40 \%$ besetzt sind.

Ausgehend von den Ergebnissen der Invariom-Verfeinerung wurden anisotrope Auslenkungsparameter für Wasserstoffatome in das Strukturmodell implementiert. Dafür wurde der SHADE2-Server [91, 92] verwendet und jedes Enoxacin Molekül der asymmetrischen Einheit in zwei Fragmente unterteilt, für die voneinander unabhängig eine TLS-Analyse (engl. Translation, Libration, Screw) starrer Körper zur Abschätzung der anisotropen Auslenkungsparameter von Wasserstoffatomen durchgeführt wurde. Als erstes Fragment wurde das Pharmakophor inklusive der Reste $R_{1}, R_{2}$ und $X$ (siehe Abbildung 8.2) verwendet. Der Piperazin-Ring an Position $R_{3}$ wurde als zweiter starrer Körper definiert.

\subsubsection{Enoxacin $3 \mathrm{H}_{2} \mathrm{O}$}

Die Verfeinerung von Enoxacin $3 \mathrm{H}_{2} \mathrm{O}$ erfolgte in SHELXL ausgehend vom publizierten Strukturmodell [102].

Vor der Invariom-Verfeinerung wurden X-H-Abstände auf die Werte der Invariom-Datenbank gesetzt und die Positionen der Wasserstoffatome anschließend in Abhängigkeit der jeweils gebundenen Atome verfeinert. Die Auslenkungsparameter aller Nicht-Wasserstoffatome wurden anisotrop und die der Wasserstoffatome isotrop verfeinert.

\subsubsection{Fleroxacin $\cdot \mathrm{H}_{2} \mathrm{O} \cdot \mathrm{HCl}$}

Die Verfeinerung von Fleroxacin $\mathrm{H}_{2} \mathrm{O} \cdot \mathrm{HCl}$ erfolgte in sHELXL ausgehend vom publizierten Strukturmodell [103].

\footnotetext{
${ }^{4}$ CSD-code: ITEKUN
} 
Vor der Invariom-Verfeinerung wurden $\mathrm{X}-\mathrm{H}$-Abstände auf die Werte der Invariom-Datenbank gesetzt und die Positionen der Wasserstoffatome anschließend in Abhängigkeit der jeweils gebundenen Atome verfeinert. Die Auslenkungsparameter aller Nicht-Wasserstoffatome wurden anisotrop und die der Wasserstoffatome isotrop verfeinert.

\subsubsection{Lomefloxacin $2 \mathrm{H}_{2} \mathrm{O} \cdot 0,95 \mathrm{Cl}^{-}$}

Am Protein-Messplatz X10SA des SLS konnte ein sehr kleiner nadelförmiger Kristall von Lomefloxacin $2 \mathrm{H}_{2} \mathrm{O} \cdot 0,95 \mathrm{Cl}^{-}$der Dimension $0,05 \times 0,01 \times 0,01 \mathrm{~mm}$ gemessen werden. Dafür wurde eine Wellenlänge von $\lambda=0,8000 \AA$ gewählt und eine maximale Auflösung von $(\sin \theta / \lambda)_{\max }=0,54 \AA^{-1}$ erreicht. Es handelt sich um ein bisher unbekanntes Solvat von Lomefloxacin. Die Struktur wurde mit direkten Methoden mit dem Programm sHelXs gelöst [43] und kristallierte in der Raumgruppe P2 1 /c. Wegen Fehlordnung des Piperazin-Rings durch Überlagerung beider Enantiomere wurde versuchsweise in der Raumgruppen P2 1 gelöst und verfeinert. Allerdings blieb die Fehlordnung auch in der Raumgruppe geringerer Symmetrie bestehen und die Verfeinerung anisotroper Auslenkungsparameter war instabil, weshalb nur $\mathrm{P} 2_{1} / \mathrm{c}$ die richtige Raumgruppe sein kann. Die Fehlordnung der beiden Enantiomere wurde mit einer freien Variablen auf 0.626(14) zu 0.384(14) verfeinert. Da sich die Population der Fehlordnung auf die asymmetrischen Einheit bezieht, soll nicht darüber hinwegtäuschen, dass die Enantiomere im Verhältnis 1:1 vorliegen, wie aus der zentrosymmetrischen Raumgruppe zu ersehen ist. Darüber wurden für die anisotropen Auslenkungsparameter der fehlgeordneten Atome „restraints“eingeführt. In der Invariom-Verfeinerung wurden nur die Positionen und Auslenkungsparameter der Atome des nicht fehlgeordneten Teils verfeinert. Für den fehlgeordneten Teil wurden die Auslenkungsparameter aus SHELXL beibehalten. $\mathrm{X}-\mathrm{H}$-Abstände wurden vor der Verfeinerung auf die theoretischen Werte aus der Invariom-Datenbank gesetzt und festgehalten.

\subsubsection{Moxifloxacin $\cdot 0,5 \mathrm{H}_{2} \mathrm{O} \cdot 0,5 \mathrm{CH}_{3} \mathrm{OH} \cdot \mathrm{HCl}$}

Die Verfeinerung von Moxifloxacin-0,5 $\mathrm{H}_{2} \mathrm{O} \cdot 0,5 \mathrm{CH}_{3} \mathrm{OH} \cdot \mathrm{HCl}$ erfolgte in SHELXL ausgehend vom publizierten Strukturmodell [104]. In der Invariom-Verfeinerung wurden die X-H-Abstände auf die theoretischen Werte aus der Invariom-Datenbank gesetzt und festgehalten. Auslenkungsparameter von Wasserstoffatomen wurden isotrop und von Nicht-Wasserstoffatomen anisotrop verfeinert. 


\subsubsection{Nadifloxacin $\cdot 0,5 \mathrm{H}_{2} \mathrm{O}$}

Kristalle mit den Dimensionen 0,12×0,07×0,05 mm von Nadifloxacin.0,5 $\mathrm{H}_{2} \mathrm{O}$ wurden mit $\mathrm{CuK} \alpha$-Strahlung der Drehanode gemessen. Bis zu einer Auflösung von $(\sin \theta / \lambda)_{\max }=0,62 \AA^{-1}$ bei einer Vollständigkeit von $98,6 \%$ und einer Redundanz von 8,99 wurde ein interner R-Wert von 3,3\% erreicht. In der SHELXL-Verfeinerung wurden die mit Positionen der Wasserstoffatome im Reitermodell in Abhängigkeit der jeweils gebundenen Atome verfeinert. Die Auslenkungsparameter aller Wasserstoffatome wurden dabei mit der Bedingung $U_{\text {eq }}(\mathrm{X})=1,5 \cdot U_{\text {iso }}(\mathrm{H})$ bzw. $U_{\text {eq }}(\mathrm{X})=1,2 \cdot U_{\text {iso }}(\mathrm{H})$ in Abhängigkeit vom jeweils gebundenen Atom $(\mathrm{X})$ verfeinert.

In der Invariom-Verfeinerung wurden keine Auslenkungsparameter von Wasserstoffatomen verfeinert. Nicht-Wasserstoffatome wurden anisotrop verfeinert. $\mathrm{X}-\mathrm{H}$-Abstände wurden in der Verfeinerung auf die theoretischen Werte aus der Invariom-Datenbank und die Positionen von Wasserstoffatomen in Abhängigkeit der Position des jeweils gebundenen Atoms verfeinert.

\subsubsection{Norfloxacin-Anhydrat}

Der Datensatz von Norfloxacin-Anhydrat wurde aufgrund des kleinen Kristalls mit Dimensionen von $0,10 \times 0,04 \times 0,04 \mathrm{~mm}$ ebenfalls mit $\mathrm{CuK} \alpha$-Strahlung der Drehanode gemessen. Hier konnte bis zu einer Auflösung von $(\sin \theta / \lambda)_{\max }=$ $0,60 \AA^{-1}$ und eine Vollständigkeit von $96,3 \%$ erreicht werden. Der interne RWert betrug 7,5 \% für eine Redundanz von 6,21.

Vor der Invariom-Verfeinerung wurden $\mathrm{X}-\mathrm{H}$-Abstände auf die Werte der Invariom-Datenbank gesetzt und die Positionen der Wasserstoffatome anschließend in Abhängigkeit der jeweils gebundenen Atome verfeinert. Die Auslenkungsparameter aller Nicht-Wasserstoffatome wurden anisotrop und die der Wasserstoffatome isotrop verfeinert.

\subsubsection{Norfloxacin.2 $\mathrm{H}_{2} \mathrm{O}$}

Bei Kristalldimensionen von $0,20 \times 0,05 \times 0,05 \mathrm{~mm}$ konnte mit der MoK $\alpha$-Microsource eine Auflösung von $(\sin \theta / \lambda)_{\max }=0,77 \AA^{-1}$ mit einer Vollständigkeit von 98,6\% und einer Redundanz von 5,32 erreicht werden. Der interne R-Wert betrug $3,7 \%$.

Vor der Invariom-Verfeinerung wurden $\mathrm{X}-\mathrm{H}$-Abstände auf die Werte der Invari- 
om-Datenbank gesetzt und die Positionen der Wasserstoffatome anschließend in Abhängigkeit der jeweils gebundenen Atome verfeinert. Die Auslenkungsparameter aller Nicht-Wasserstoffatome wurden anisotrop und die der Wasserstoffatome isotrop verfeinert.

\subsubsection{Ofloxacin-Anhydrat}

Ein Kristall von Ofloxacin wurde an der AgK $\alpha$-Microsource bis zu einer Auflösung von $(\sin \theta / \lambda)_{\max }=0,78 \AA^{-1}$ gemessen. Die Kristalldimensionen betrugen $0,20 \times 0,19 \times 0,17 \mathrm{~mm}$. Bei einer Vollständigkeit von 99,8\% und einer Redundanz von 12,27 betrug der interne R-Wert 5,4\%.

\subsubsection{Sparfloxacin $3 \mathrm{H}_{2} \mathrm{O}$}

Die Verfeinerung von Sparfloxacin $3 \mathrm{H}_{2} \mathrm{O}$ erfolgte in SHELXL ausgehend vom publizierten Strukturmodell [105].

Vor der Invariom-Verfeinerung wurden $\mathrm{X}-\mathrm{H}$-Abstände auf die Werte der Invariom-Datenbank gesetzt und die Positionen der Wasserstoffatome anschließend in Abhängigkeit der jeweils gebundenen Atome verfeinert. Die Auslenkungsparameter aller Nicht-Wasserstoffatome wurden anisotrop und die der Wasserstoffatome isotrop verfeinert. 


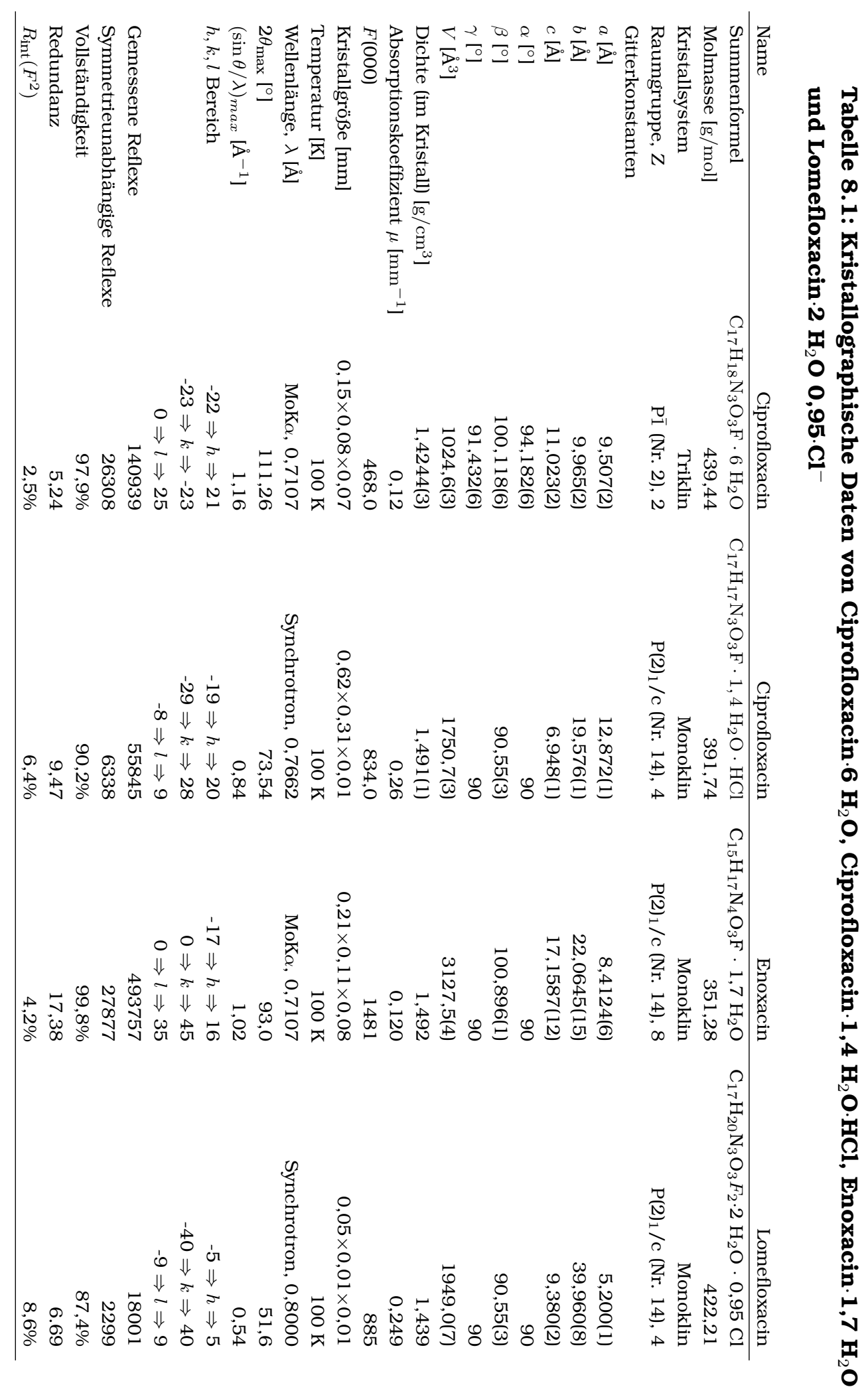




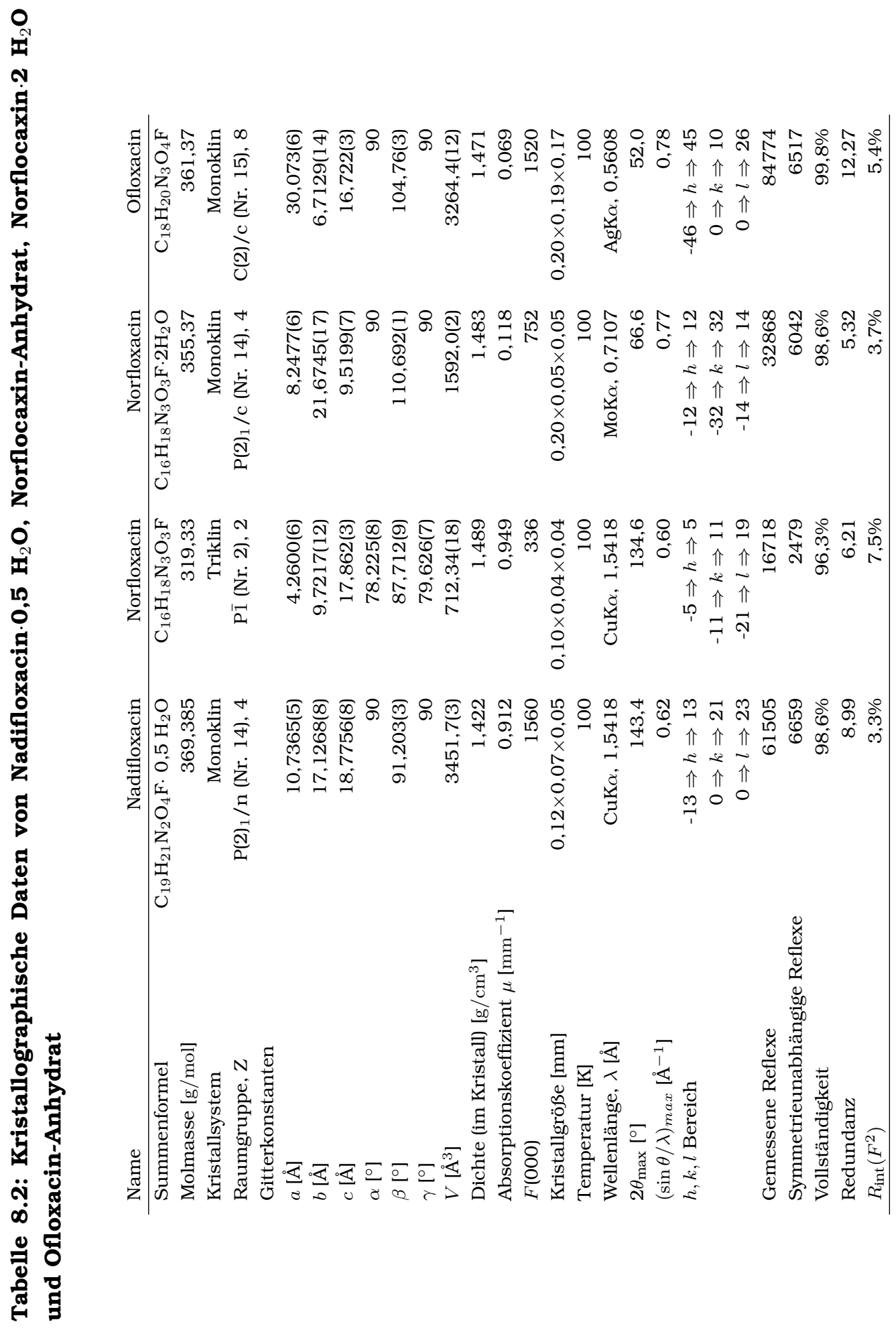




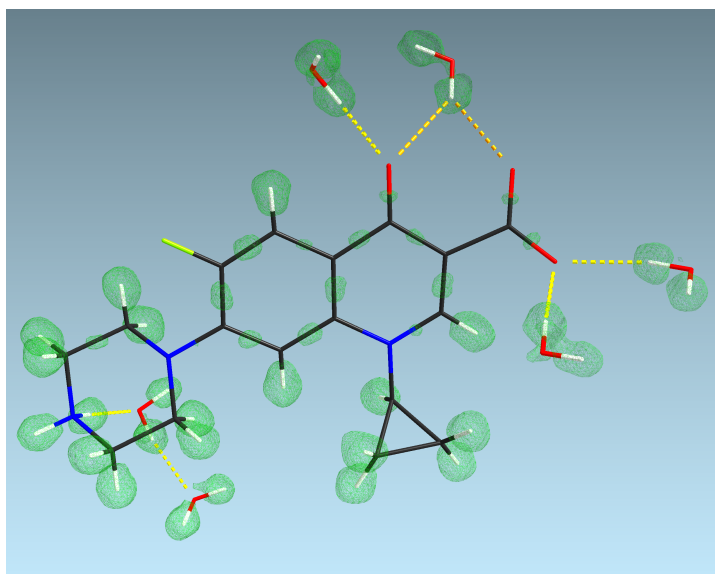

(a) Ciprofloxacin $6 \mathrm{H}_{2} \mathrm{O}$

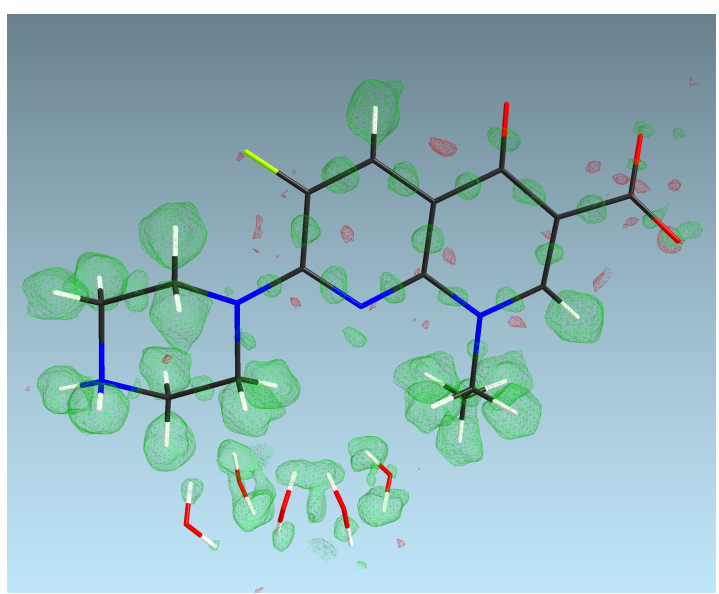

(c) Enoxacin $1,7 \mathrm{H}_{2} \mathrm{O}\left(0,3\right.$ und $\left.-0,3 \mathrm{e} / \AA^{3}\right)$

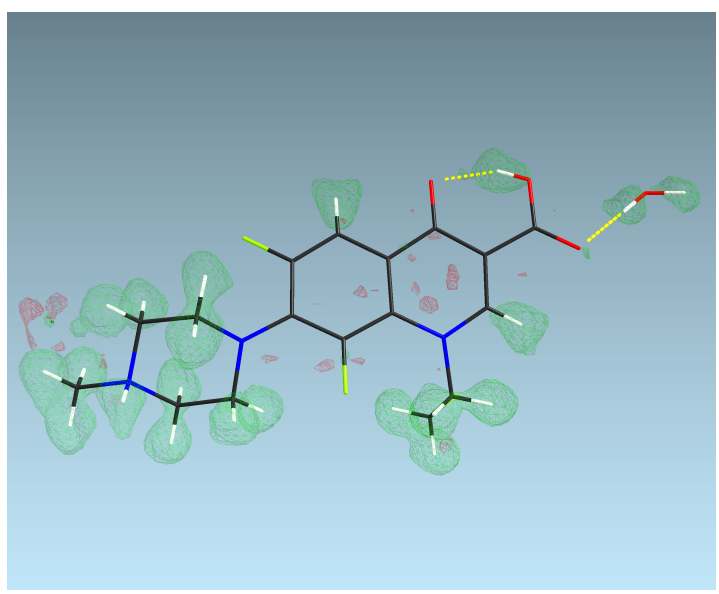

(e) Fleroxacin $\cdot \mathrm{H}_{2} \mathrm{O} \cdot \mathrm{HCl}$

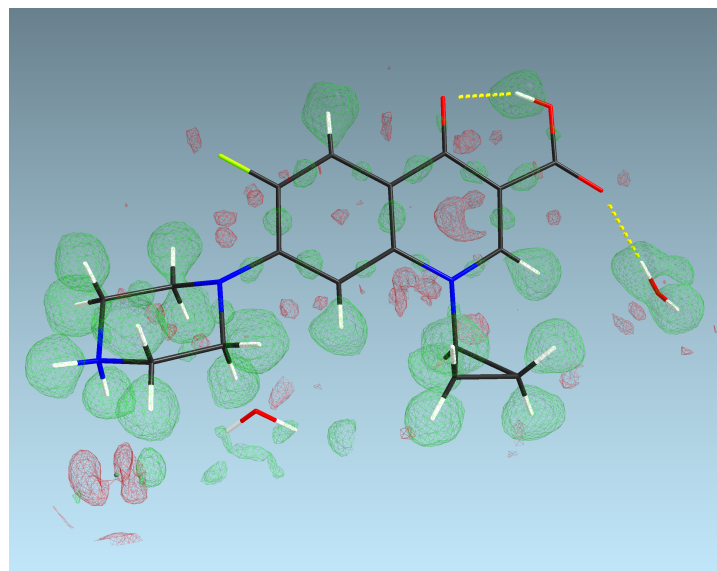

(b) Ciprofloxacin $\cdot 1,4 \mathrm{H}_{2} \mathrm{O} \cdot \mathrm{HCl}$

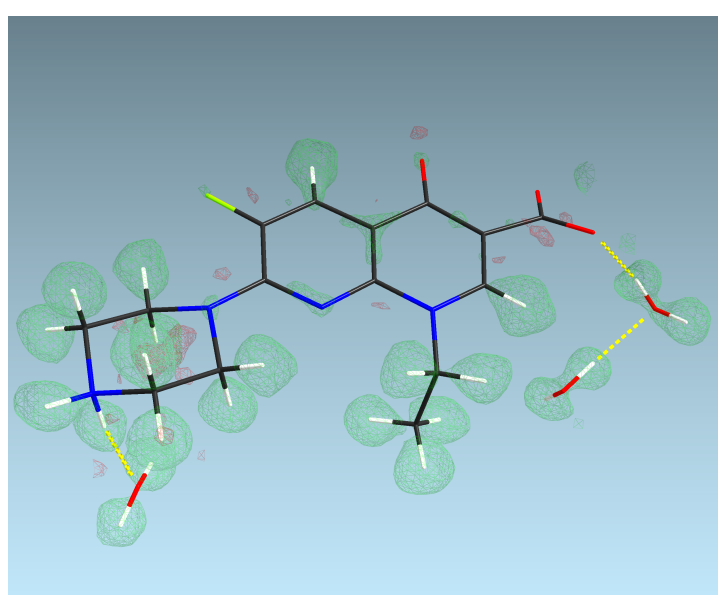

(d) Enoxacin $3 \mathrm{H}_{2} \mathrm{O}$

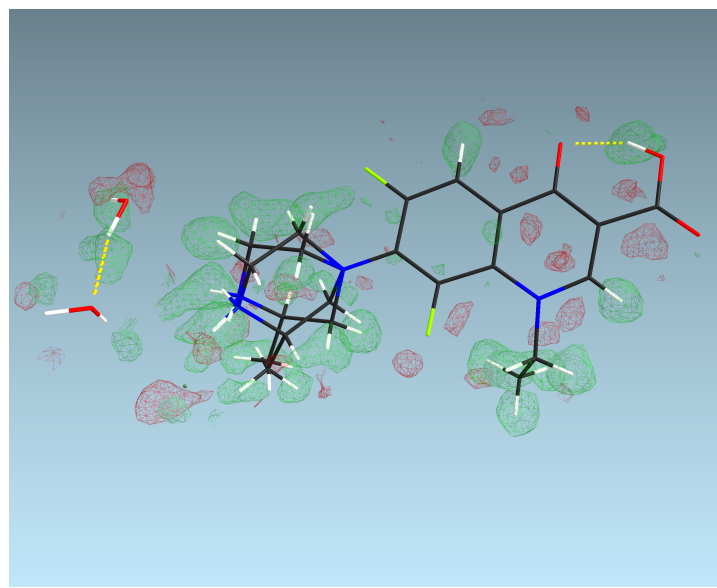

(f) Lomefloxacin $2 \mathrm{H}_{2} \mathrm{O} \cdot 0,95 \mathrm{Cl}^{-}$

Abbildung 8.10: Die Restelektronendichte wurde unter Aussparung der Wasserstoffatome berechnet und ist mit Ausnahme von (c) bei Werten von 0,2 und -0,2 e/ $/ \AA^{3}$ mit SHELXLE [89] kartiert. Bei (c) ist nur eines der beiden Moleküle in der asymmetrischen Einheit gezeigt. 


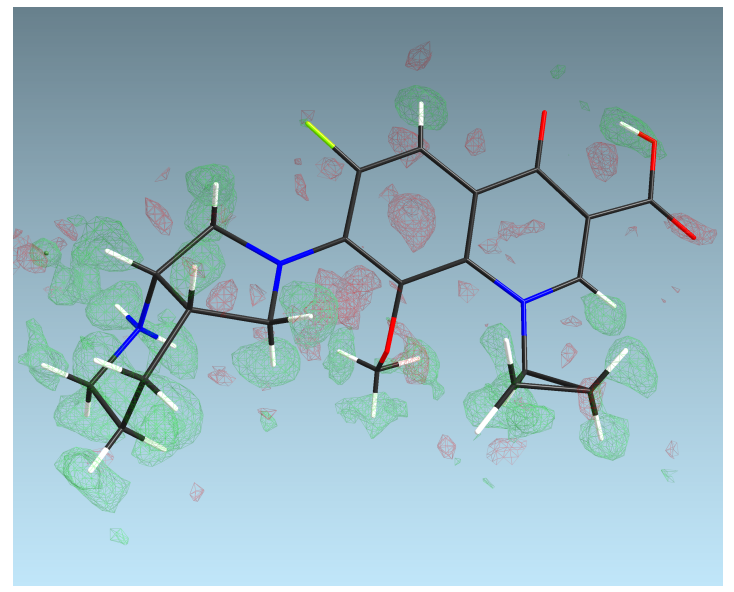

(a) Moxifloxacin $0,5 \mathrm{H}_{2} \mathrm{O} \cdot 0,5 \mathrm{CH}_{3} \mathrm{OH} \cdot \mathrm{HCl}$

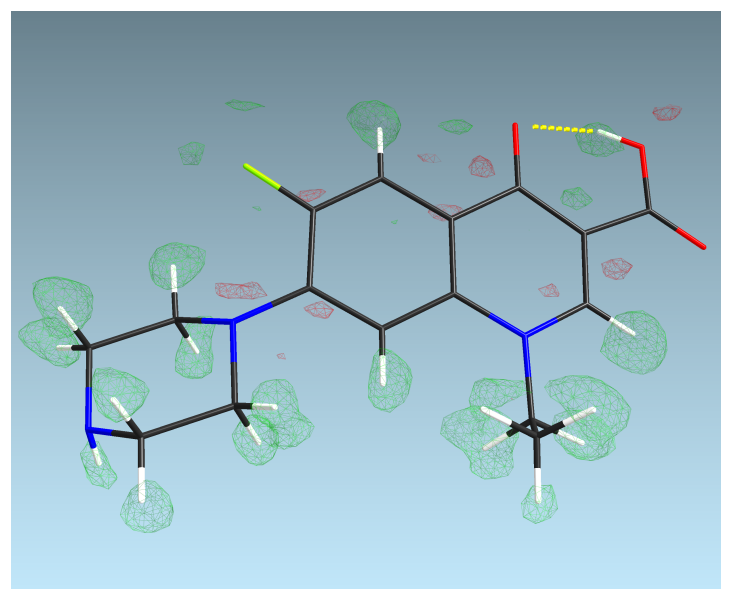

(c) Norfloxacin-Anhydrat

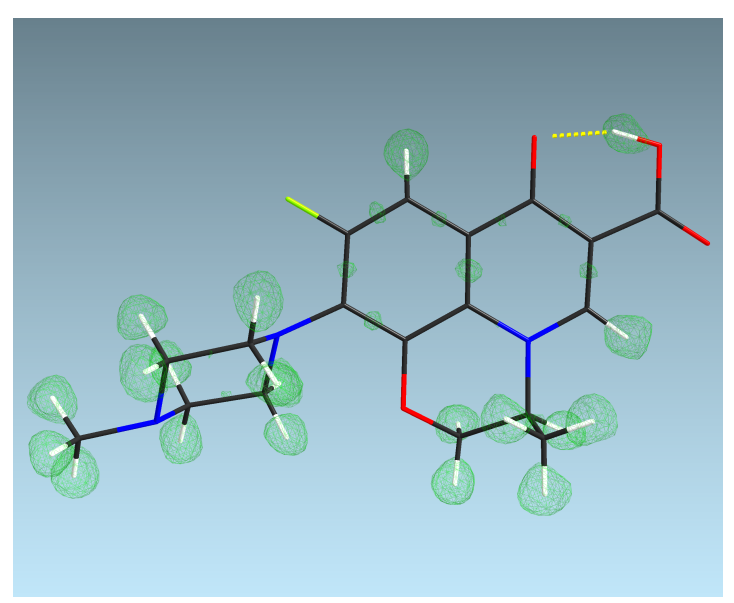

(e) Ofloxacin-Anhydrat

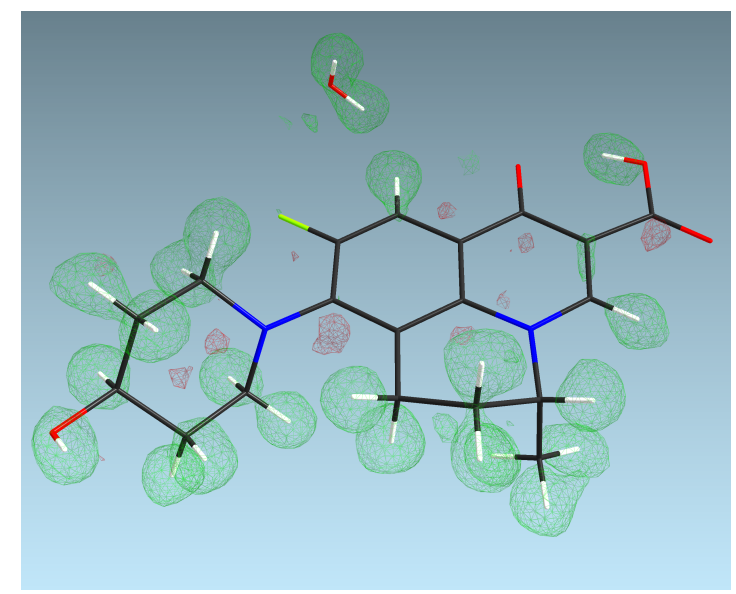

(b) Nadifloxacin $0,5 \mathrm{H}_{2} \mathrm{O}$

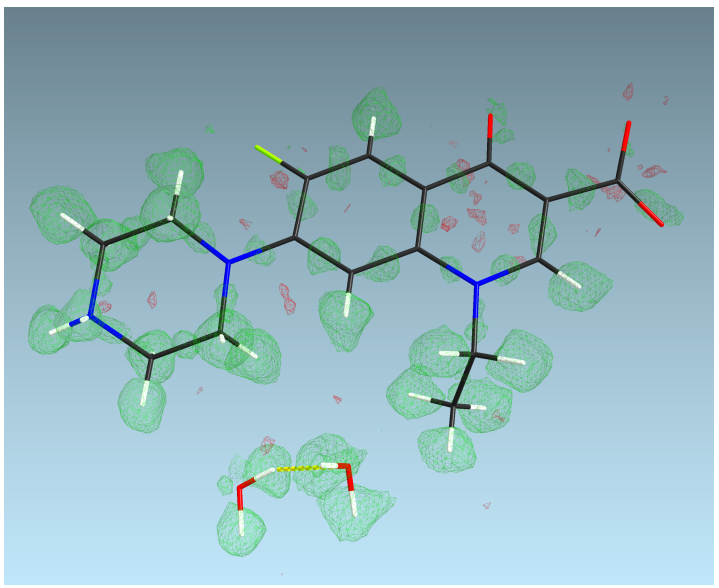

(d) Norfloxacin $2 \mathrm{H}_{2} \mathrm{O}\left(0,3\right.$ und $\left.-0,3 \mathrm{e} / \AA^{3}\right)$

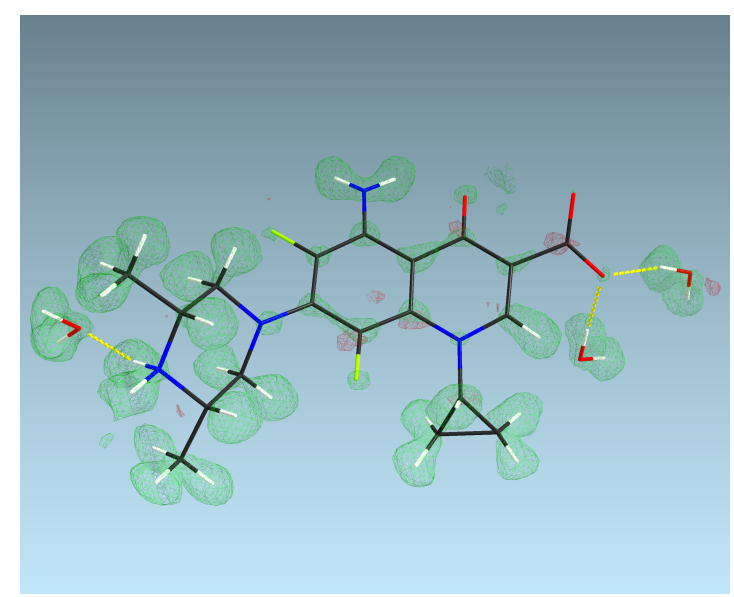

(f) Sparfloxacin $3 \mathrm{H}_{2} \mathrm{O}$

Abbildung 8.11: Die Restelektronendichte ist unter Aussparung der Wasserstoffatome berechnet und ist mit Ausnahme von (d) bei Werten von 0,2 und -0,2 e/ $\AA^{3}$ mit SHELXLE [89] kartiert. Bei (a) und (b) wurde nur eines der beiden Moleküle in der asymmetrischen Einheit gezeigt. 


\subsection{Verifizierung der Modellierung}

\subsubsection{Omit maps und Protonierungszustände}

Zur Verifizierung der Wasserstoffatompositionen wurde die Restelektronendichtekarte des Promolekülmodells unter Aussparung der Wasserstoffatome berechnet. Sie ist in den Abbildungen 8.10 und 8.11 mit SHELXLE [89] bei Werten von $\pm 0,2$ bzw. $\pm 0,3$ e $/ \AA^{3}$ um die Moleküle eingezeichnet. Von Enoxacin 3 $\mathrm{H}_{2} \mathrm{O}$, Moxifloxacin $\cdot 0,5 \mathrm{H}_{2} \mathrm{O} \cdot 0,5 \mathrm{CH}_{3} \mathrm{OH} \cdot \mathrm{HCl}$ und Nadifloxacin $\cdot 0,5 \mathrm{H}_{2} \mathrm{O}$ wurde aus Gründen der Übersichtlichkeit nur eines der beiden Moleküle der asymmetrischen Einheit eingezeichnet. Dass die Wasserstoffatome in allen Strukturen von grünen Restelektronendichtegittern umgeben sind, zeigt, dass die Positionen der Wasserstoffatome experimentell beobachtet wurden. Dies ist aufgrund des geringen Streubeitrags nicht selbstverständlich. Der Protonierungszustand der Moleküle im Festkörper konnte dadurch sicher bestimmt werden. Besonders interessant sind hierbei die unterschiedlichen Solvate der gleichen Fluorochinolone. Während Ciprofloxacin $1,4 \mathrm{H}_{2} \mathrm{O} \cdot \mathrm{HCl}$ als Kation vorliegt, handelt es sich bei bei Ciprofloxacin $6 \mathrm{H}_{2} \mathrm{O}$ um ein Zwitterion. Die beiden Enoxacin-Strukturen $\left(1,7 \mathrm{H}_{2} \mathrm{O}\right.$ und $3 \mathrm{H}_{2} \mathrm{O}$ ) liegen zwitterionisch vor. NorflocaxinAnhydrat liegt ungeladen im Kristall vor, während Norflocaxin $2 \mathrm{H}_{2} \mathrm{O}$ ebenfalls zwitterionisch ist. Es konnte damit gezeigt werden, dass der Protonierungszustand direkt vom jeweiligen Solvat abhängt. Die Anhydrate und das Hemisolvat von Nadifloxacin liegen ungeladen vor, die Hydrochloride als Kationen und die Solvate als Zwitterionen.

\subsubsection{Gütefaktoren}

In den Tabellen 8.3 bis 8.8 werden die Gütefaktoren für die sphärische Verfeinerung in SHELXL (SHELX), der sphärischen Verfeinerung in XD ( $\left.\mathrm{XD}_{\mathrm{IAM}}\right)$ und der Invariom-Verfeinerung $\left(\mathrm{XD}_{\mathrm{INV}}\right)$ gegenüber gestellt. Es zeigt sich, dass die Verwendung asphärischer Atomformfaktoren der Invariom-Datenbank bei elf der zwölf Strukturen zu eine Verbesserung der Gütefaktoren führt. Der $R(F)$ der verbessert sich dabei in Abhängigkeit der Datenqualität um bis zu 1,1\% im Fall von Ciprofloxacin $6 \mathrm{H}_{2} \mathrm{O}$. Bei der fehlgeordneten Lomefloxacin. $2 \mathrm{H}_{2} \mathrm{O} \cdot 0,95$ $\mathrm{Cl}^{-}$Struktur verschlechtert er sich hingegen geringfügig um 0,09\%. Der Unterschied zwischen der SHELX und der $\mathrm{XD}_{\mathrm{IAM}}$ Verfeinerung resultiert aus den verschiedenen Kriterien für beobachtete Reflexe in XD, $I(\vec{h})>3 \sigma[I(\vec{h})]$. Dadurch ist der $R(F)$ bei $\mathrm{XD}_{\text {IAM }}$ in allen Datensätzen niedriger und der $R_{\text {all }}(F)$ 
höher als in SHELX. Wenn in den Verfeinerungen über $1 / \sigma^{2}\left[F_{o}^{2}(\vec{h})\right]$ gewichtet wurde, ist bei den Gewichtungsparametern $\mathbf{a}$ und $\mathbf{b}, 0,0 “$ angegeben.

\begin{tabular}{|c|c|c|c|c|c|c|c|}
\hline \multirow{6}{*}{ 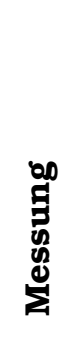 } & \multirow[b]{2}{*}{ Lösungsmittel } & \multicolumn{6}{|c|}{ Ciprofloxacin } \\
\hline & & \multicolumn{3}{|c|}{$6 \mathrm{H}_{2} \mathrm{O}$} & \multicolumn{3}{|c|}{$1,4 \mathrm{H}_{2} \mathrm{O} \mathrm{HCl}$} \\
\hline & Raumgruppe & \multicolumn{3}{|c|}{$\mathrm{P} \overline{1}$} & \multicolumn{3}{|c|}{$\mathrm{P}(2)_{1} / \mathrm{c}$} \\
\hline & Temperatur & \multicolumn{3}{|c|}{$100 \mathrm{~K}$} & \multicolumn{3}{|c|}{$100 \mathrm{~K}$} \\
\hline & $\lambda[\AA ̊]$ & \multicolumn{3}{|c|}{0,71073} & \multicolumn{3}{|c|}{0,7660} \\
\hline & $\sin \theta_{\max } / \lambda\left[\AA^{-1}\right]$ & \multicolumn{3}{|c|}{1,16} & \multicolumn{3}{|c|}{0,78} \\
\hline \multirow{8}{*}{ 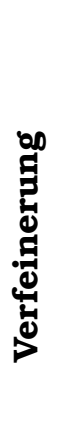 } & $\left.d_{\max }[\AA]\right]$ & \multicolumn{3}{|c|}{0,43} & \multicolumn{3}{|c|}{0,64} \\
\hline & & SHELX & $\mathrm{XD}_{\mathrm{IAM}}$ & $\mathrm{XD}_{\text {INV }}$ & SHELX & $\mathrm{XD}_{\text {IAM }}$ & $\mathrm{XD}_{\text {INV }}$ \\
\hline & Gewicht a & 0,0580 & 0,0580 & 0,0 & 0,0564 & 0,0564 & 0,001 \\
\hline & Gewicht $\mathbf{b}$ & 0,0743 & 0,0743 & 0,0 & 1,5709 & 1,5709 & 1,50 \\
\hline & $R(F)$ & 0,0313 & 0,0315 & 0,0205 & 0,0427 & 0,0420 & 0,0339 \\
\hline & $R_{\text {all }}(F)$ & 0,0391 & 0,0462 & 0,0352 & 0,0463 & 0,0473 & 0,0392 \\
\hline & $S$ Goof & 1,064 & 1,2705 & 2,5770 & 0,926 & 1,0076 & 0,9541 \\
\hline & $\Delta \rho_{\max }-\Delta \rho_{\min }$ & 0,96 & 1,08 & 0,72 & 1,64 & 1,58 & 1,57 \\
\hline
\end{tabular}

Tabelle 8.3: Messung und Verfeinerung von Ciprofloxacin $6 \mathrm{H}_{2} \mathrm{O}$ und Ciprofloxacin $1,4 \mathrm{H}_{2} \mathrm{O} \cdot \mathrm{HCl}$

\section{Enoxacin}

\begin{tabular}{|c|c|c|c|c|c|c|c|}
\hline \multirow{5}{*}{ 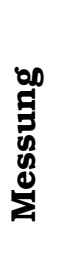 } & Lösungsmittel & \multicolumn{3}{|c|}{$1,7 \mathrm{H}_{2} \mathrm{O}$} & \multicolumn{3}{|c|}{$3 \mathrm{H}_{2} \mathrm{O}$} \\
\hline & Raumgruppe & \multicolumn{3}{|c|}{$\mathrm{P} 2_{1} / \mathrm{c}$} & \multicolumn{3}{|c|}{$\mathrm{P}(2)_{1} / \mathrm{c}$} \\
\hline & Temperatur & \multicolumn{3}{|c|}{$100 \mathrm{~K}$} & \multicolumn{3}{|c|}{$100 \mathrm{~K}$} \\
\hline & $\lambda[\AA]$ & \multicolumn{3}{|c|}{0,71073} & \multicolumn{3}{|c|}{0,71073} \\
\hline & $\sin \theta_{\max } / \lambda\left[\AA^{-1}\right]$ & \multicolumn{3}{|c|}{1,02} & \multicolumn{3}{|c|}{0,65} \\
\hline \multirow{8}{*}{ 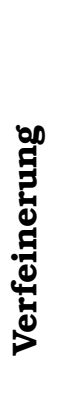 } & $d_{\max }[\AA ̊ \Omega]$ & \multicolumn{3}{|c|}{0,49} & \multicolumn{3}{|c|}{0,77} \\
\hline & & SHELX & $\mathrm{XD}_{\text {IAM }}$ & $\mathrm{XD}_{\text {INV }}$ & SHELX & $\mathrm{XD}_{\text {IAM }}$ & $\mathrm{XD}_{\text {INV }}$ \\
\hline & Gewicht a & 0,0594 & 0,0594 & 0,0 & 0,0526 & 0,0526 & 0,0400 \\
\hline & Gewicht b & 0,4430 & 0,4430 & 0,0 & 0,3952 & 0,3952 & 0,0001 \\
\hline & $R(F)$ & 0,0414 & 0,0376 & 0,0276 & 0,0369 & 0,0346 & 0,0256 \\
\hline & $R_{\text {all }}(F)$ & 0,0704 & 0,0873 & 0,0782 & 0,0518 & 0,0589 & 0,0500 \\
\hline & $S$ Goof & 1,077 & 1,3574 & 2,8305 & 1,014 & 1,0740 & 1,0829 \\
\hline & $\Delta \rho_{\max }-\Delta \rho_{\min }$ & 1,21 & 1,07 & 0,65 & 0,49 & 0,51 & 0,30 \\
\hline
\end{tabular}

Tabelle 8.4: Messung und Verfeinerung von Enoxacin $1,7 \quad \mathrm{H}_{2} \mathrm{O}$ und Enoxacin $3 \mathrm{H}_{2} \mathrm{O}$ 


\begin{tabular}{|c|c|c|c|c|c|c|}
\hline & \multicolumn{3}{|c|}{ Fleroxacin } & \multicolumn{3}{|c|}{ Lomefloxacin } \\
\hline Lösungsmittel & \multicolumn{3}{|c|}{$\mathrm{H}_{2} \mathrm{O} \mathrm{HCl}$} & \multicolumn{3}{|c|}{$2 \mathrm{H}_{2} \mathrm{O} 0,95 \mathrm{Cl}$} \\
\hline Raumgruppe & \multicolumn{3}{|c|}{$\mathrm{P} 2_{1} / \mathrm{c}$} & \multicolumn{3}{|c|}{$\mathrm{P} 22_{1} / \mathrm{c}$} \\
\hline Temperatur & \multicolumn{3}{|c|}{298 K } & \multicolumn{3}{|c|}{$100 \mathrm{~K}$} \\
\hline$\lambda[\AA]$ & \multicolumn{3}{|c|}{0,71073} & \multicolumn{3}{|c|}{0,80000} \\
\hline $\sin \theta_{\max } / \lambda\left[\AA^{-1}\right]$ & \multicolumn{3}{|c|}{0,60} & \multicolumn{3}{|c|}{0,54} \\
\hline \multirow[t]{2}{*}{$d_{\max }[\AA ̊]$} & \multicolumn{3}{|c|}{0,84} & \multicolumn{3}{|c|}{0,92} \\
\hline & SHELX & $\mathrm{XD}_{\mathrm{IAM}}$ & $\mathrm{XD}_{\text {INV }}$ & SHELX & XDIAM & $\mathrm{XD}_{\text {INV }}$ \\
\hline Gewicht a & 0,0585 & 0,0585 & 0,04 & 0,1124 & 0,0 & 0,0 \\
\hline Gewicht b & 0,00 & 0,00 & 0,01 & 3,98 & 0,0 & 0,0 \\
\hline$R(F)$ & 0,0380 & 0,0343 & 0,0280 & 0,0706 & 0,0694 & 0,0703 \\
\hline$R_{\text {all }}(F)$ & 0,0669 & 0,0818 & 0,0756 & 0,0723 & 0,0722 & 0,0737 \\
\hline$S$ Goof & 0,956 & 1,0105 & 0,9934 & 1,310 & 3,802 & 3,910 \\
\hline$\Delta \rho_{\max }-\Delta \rho_{\min }$ & 0,54 & 0,48 & 0,53 & 0,81 & 0,85 & 0,76 \\
\hline
\end{tabular}

Tabelle 8.5: Messung und Verfeinerung von Fleroxacin $\cdot \mathrm{H}_{2} \mathrm{O} \mathrm{HCl}$ und Lomefloxacin $\cdot 2 \mathrm{H}_{2} \mathrm{O} 0,95 \cdot \mathrm{Cl}^{-}$

\section{Norfloxacin}

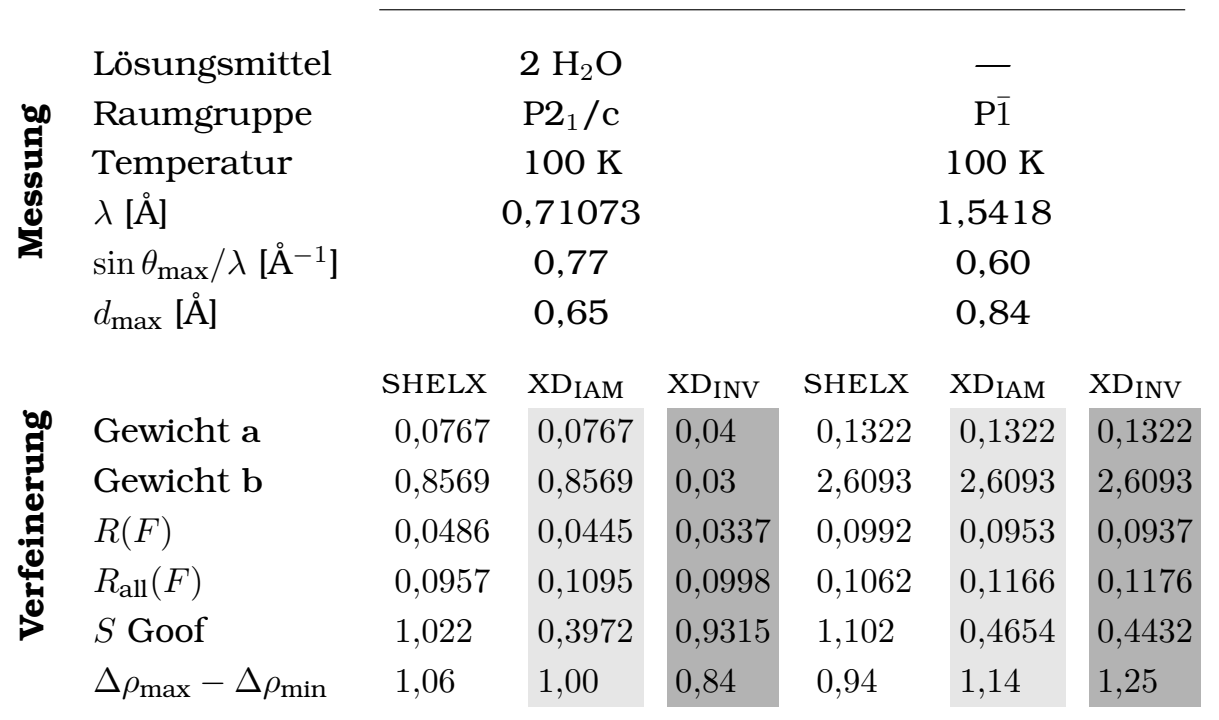

Tabelle 8.6: Messung und Verfeinerung von Norflocaxin $2 \mathrm{H}_{2} \mathrm{O}$ und Norflocaxin-Anhydrat 
Sparfloxacin

Lösungsmittel

䏛

of Raumgruppe

Temperatur

$\lambda[\AA ̊]$

$\sin \theta_{\max } / \lambda\left[\AA^{-1}\right]$

$d_{\max }[\AA]$

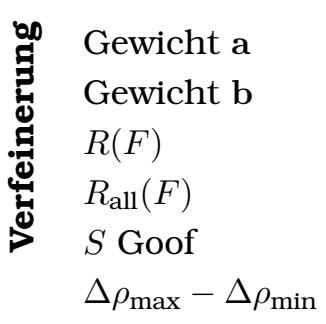

\begin{tabular}{ccl}
\multicolumn{3}{c}{ Sparfloxacin } \\
\hline \multicolumn{3}{c}{$3 \mathrm{H}_{2} \mathrm{O}$} \\
$\mathrm{P}_{1} / \mathrm{n}$ \\
$298 \mathrm{~K}$ \\
1,5418 \\
& 0,61 \\
& 0,82 \\
SHELX & $\mathrm{XD}_{\text {IAM }}$ & $\mathrm{XD}_{\text {INV }}$ \\
0,0394 & 0,0394 & 0,001 \\
1,1263 & 1,1263 & 0,40 \\
0,0517 & 0,0537 & 0,0478 \\
0,0615 & 0,0617 & 0,0562 \\
1,045 & 1,0954 & 1,3203 \\
0,50 & 0,54 & 0,48
\end{tabular}

Moxifloxacin

$\mathrm{HCl} \mathrm{0,5} \mathrm{H}_{2} \mathrm{O} 0,5 \mathrm{CH}_{3} \mathrm{OH}$

$\mathrm{P} 2$

$273 \mathrm{~K}$

0,7107

0,60

0,84

SHELX XD IAM $\quad \mathrm{XD}_{\text {INV }}$

$\begin{array}{lll}0,0417 & 0,0417 & 0,03\end{array}$

$\begin{array}{lll}0,0 & 0,0 & 0,0\end{array}$

$0,0472 \quad 0,0389$

$0,0815 \quad 0,0868$

$0,863 \quad 0,9117$

$0,47 \quad 0,45$

0,0375

0,0872

1,0154

0,44

Tabelle 8.7: Messung und Verfeinerung von Sparfloxacin $3 \mathrm{H}_{2} \mathrm{O}$ und Moxifloxacin $\cdot 0,5 \mathrm{H}_{2} \mathrm{O} \cdot 0,5 \mathrm{CH}_{3} \mathrm{OH} \cdot \mathrm{HCl}$

Lösungsmittel

a Raumgruppe

Temperatur

$\lambda[\AA]$

$\sin \theta_{\max } / \lambda\left[\AA^{-1}\right]$

$d_{\max }[\AA]$

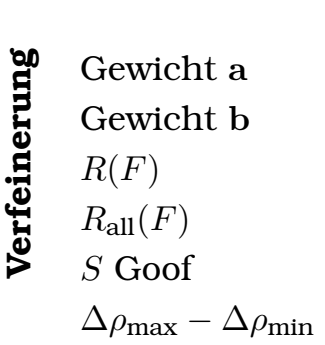

\section{Nadifloxacin}

$$
0,5 \mathrm{H}_{2} \mathrm{O}
$$

$\mathrm{P} 2{ }_{1} / \mathrm{n}$

$100 \mathrm{~K}$

1,5418

0,61577

0,81199

$\begin{array}{cll}\text { SHELX } & \text { XD }_{\text {IAM }} & \text { XD INV }^{\text {INV }} \\ 0,0818 & 0,0818 & 0,08 \\ 2,9411 & 2,9411 & 1,37 \\ 0,0572 & 0,0571 & 0,0536 \\ 0,0658 & 0,0773 & 0,0747 \\ 1,054 & 0,3517 & 0,4652 \\ 1,32 & 1,34 & 1,42\end{array}$

SHELX

0,0722

3,3730

0,0451

0,0594

1,016

$1,048 \quad 1,08$
$100 \mathrm{~K}$

0,5608

0,78125

0,64000

Ofloxacin

\begin{tabular}{|l|l}
\hline $\mathrm{XD}_{\text {IAM }}$ & $\mathrm{XD}_{\text {INV }}$ \\
0,0722 & 0,0 \\
3,3730 & 0,0 \\
0,0458 & 0,0346 \\
0,0810 & 0,0701 \\
0,7823 & 2,7286 \\
1,08 & 0,72 \\
\hline
\end{tabular}

Tabelle 8.8: Messung und Verfeinerung von Nadifloxacin $0,5 \mathrm{H}_{2} \mathrm{O}$ und Ofloxacin-Anhydrat 


\subsection{Elektrostatisches Potential}

Die Berechnung des elektrostatischen Potentials erfolgt nach der Methode von Volkov et. al [58] unmittelbar aus der dreidimensionalen Elektronendichteverteilung des Invariom-Modells mit dem Programmteil XDPROP des XD Programmpakets [46]. Dabei wurden Lösungsmittelmoleküle und $\mathrm{Cl}^{-}$-Gegenionen nicht mit einbezogen. Im Fall von zwei Fluorochinolonmolekülen in der asymmetrischen Einheit (Enoxacin· 1,7 $\mathrm{H}_{2} \mathrm{O}$, Moxifloxacin·0,5 $\mathrm{H}_{2} \mathrm{O} \cdot 0,5 \mathrm{CH}_{3} \mathrm{OH} \cdot \mathrm{HCl}$ und Nadifloxacin $0,5 \mathrm{H}_{2} \mathrm{O}$ ) erfolgten die Berechnungen nur für eines der Moleküle. Bei der fehlgeordneten Struktur von Lomefloxacin $2 \mathrm{H}_{2} \mathrm{O} 0,95 \mathrm{Cl}$ wurde das höher populierte Molekül zur Berechnung der elektrostatischen Eigenschaften verwendet. In den Abbildungen 8.12, 8.13 und 8.14 sind die elektrostatischen Potentiale der zwölf Fluorochinolone auf der Van-der-Waals-Oberfläche bei einer Elektronendichte von $\rho=0,0067$ e $/ \AA^{3}$ bzw. $\rho=0,001$ au mit dem Programm MolecoolQT [72] als Farbcode aufgetragen. Zusätzlich ist der Potentialverlauf durch Konturlinien auf der Oberfläche verdeutlicht.

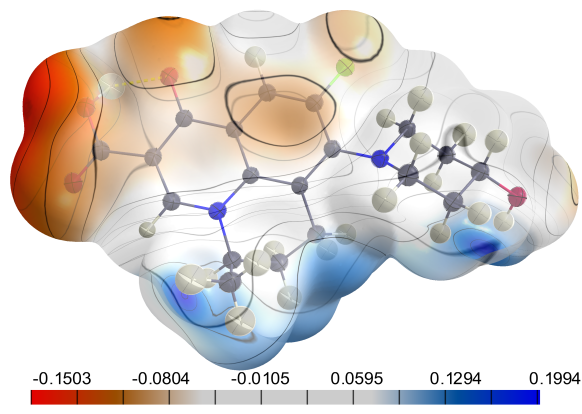

(a) Nadifloxacin $0,5 \mathrm{H}_{2} \mathrm{O}$

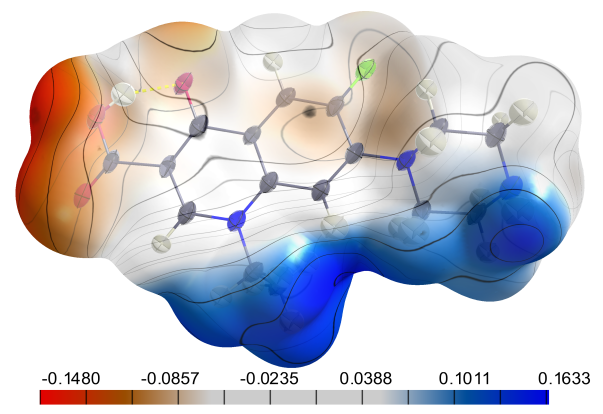

(b) Norflocaxin-Anhydrat

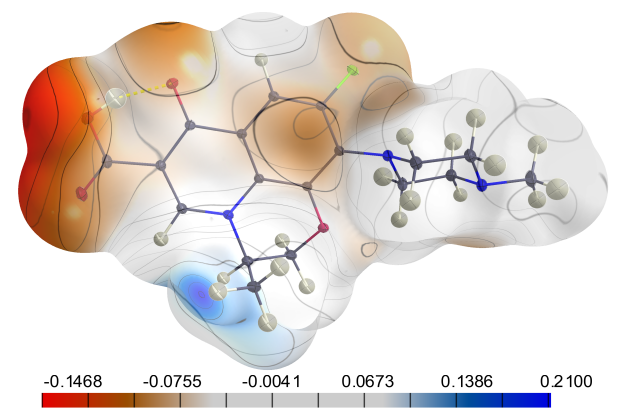

(c) Ofloxacin-Anhydrat

Abbildung 8.12: Elektrostatisches Potential der ungeladenen Fluorochinolone. Die Werte der Legenden sind in e/Å angegeben. 
Bei den ungeladenen Molekülen liegt das elektrostatische Potential zwischen $-0,15$ und 0,21 e/Å. Im Fall der kationischen Moleküle liegt es zwischen -0,18 und 0,30 e/Å und bei den zwitterionischen Molekülen zwischen -0,31 und $0,37 \mathbf{e} / \AA$.

Die Carboxylat bzw. Carbonsäure-Gruppen und der bei den Kationen und Zwitterionen protonierte Piperazin-Ring dominieren die elektrostatischen Strukturmerkmale, während die unterschiedlichen Substitutionsmuster der verschiedenen Fluorochinolone kaum einen Unterschied machen. Es gibt insgesamt eine sehr gute Übereinstimmung des ESP der verschiedenen Strukturen mit gleichem Protonierungszustand. Dies bestätigt die in der Wirkstoffforschung verbreitete Hypothese, dass Moleküle mit gleicher Wirksamkeit neben einer ähnlichen Konformation auch ähnliche elektrostatische Eigenschaften aufweisen. Die Varianz der Protonierungszustände könnte auch der Grund für die gute Bioverfügbarkeit der Moleküle sein, da sie sich protischen und aprotischen Umgebungen anpassen können.

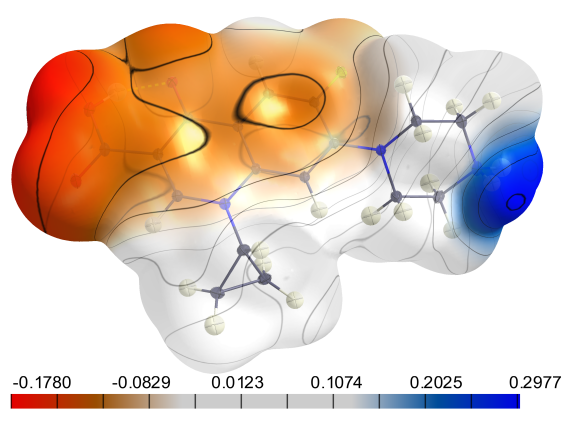

(a) Ciprofloxacin $\cdot 1,4 \mathrm{H}_{2} \mathrm{O} \cdot \mathrm{HCl}$

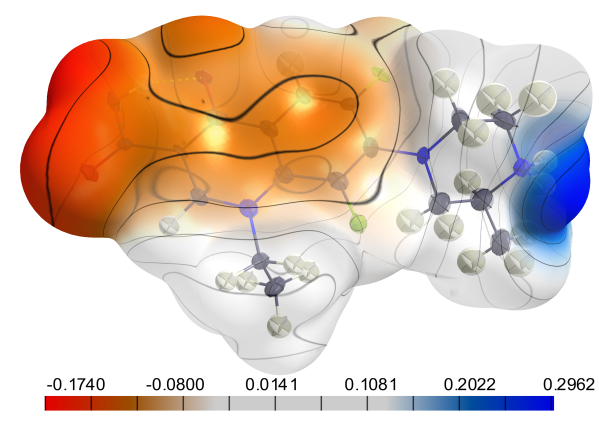

(c) Lomefloxacin $2 \mathrm{H}_{2} \mathrm{O} \cdot 0,95 \mathrm{Cl}^{-}$

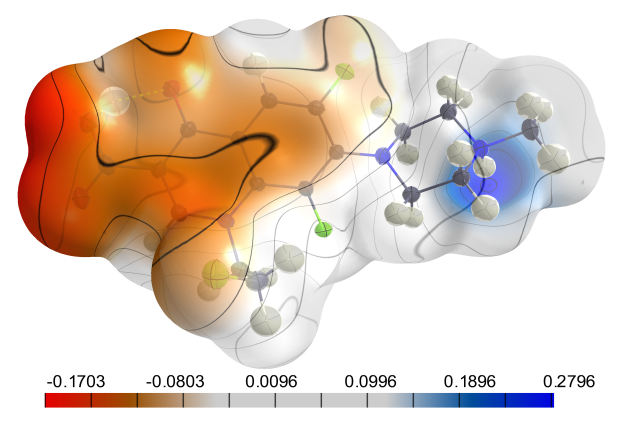

(b) Fleroxacin $\cdot \mathrm{H}_{2} \mathrm{O} \cdot \mathrm{HCl}$

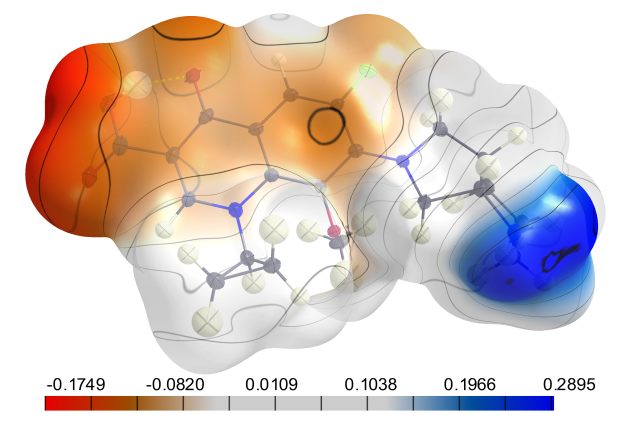

(d) Moxifloxacin $\cdot 0,5 \mathrm{H}_{2} \mathrm{O} \cdot 0,5 \mathrm{CH}_{3} \mathrm{OH} \cdot \mathrm{HCl}$

Abbildung 8.13: Elektrostatisches Potential der kationischen Fluorochinolone. Die Werte der Legenden sind in e/Å angegeben. 


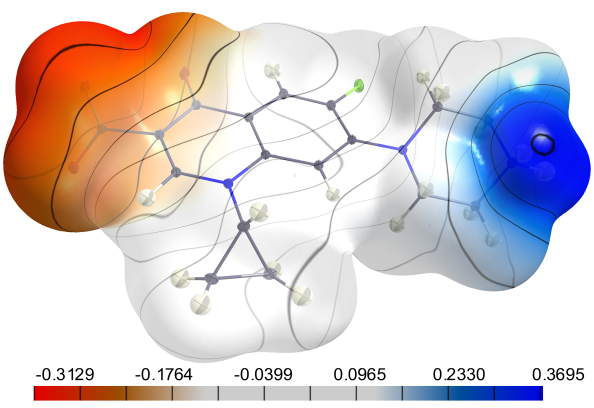

(a) Ciprofloxacin $6 \mathrm{H}_{2} \mathrm{O}$

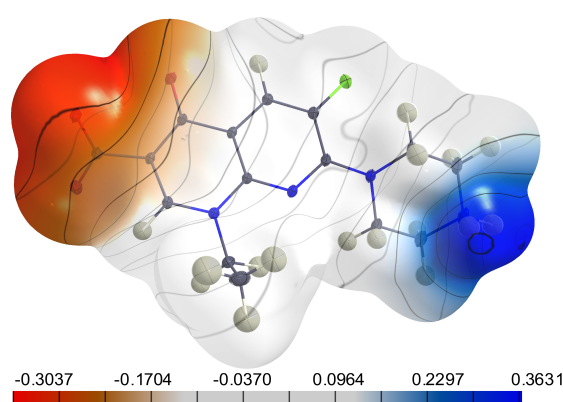

(c) Enoxacin $3 \mathrm{H}_{2} \mathrm{O}$

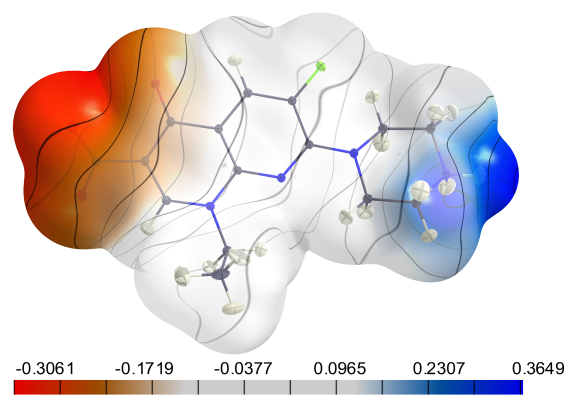

(b) Enoxacin $1,7 \mathrm{H}_{2} \mathrm{O}$

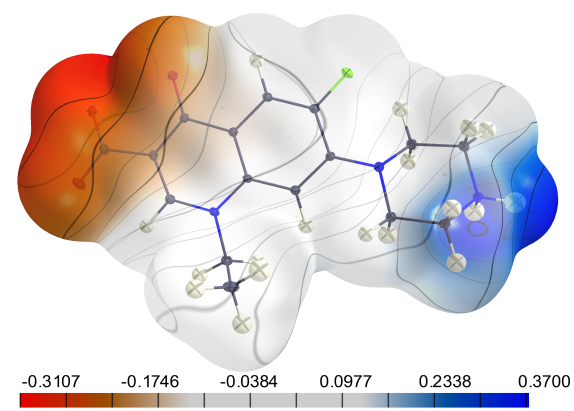

(d) Norflocaxin·2 $\mathrm{H}_{2} \mathrm{O}$

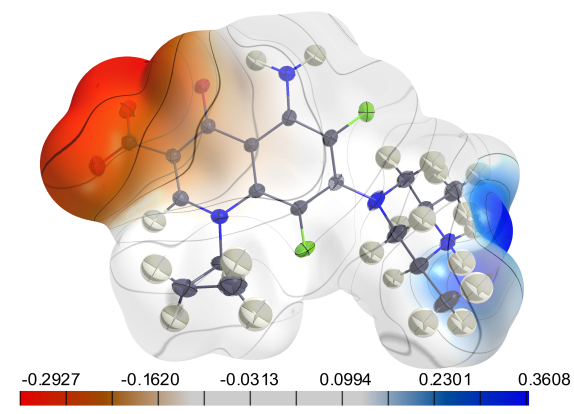

(e) Sparfloxacin $3 \mathrm{H}_{2} \mathrm{O}$

Abbildung 8.14: Elektrostatisches Potential der zwitterionischen Fluorochinolone. Die Werte der Legenden sind in e/Å angegeben.

\subsubsection{Politzer-Analyse}

Neben der optischen Analyse der Oberflächenpotentiale kann auch das Verfahren nach Politzer et al. [63] verwendet werden. Dabei werden Durchschnittswerte vom positiven Potential $\bar{V}_{S}^{+}$, negativen Potential $\bar{V}_{S}^{-}$und vom Gesamtpotential $\bar{V}_{S}$ berechnet. Wie in Abschnitt 3.6.1 auf Seite 32 beschrieben wird 
zusätzlich die Varianz $\sigma_{\text {tot }}^{2}$ und die Standardabweichung des Gesamtpotentials $\Pi$ berechnet. Die Ergebnisse der Analyse sind in Tabelle 8.9 zusammengefasst. Die Werte von $\bar{V}_{S}^{+}, \bar{V}_{S}^{+}$und $\Pi$ zeigen dabei eine deutliche Korrelation mit dem Protonierungszustand der Moleküle.

Durch die leichte Vergleichbarkeit der durchschnittlichen Oberflächenpotentiale sind die Werte der Politzer-Analyse insbesondere auch für HochdurchsatzVerfahren großer Substanzbibliotheken geeignet.

\subsection{Dipolmomente}

Dipolmomente sind im Invariom-Ansatz direkt aus der Verfeinerung (INV) zugänglich. Um die Qualität der erhaltenen Dipolmomente zu überprüfen wurde ausgehend von der experimentellen Geometrie eine Single-Point-Energieminimierung (SP) mit der Basis [B3LYP/D95++3df3pd] durchgeführt. Im Kristall vorhandene Lösungsmittelmoleküle oder Gegenionen $\left(\mathrm{Cl}^{-}\right)$wurden nicht mit berechnet. Zusätzlich wurde eine Multipolprojektion (MP) der Berechnungsergebnissen durchgeführt. Die Dipolmomente der experimentelle Invariom-Verfeinerung wurden mit denen der Single-Point-Energieminimierung und der Multipolprojektion der Wellenfunktion (MP) verglichen.

Das Dipolmoment ist nur im Fall von ungeladenen Entitäten unabhängig vom gewählten Ursprung [117]. Da das Programm GAUSSIAN nicht der Konvention folgt das Massezentrum als Ursprung zu verwenden, werden in Tabelle 8.10 nur die Werte von INV und MP für die kationischen Strukturen angegeben. Durch die Multipolprojektion wird dieses Problem umgangen und in den ungeladenen und zwitterionischen Fällen gleichzeitig der Einfluss des Multipolmodells im Vergleich zur direkten quantenchemischen Berechnung deutlich. Die absolute und relative Abweichung zwischen den Dipolmomenten aus der Invariom-Verfeinerung und der Multipolprojektion sind ebenfalls in Tabelle 8.10 aufgeführt. Im Fall der zwitterionischen Moleküle beträgt die Abweichung bis zu 5\%; für die ungeladenen Moleküle bis zu 10\% und bei den kationischen Molekülen bis zu 20\%. Die kationischen Molekülen haben mit bis zu 5,7 Debye auch die höchste absolute Abweichung im Dipolmoment. Im Hinblick auf die Zeitersparnis bei der Berechnung im Vergleich zu den SinglePoint-Energieberechnungen ist die Genauigkeit des Invariom-Modells zur Berechnung des Dipolmoments klar zu favorisieren. Es ist damit für große Substanzbibliotheken im Hochdurchsatz-Verfahren geeignet. 


\section{Dipolmoment}

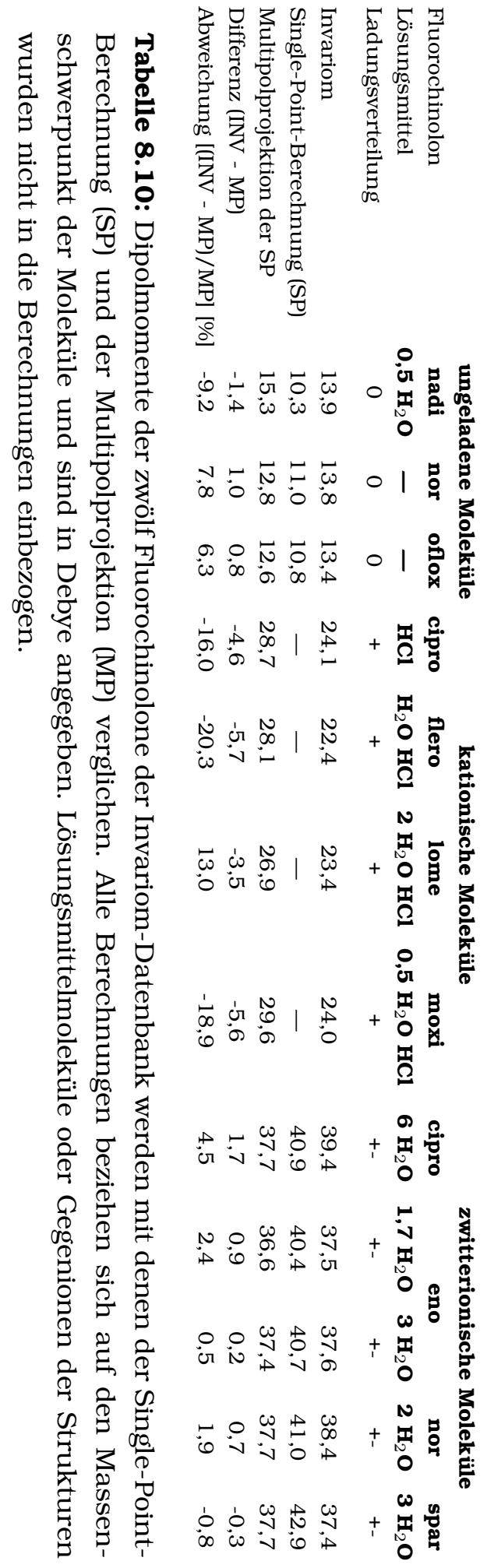

\section{Politzer-Analyse}

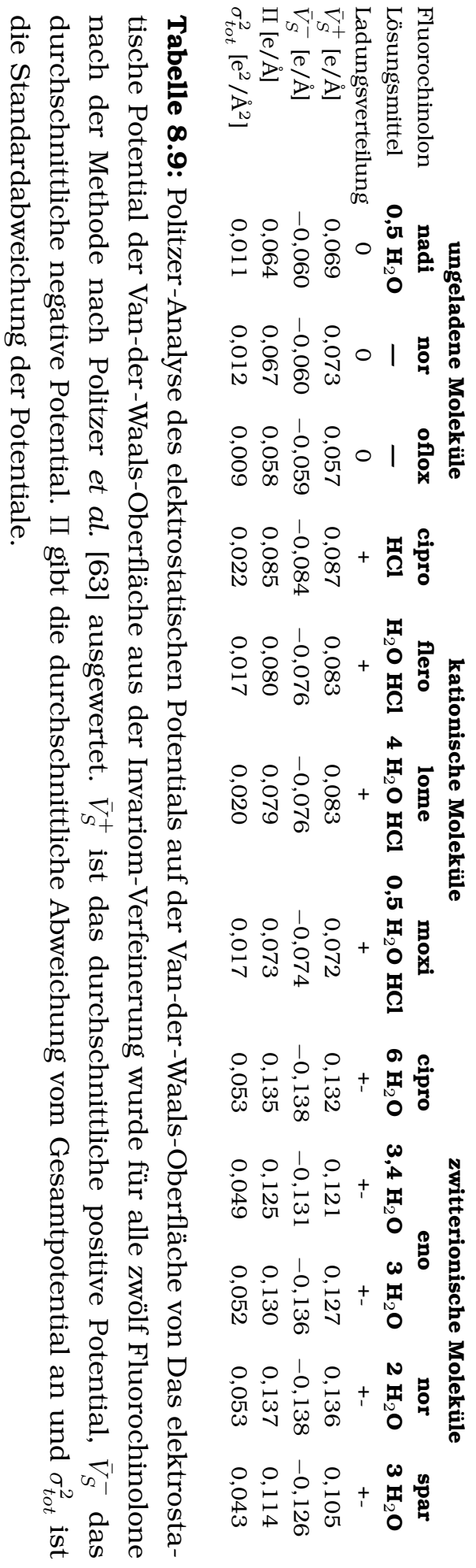




\title{
8.10 Vergleichsprüfung zur Transferierbarkeit
}

Die Gültigkeit der Annahmen zur Rekonstruktion der molekularen Elektronendichte wurden bereits indirekt durch den Vergleich der abgeleiteten Eigenschaften mit den Ergebnissen des Invariom-Transfers gezeigt. Bei der Untersuchung experimenteller Daten der nichtproteinogenen Aminosäuren L-Ornithin [82] und Aminoisobuttersäure [83] sowie beim Makrolidantibiotikum Roxithromycin [66] wurde die Differenzelektronendichte aus der Multipolverfeinerung und den Atomformfaktoren der Invariom-Datenbank bereits erfolgreich angewendet.

\subsubsection{Experimentelle Strukturfaktoren von Enoxacin $1,7 \mathrm{H}_{2} \mathrm{O}$}

Die Elektronendichte der Invariom-Verfeinerung wurde von der Elektronendichte der experimentellen Multipolverfeinerung abgezogen. Dafür wurde die Geometrie der Invariom-Verfeinerung auch für die Multipolverfeinerung verwendet. Die Ergebnisse werden in Abbildung 8.15 gezeigt. Dabei gibt es eine gute Übereinstimmung zwischen der Elektronendichte der Invariom-Datenbank und der Multipolverfeinerung, wobei die Maxima und Minima $\pm 0,2 e / \AA^{3}$ nicht übersteigen.

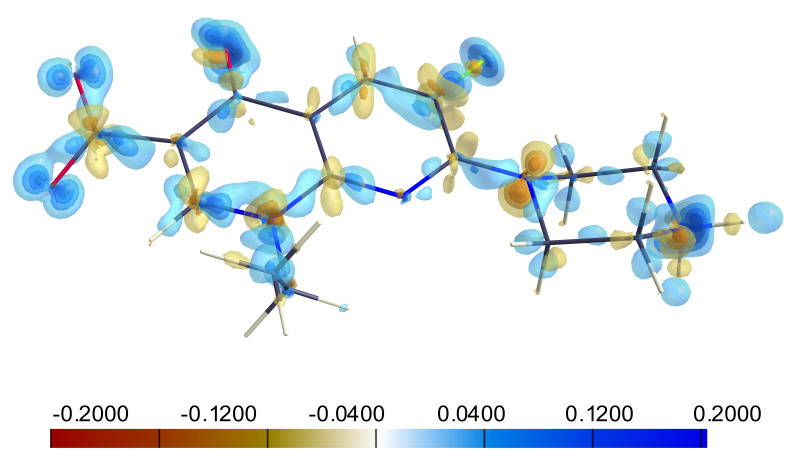

\begin{abstract}
Abbildung 8.15: Modellunterschiede zwischen der experimentellen Multipolverfeinerung (MV) und der Invariom-Verfeinerung (IV) von Enoxacin 1,7 $\mathrm{H}_{2} \mathrm{O}$. Isooberflächen der Differenzelektronendichte MV-INV sind im Bereich von $-0,2$ bis 0,2 e/ $/ \AA^{3}$ und einem Abstand von 0,05 e/ $/ \AA^{3}$ mit MoleCOOLQT [72] eingezeichnet.
\end{abstract}

Die Charakteristika der Differenzelektronendichte sind damit betragsmäßig deutlich kleiner als die der Restelektronendichte von $\pm 0,4 e / \AA^{3}$ und der De- 
formationselektronendichte von $\pm 0,7 e / \AA^{3}$.

\subsubsection{Theoretische Strukturfaktoren der Fluorochinolone}

Durch quantenchemische Geometrieoptimierungen der Fluorochinolone wird die Genauigkeit der Invariom-Näherung zur Rekonstruktion der molekularen Elektronendichte nun erstmals mit dem in Abschnitt 5.7 beschriebenen Verfahren verifiziert. Die quantenchemische Geometrieoptimierung mit der verwendetet Basis [B3LYP/D95++3df3pd] ist sehr zeitaufwendig, auch wenn als Startpunkt die experimentelle Geometrie verwendet wird, da ein hoher Grad an konformationeller Freiheit vorliegt. Besonders im Fall geladener oder zwitterionischer Moleküle war es nicht einfach das globale Energieminimum zu finden.

Die Vergleichsprüfung mit den theoretischen Strukturfaktoren hat den Vorteil, dass dabei experimentelle Fehler ausgeschlossen werden können und auch keine Korrelation von Auslenkungsparametern und Multipolparametern vorliegen kann. Im Idealfall sollte es keine Unterschiede zwischen der Summe der Invariom-Fragmente und der Elektronendichte des gesamten Moleküls aus der Geometrieoptimierung geben.

In den Abbildungen 8.16, 8.17 und 8.18 wird die Differenz der Elektronendichte der Geometrieoptimierung und der Invariom-Elektronendichte für die verschiedenen im Kristall vorhandenen Moleküle und Protonierungszustände gezeigt. Die komplexen Bindungsverältnisse der Fluorochinolone können durch die Elektronendichte-Fragmente der Invariom-Datenbank wie im Fall der experimentellen Strukturfaktoren mit einer Genauigkeit von von $\pm 0,2$ e $/ \AA^{3}$ reproduziert werden. Die höchsten Abweichungen treten dabei an Sauerstoffatomen der Carbonsäure auf. Als Ursache kommen dabei folgende Punkte in Betracht:

- Intramolekulare Wasserstoffbrückenbindungen im Fall der ungeladenen und kationischen Moleküle

- Die Invariom-Näherung für einige Sauerstoffatome, für die ausschließlich nächste Nachbarn zur Ableitung der Modellverbindungen betrachten werden.

- Bekannte Schwachstellen des Multipolmodells um Feinheiten der Elektronendichte von Sauerstoffatomen abzubilden [118, 119, 120, 121]. 
Die erreichte Genauigkeit liegt im Bereich des experimentellen Fehlers, der mit den aktuell besten hochaufgelösten Daten, bei einer Verfeinerung mit dem Hansen \& Coppens-Multipolmodell, erreicht werden kann. 


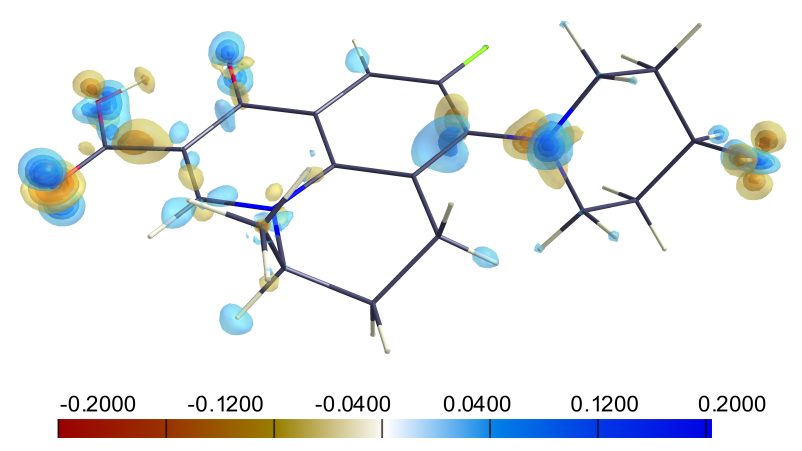

(a) Nadifloxacin

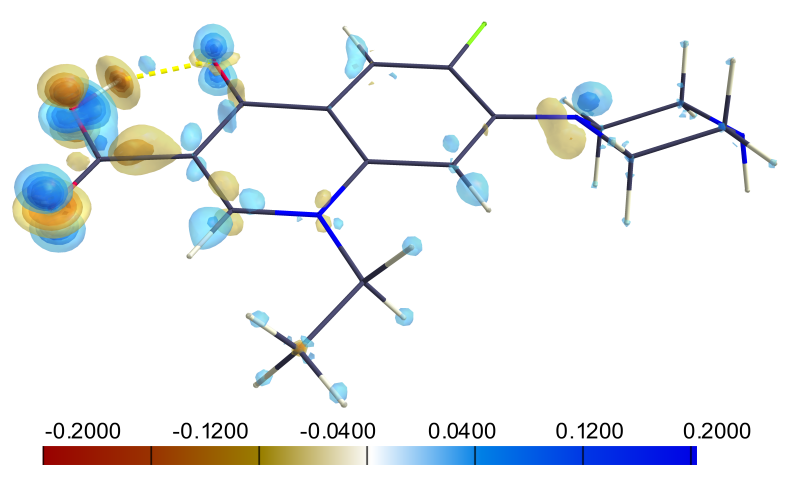

(b) Norflocaxin

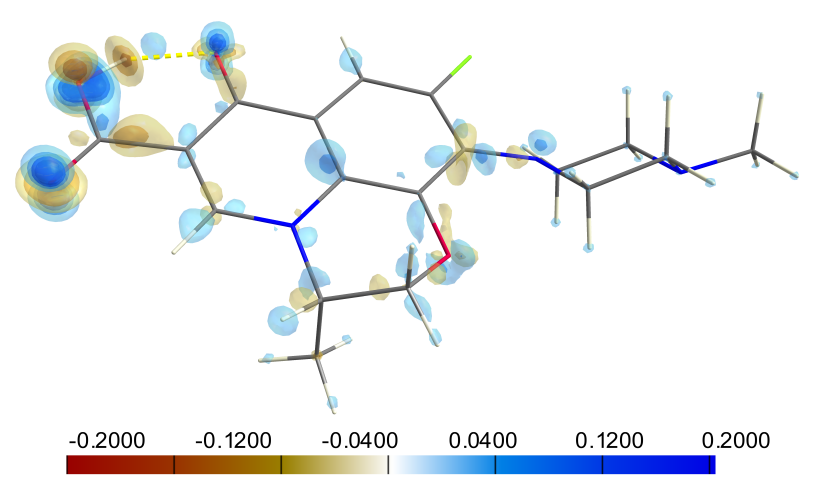

(c) Ofloxacin

Abbildung 8.16: Differenzelektronendichte ungeladener Fluorochinolone mit dem gleichen Protonierungszustand wie in den Strukturen von Nadifloxacin $0,5 \mathrm{H}_{2} \mathrm{O}$ (a), Norflocaxin-Anhydrat (b) und OfloxacinAnhydrat (c). Von der Elektronendichte der Multipolprojektion theoretischer Strukturfaktoren wurde die Elektronendichte des InvariomModells subtrahiert. Die Isooberflächen sind mit MolecoolQT [72] im Bereich von $-0,2 \leq \mathbf{e} / \AA^{3} \leq 0,2$ bei einem Abstand von $0,05 e / \AA^{3}$ eingezeichnet. 


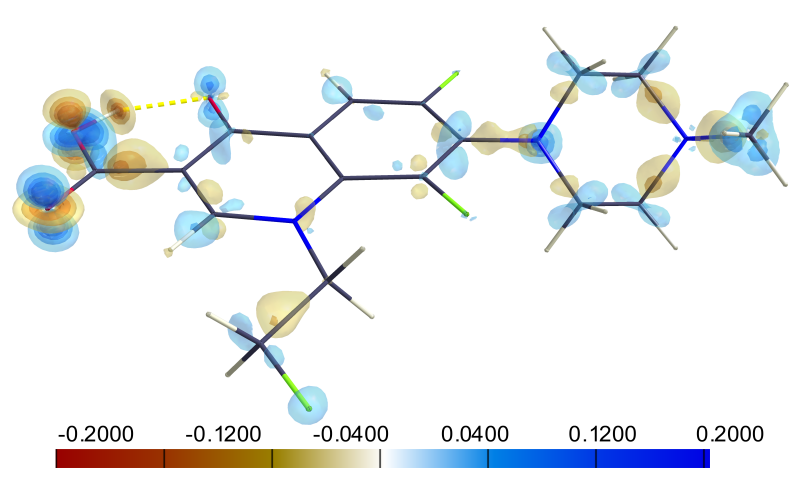

(a) Fleroxacin

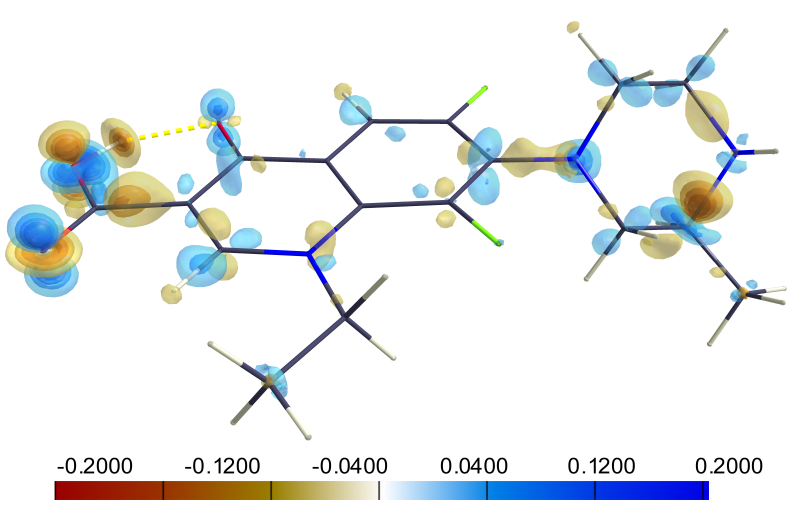

(b) Lomefloxacin

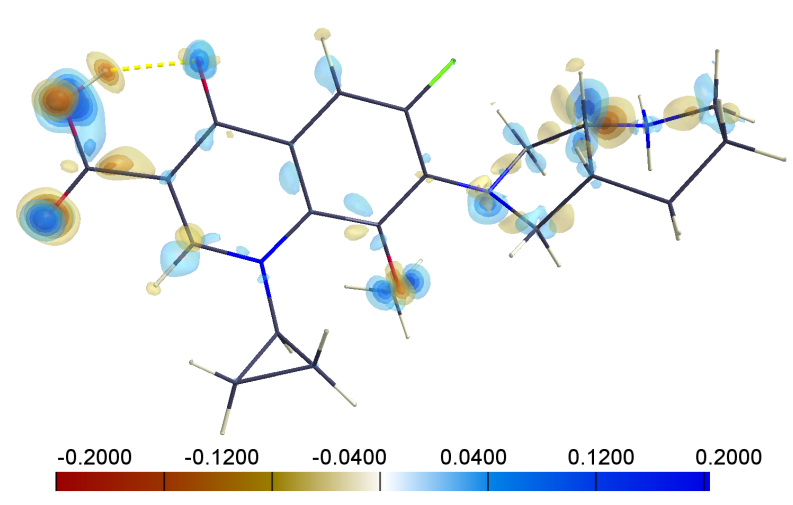

(c) Moxifloxacin

Abbildung 8.17: Differenzelektronendichte kationischer Fluorochinolone mit dem gleichen Protonierungszustand wie in den Strukturen von Fleroxacin $\cdot \mathrm{H}_{2} \mathrm{O} \cdot \mathrm{HCl}$ (a), Lomefloxacin.2 $\mathrm{H}_{2} \mathrm{O} \quad 0,95 \cdot \mathrm{Cl}^{-}$(b) und Moxifloxacin $0,5 \mathrm{H}_{2} \mathrm{O} \cdot 0,5 \mathrm{CH}_{3} \mathrm{OH} \cdot \mathrm{HCl}$ (c). Von der Elektronendichte der Multipolprojektion theoretischer Strukturfaktoren wurde die Elektronendichte des Invariom-Modells subtrahiert. Die Isooberflächen sind mit MolecoolQT [72] im Bereich von $-0,2 \leq \mathbf{e} / \AA^{3} \leq 0,2$ bei einem Abstand von $0,05 e / \AA^{3}$ eingezeichnet. 


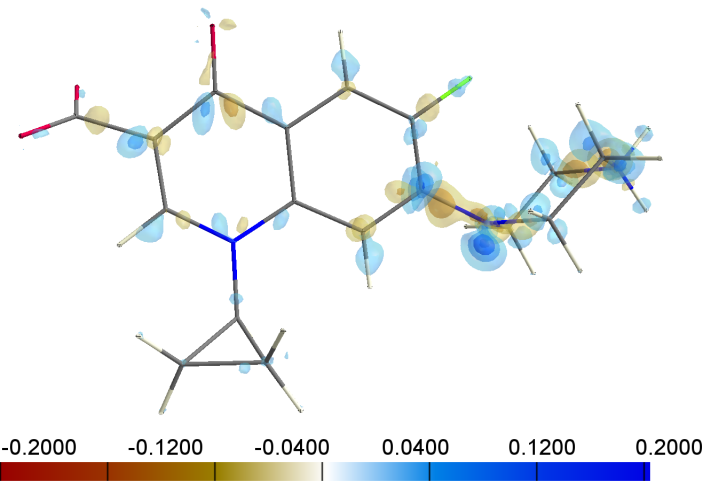

(a) Ciprofloxacin

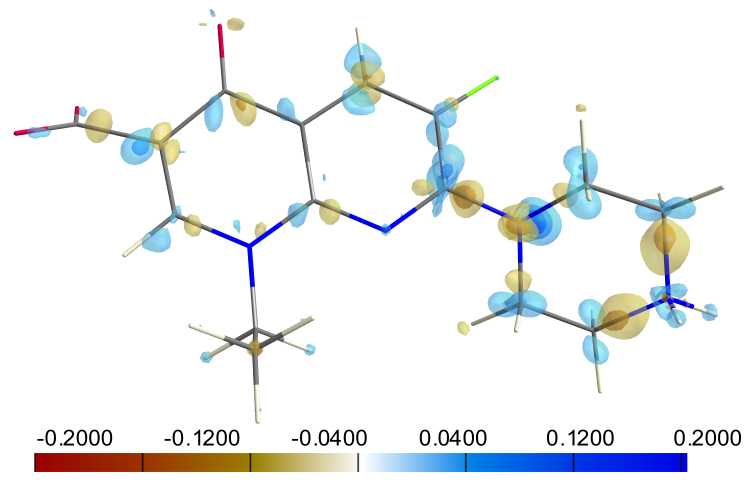

(b) Enoxacin

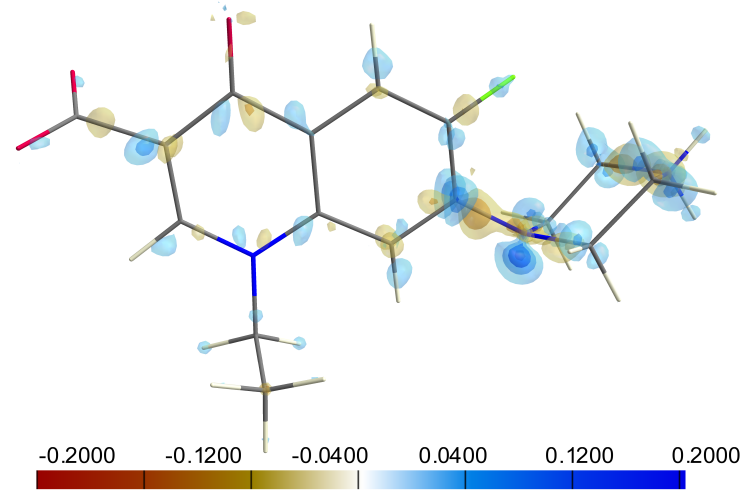

(c) Norflocaxin

Abbildung 8.18: Differenzelektronendichte zwitterionischer Fluorochinolone mit dem gleichen Protonierungszustand wie in den Strukturen von Ciprofloxacin. $6 \quad \mathrm{H}_{2} \mathrm{O}$ (a), Enoxacin $1,7 \quad \mathrm{H}_{2} \mathrm{O}$ und Enoxacin. $3 \mathrm{H}_{2} \mathrm{O}$ (b), Norflocaxin.2 $\mathrm{H}_{2} \mathrm{O}$ (c). Von der Elektronendichte der Multipolprojektion theoretischer Strukturfaktoren wurde die Elektronendichte des Invariom-Modells subtrahiert. DIe Isooberflächen sind mit MOLECOOLQT [72] im Bereich von $-0,2 \leq \mathbf{e} / \AA^{3} \leq 0,2$ bei einem Abstand von 0,05 $e / \AA^{3}$ eingezeichnet. 


\subsection{Zusammenfassung}

Eine Serie höchst-möglich aufgelöster Einkristall-Röntgenbeugungsexperimente wurde mit dem Ziel durchgeführt, genaue Strukturinformationen der antibiotisch wirksamen Fluorochinolone zu erhalten. Die röntgenographische Untersuchung erlaubte die Bestimmung der Protonierungszustände im Festkörper, welche in Abhängigkeit des kokristallisierten Lösungsmittels variiert. Zur Rekonstruktion der molekularen Elektronendichte aus atomaren Fragmenten wurden asphärische Atomformfaktoren für die Invariom-Datenbank theoretisch berechnet und auf die Kristallstrukturen transferiert. Die Modellverbesserung führt zu signifikanten Verbesserungen der kristallographischen Gütefaktoren und erlaubt zugleich die Berechnung molekularer Eigenschaften wie dem elektrostatischen Potential und Dipolmomenten.

Im Gegensatz zu experimentellen Elektronendichtebestimmungen sind für Invariom-Verfeinerung bereits atomar aufgelöste Datensätze ausreichend, wobei die Qualität der Ergebnisse weitestgehend unabhängig von der Güte der Daten ist. Der Ansatz kann, im Gegensatz zu zeitaufwendigen quantenchemischen Rechungen, auch im Hochdurchsatz-Verfahren (engl. high-throughput screening, HTS) eingesetzt werden.

Für zukünftige Untersuchungen ist es prinzipiell auch möglich das InvariomKonzept unabhängig von kristallographischen Studien anzuwenden. Es könnten ebenso die molekulare Geometrie aus Kernspinresonanzspektroskopie (engl. nuclear magnetic resonace, NMR) oder Kraftfeldberechnungen verwendet werden. 


\section{Resümee}

Mit dem Ziel möglichst genaue Strukturinformationen antibiotisch wirksamer Moleküle zu erhalten wurde eine Reihe von hochaufgelöster Einkristall-Röntgenbeugungsexperimente durchgeführt. Aus der Elektronendichteverteilung abgeleitete molekulare Eigenschaften können für die zukünftige Modellierung von Wirkstoff-Rezeptor-Wechselwirkungen verwendet werden.

Beim Makrolid-Antibiotikum Roxithromycin konnte die absolute Struktur mit einer Wellenlänge von 0,5600 Å und Sauerstoff als schwerstem Element über die Bijvoet-Differenzen der subatomar aufgelösten Einkristall-Röntgenbeugungsdaten $\left(\mathrm{d}=0,45 \AA \mathrm{bzw} \cdot \sin \theta / \lambda=1,11 \AA^{-1}\right)$ erfolgen. Durch detaillierte Analyse der verfeinerten Multipolpopulationen wurde eine kontinuierliche Rotationsfehlordnung einiger Wasserstoffatome entdeckt, welche unkorrigiert zu Artefakten des berechneten elektrostatischen Potentials führt. Zusätzlich konnte durch Verfeinerung anharmonischer Auslenkungsparameter der Gram-CharlierExpansion für Atom 027 eine sinnvolle Modellverbesserung erreicht werden. Details der kristallographischen Untersuchung des Roxithromycin-Datensatzes befinden sich auf Seite 83.

Zur Untersuchung molekularer Eigenschaften von neun verschiedenen Fluorochinolon-Antibiotika wurden atomar ( $d=0,82 \AA$ ) bis subatomar aufgelöste Datensätze gemessen oder aus der Literatur bezogen. Wasserstoffatome der Strukturen konnten in allen Fällen beobachtet werden und erlaubten die Bestimmung des Protonierungszustandes der Moleküle im Kristallverband. Restelektronendichtekarten, bei denen die Wasserstoffatome weggelassen wurden (engl. OMIT maps), bestätigen die Positionen aller Wasserstoffatome. Asphärische Atomformfaktoren der Invariom-Datenbank wurden auf zwölf Kristallstrukturen der Fluorochinolone erfolgreich transferiert. Sie wurden anschliessend zur Rekonstruktion der Elektronendichte und Berechnung von Dipolmomenten und elektrostatischen Potentialen verwendet. Es zeigte sich ein gute Übereinstimmung der elektronischen Eigenschaften verschiedener Fluorochi- 
nolone untereinander mit dem selben Protonierungszustand. Dies bestätigt die in der Wirkstoffforschung verbreitete Hypothese, dass Moleküle mit gleicher Wirksamkeit neben einer ähnlichen Konformation auch weitgehend übereinstimmende elektrostatische Eigenschaften aufweisen.

Die Invariom-Datenbank wurde erweitert, um heteroaromatische Verbindungen automatisiert beschreiben zu können. Pseudoatomare Elektronendichtefragmente zur Beschreibung von DNA-Basenpaaren und Fluorochinolon-Antibiotika wurden aus den Wellenfunktionen geometrieoptimierter Modellverbindungen auf das Hansen \& Coppens-Multipolmodell projiziert. Die Annahmen und Näherungen des Invariom-Formalismus wurden sowohl mit experimentellen als auch mit theoretischen Strukturfaktoren validiert. Es zeigte sich für beide Fälle eine gute Übereinstimmung und signifikante Modellverbesserung gegenüber dem Modell unabhängiger Atome (IAM). Die Entwicklung der Invariom-Datenbank erlaubt die Berechnung molekularer Eigenschaften von Röntgenstrukturen. Auch 2D-NMR-Strukturen und niedrig aufgelöste makromolekulare Strukturen können prinzipiell behandelt werden.

In dieser Arbeit wurden molekulare Eigenschaften antibiotisch wirksamer Verbindungen unmittelbar aus der Elektronendichteverteilung abgeleitet. Da nicht alle Datensätze den hohen Anforderungen an die Auflösung für eine experimentelle Elektronendichtebestimmung entsprachen, wurde die Elektronendichte auf Basis des Invariom-Modells verwendet. Die Erweiterung der InvariomDatenbank erlaubt nun die Beschreibung beliebiger heteroaromatischer organischer Moleküle und kann zukünftig vermehrt zur Bestimmung molekularer Eigenschaften pharmakologisch aktiver Substanzen verwendet werden. 


\section{Summary}

A series of high resolution X-ray experiments of antibiotics was carried out to obtain structural information as precise as possible. Molecular properties derived from the electron density distribution can be used to model drug receptor interactions in future.

Single-crystal X-ray data of macrolide antibiotic roxithromycin with subatomic resolution $\left(\mathrm{d}=0.45 \AA\right.$ or $\left.\sin \theta / \lambda=1.11 \AA^{-1}\right)$ allowed absolute structure determination using Bijvoet differences, even though the data were measured at a wavelength of $0.5600 \AA$ with oxygen as the heaviest element present. Detailed analysis and validation of the multipole refinement revealed continuous rotational disorder of some hydrogen atoms, which results in an artifact of the electrostatic potential, if not modelled appropriately. Furthermore, displacement parameters of atom $\mathrm{O} 27$ were refined using Gram-Charlier expansion of third order to describe the anharmonicity of the atomic probability distribution. Crystallographic details are summarised on page 83.

To study molecular properties of nine different fluoroquinolone antibiotics, datasets were collected and reused from literature. Datasets with atomic $(\mathrm{d}=$ $0.82 \AA$ A) or subatomic resolutions were examined. In these structures, hydrogen atoms could be observed, which allowed deriving the protonation state of the molecules in the crystal environment. OMIT maps for hydrogen atoms showed that all hydrogen positions were correct. Aspherical scattering factors of the invariom database were successfully transfered to twelve crystal structures of fluoroquinolones. Invarioms were successfully used to reconstruct the electron density and to calculate dipole moments and electrostatic potentials directly from the crystal structures. Similarities in the molecular electrostatic properties of the different fluoroquinolone molecules with the same protonation state were revealed in this way. This finding confirms the prevalent assumption that molecules with same pharmacological properties have not only a similar conformation, but also similar electrostatic properties. 
The invariom database was extended to automatically deal with heteroaromatic compounds. Pseudoatomic density fragments suitable to reconstruct the electron density of DNA-base pairs and fluoroquinolone antibiotics were projected from molecular wavefunctions of geometry-optimized model compounds onto pseudoatoms within the electron density representation of the Hansen \& Coppens multipole model. The invariom database was validated with both experimental and theoretical approaches giving a good agreement and significant model improvements compared to the independent atom model (IAM). Improvements are due to taking into account the aspherical electron density. Recent developments of the invariom database enable molecular property calculations for X-ray structures. 2D-NMR structures and low-resolution macromolecular $\mathrm{X}$-ray structures can in principle also be dealt with.

In this work molecular properties of a number of antibiotics were calculates directly from the electron density distribution. Since the resolution was not suitable for an experimental charge-density determination in some cases, the invariom model was used instead of a free multipole refinement. The extended invariom database now covers all organic heteroaromatic molecules and can be used more often to calculate molecular properties of pharmacological active substances in the future. 


\section{Literaturverzeichnis}

[1] D. E. Koshland. Angewandte Chemie International Edition 33 (1995), 2375-2378

[2] S. Riethmiller. Chemotherapy 51 (2005), 234-242

[3] N. Lloyd, H. Morgan, B. Nicholson, R. Ronimus. Angewandte Chemie International Edition 44 (2005), 941-944

[4] G. Taubes. Science 321 (2008), 356-361

[5] G. Klebe. Wirkstoffdesign: Entwurf und Wirkung von Arzneistoffen. Spektrum Akademischer Verlag, Heidelberg (2009)

[6] P. Luger. Modern X-Ray Analysis on Single Crystals. W. de Gruyter, Heidelberg and Berlin (1980)

[7] W. Massa. Kristallstrukturbestimmung. Teubner Studienbücher. B. G. Teubner, Stuttgart, 2. Auflage (1996)

[8] W. F. Kuhs. Acta Cryst. A 48 (1992), 80-98

[9] K. N. Trueblood, H.-B. Bürgi, H. Burzlaff, J. D. Dunitz, C. M. Gramaccioli, H. H. Schulz, U. Shmueli, S. C. Abrahams. Acta Cryst. A 52 (1996), 770-781

[10] F. L. Hirshfeld. Acta Cryst. A 32 (1976), 239-244

[11] R. E. Rosenfield, K. Trueblood, J. D. Dunitz. Acta Cryst. A 34 (1978), 828-829

[12] C. F. Gauss. Abhandlungen zur Methode der kleinsten Quadrate. Anton Börsch und Paul Simon, Berlin (1887)

[13] H. D. Flack. Acta Cryst. A 39 (1983), 876-881 
[14] J. M. Bijvoet, A. F. Peerdeman, A. J. van Bommel. Nature 168 (1951), 271-272

[15] S. Parsons, H. Flack. In Acta Cryst., Band 60 (2004)

[16] A. L. Thompson, D. J. Wadkin. J. Appl. Cryst. 44 (2011), 1017-1022

[17] B. Rupp. Biomolecular Crystallography: Principles, Practice and Application to Structural Biology. Garland Science, New York (2009)

[18] L. Palatinus, S. van Smaalen. Acta Cryst. A 58 (2002), 559-567

[19] J. Netzel, A. Hofmann, S. van Smaalen. Accurate charge density of $\alpha$ glycine by the maximum entropy method (2008)

[20] J. Netzel, S. van Smaalen. Acta Cryst. B 65 (2009), 624-38

[21] P. A. Tipler, G. Mosca. Physik für Wissenschaftler und Ingenieure. Elsevier - Spektrum akademischer Verlag, München, 2. Auflage (2004)

[22] G. Wedler. Lehrbuch der Physiklischen Chemie. Wiley-VCH, Weinheim, 4. Auflage (1997)

[23] R. McWeeny. Acta Cryst. A 4 (1951), 513-519

[24] C. Giacovazzo, H. L. Monaco, D. Viterbo, F. Scordari, G. Gilli, G. Zanotti, M. Catti. Fundamentals of Crystallography. Nummer 2 in IUCr Texts on Crystallography. Oxford University Press, Oxford, 1. Auflage (1992)

[25] Z. Su, P. Coppens. J. Appl. Cryst. 23 (1990), 71-73

[26] P. Coppens. X-Ray Charge Densities and Chemical Bonding. Nummer 4 in IUCr Texts on Crystallography. Oxford University Press, Oxford, 1. Auflage (1997)

[27] U. Shmueli, (Hg.) International Tables for X-ray Crystallography Volume B: Reciprocal Space, Band B. Kluwer Academic Publishers, Dordrecht, 2. Auflage (2001)

[28] R. F. Stewart, E. R. Davidson, W. T. Simpson. J. Chem. Phys. 42 (1965), 3175-3187

[29] E. Clementi, C. Roetti. At. Data Nucl. Data Tables 14 (1974), 177-478

[30] E. Prince, (Hg.) International Tables for X-ray Crystallography Volume C: Mathematical, Physical and Chemical Tables, Band C. Kluwer Academic Publishers, Dordrecht, 3. Auflage (2004) 
[31] N. K. Hansen, P. Coppens. Acta Cryst. A 34 (1978), 909-921

[32] C. Jelsch, V. Pichon-Pesme, C. Lecomte, A. Aubry. Acta Cryst. D 54 (1998), 1306-1318

[33] B. Dittrich, C. B. Hübschle, P. Luger, M. A. Spackman. Acta Cryst. D 62 (2006), 1325-1335

[34] P. M. Dominiak, A. Volkov, X. Li, M. Messerschmidt, P. Coppens. J. Chem. Theory Comput. 2 (2007), 232-247

[35] V. R. Hathwar, T. S. Thakur, T. N. G. Row, G. R. Desiraju. Crystal Growth \& Design 11 (2011), 616-623

[36] J. M. Bąk, S. Domagala, C. Hübschle, C. Jelsch, B. Dittrich, P. M. Dominiak. Acta Cryst. A 67 (2011), 141-153

[37] D. Jayatilaka, B. Dittrich. Acta Cryst. A 64 (2008), 383-393

[38] D. Jayatilaka, D. J. Grimwood. Acta Cryst. A 57 (2001), 76-86

[39] D. J. Grimwood, D. Jayatilaka. Acta Cryst. A 57 (2001), 87-100

[40] D. Jayatilaka, P. Munshi, M. J. Turner, J. A. K. Howard, M. A. Spackman. Phys. Chem. Chem. Phys. 11 (2009), 7209-7218

[41] F. L. Hirshfeld. Theoret. Chim. Acta (Berl.) 44 (1977), 129-138

[42] F. H. Allen, I. J. Bruno. Acta Cryst. B 66 (2010), 380-386

[43] G. M. Sheldrick. Acta Cryst. A 64 (2008), 112-122

[44] B. Guillot, L. Viry, R. Guillot, C. Lecomte, C. Jelsch. J. Appl. Cryst. 34 (2001), 214-223

[45] C. Jelsch, B. Guillot, A. Lagoutte, C. Lecomte. J. Appl. Cryst. 38 (2005), 38-54

[46] A. Volkov, P. Macchi, L. J. Farrugia, C. Gatti, P. Mallinson, T. Richter, T. Koritsánszky. XD2006 - A Computer Program Package for Multipole Refinement, Topological Analysis of Charge Densities and Evaluation of Intermolecular Energies from Experimental or Theoretical Structure Factors. (2006)

[47] R. F. Stewart. J. Chem. Phys. 53 (1970), 205-213 
[48] R. F. Stewart, M. A. Spackman, C. Flensburg. VAlRAY98, Users Manual. Carnegie Mellon University and University of Copenhagen, Pittsburgh, USA and Denmark (1998)

[49] C. B. Hübschle, P. Luger, B. Dittrich. J. Appl. Cryst. 40 (2007), 623-627

[50] A. Volkov, M. Messerschmidt, P. Coppens. Acta Cryst. D 63 (2007), 160170

[51] P. M. Dominiak, A. Volkov, A. P. Dominiak, K. N. Jarzembska, P. Coppens. Acta Cryst. D (2009), 485-499

[52] D. Jayatilaka, D. J. Grimwood. Computational Science - ICCS 20032660 (2003), 142-151

[53] R. F. W. Bader, P. Lode, A. Popelier, T. A. Keith. Angew. Chem. 106 (1994), 647-659

[54] R. F. W. Bader. Atoms in Molecules: A Quantum Theory. Nummer 22 in The International Series of Monographs on Chemistry. Clarendon Press, Oxford, 2. Auflage (1994)

[55] T. S. Koritsánszky, P. Coppens. Chem. Rev. 101 (2001), 1583-1628

[56] M. A. Spackman, P. G. Byrom. Chem. Phys. Lett. 267 (1997), 215-220

[57] Z. Su, P. Coppens. Acta Cryst. A 48 (1992), 188-197

[58] A. Volkov, H. F. King, P. Coppens, L. J. Farrugia. Acta Cryst. A 62 (2006), $400-408$

[59] A. D. Buckingham. Permanent and Induced Molecular Moments and LongRange Intermolecular Forces, Band 12. John Wiley \& Sons, Inc (1967)

[60] J. J. McKinnon, M. A. Spackman, A. S. Mitchell. Acta Cryst. B 60 (2004), $627-668$

[61] M. A. Spackman, J. J. McKinnon, D. Jayatilaka. Cryst. Eng. Comm. 10 (2008), 377-388

[62] M. A. Spackman, D. Jayatilaka. Cryst. Eng. Comm. 11 (2009), 19-32

[63] P. Politzer, J. S. Murray, Z. Preralta-Inga. Int. J. Quant. Chem. 85 (2001), 676-684

[64] C. B. Hübschle, P. Luger. J. Appl. Cryst. 39 (2006), 901-904 
[65] C. B. Hübschle, B. Dittrich, S. Grabowsky, M. Messerschmidt, P. Luger. Acta Cryst. B 64 (2008), 363-374

[66] J. J. Holstein, P. Luger, R. Kalinowski, S. Mebs, C. Paulmann, B. Dittrich. Acta Cryst. B 66 (2010), 568-577

[67] Y. A. Abramov. Acta Cryst. A 53 (1997), 264-272

[68] E. Espinosa, E. Molins, C. Lecomte. Chem. Phys. Lett. 285 (1998), 170173

[69] B. Dittrich, T. Koritsánszky, P. Luger. Angew. Chem. 43 (2004), 27732776

[70] B. Dittrich, C. B. Hübschle, M. Messerschmidt, R. Kalinowski, D. Girnt, P. Luger. Acta Cryst. A 61 (2005), 314-320

[71] A. L. Allred, E. G. Rochow. J. Inorg. Nucl. Chem. 5 (1958), 264-268

[72] C. B. Hübschle, B. Dittrich. J. Appl. Cryst. 44 (2011), 238-240

[73] M. J. Frisch, G. W. Trucks, H. B. Schlegel, M. A. R. G. E. Scuseria, J. R. Cheeseman, J. A. M. Jr., K. N. K. T. Vreven, J. C. Burant, J. M. Millam, S. S. Iyengar, V. B. J. Tomasi, B. Mennucci, M. Cossi, G. Scalmani, G. A. P. N. Rega, H. Nakatsuji, M. Hada, M. Ehara, R. F. K. Toyota, J. Hasegawa, M. Ishida, T. Nakajima, Y. Honda, H. N. O. Kitao, M. Klene, X. Li, J. E. Knox, H. P. Hratchian, V. B. J. B. Cross, C. Adamo, J. Jaramillo, R. Gomperts, O. Y. R. E. Stratmann, A. J. Austin, R. Cammi, C. Pomelli, P. Y. A. J. W. Ochterski, K. Morokuma, G. A. Voth, P. Salvador, V. G. Z. J. J. Dannenberg, S. Dapprich, A. D. Daniels, O. F. M. C. Strain, D. K. Malick, A. D. Rabuck, J. B. F. K. Raghavachari, J. V. Ortiz, Q. Cui, A. G. Baboul, J. C. S. Clifford, B. B. Stefanov, G. Liu, A. Liashenko, I. K. P. Piskorz, R. L. Martin, D. J. Fox, T. Keith, C. Y. P. M. A. Al-Laham, A. Nanayakkara, M. Challacombe, B. J. P. M. W. Gill, W. Chen, M. W. Wong, C. Gonzalez, J. A. Pople. Gaussian 03, Revision D.01. Technischer Bericht, Gaussian, Inc., Wallingford CT (2004)

[74] S. Ali, M. Banck, R. Braithwaite, J. Bunt, D. Curtis, N. Fox, M. Hanwell, G. Hutchison, B. Jacob, D. Lonie, J. Mantha, T. Margraf, C. Niehaus, S. Ochsenreither, K. Tokarev, T. Vandermeersch. Avogadro: an open-source molecular builder and visualization tool. Version 0.9.4. http://avogadro.openmolecules.net/. Technischer Bericht, none (2010)

[75] D. Weininger. J. Chem. Inform. Comput. Sci. 28 (1988), 31-36 
[76] A. K. Rappé, C. J. Casewit, K. S. Colwell, W. A. Goddard, W. M. Skid, E. R. Bernstein. J. Am. Chem. Soc. 114 (1992), 10024-10039

[77] W. Kohn, A. D. Becke, R. G. Parr. J. Phys. Chem. 100 (1996), 1297412980

[78] R. G. Parr, W. Yang. Density Functional Theory in Atoms and Molecules. Oxford University Press, New York, 2. Auflage (1989)

[79] T. H. Dunning. J. Chem. Phys. 53 (1970), 2823-2833

[80] T. Koritsánszky, A. Volkov, P. Coppens. Acta Cryst. A 58 (2002), 464-472

[81] S. Grabowsky, R. Kalinowski, M. Weber, D. Förster, C. Paulmann, P. Luger. Acta Cryst. B 65 (2009), 488-501

[82] B. Dittrich, P. Munshi, M. A. Spackman. Acta Cryst. B 63 (2007), 505509

[83] B. Dittrich, J. E. Warren, F. P. A. Fabbiani, W. Morgenroth, B. Corry. Phys. Chem. Chem. Phys. 11 (2009), 2601-2609

[84] R. Guha, M. T. Howard, G. R. Hutchison, P. Murray-Rust, H. Rzepa, C. Steinbeck, J. K. Wegner, E. L. Willighagen. Journal of Chemical Information and Modeling 46 (2006), 991-998

[85] M. Banck, G. Hutchison, C. Morley, N. O’Boyle, T. Vandermeersch. The Open Babel Package, Version 2.3.0. Technischer Bericht, http://openbabel.org. Babel

[86] F. Schlunzen, R. Zarivach, J. Harms, A. Bashan, A. Tocilj, R. Albrecht, A. Yonath, F. Franceschi. Nature 321 (2001), 814-821

[87] W. Kabsch. J. Appl. Cryst. 26 (1993), 795-800

[88] W. Hofheinz, H. Griesbach, H. Friebolin. Tetrahedron 18 (1962), 12651274

[89] C. B. Hübschle, G. M. Sheldrick, B. Dittrich. J. Appl. Cryst. 44 (2011), $1281-1284$

[90] T. Koritsánszky, T. Richter, P. Macchi, A. Volkov, C. Gatti, S. Howard, P. R. Mallinson, L. Farrugia, Z. W. Su, N. K. Hansen. XD - a Computer Program Package for Multipole Refinement and Topological Analysis of Electron Densities from Diffraction Data.. Technischer Bericht, Freie Universität Berlin, Berlin (2003) 
[91] A. Ø. Madsen, H. O. Sørensen, C. Flensburg, R. F. Stewart, S. Larsen. Acta Cryst. A 60 (2004), 550-561

[92] A. Ø. Madsen. J. Appl. Cryst. 39 (2006), 757-758

[93] V. Schomaker, K. N. Trueblood. Acta Cryst. B 24 (1968), 63-76

[94] R. F. W. Bader. Atoms in Molecules: A Guantum Theory. Nummer 22 in The International Series of Monographs on Chemistry. Clarendon Press, Oxford, 1. Auflage (1990)

[95] P. Müller, R. Herbst-Irmer, A. Spek, T. Schneider, M. Sawaya. Crystal Structure Refinement: A Crystallographer's Guide to SHELXL. Oxford University Press, New York, 1. Auflage (2006)

[96] J. M. Bąk, P. M. Dominiak, C. C. Wilson, K. Wozniak. Acta Cryst. A 65 (2009), 490-500

[97] K. Meindl, R. Herbst-Irmer, J. Henn. Acta Cryst. A 66 (2010), 362-371

[98] M. A. Spackman, P. Munshi, B. Dittrich. ChemPhysChem 8 (2007), 2051-2063

[99] L. A. Mitscher. Chem. Rev. 105 (2005), 559-592

[100] B. Wiedemann, P. Heisig. Chemotherapie Journal 8 (1999), 99-107

[101] B. D. Bax, P. F. Chan, D. S. Eggleston, A. Fosberry, D. R. Gentry, F. Gorrec, I. Giordano, M. M. Hann, A. Hennessy, M. Hibbs, J. Huang, E. Jones, J. Jones, K. K. Brown, C. J. Lewis, E. W. May, M. R. Saunders, O. Singh, C. E. Spitzfaden, C. Shen, A. Shillings, A. J. Theobald, A. Wohlkonig, N. D. Pearson, M. N. Gwynn. Nature 466 (2010), 935-943

[102] M. Parvez, S. Arayne, N. Sultanab, A. Z. Siddiqic. Acta Cryst. C 60 (2004), o281-0283

[103] X. Zheng, H. Zhao, L. Jin. Acta Cryst. C 58 (2002), o596-o597

[104] K. Ravikumar, B. Sridhar. Acta Cryst. C 62 (2006), o478-o482

[105] A. Sivalakshmidevi, K. Vyas, G. O. Reddy. Acta Cryst. C 56 (2000), e1 15e116

[106] I. Turel, P. Bukovec, M. Quirós. Int. J. Pharm. 152 (1997), 59-65

[107] I. Turel, A. Golobic. Anal. Sci. 19 (2003), 329-330 
[108] I. Yoon, K. Bark, M. S. Yang, S. S. Lee. Anal. Sci. 19 (2003), x11-x12

[109] M. Kido, K. Hashimoto. Chem. Pharm. Bull. 42 (1994), 872-876

[110] S. Basavoju, D. Bostrom, S. P. Velaga. Cryst. Growth Des. 12 (2006), 2699-2708

[111] A. J. Florence, A. R. Kennedy, N. Shankland, E. Wright, A. Al-Rubayi. Acta Cryst. C 56 (2000), 1372-1372

[112] S. Mahapatra, K. N. Venugopala, T. N. G. Row. Cryst. Growth Des. 10 (2010), 1866-1870

[113] L. J. Farrugia. J. Appl. Cryst. 32 (1999), 837-838

[114] Bruker AXS Inc., Madison, WI. SAINT data processing software, version 7.61A (1995-2009)

[115] G. M. Sheldrick. SADABS version 2008/2. A Program for area detector absorption and other corrections. Technischer Bericht, University of Göttingen, Germany (2008)

[116] B. Dittrich, C. B. Hübschle, J. J. Holstein, F. P. A. Fabbiani. J. Appl. Cryst. 42 (2009), 1110-1121

[117] Y. A. Abramov, A. V. Volkov, P. Coppens. Chem. Phys. Lett. 311 (1999), $81-86$

[118] A. Volkov, P. Coppens. Acta Cryst. A 57 (2001), 395-405

[119] B. Dittrich, M. A. Spackman. Acta Cryst. A 63 (2007), 426-436

[120] M. A. Spackman, P. G. Byrom. Acta Cryst. B 53 (1997), 553-564

[121] A. Volkov, Y. Abramov, P. Coppens, C. Gatti. Acta Cryst. A 56 (2000), 332-339 



\section{Anhang A}

\section{Tabellen}

\section{A.1 Roxithromycin}

\section{A.1.1 Locale Koordinatensysteme, Hilfsatome und chemical con- traints der gemischten Multipolverfeinerung (GMV).}

\begin{tabular}{|c|c|c|c|c|c|c|c|c|c|c|c|c|}
\hline ГOM & 11 & $\mathrm{AX} 1$ & & & $n 2$ & & & & & & & \\
\hline $\mathrm{O}(14)$ & DUM1 & $Z$ & $\mathrm{O}(14)$ & $\mathrm{C}(1)$ & $\mathrm{Y}$ & $\mathrm{R}$ & 2 & 1 & 1 & 4 & MM2 & \\
\hline $\mathrm{O}(21)$ & DUM2 & $\mathrm{X}$ & $\mathrm{O}(21)$ & $\mathrm{C}(1)$ & Z & $\mathrm{R}$ & 2 & 1 & 2 & 4 & MM2 & \\
\hline $\mathrm{O}(22)$ & $\mathrm{C}(6)$ & $\mathrm{X}$ & $\mathrm{O}(22)$ & $\mathrm{H}(22)$ & $\mathrm{Y}$ & $\mathrm{R}$ & 2 & 1 & 3 & 4 & $\mathrm{M}$ & \\
\hline $\mathrm{O}(23)$ & $\mathrm{C}(11)$ & $\mathrm{X}$ & $\mathrm{O}(23)$ & $\mathrm{H}(23)$ & $\mathrm{Y}$ & $\mathrm{R}$ & 2 & 1 & 3 & 4 & M & \\
\hline $\mathrm{O}(24)$ & $\mathrm{C}(12)$ & $\mathrm{X}$ & $\mathrm{O}(24)$ & $\mathrm{H}(24)$ & $\mathrm{Y}$ & $\mathrm{R}$ & 2 & 1 & 3 & 4 & M & \\
\hline $\mathrm{O}(25)$ & $\mathrm{C}(68)$ & $\mathrm{X}$ & $\mathrm{O}(25)$ & $\mathrm{N}(1)$ & $\mathrm{Y}$ & $\mathrm{R}$ & 2 & 1 & 4 & 4 & M & \\
\hline $\mathrm{O}(26)$ & DUM3 & $Z$ & $\mathrm{O}(26)$ & $\mathrm{C}(68)$ & $\mathrm{Y}$ & $\mathrm{R}$ & 2 & 1 & 1 & 4 & MM2 & \\
\hline $\mathrm{O}(27)$ & DUM4 & $Z$ & $\mathrm{O}(27)$ & $\mathrm{C}(70)$ & $\mathrm{Y}$ & $\mathrm{R}$ & 2 & 1 & 1 & 4 & MM2 & \\
\hline $\mathrm{O}(5)$ & DUM5 & $Z$ & $\mathrm{O}(5)$ & $\mathrm{C}(5)$ & $\mathrm{Y}$ & $\mathrm{R}$ & 2 & 1 & 1 & 4 & MM2 & $\mathrm{O}(14)$ \\
\hline $\mathrm{O}(58)$ & DUM6 & $Z$ & $\mathrm{O}(58)$ & $C(50)$ & $\mathrm{Y}$ & $\mathrm{R}$ & 2 & 1 & 1 & 4 & MM2 & $\mathrm{O}(14)$ \\
\hline $\mathrm{O}(59)$ & $C(51)$ & $\mathrm{X}$ & $\mathrm{O}(59)$ & H(59) & $\mathrm{Y}$ & $\mathrm{R}$ & 2 & 1 & 3 & 4 & M & \\
\hline $\mathrm{O}(3)$ & DUM7 & $Z$ & $\mathrm{O}(3)$ & $\mathrm{C}(3)$ & $\mathrm{Y}$ & $\mathrm{R}$ & 2 & 1 & 1 & 4 & MM2 & $\mathrm{O}(14)$ \\
\hline $\mathrm{O}(38)$ & DUM8 & $Z$ & $\mathrm{O}(38)$ & $\mathrm{C}(30)$ & $\mathrm{Y}$ & $\mathrm{R}$ & 2 & 1 & 1 & 4 & MM2 & $\mathrm{O}(14)$ \\
\hline $\mathrm{O}(39)$ & $\mathrm{C}(33)$ & $\mathrm{X}$ & $\mathrm{O}(39)$ & $\mathrm{H}(39)$ & $\mathrm{Y}$ & $\mathrm{R}$ & 2 & 1 & 3 & 4 & M & \\
\hline $\mathrm{O}(40)$ & DUM9 & $Z$ & $\mathrm{O}(40)$ & $\mathrm{C}(32)$ & $\mathrm{Y}$ & $\mathrm{R}$ & 2 & 1 & 1 & 4 & MM2 & $\mathrm{O}(14)$ \\
\hline $\mathrm{O}(80)$ & DUM10 & $Z$ & $\mathrm{O}(80)$ & $\mathrm{H}(80 \mathrm{~A})$ & $\mathrm{Y}$ & $\mathrm{R}$ & 2 & 1 & 5 & 4 & MM2 & \\
\hline $\mathrm{N}(2)$ & DUM11 & $Z$ & $\mathrm{~N}(2)$ & $\mathrm{C}(52)$ & $\mathrm{Y}$ & $\mathrm{R}$ & 2 & 2 & 6 & 4 & 3 & \\
\hline $\mathrm{N}(1)$ & $\mathrm{C}(9)$ & $\mathrm{X}$ & $\mathrm{N}(1)$ & $\mathrm{O}(25)$ & $\mathrm{Y}$ & $\mathrm{R}$ & 2 & 2 & 7 & 4 & M & \\
\hline$C(1)$ & $\mathrm{O}(21)$ & $\mathrm{X}$ & $C(1)$ & $\mathrm{O}(14)$ & $\mathrm{Y}$ & $\mathrm{R}$ & 2 & 3 & 8 & 4 & M & \\
\hline $\mathrm{C}(2)$ & $\mathrm{H}(2)$ & Z & $\mathrm{C}(2)$ & $\mathrm{C}(1)$ & $\mathrm{X}$ & $\mathrm{R}$ & 2 & 3 & 9 & 4 & $3 \mathrm{M}$ & \\
\hline $\mathrm{C}(3)$ & $\mathrm{O}(3)$ & $\mathrm{X}$ & $\mathrm{C}(3)$ & $\mathrm{C}(2)$ & $\mathrm{Y}$ & $\mathrm{R}$ & 2 & 3 & 10 & 4 & M & \\
\hline $\mathrm{C}(4)$ & $\mathrm{H}(4)$ & Z & $\mathrm{C}(4)$ & $\mathrm{C}(3)$ & $\mathrm{X}$ & $\mathrm{R}$ & 2 & 3 & 9 & 4 & $3 \mathrm{M}$ & $\mathrm{C}(2)$ \\
\hline $\mathrm{C}(5)$ & $\mathrm{O}(5)$ & $\mathrm{X}$ & $\mathrm{C}(5)$ & $\mathrm{C}(4)$ & $\mathrm{Y}$ & $\mathrm{R}$ & 2 & 3 & 10 & 4 & M & $\mathrm{C}(3)$ \\
\hline $\mathrm{C}(6)$ & $\mathrm{O}(22)$ & Z & $\mathrm{C}(6)$ & $\mathrm{C}(5)$ & $\mathrm{X}$ & $\mathrm{R}$ & 2 & 3 & 11 & 4 & $3 \mathrm{M}$ & \\
\hline $\mathrm{C}(7)$ & DUM12 & Z & $\mathrm{C}(7)$ & $\mathrm{C}(6)$ & Y & $\mathrm{R}$ & 2 & 3 & 12 & 4 & MM2 & \\
\hline $\mathrm{C}(8)$ & $\mathrm{H}(8)$ & $Z$ & $\mathrm{C}(8)$ & $\mathrm{C}(7)$ & $\mathrm{X}$ & $\mathrm{R}$ & 2 & 3 & 9 & 4 & $3 \mathrm{M}$ & $\mathrm{C}(2)$ \\
\hline $\mathrm{C}(9)$ & $\mathrm{N}(1)$ & $Z$ & $\mathrm{C}(9)$ & $\mathrm{C}(8)$ & $\mathrm{Y}$ & $\mathrm{R}$ & 2 & 3 & 13 & 4 & 2 & \\
\hline
\end{tabular}




\begin{tabular}{|c|c|c|c|c|c|c|c|c|c|c|c|c|}
\hline ATOM & ATOMO & AX1 & ATOM1 & ATOM2 & AX2 & $\mathrm{R} / \mathrm{L}$ & TP & TBL & KAP & LMX & SITESYM & I CHEMCON \\
\hline $\mathrm{C}(10)$ & $\mathrm{H}(10)$ & $Z$ & $\mathrm{C}(10)$ & $\mathrm{C}(9)$ & $\mathrm{X}$ & $\mathrm{R}$ & 2 & 3 & 9 & 4 & $3 \mathrm{M}$ & $\mathrm{C}(2)$ \\
\hline $\mathrm{C}(11)$ & $\mathrm{O}(23)$ & $\mathrm{X}$ & $\mathrm{C}(11)$ & $C(10)$ & Y & $\mathrm{R}$ & 2 & 3 & 10 & 4 & M & $\mathrm{C}(3)$ \\
\hline $\mathrm{C}(12)$ & $\mathrm{O}(24)$ & $Z$ & $\mathrm{C}(12)$ & $\mathrm{C}(11)$ & $\mathrm{X}$ & $\mathrm{R}$ & 2 & 3 & 11 & 4 & $3 \mathrm{M}$ & $\mathrm{C}(6)$ \\
\hline $\mathrm{C}(13)$ & $\mathrm{O}(14)$ & $\mathrm{X}$ & $\mathrm{C}(13)$ & $\mathrm{C}(12)$ & $\mathrm{Y}$ & $\mathrm{R}$ & 2 & 3 & 10 & 4 & M & $\mathrm{C}(3)$ \\
\hline$C(50)$ & $\mathrm{C}(51)$ & $\mathrm{X}$ & $\mathrm{C}(50)$ & $\mathrm{H}(50)$ & Y & $\mathrm{R}$ & 2 & 3 & 14 & 4 & M & \\
\hline $\mathrm{C}(51)$ & $\mathrm{O}(59)$ & $\mathrm{X}$ & $\mathrm{C}(51)$ & $C(50)$ & $\mathrm{Y}$ & $\mathrm{R}$ & 2 & 3 & 10 & 4 & M & $\mathrm{C}(3)$ \\
\hline $\mathrm{C}(52)$ & $\mathrm{H}(52)$ & $\mathrm{X}$ & $C(52)$ & $\mathrm{N}(2)$ & $\mathrm{Y}$ & $\mathrm{R}$ & 2 & 3 & 15 & 4 & M & \\
\hline C(53) & DUM13 & $Z$ & $\mathrm{C}(53)$ & $\mathrm{C}(52)$ & Y & $\mathrm{R}$ & 2 & 3 & 12 & 4 & MM2 & $\mathrm{C}(7)$ \\
\hline $\mathrm{C}(54)$ & $\mathrm{O}(58)$ & $\mathrm{X}$ & $\mathrm{C}(54)$ & $\mathrm{C}(53)$ & $\mathrm{Y}$ & $\mathrm{R}$ & 2 & 3 & 10 & 4 & M & $\mathrm{C}(3)$ \\
\hline $\mathrm{C}(55)$ & $\mathrm{C}(54)$ & Z & $C(55)$ & $\mathrm{H}(55 \mathrm{~A})$ & $\mathrm{Y}$ & $\mathrm{R}$ & 2 & 3 & 16 & 4 & 3 & \\
\hline $\mathrm{C}(56)$ & $\mathrm{N}(2)$ & $Z$ & $\mathrm{C}(56)$ & $\mathrm{H}(56 \mathrm{~A})$ & $\mathrm{Y}$ & $\mathrm{R}$ & 2 & 3 & 17 & 4 & 3 & \\
\hline $\mathrm{C}(57)$ & $\mathrm{N}(2)$ & $Z$ & $\mathrm{C}(57)$ & $\mathrm{H}(57 \mathrm{~A})$ & $\mathrm{Y}$ & $\mathrm{R}$ & 2 & 3 & 17 & 4 & 3 & C(56) \\
\hline $\mathrm{C}(30)$ & $\mathrm{C}(31)$ & $\mathrm{X}$ & $\mathrm{C}(30)$ & $\mathrm{H}(30)$ & $\mathrm{Y}$ & $\mathrm{R}$ & 2 & 3 & 14 & 4 & M & $C(50)$ \\
\hline $\mathrm{C}(31)$ & DUM14 & $Z$ & $\mathrm{C}(31)$ & $\mathrm{C}(30)$ & $\mathrm{Y}$ & $\mathrm{R}$ & 2 & 3 & 12 & 4 & MM2 & $\mathrm{C}(7)$ \\
\hline $\mathrm{C}(32)$ & $\mathrm{O}(40)$ & $Z$ & $\mathrm{C}(32)$ & $\mathrm{C}(31)$ & $\mathrm{X}$ & $\mathrm{R}$ & 2 & 3 & 11 & 4 & $3 \mathrm{M}$ & $\mathrm{C}(6)$ \\
\hline $\mathrm{C}(33)$ & $\mathrm{O}(39)$ & $\mathrm{X}$ & $\mathrm{C}(33)$ & $\mathrm{C}(32)$ & $\mathrm{Y}$ & $\mathrm{R}$ & 2 & 3 & 10 & 4 & M & $\mathrm{C}(3)$ \\
\hline $\mathrm{C}(34)$ & $\mathrm{O}(38)$ & $\mathrm{X}$ & $\mathrm{C}(34)$ & $\mathrm{C}(33)$ & $\mathrm{Y}$ & $\mathrm{R}$ & 2 & 3 & 10 & 4 & M & $\mathrm{C}(3)$ \\
\hline $\mathrm{C}(35)$ & $\mathrm{C}(34)$ & $Z$ & $\mathrm{C}(35)$ & $\mathrm{H}(35 \mathrm{~A})$ & Y & $\mathrm{R}$ & 2 & 3 & 16 & 4 & 3 & $C(55)$ \\
\hline$C(36)$ & $\mathrm{C}(32)$ & $Z$ & $\mathrm{C}(36)$ & $\mathrm{H}(36 \mathrm{~A})$ & $\mathrm{Y}$ & $\mathrm{R}$ & 2 & 3 & 16 & 4 & 3 & C(55) \\
\hline $\mathrm{C}(37)$ & $\mathrm{O}(40)$ & Z & $\mathrm{C}(37)$ & $\mathrm{H}(37 \mathrm{~A})$ & $\mathrm{X}$ & $\mathrm{R}$ & 2 & 3 & 18 & 4 & $3 \mathrm{M}$ & \\
\hline$C(60)$ & $\mathrm{C}(2)$ & $Z$ & $\mathrm{C}(60)$ & $\mathrm{H}(60 \mathrm{~A})$ & Y & $\mathrm{R}$ & 2 & 3 & 16 & 4 & 3 & C(55) \\
\hline $\mathrm{C}(61)$ & $C(4)$ & $Z$ & $\mathrm{C}(61)$ & $\mathrm{H}(61 \mathrm{~A})$ & $\mathrm{Y}$ & $\mathrm{R}$ & 2 & 3 & 16 & 4 & 3 & C(55) \\
\hline $\mathrm{C}(62)$ & $\mathrm{C}(6)$ & $Z$ & $\mathrm{C}(62)$ & $\mathrm{H}(62 \mathrm{~A})$ & $\mathrm{Y}$ & $\mathrm{R}$ & 2 & 3 & 16 & 4 & 3 & $C(55)$ \\
\hline $\mathrm{C}(63)$ & $\mathrm{C}(8)$ & $Z$ & $\mathrm{C}(63)$ & $\mathrm{H}(63 \mathrm{~A})$ & $\mathrm{Y}$ & $\mathrm{R}$ & 2 & 3 & 16 & 4 & 3 & C(55) \\
\hline$C(64)$ & $C(10)$ & $Z$ & $\mathrm{C}(64)$ & $\mathrm{H}(64 \mathrm{~A})$ & $\mathrm{Y}$ & $\mathrm{R}$ & 2 & 3 & 16 & 4 & 3 & C(55) \\
\hline $\mathrm{C}(65)$ & $\mathrm{C}(12)$ & $Z$ & $\mathrm{C}(65)$ & $\mathrm{H}(65 \mathrm{~A})$ & $\mathrm{Y}$ & $\mathrm{R}$ & 2 & 3 & 16 & 4 & 3 & $C(55)$ \\
\hline $\mathrm{C}(66)$ & DUM15 & $Z$ & $\mathrm{C}(66)$ & $\mathrm{C}(13)$ & $\mathrm{Y}$ & $\mathrm{R}$ & 2 & 3 & 12 & 4 & MM2 & $\mathrm{C}(7)$ \\
\hline $\mathrm{C}(67)$ & C(66) & Z & $\mathrm{C}(67)$ & $\mathrm{H}(67 \mathrm{~A})$ & $\mathrm{Y}$ & $\mathrm{R}$ & 2 & 3 & 16 & 4 & 3 & $C(55)$ \\
\hline $\mathrm{C}(68)$ & DUM16 & $Z$ & $\mathrm{C}(68)$ & $\mathrm{O}(25)$ & $\mathrm{Y}$ & $\mathrm{R}$ & 2 & 3 & 19 & 4 & MM2 & \\
\hline $\mathrm{C}(69)$ & $\mathrm{O}(26)$ & $\mathrm{X}$ & C(69) & $\mathrm{C}(70)$ & Y & $\mathrm{R}$ & 2 & 3 & 20 & 4 & M & \\
\hline $\mathrm{C}(70)$ & $\mathrm{O}(27)$ & $\mathrm{X}$ & $\mathrm{C}(70)$ & $\mathrm{C}(69)$ & Y & $\mathrm{R}$ & 2 & 3 & 20 & 4 & M & \\
\hline $\mathrm{C}(71)$ & $\mathrm{O}(27)$ & Z & $\mathrm{C}(71)$ & $\mathrm{H}(71 \mathrm{~A})$ & $\mathrm{X}$ & $\mathrm{R}$ & 2 & 3 & 18 & 4 & $3 \mathrm{M}$ & \\
\hline $\mathrm{H}(22)$ & $\mathrm{O}(22)$ & $Z$ & $\mathrm{H}(22)$ & C(6) & Y & $\mathrm{R}$ & 2 & 4 & 21 & 2 & 6 & \\
\hline $\mathrm{H}(23)$ & $\mathrm{O}(23)$ & Z & $\mathrm{H}(23)$ & $\mathrm{C}(11)$ & $\mathrm{Y}$ & $\mathrm{R}$ & 2 & 4 & 21 & 2 & 6 & \\
\hline $\mathrm{H}(24)$ & $\mathrm{O}(24)$ & Z & $\mathrm{H}(24)$ & $\mathrm{C}(12)$ & $\mathrm{Y}$ & $\mathrm{R}$ & 2 & 4 & 21 & 2 & 6 & \\
\hline H(59) & $\mathrm{O}(59)$ & $Z$ & H(59) & $\mathrm{C}(51)$ & Y & $\mathrm{R}$ & 2 & 4 & 21 & 2 & 6 & \\
\hline H(39) & $\mathrm{O}(39)$ & $Z$ & $\mathrm{H}(39)$ & $\mathrm{C}(33)$ & $\mathrm{Y}$ & $\mathrm{R}$ & 2 & 4 & 21 & 2 & 6 & \\
\hline $\mathrm{H}(2)$ & $\mathrm{C}(2)$ & Z & $\mathrm{H}(2)$ & $\mathrm{C}(1)$ & $\mathrm{Y}$ & $\mathrm{R}$ & 2 & 4 & 22 & 2 & 6 & \\
\hline $\mathrm{H}(3)$ & $\mathrm{C}(3)$ & $Z$ & $\mathrm{H}(3)$ & $\mathrm{O}(3)$ & Y & $\mathrm{R}$ & 2 & 4 & 23 & 2 & 6 & \\
\hline $\mathrm{H}(4)$ & $\mathrm{C}(4)$ & $Z$ & $\mathrm{H}(4)$ & $\mathrm{C}(3)$ & Y & $\mathrm{R}$ & 2 & 4 & 22 & 2 & 6 & $\mathrm{H}(2)$ \\
\hline $\mathrm{H}(5)$ & $\mathrm{C}(5)$ & $Z$ & $\mathrm{H}(5)$ & $\mathrm{O}(5)$ & $\mathrm{Y}$ & $\mathrm{R}$ & 2 & 4 & 23 & 2 & 6 & $\mathrm{H}(3)$ \\
\hline $\mathrm{H}(7 \mathrm{~A})$ & $\mathrm{C}(7)$ & $Z$ & $\mathrm{H}(7 \mathrm{~A})$ & $\mathrm{C}(6)$ & Y & $\mathrm{R}$ & 2 & 4 & 24 & 2 & 6 & \\
\hline $\mathrm{H}(7 \mathrm{~B})$ & $\mathrm{C}(7)$ & Z & $\mathrm{H}(7 \mathrm{~B})$ & $\mathrm{C}(6)$ & $\mathrm{Y}$ & $\mathrm{R}$ & 2 & 4 & 24 & 2 & 6 & $\mathrm{H}(7 \mathrm{~A})$ \\
\hline $\mathrm{H}(8)$ & $\mathrm{C}(8)$ & Z & $\mathrm{H}(8)$ & $\mathrm{C}(7)$ & $\mathrm{Y}$ & $\mathrm{R}$ & 2 & 4 & 22 & 2 & 6 & $\mathrm{H}(2)$ \\
\hline $\mathrm{H}(10)$ & $\mathrm{C}(10)$ & $Z$ & $\mathrm{H}(10)$ & $\mathrm{C}(9)$ & Y & $\mathrm{R}$ & 2 & 4 & 22 & 2 & 6 & $\mathrm{H}(2)$ \\
\hline $\mathrm{H}(11)$ & $\mathrm{C}(11)$ & $Z$ & $\mathrm{H}(11)$ & $\mathrm{O}(23)$ & $\mathrm{Y}$ & $\mathrm{R}$ & 2 & 4 & 23 & 2 & 6 & $\mathrm{H}(3)$ \\
\hline $\mathrm{H}(13)$ & $\mathrm{C}(13)$ & Z & $\mathrm{H}(13)$ & $\mathrm{O}(14)$ & $\mathrm{Y}$ & $\mathrm{R}$ & 2 & 4 & 23 & 2 & 6 & $\mathrm{H}(3)$ \\
\hline $\mathrm{H}(50)$ & $\mathrm{C}(50)$ & $Z$ & $\mathrm{H}(50)$ & $\mathrm{O}(5)$ & Y & $\mathrm{R}$ & 2 & 4 & 25 & 2 & 6 & \\
\hline $\mathrm{H}(51)$ & $\mathrm{C}(51)$ & Z & $\mathrm{H}(51)$ & O(59) & $\mathrm{Y}$ & $\mathrm{R}$ & 2 & 4 & 23 & 2 & 6 & $\mathrm{H}(3)$ \\
\hline
\end{tabular}


ATOM ATOMO AX1 ATOM1 ATOM2 AX2 R/L TP TBL KAP LMX SITESYM CHEMCON

\begin{tabular}{|c|c|c|c|c|c|c|c|c|c|c|c|c|}
\hline 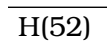 & $\mathrm{C}(52)$ & $Z$ & $\mathrm{H}(52)$ & $\mathrm{N}(2)$ & $\mathrm{Y}$ & $\mathrm{R}$ & 2 & 4 & 26 & 2 & 6 & \\
\hline $\mathrm{H}(53 \mathrm{~A})$ & $\mathrm{C}(53)$ & $Z$ & $\mathrm{H}(53 \mathrm{~A})$ & $\mathrm{C}(52)$ & Y & $\mathrm{R}$ & 2 & 4 & 24 & 2 & 6 & $\mathrm{H}(7 \mathrm{~A})$ \\
\hline $\mathrm{H}(53 \mathrm{~B})$ & C(53) & $Z$ & $\mathrm{H}(53 \mathrm{~B})$ & $\mathrm{C}(52)$ & Y & $\mathrm{R}$ & 2 & 4 & 24 & 2 & 6 & $\mathrm{H}(7 \mathrm{~A})$ \\
\hline $\mathrm{H}(54)$ & C(54) & $Z$ & $\mathrm{H}(54)$ & $\mathrm{O}(58)$ & Y & $\mathrm{R}$ & 2 & 4 & 23 & 2 & 6 & $\mathrm{H}(3)$ \\
\hline $\mathrm{H}(55 \mathrm{~A})$ & $\mathrm{C}(55)$ & Z & $\mathrm{H}(55 \mathrm{~A})$ & $\mathrm{C}(54)$ & Y & $\mathrm{R}$ & 2 & 4 & 27 & 2 & 6 & \\
\hline $\mathrm{H}(55 \mathrm{~B})$ & C(55) & Z & $\mathrm{H}(55 \mathrm{~B})$ & $\mathrm{C}(54)$ & Y & $\mathrm{R}$ & 2 & 4 & 27 & 2 & 6 & $\mathrm{H}(55 \mathrm{~A})$ \\
\hline $\mathrm{H}(55 \mathrm{C})$ & $C(55)$ & Z & $\mathrm{H}(55 \mathrm{C})$ & C(54) & $\mathrm{Y}$ & $\mathrm{R}$ & 2 & 4 & 27 & 2 & 6 & $\mathrm{H}(55 \mathrm{~A})$ \\
\hline $\mathrm{H}(56 \mathrm{~A})$ & $C(56)$ & Z & $\mathrm{H}(56 \mathrm{~A})$ & $\mathrm{N}(2)$ & $\mathrm{Y}$ & $\mathrm{R}$ & 2 & 4 & 28 & 2 & 6 & \\
\hline $\mathrm{H}(56 \mathrm{~B})$ & C(56) & $Z$ & $\mathrm{H}(56 \mathrm{~B})$ & $\mathrm{N}(2)$ & Y & $\mathrm{R}$ & 2 & 4 & 28 & 2 & 6 & $\mathrm{H}(56 \mathrm{~A})$ \\
\hline $\mathrm{H}(56 \mathrm{C})$ & C(56) & Z & $\mathrm{H}(56 \mathrm{C})$ & $\mathrm{N}(2)$ & $\mathrm{Y}$ & $\mathrm{R}$ & 2 & 4 & 28 & 2 & 6 & $\mathrm{H}(56 \mathrm{~A})$ \\
\hline $\mathrm{H}(57 \mathrm{~A})$ & C(57) & Z & $\mathrm{H}(57 \mathrm{~A})$ & $\mathrm{N}(2)$ & Y & $\mathrm{R}$ & 2 & 4 & 28 & 2 & 6 & $\mathrm{H}(56 \mathrm{~A})$ \\
\hline $\mathrm{H}(57 \mathrm{~B})$ & $\mathrm{C}(57)$ & $Z$ & $\mathrm{H}(57 \mathrm{~B})$ & $\mathrm{N}(2)$ & Y & $\mathrm{R}$ & 2 & 4 & 28 & 2 & 6 & $\mathrm{H}(56 \mathrm{~A})$ \\
\hline $\mathrm{H}(57 \mathrm{C})$ & C(57) & Z & $\mathrm{H}(57 \mathrm{C})$ & $\mathrm{N}(2)$ & $\mathrm{Y}$ & $\mathrm{R}$ & 2 & 4 & 28 & 2 & 6 & $\mathrm{H}(56 \mathrm{~A})$ \\
\hline $\mathrm{H}(30)$ & $\mathrm{C}(30)$ & Z & $\mathrm{H}(30)$ & $\mathrm{O}(3)$ & Y & $\mathrm{R}$ & 2 & 4 & 25 & 2 & 6 & $\mathrm{H}(50)$ \\
\hline $\mathrm{H}(31 \mathrm{~A})$ & $\mathrm{C}(31)$ & $Z$ & $\mathrm{H}(31 \mathrm{~A})$ & $\mathrm{C}(30)$ & Y & $\mathrm{R}$ & 2 & 4 & 24 & 2 & 6 & $\mathrm{H}(7 \mathrm{~A})$ \\
\hline $\mathrm{H}(31 \mathrm{~B})$ & $\mathrm{C}(31)$ & Z & $\mathrm{H}(31 \mathrm{~B})$ & $\mathrm{C}(30)$ & $\mathrm{Y}$ & $\mathrm{R}$ & 2 & 4 & 24 & 2 & 6 & $\mathrm{H}(7 \mathrm{~A})$ \\
\hline $\mathrm{H}(33)$ & $\mathrm{C}(33)$ & Z & $\mathrm{H}(33)$ & $\mathrm{O}(39)$ & Y & $\mathrm{R}$ & 2 & 4 & 23 & 2 & 6 & $\mathrm{H}(3)$ \\
\hline $\mathrm{H}(34)$ & $\mathrm{C}(34)$ & $Z$ & $\mathrm{H}(34)$ & $\mathrm{O}(38)$ & Y & $\mathrm{R}$ & 2 & 4 & 23 & 2 & 6 & $\mathrm{H}(3)$ \\
\hline $\mathrm{H}(35 \mathrm{~A})$ & $\mathrm{C}(35)$ & Z & $\mathrm{H}(35 \mathrm{~A})$ & $\mathrm{C}(34)$ & Y & $\mathrm{R}$ & 2 & 4 & 27 & 2 & 6 & $\mathrm{H}(55 \mathrm{~A})$ \\
\hline $\mathrm{H}(35 \mathrm{~B})$ & $\mathrm{C}(35)$ & $Z$ & $\mathrm{H}(35 \mathrm{~B})$ & $\mathrm{C}(34)$ & $\mathrm{Y}$ & $\mathrm{R}$ & 2 & 4 & 27 & 2 & 6 & $\mathrm{H}(55 \mathrm{~A})$ \\
\hline $\mathrm{H}(35 \mathrm{C})$ & $\mathrm{C}(35)$ & $Z$ & $\mathrm{H}(35 \mathrm{C})$ & $\mathrm{C}(34)$ & Y & $\mathrm{R}$ & 2 & 4 & 27 & 2 & 6 & $\mathrm{H}(55 \mathrm{~A})$ \\
\hline $\mathrm{H}(36 \mathrm{~A})$ & $\mathrm{C}(36)$ & Z & $\mathrm{H}(36 \mathrm{~A})$ & $\mathrm{C}(32)$ & $\mathrm{Y}$ & $\mathrm{R}$ & 2 & 4 & 27 & 2 & 6 & $\mathrm{H}(55 \mathrm{~A})$ \\
\hline $\mathrm{H}(36 \mathrm{~B})$ & $\mathrm{C}(36)$ & $Z$ & $\mathrm{H}(36 \mathrm{~B})$ & $\mathrm{C}(32)$ & $\mathrm{Y}$ & $\mathrm{R}$ & 2 & 4 & 27 & 2 & 6 & $\mathrm{H}(55 \mathrm{~A})$ \\
\hline $\mathrm{H}(36 \mathrm{C})$ & $\mathrm{C}(36)$ & $Z$ & $\mathrm{H}(36 \mathrm{C})$ & $\mathrm{C}(32)$ & Y & $\mathrm{R}$ & 2 & 4 & 27 & 2 & 6 & $\mathrm{H}(55 \mathrm{~A})$ \\
\hline $\mathrm{H}(37 \mathrm{~A})$ & $\mathrm{C}(37)$ & Z & $\mathrm{H}(37 \mathrm{~A})$ & $\mathrm{O}(40)$ & Y & $\mathrm{R}$ & 2 & 4 & 29 & 2 & 6 & \\
\hline $\mathrm{H}(37 \mathrm{~B})$ & $\mathrm{C}(37)$ & $Z$ & $\mathrm{H}(37 \mathrm{~B})$ & $\mathrm{O}(40)$ & Y & $\mathrm{R}$ & 2 & 4 & 29 & 2 & 6 & $\mathrm{H}(37 \mathrm{~A})$ \\
\hline $\mathrm{H}(37 \mathrm{C})$ & $\mathrm{C}(37)$ & $Z$ & $\mathrm{H}(37 \mathrm{C})$ & $\mathrm{O}(40)$ & Y & $\mathrm{R}$ & 2 & 4 & 29 & 2 & 6 & $\mathrm{H}(37 \mathrm{~A})$ \\
\hline $\mathrm{H}(60 \mathrm{~A})$ & $\mathrm{C}(60)$ & Z & $\mathrm{H}(60 \mathrm{~A})$ & $\mathrm{C}(2)$ & Y & $\mathrm{R}$ & 2 & 4 & 27 & 2 & 6 & $\mathrm{H}(55 \mathrm{~A})$ \\
\hline $\mathrm{H}(60 \mathrm{~B})$ & $\mathrm{C}(60)$ & Z & $\mathrm{H}(60 \mathrm{~B})$ & $\mathrm{C}(2)$ & Y & $\mathrm{R}$ & 2 & 4 & 27 & 2 & 6 & $\mathrm{H}(55 \mathrm{~A})$ \\
\hline $\mathrm{H}(60 \mathrm{C})$ & $\mathrm{C}(60)$ & $Z$ & $\mathrm{H}(60 \mathrm{C})$ & $\mathrm{C}(2)$ & Y & $\mathrm{R}$ & 2 & 4 & 27 & 2 & 6 & $\mathrm{H}(55 \mathrm{~A})$ \\
\hline $\mathrm{H}(61 \mathrm{~A})$ & $\mathrm{C}(61)$ & Z & $\mathrm{H}(61 \mathrm{~A})$ & $\mathrm{C}(4)$ & Y & $\mathrm{R}$ & 2 & 4 & 27 & 2 & 6 & $\mathrm{H}(55 \mathrm{~A})$ \\
\hline $\mathrm{H}(61 \mathrm{~B})$ & $\mathrm{C}(61)$ & Z & $\mathrm{H}(61 \mathrm{~B})$ & $\mathrm{C}(4)$ & Y & $\mathrm{R}$ & 2 & 4 & 27 & 2 & 6 & $\mathrm{H}(55 \mathrm{~A})$ \\
\hline $\mathrm{H}(61 \mathrm{C})$ & $\mathrm{C}(61)$ & $Z$ & $\mathrm{H}(61 \mathrm{C})$ & $\mathrm{C}(4)$ & Y & $\mathrm{R}$ & 2 & 4 & 27 & 2 & 6 & $\mathrm{H}(55 \mathrm{~A})$ \\
\hline $\mathrm{H}(62 \mathrm{~A})$ & $\mathrm{C}(62)$ & Z & $\mathrm{H}(62 \mathrm{~A})$ & $\mathrm{C}(6)$ & Y & $\mathrm{R}$ & 2 & 4 & 27 & 2 & 6 & $\mathrm{H}(55 \mathrm{~A})$ \\
\hline $\mathrm{H}(62 \mathrm{~B})$ & $\mathrm{C}(62)$ & $Z$ & $\mathrm{H}(62 \mathrm{~B})$ & $\mathrm{C}(6)$ & Y & $\mathrm{R}$ & 2 & 4 & 27 & 2 & 6 & $\mathrm{H}(55 \mathrm{~A})$ \\
\hline $\mathrm{H}(62 \mathrm{C})$ & $\mathrm{C}(62)$ & $Z$ & $\mathrm{H}(62 \mathrm{C})$ & $\mathrm{C}(6)$ & Y & $\mathrm{R}$ & 2 & 4 & 27 & 2 & 6 & $\mathrm{H}(55 \mathrm{~A})$ \\
\hline $\mathrm{H}(63 \mathrm{~A})$ & $\mathrm{C}(63)$ & Z & $\mathrm{H}(63 \mathrm{~A})$ & $\mathrm{C}(8)$ & Y & $\mathrm{R}$ & 2 & 4 & 27 & 2 & 6 & $\mathrm{H}(55 \mathrm{~A})$ \\
\hline $\mathrm{H}(63 \mathrm{~B})$ & $\mathrm{C}(63)$ & $Z$ & $\mathrm{H}(63 \mathrm{~B})$ & $\mathrm{C}(8)$ & Y & $\mathrm{R}$ & 2 & 4 & 27 & 2 & 6 & $\mathrm{H}(55 \mathrm{~A})$ \\
\hline $\mathrm{H}(63 \mathrm{C})$ & $\mathrm{C}(63)$ & $Z$ & $\mathrm{H}(63 \mathrm{C})$ & $\mathrm{C}(8)$ & Y & $\mathrm{R}$ & 2 & 4 & 27 & 2 & 6 & $\mathrm{H}(55 \mathrm{~A})$ \\
\hline $\mathrm{H}(64 \mathrm{~A})$ & $\mathrm{C}(64)$ & $Z$ & $\mathrm{H}(64 \mathrm{~A})$ & $\mathrm{C}(10)$ & Y & $\mathrm{R}$ & 2 & 4 & 27 & 2 & 6 & $\mathrm{H}(55 \mathrm{~A})$ \\
\hline $\mathrm{H}(64 \mathrm{~B})$ & $\mathrm{C}(64)$ & $Z$ & $\mathrm{H}(64 \mathrm{~B})$ & $\mathrm{C}(10)$ & Y & $\mathrm{R}$ & 2 & 4 & 27 & 2 & 6 & $\mathrm{H}(55 \mathrm{~A})$ \\
\hline $\mathrm{H}(64 \mathrm{C})$ & $\mathrm{C}(64)$ & $Z$ & $\mathrm{H}(64 \mathrm{C})$ & $\mathrm{C}(10)$ & Y & $\mathrm{R}$ & 2 & 4 & 27 & 2 & 6 & $\mathrm{H}(55 \mathrm{~A})$ \\
\hline $\mathrm{H}(65 \mathrm{~A})$ & $\mathrm{C}(65)$ & $Z$ & $\mathrm{H}(65 \mathrm{~A})$ & $\mathrm{C}(12)$ & Y & $\mathrm{R}$ & 2 & 4 & 27 & 2 & 6 & $\mathrm{H}(55 \mathrm{~A})$ \\
\hline $\mathrm{H}(65 \mathrm{~B})$ & $\mathrm{C}(65)$ & $Z$ & $\mathrm{H}(65 \mathrm{~B})$ & $\mathrm{C}(12)$ & Y & $\mathrm{R}$ & 2 & 4 & 27 & 2 & 6 & $\mathrm{H}(55 \mathrm{~A})$ \\
\hline $\mathrm{H}(65 \mathrm{C})$ & $\mathrm{C}(65)$ & $Z$ & $\mathrm{H}(65 \mathrm{C})$ & $\mathrm{C}(12)$ & Y & $\mathrm{R}$ & 2 & 4 & 27 & 2 & 6 & $\mathrm{H}(55 \mathrm{~A})$ \\
\hline $\mathrm{H}(66 \mathrm{~A})$ & $\mathrm{C}(66)$ & $Z$ & $\mathrm{H}(66 \mathrm{~A})$ & $\mathrm{C}(13)$ & Y & $\mathrm{R}$ & 2 & 4 & 24 & 2 & 6 & $\mathrm{H}(7 \mathrm{~A})$ \\
\hline $\mathrm{H}(66 \mathrm{~B})$ & C(66) & $Z$ & $\mathrm{H}(66 \mathrm{~B})$ & $\mathrm{C}(13)$ & $\mathrm{Y}$ & $\mathrm{R}$ & 2 & 4 & 24 & 2 & 6 & $\mathrm{H}(7 \mathrm{~A})$ \\
\hline $\mathrm{H}(67 \mathrm{~A})$ & $\mathrm{C}(67)$ & Z & $\mathrm{H}(67 \mathrm{~A})$ & $\mathrm{C}(66)$ & Y & $\mathrm{R}$ & 2 & 4 & 27 & 2 & 6 & $\mathrm{H}(55 \mathrm{~A})$ \\
\hline $\mathrm{H}(67 \mathrm{~B})$ & $\mathrm{C}(67)$ & $Z$ & $\mathrm{H}(67 \mathrm{~B})$ & $\mathrm{C}(66)$ & Y & $\mathrm{R}$ & 2 & 4 & 27 & 2 & 6 & $\mathrm{H}(55 \mathrm{~A})$ \\
\hline
\end{tabular}


ATOM ATOMO AX1 ATOM1 ATOM2 AX2 R/L TP TBL KAP LMX SITESYM CHEMCON

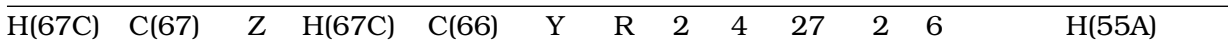

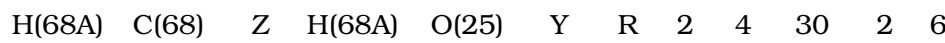

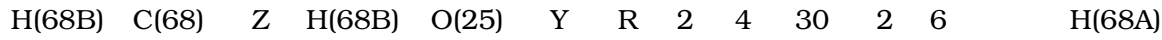

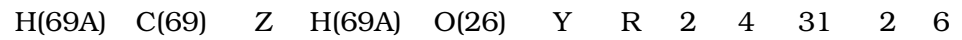

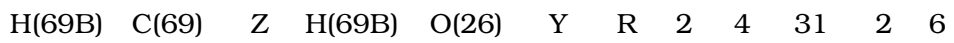

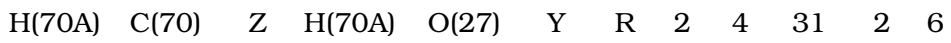

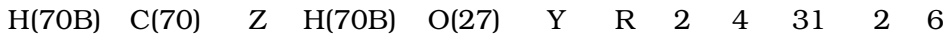

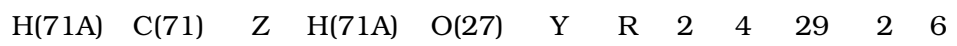

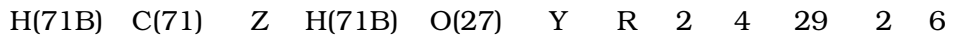

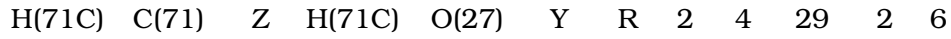

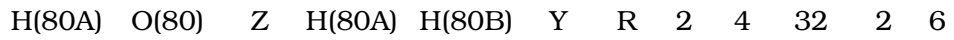

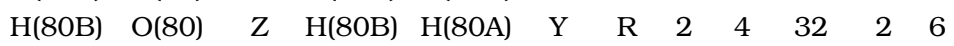


A. Tabellen 


\section{A.1.2 Ergebnisse des Hishfeld-Tests (DMSDA) der GMV}

\begin{tabular}{|c|c|c|c|}
\hline Atom 1 & Atom 2 & $\begin{array}{c}\text { Bindungslänge } \\
{[\AA \AA]}\end{array}$ & $\begin{array}{r}\text { DMSDA } \\
{\left[10^{-4} \AA^{2}\right]}\end{array}$ \\
\hline $\mathrm{O}(14)$ & $\mathrm{C}(1)$ & 1,3395 & -8 \\
\hline $\mathrm{O}(14)$ & $C(13)$ & 1,4574 & 1 \\
\hline $\mathrm{O}(21)$ & $\mathrm{C}(1)$ & 1,2110 & -2 \\
\hline $\mathrm{O}(22)$ & $C(6)$ & 1,4264 & -2 \\
\hline $\mathrm{O}(23)$ & $C(11)$ & 1,4297 & -1 \\
\hline $\mathrm{O}(24)$ & $C(12)$ & 1,4294 & 2 \\
\hline $\mathrm{O}(25)$ & $\mathrm{N}(1)$ & 1,4292 & -3 \\
\hline $\mathrm{O}(25)$ & $\mathrm{C}(68)$ & 1,4084 & 0 \\
\hline $\mathrm{O}(26)$ & $\mathrm{C}(68)$ & 1,4036 & -1 \\
\hline $\mathrm{O}(26)$ & $\mathrm{C}(69)$ & 1,4192 & 18 \\
\hline $\mathrm{O}(27)$ & $\mathrm{C}(70)$ & 1,4093 & 19 \\
\hline $\mathrm{O}(27)$ & $\mathrm{C}(71)$ & 1,4197 & 9 \\
\hline $\mathrm{O}(5)$ & $\mathrm{C}(5)$ & 1,4375 & 3 \\
\hline $\mathrm{O}(5)$ & $C(50)$ & 1,3923 & 4 \\
\hline $\mathrm{O}(58)$ & $C(50)$ & 1,4183 & 1 \\
\hline $\mathrm{O}(58)$ & $\mathrm{C}(54)$ & 1,4357 & 7 \\
\hline $\mathrm{O}(59)$ & $\mathrm{C}(51)$ & 1,4185 & 2 \\
\hline $\mathrm{O}(3)$ & $\mathrm{C}(3)$ & 1,4302 & 4 \\
\hline $\mathrm{O}(3)$ & $\mathrm{C}(30)$ & 1,4190 & 2 \\
\hline $\mathrm{O}(38)$ & $\mathrm{C}(30)$ & 1,4100 & 2 \\
\hline $\mathrm{O}(38$ & $\mathrm{C}(34)$ & 1,4367 & 0 \\
\hline $\mathrm{O}(39)$ & $\mathrm{C}(33)$ & 1,4189 & 0 \\
\hline $\mathrm{O}(40)$ & $\mathrm{C}(32)$ & 1,4300 & 1 \\
\hline $\mathrm{O}(40)$ & $\mathrm{C}(37)$ & 1,4155 & 15 \\
\hline $\mathrm{N}(2)$ & $\mathrm{C}(52)$ & 1,4834 & -1 \\
\hline $\mathrm{N}(2)$ & $C(56)$ & 1,4677 & 0 \\
\hline $\mathrm{N}(2)$ & $\mathrm{C}(57)$ & 1,4653 & 1 \\
\hline $\mathrm{N}(1)$ & $\mathrm{C}(9)$ & 1,2785 & 1 \\
\hline$C(1)$ & $\mathrm{C}(2)$ & 1,5171 & 1 \\
\hline$C(2)$ & $\mathrm{C}(3)$ & 1,5502 & -4 \\
\hline $\mathrm{C}(2)$ & $C(60)$ & 1,5354 & 1 \\
\hline$C(3)$ & $\mathrm{C}(4)$ & 1,5431 & -2 \\
\hline $\mathrm{C}(4)$ & $\mathrm{C}(5)$ & 1,5574 & 0 \\
\hline $\mathrm{C}(4)$ & $\mathrm{C}(61)$ & 1,5282 & -2 \\
\hline
\end{tabular}




\begin{tabular}{|c|c|c|c|}
\hline Atom 1 & Atom 2 & $\begin{array}{c}\text { Bindungslänge } \\
{[\AA ̊ \AA]}\end{array}$ & $\begin{array}{c}\text { DMSDA } \\
{\left[10^{-4} \AA^{2}\right]}\end{array}$ \\
\hline $\mathrm{C}(5)$ & $\mathrm{C}(6)$ & 1,5572 & 1 \\
\hline $\mathrm{C}(6)$ & $\mathrm{C}(7)$ & 1,5349 & 1 \\
\hline $\mathrm{C}(6)$ & $\mathrm{C}(62)$ & 1,5279 & 3 \\
\hline $\mathrm{C}(7)$ & $\mathrm{C}(8)$ & 1,5386 & 4 \\
\hline $\mathrm{C}(8)$ & $\mathrm{C}(9)$ & 1,5145 & -2 \\
\hline $\mathrm{C}(8)$ & $\mathrm{C}(63)$ & 1,5403 & 5 \\
\hline $\mathrm{C}(9)$ & $\mathrm{C}(10)$ & 1,5277 & 0 \\
\hline$C(10)$ & $\mathrm{C}(11)$ & 1,5449 & -5 \\
\hline$C(10)$ & $\mathrm{C}(64)$ & 1,5355 & -1 \\
\hline $\mathrm{C}(11)$ & $\mathrm{C}(12)$ & 1,5541 & 0 \\
\hline $\mathrm{C}(12)$ & $C(13)$ & 1,5534 & 3 \\
\hline $\mathrm{C}(12)$ & $\mathrm{C}(65)$ & 1,5208 & 2 \\
\hline $\mathrm{C}(13)$ & $\mathrm{C}(66)$ & 1,5213 & -1 \\
\hline$C(50)$ & $\mathrm{C}(51)$ & 1,5334 & 1 \\
\hline $\mathrm{C}(51)$ & $C(52)$ & 1,5371 & 0 \\
\hline $\mathrm{C}(52)$ & $\mathrm{C}(53)$ & 1,5314 & 1 \\
\hline $\mathrm{C}(53)$ & $C(54)$ & 1,5212 & 0 \\
\hline $\mathrm{C}(54)$ & $C(55)$ & 1,5150 & -2 \\
\hline $\mathrm{C}(30)$ & $\mathrm{C}(31)$ & 1,5231 & 0 \\
\hline $\mathrm{C}(31)$ & $\mathrm{C}(32)$ & 1,5369 & -1 \\
\hline $\mathrm{C}(32)$ & $\mathrm{C}(33)$ & 1,5364 & 5 \\
\hline $\mathrm{C}(32)$ & $\mathrm{C}(36)$ & 1,5270 & 3 \\
\hline $\mathrm{C}(33)$ & $\mathrm{C}(34)$ & 1,5295 & 1 \\
\hline $\mathrm{C}(34)$ & $\mathrm{C}(35)$ & 1,5154 & 3 \\
\hline $\mathrm{C}(66)$ & $\mathrm{C}(67)$ & 1,5256 & -2 \\
\hline $\mathrm{C}(69)$ & $\mathrm{C}(70)$ & 1,5079 & 10 \\
\hline
\end{tabular}


A.1.3 Bindungkritische Punkte der GMV von Roxithromycin

\begin{tabular}{|lrrc|}
\hline \multicolumn{1}{|c}{ Bindung } & $\rho\left(\mathbf{r}_{b c p}\right)$ & $\nabla^{2} \rho\left(\mathbf{r}_{\mathrm{bcp}}\right)$ & $\epsilon$ \\
\hline $\mathrm{O}(14)-\mathrm{C}(1)$ & 2,269 & $-23,450$ & 0,14 \\
$\mathrm{O}(14)-\mathrm{C}(13)$ & 1,674 & $-7,024$ & 0,04 \\
$\mathrm{O}(21)-\mathrm{C}(1)$ & 2,925 & $-31,246$ & 0,06 \\
$\mathrm{O}(22)-\mathrm{C}(6)$ & 1,768 & $-7,783$ & 0,01 \\
$\mathrm{O}(22)-\mathrm{H}(22)$ & 2,229 & $-38,702$ & 0,04 \\
$\mathrm{O}(23)-\mathrm{C}(11)$ & 1,714 & $-6,502$ & 0,06 \\
\hline
\end{tabular}




\begin{tabular}{|lrrc|}
\hline \multicolumn{1}{|c}{ Bindung } & $\rho\left(\mathbf{r}_{b c p}\right)$ & \multicolumn{1}{c|}{$\rho\left(\mathbf{r}_{\mathrm{bcp}}\right)$} & $\epsilon$ \\
\hline $\mathrm{O}(23)-\mathrm{H}(23)$ & 2,336 & $-44,191$ & 0,01 \\
$\mathrm{O}(24)-\mathrm{C}(12)$ & 1,847 & $-12,257$ & 0,04 \\
$\mathrm{O}(24)-\mathrm{H}(24)$ & 2,484 & $-49,722$ & 0,03 \\
$\mathrm{O}(25)-\mathrm{N}(1)$ & 1,890 & 8,779 & 0,04 \\
$\mathrm{O}(25)-\mathrm{C}(68)$ & 1,877 & $-9,989$ & 0,03 \\
$\mathrm{O}(26)-\mathrm{C}(68)$ & 1,895 & $-11,158$ & 0,10 \\
$\mathrm{O}(26)-\mathrm{C}(69)$ & 1,816 & $-12,388$ & 0,03 \\
$\mathrm{O}(27)-\mathrm{C}(70)$ & 1,794 & $-10,662$ & 0,03 \\
$\mathrm{O}(27)-\mathrm{C}(71)$ & 1,767 & $-10,260$ & 0,02 \\
$\mathrm{O}(5)-\mathrm{C}(5)$ & 1,731 & $-8,687$ & 0,04 \\
$\mathrm{O}(5)-\mathrm{C}(50)$ & 1,931 & $-15,134$ & 0,02 \\
$\mathrm{O}(58)-\mathrm{C}(50)$ & 1,857 & $-11,998$ & 0,06 \\
$\mathrm{O}(58)-\mathrm{C}(54)$ & 1,741 & $-8,746$ & 0,03 \\
$\mathrm{O}(59)-\mathrm{C}(51)$ & 1,783 & $-9,894$ & 0,02 \\
$\mathrm{O}(59)-\mathrm{H}(59)$ & 2,396 & $-31,448$ & 0,00 \\
$\mathrm{O}(3)-\mathrm{C}(3)$ & 1,749 & $-9,326$ & 0,04 \\
$\mathrm{O}(3)-\mathrm{C}(30)$ & 1,859 & $-12,430$ & 0,03 \\
$\mathrm{O}(38)-\mathrm{C}(30)$ & 1,880 & $-13,145$ & 0,05 \\
$\mathrm{O}(38)-\mathrm{C}(34)$ & 1,734 & $-8,606$ & 0,04 \\
$\mathrm{O}(39)-\mathrm{C}(33)$ & 1,781 & $-9,920$ & 0,06 \\
$\mathrm{O}(39)-\mathrm{H}(39)$ & 2,436 & $-35,890$ & 0,01 \\
$\mathrm{O}(40)-\mathrm{C}(32)$ & 1,828 & $-11,199$ & 0,03 \\
$\mathrm{O}(40)-\mathrm{C}(37)$ & 1,850 & $-14,835$ & 0,03 \\
$\mathrm{O}(80)-\mathrm{H}(80 A)$ & 2,462 & $-35,193$ & 0,02 \\
$\mathrm{O}(80)-\mathrm{H}(80 B)$ & 2,492 & $-33,180$ & 0,03 \\
$\mathrm{~N}(2)-\mathrm{C}(52)$ & 1,766 & $-9,071$ & 0,08 \\
$\mathrm{~N}(2)-\mathrm{C}(56)$ & 1,798 & $-9,457$ & 0,07 \\
$\mathrm{~N}(2)-\mathrm{C}(57)$ & 1,812 & $-9,983$ & 0,06 \\
$\mathrm{~N}(1)-\mathrm{C}(9)$ & 2,453 & $-17,044$ & 0,48 \\
$\mathrm{C}(1)-\mathrm{C}(2)$ & 1,724 & $-12,078$ & 0,13 \\
$\mathrm{C}(2)-\mathrm{C}(3)$ & 1,621 & $-10,120$ & 0,03 \\
$\mathrm{C}(2)-\mathrm{C}(60)$ & 1,594 & $-8,822$ & 0,01 \\
$\mathrm{C}(2)-\mathrm{H}(2)$ & 1,911 & $-21,474$ & 0,00 \\
$\mathrm{C}(3)-\mathrm{C}(4)$ & 1,628 & $-10,521$ & 0,02 \\
$\mathrm{C}(3)-\mathrm{H}(3)$ & 1,909 & $-21,066$ & 0,02 \\
\hline & & &
\end{tabular}




\begin{tabular}{|lrrc|}
\hline \multicolumn{1}{|c}{ Bindung } & $\rho\left(\mathbf{r}_{b c p}\right)$ & $\nabla^{2} \rho\left(\mathbf{r}_{\mathrm{bcp}}\right)$ & $\epsilon$ \\
\hline $\mathrm{C}(4)-\mathrm{C}(5)$ & 1,603 & $-9,896$ & 0,04 \\
$\mathrm{C}(4)-\mathrm{C}(61)$ & 1,610 & $-9,285$ & 0,00 \\
$\mathrm{C}(4)-\mathrm{H}(4)$ & 1,912 & $-21,409$ & 0,00 \\
$\mathrm{C}(5)-\mathrm{C}(6)$ & 1,645 & $-11,095$ & 0,08 \\
$\mathrm{C}(5)-\mathrm{H}(5)$ & 1,910 & $-21,075$ & 0,02 \\
$\mathrm{C}(6)-\mathrm{C}(7)$ & 1,650 & $-10,576$ & 0,05 \\
$\mathrm{C}(6)-\mathrm{C}(62)$ & 1,670 & $-10,687$ & 0,06 \\
$\mathrm{C}(7)-\mathrm{C}(8)$ & 1,587 & $-9,131$ & 0,01 \\
$\mathrm{C}(7)-\mathrm{H}(7 \mathrm{~A})$ & 1,851 & $-18,440$ & 0,02 \\
$\mathrm{C}(7)-\mathrm{H}(7 \mathrm{~B})$ & 1,859 & $-18,585$ & 0,02 \\
$\mathrm{C}(8)-\mathrm{C}(9)$ & 1,649 & $-9,447$ & 0,06 \\
$\mathrm{C}(8)-\mathrm{C}(63)$ & 1,586 & $-8,699$ & 0,01 \\
$\mathrm{C}(8)-\mathrm{H}(8)$ & 1,909 & $-21,471$ & 0,00 \\
$\mathrm{C}(9)-\mathrm{C}(10)$ & 1,613 & $-8,692$ & 0,05 \\
$\mathrm{C}(10)-\mathrm{C}(11)$ & 1,634 & $-10,456$ & 0,04 \\
$\mathrm{C}(10)-\mathrm{C}(64)$ & 1,594 & $-8,956$ & 0,00 \\
$\mathrm{C}(10)-\mathrm{H}(10)$ & 1,912 & $-21,438$ & 0,00 \\
$\mathrm{C}(11)-\mathrm{C}(12)$ & 1,647 & $-11,066$ & 0,04 \\
$\mathrm{C}(11)-\mathrm{H}(11)$ & 1,912 & $-21,100$ & 0,03 \\
$\mathrm{C}(12)-\mathrm{C}(13)$ & 1,662 & $-11,146$ & 0,08 \\
$\mathrm{C}(12)-\mathrm{C}(65)$ & 1,686 & $-10,907$ & 0,06 \\
$\mathrm{C}(13)-\mathrm{C}(66)$ & 1,685 & $-11,422$ & 0,03 \\
$\mathrm{C}(13)-\mathrm{H}(13)$ & 1,911 & $-21,109$ & 0,03 \\
$\mathrm{C}(50)-\mathrm{C}(51)$ & 1,740 & $-13,319$ & 0,02 \\
$\mathrm{C}(50)-\mathrm{H}(50)$ & 1,946 & $-22,605$ & 0,04 \\
$\mathrm{C}(51)-\mathrm{C}(52)$ & 1,724 & $-12,608$ & 0,04 \\
$\mathrm{C}(51)-\mathrm{H}(51)$ & 1,905 & $-20,815$ & 0,03 \\
$\mathrm{C}(52)-\mathrm{C}(53)$ & 1,694 & $-11,441$ & 0,01 \\
$\mathrm{C}(52)-\mathrm{H}(52)$ & 1,829 & $-18,817$ & 0,07 \\
$\mathrm{C}(53)-\mathrm{C}(54)$ & 1,707 & $-11,493$ & 0,04 \\
$\mathrm{C}(53)-\mathrm{H}(53 \mathrm{~A})$ & 1,856 & $-18,559$ & 0,02 \\
$\mathrm{C}(53)-\mathrm{H}(53 \mathrm{~B})$ & 1,859 & $-18,587$ & 0,01 \\
$\mathrm{C}(54)-\mathrm{C}(55)$ & 1,703 & $-11,506$ & 0,03 \\
$\mathrm{C}(54)-\mathrm{H}(54)$ & 1,902 & $-20,761$ & 0,02 \\
$\mathrm{C}(55)-\mathrm{H}(55 \mathrm{~A})$ & 1,833 & $-18,262$ & 0,02 \\
\hline
\end{tabular}




\begin{tabular}{|cccc|}
\hline Bindung & $\rho\left(\mathbf{r}_{b c p}\right)$ & $\nabla^{2} \rho\left(\mathbf{r}_{\mathrm{bcp}}\right)$ & $\epsilon$ \\
\hline $\mathrm{C}(55)-\mathrm{H}(55 \mathrm{~B})$ & 1,827 & $-18,131$ & 0,01 \\
$\mathrm{C}(55)-\mathrm{H}(55 \mathrm{C})$ & 1,827 & $-18,152$ & 0,03 \\
$\mathrm{C}(56)-\mathrm{H}(56 \mathrm{~A})$ & 1,874 & $-20,163$ & 0,01 \\
$\mathrm{C}(56)-\mathrm{H}(56 \mathrm{~B})$ & 1,876 & $-20,371$ & 0,01 \\
$\mathrm{C}(56)-\mathrm{H}(56 \mathrm{C})$ & 1,881 & $-20,484$ & 0,02 \\
$\mathrm{C}(57)-\mathrm{H}(57 \mathrm{~A})$ & 1,883 & $-20,297$ & 0,02 \\
$\mathrm{C}(57)-\mathrm{H}(57 \mathrm{~B})$ & 1,851 & $-19,394$ & 0,02 \\
$\mathrm{C}(57)-\mathrm{H}(57 \mathrm{C})$ & 1,879 & $-20,517$ & 0,02 \\
$\mathrm{C}(30)-\mathrm{C}(31)$ & 1,701 & $-12,349$ & 0,01 \\
$\mathrm{C}(30)-\mathrm{H}(30)$ & 1,932 & $-22,084$ & 0,04 \\
$\mathrm{C}(31)-\mathrm{C}(32)$ & 1,651 & $-10,537$ & 0,07 \\
$\mathrm{C}(31)-\mathrm{H}(31 \mathrm{~A})$ & 1,856 & $-18,544$ & 0,02 \\
$\mathrm{C}(31)-\mathrm{H}(31 \mathrm{~B})$ & 1,858 & $-18,607$ & 0,01 \\
$\mathrm{C}(32)-\mathrm{C}(33)$ & 1,712 & $-12,004$ & 0,04 \\
$\mathrm{C}(32)-\mathrm{C}(36)$ & 1,671 & $-10,613$ & 0,06 \\
$\mathrm{C}(33)-\mathrm{C}(34)$ & 1,735 & $-12,817$ & 0,06 \\
$\mathrm{C}(33)-\mathrm{H}(33)$ & 1,910 & $-20,969$ & 0,03 \\
$\mathrm{C}(34)-\mathrm{C}(35)$ & 1,702 & $-11,403$ & 0,02 \\
$\mathrm{C}(34)-\mathrm{H}(34)$ & 1,911 & $-21,073$ & 0,02 \\
$\mathrm{C}(35)-\mathrm{H}(35 \mathrm{~A})$ & 1,830 & $-18,222$ & 0,03 \\
$\mathrm{C}(35)-\mathrm{H}(35 \mathrm{~B})$ & 1,827 & $-18,194$ & 0,03 \\
$\mathrm{C}(35)-\mathrm{H}(35 \mathrm{C})$ & 1,819 & $-17,700$ & 0,00 \\
$\mathrm{C}(36)-\mathrm{H}(36 \mathrm{~A})$ & 1,830 & $-18,249$ & 0,02 \\
$\mathrm{C}(36)-\mathrm{H}(36 \mathrm{~B})$ & 1,832 & $-18,195$ & 0,02 \\
$\mathrm{C}(36)-\mathrm{H}(36 \mathrm{C})$ & 1,821 & $-18,106$ & 0,01 \\
$\mathrm{C}(37)-\mathrm{H}(37 \mathrm{~A})$ & 1,886 & $-21,169$ & 0,05 \\
$\mathrm{C}(37)-\mathrm{H}(37 \mathrm{~B})$ & 1,871 & $-20,851$ & 0,05 \\
$\mathrm{C}(37)-\mathrm{H}(37 \mathrm{C})$ & 1,873 & $-20,957$ & 0,05 \\
$\mathrm{C}(60)-\mathrm{H}(60 A)$ & 1,820 & $-17,897$ & 0,01 \\
$\mathrm{C}(60)-\mathrm{H}(60 B)$ & 1,814 & $-17,908$ & 0,03 \\
$\mathrm{C}(60)-\mathrm{H}(60 \mathrm{C})$ & 1,813 & $-17,591$ & 0,02 \\
$\mathrm{C}(61)-\mathrm{H}(61 \mathrm{~A})$ & 1,828 & $-18,139$ & 0,01 \\
$\mathrm{C}(61)-\mathrm{H}(61 \mathrm{~B})$ & 1,830 & $-18,225$ & 0,03 \\
$\mathrm{C}(61)-\mathrm{H}(61 \mathrm{C})$ & 1,830 & $-18,208$ & 0,03 \\
$\mathrm{C}(62)-\mathrm{H}(62 \mathrm{~A})$ & 1,832 & $-18,243$ & 0,02 \\
\hline & & & \\
\end{tabular}




\begin{tabular}{|lrrc|}
\hline Bindung & $\rho\left(\mathbf{r}_{b c p}\right)$ & $\nabla^{2} \rho\left(\mathbf{r}_{\mathrm{bcp}}\right)$ & $\epsilon$ \\
\hline $\mathrm{C}(62)-\mathrm{H}(62 \mathrm{~B})$ & 1,831 & $-18,218$ & 0,02 \\
$\mathrm{C}(62)-\mathrm{H}(62 \mathrm{C})$ & 1,831 & $-18,311$ & 0,02 \\
$\mathrm{C}(63)-\mathrm{H}(63 \mathrm{~A})$ & 1,824 & $-18,122$ & 0,01 \\
$\mathrm{C}(63)-\mathrm{H}(63 \mathrm{~B})$ & 1,826 & $-18,202$ & 0,02 \\
$\mathrm{C}(63)-\mathrm{H}(63 \mathrm{C})$ & 1,823 & $-18,107$ & 0,01 \\
$\mathrm{C}(64)-\mathrm{H}(64 \mathrm{~A})$ & 1,828 & $-18,159$ & 0,01 \\
$\mathrm{C}(64)-\mathrm{H}(64 \mathrm{~B})$ & 1,829 & $-18,278$ & 0,02 \\
$\mathrm{C}(64)-\mathrm{H}(64 \mathrm{C})$ & 1,828 & $-18,302$ & 0,02 \\
$\mathrm{C}(65)-\mathrm{H}(65 \mathrm{~A})$ & 1,824 & $-18,098$ & 0,01 \\
$\mathrm{C}(65)-\mathrm{H}(65 B)$ & 1,819 & $-17,848$ & 0,02 \\
$\mathrm{C}(65)-\mathrm{H}(65 \mathrm{C})$ & 1,833 & $-18,300$ & 0,02 \\
$\mathrm{C}(66)-\mathrm{C}(67)$ & 1,627 & $-9,385$ & 0,01 \\
$\mathrm{C}(66)-\mathrm{H}(66 \mathrm{~A})$ & 1,849 & $-18,420$ & 0,03 \\
$\mathrm{C}(66)-\mathrm{H}(66 \mathrm{~B})$ & 1,859 & $-18,617$ & 0,01 \\
$\mathrm{C}(67)-\mathrm{H}(67 \mathrm{~A})$ & 1,830 & $-18,217$ & 0,03 \\
$\mathrm{C}(67)-\mathrm{H}(67 \mathrm{~B})$ & 1,830 & $-18,280$ & 0,02 \\
$\mathrm{C}(67)-\mathrm{H}(67 \mathrm{C})$ & 1,827 & $-18,245$ & 0,02 \\
$\mathrm{C}(68)-\mathrm{H}(68 \mathrm{~A})$ & 1,969 & $-22,515$ & 0,02 \\
$\mathrm{C}(68)-\mathrm{H}(68 \mathrm{~B})$ & 1,950 & $-21,643$ & 0,04 \\
$\mathrm{C}(69)-\mathrm{C}(70)$ & 1,822 & $-14,369$ & 0,02 \\
$\mathrm{C}(69)-\mathrm{H}(69 \mathrm{~A})$ & 1,885 & $-21,061$ & 0,05 \\
$\mathrm{C}(69)-\mathrm{H}(69 B)$ & 1,889 & $-21,369$ & 0,05 \\
$\mathrm{C}(70)-\mathrm{H}(70 A)$ & 1,887 & $-21,218$ & 0,05 \\
$\mathrm{C}(70)-\mathrm{H}(70 B)$ & 1,896 & $-21,421$ & 0,04 \\
$\mathrm{C}(71)-\mathrm{H}(71 \mathrm{~A})$ & 1,873 & $-21,034$ & 0,05 \\
$\mathrm{C}(71)-\mathrm{H}(71 \mathrm{~B})$ & 1,887 & $-21,193$ & 0,05 \\
$\mathrm{C}(71)-\mathrm{H}(71 \mathrm{C})$ & 1,847 & $-20,035$ & 0,06 \\
\hline & & & \\
\hline
\end{tabular}


A. 1.4 Atomare Volumina und Ladungen aller Atome der Verfeinerungen von Roxithromycin

\begin{tabular}{|c|ccc|c|r|r|}
\hline & \multicolumn{3}{|c|}{ Ladung } & \multicolumn{3}{c|}{ V $_{\text {tot }}$} \\
\hline Atom & IV & EMV & GMV & IV & EMV & GMV \\
\hline O(14) & $-0,913$ & $-0,885$ & $-0,899$ & 12,8 & 12,9 & 12,9 \\
$\mathrm{O}(21)$ & $-1,042$ & $-0,914$ & $-0,919$ & 17,1 & 17,2 & 17,3 \\
$\mathrm{O}(22)$ & $-0,914$ & $-0,891$ & $-0,888$ & 13,9 & 14,4 & 14,5 \\
$\mathrm{O}(23)$ & $-0,899$ & $-0,872$ & $-0,885$ & 14,4 & 14,9 & 15,0 \\
$\mathrm{O}(24)$ & $-0,910$ & $-0,856$ & $-0,856$ & 15,1 & 15,4 & 15,4 \\
\hline
\end{tabular}




\begin{tabular}{|l|rrr|r|r|r|}
\hline & \multicolumn{3}{|c|}{ Ladung $\mathbf{Q}$} & \multicolumn{3}{|c|}{$\mathbf{V}_{\text {tot }}$} \\
\hline Atom & \multicolumn{1}{|c}{ IV } & EMV & GMV & \multicolumn{1}{|c|}{ IV } & EMV & GMV \\
\hline O(25) & $-0,676$ & $-0,585$ & $-0,592$ & 12,9 & 12,9 & 13,0 \\
O(26) & $-0,886$ & $-0,814$ & $-0,876$ & 12,7 & 12,7 & 13,0 \\
O(27) & $-0,846$ & $-0,761$ & $-0,761$ & 14,1 & 13,8 & 14,2 \\
O(5) & $-0,880$ & $-0,843$ & $-0,850$ & 11,5 & 11,6 & 11,6 \\
O(58) & $-0,851$ & $-0,812$ & $-0,820$ & 13,1 & 13,3 & 13,2 \\
O(59) & $-0,895$ & $-0,960$ & $-0,982$ & 17,4 & 18,7 & 18,7 \\
O(3) & $-0,856$ & $-0,822$ & $-0,829$ & 12,5 & 12,4 & 12,4 \\
O(38) & $-0,854$ & $-0,821$ & $-0,829$ & 14,1 & 14,6 & 14,5 \\
O(39) & $-0,918$ & $-0,889$ & $-0,891$ & 15,6 & 15,2 & 15,2 \\
O(40) & $-0,843$ & $-0,827$ & $-0,851$ & 12,8 & 12,7 & 12,8 \\
O(80) & $-1,019$ & $-0,958$ & $-0,889$ & 25,4 & 25,7 & 25,1 \\
N(2) & $-0,799$ & $-0,844$ & $-0,867$ & 9,7 & 9,5 & 9,6 \\
N(1) & $-0,483$ & $-0,421$ & $-0,423$ & 14,6 & 14,6 & 14,6 \\
C(1) & 1,274 & 1,379 & 1,401 & 5,3 & 5,0 & 5,0 \\
C(2) & $-0,024$ & 0,067 & 0,064 & 7,3 & 7,0 & 7,0 \\
C(3) & 0,293 & 0,330 & 0,336 & 6,2 & 6,1 & 6,1 \\
C(4) & $-0,035$ & 0,053 & 0,053 & 6,8 & 6,7 & 6,7 \\
C(5) & 0,275 & 0,312 & 0,319 & 6,3 & 6,2 & 6,2 \\
C(6) & 0,275 & 0,347 & 0,349 & 5,6 & 5,5 & 5,5 \\
C(7) & $-0,073$ & $-0,051$ & $-0,033$ & 7,7 & 7,8 & 7,8 \\
C(8) & $-0,024$ & 0,073 & 0,073 & 7,5 & 7,3 & 7,2 \\
C(9) & 0,425 & 0,404 & 0,424 & 7,2 & 7,3 & 7,3 \\
C(10) & $-0,015$ & 0,081 & 0,085 & 6,8 & 6,7 & 6,7 \\
C(11) & 0,285 & 0,318 & 0,333 & 6,2 & 6,1 & 6,1 \\
C(12) & 0,263 & 0,343 & 0,345 & 5,7 & 5,6 & 5,6 \\
C(13) & 0,273 & 0,311 & 0,318 & 6,2 & 6,0 & 6,0 \\
C(50) & 0,670 & 0,769 & 0,779 & 5,3 & 5,1 & 5,1 \\
C(51) & 0,269 & 0,299 & 0,310 & 6,4 & 6,3 & 6,3 \\
C(52) & 0,182 & 0,137 & 0,137 & 6,5 & 6,4 & 6,4 \\
C(53) & $-0,058$ & $-0,040$ & $-0,022$ & 8,3 & 8,5 & 8,4 \\
C(54) & 0,292 & 0,332 & 0,338 & 7,0 & 6,9 & 6,9 \\
C(55) & $-0,077$ & $-0,059$ & $-0,059$ & 11,8 & 11,8 & 11,8 \\
C(56) & 0,185 & 0,167 & 0,166 & 9,2 & 9,5 & 9,4 \\
C(57) & 0,177 & 0,155 & 0,152 & 9,4 & 9,5 & 9,5 \\
C(30) & 0,642 & 0,743 & 0,751 & 5,9 & 5,8 & 5,8 \\
\hline
\end{tabular}




\begin{tabular}{|l|rrr|r|r|r|}
\hline & \multicolumn{3}{|c|}{ Ladung $\mathbf{9}$} & \multicolumn{3}{|c|}{$\mathbf{V}_{\text {tot }}$} \\
\hline Atom & \multicolumn{1}{|c}{ IV } & EMV & GMV & IV & EMV & GMV \\
\hline C(31) & $-0,067$ & $-0,054$ & $-0,034$ & 8,4 & 8,6 & 8,5 \\
C(32) & 0,265 & 0,344 & 0,345 & 5,7 & 5,6 & 5,6 \\
C(33) & 0,276 & 0,318 & 0,329 & 6,2 & 6,1 & 6,1 \\
C(34) & 0,275 & 0,305 & 0,312 & 6,6 & 6,6 & 6,5 \\
C(35) & $-0,066$ & $-0,056$ & $-0,055$ & 10,9 & 11,3 & 11,3 \\
C(36) & $-0,067$ & $-0,047$ & $-0,047$ & 9,4 & 9,7 & 9,7 \\
C(37) & 0,261 & 0,114 & 0,281 & 10,3 & 11,7 & 10,6 \\
C(60) & $-0,075$ & $-0,063$ & $-0,064$ & 11,3 & 12,1 & 12,1 \\
C(61) & $-0,067$ & $-0,053$ & $-0,054$ & 9,7 & 10,0 & 9,9 \\
C(62) & $-0,066$ & $-0,051$ & $-0,053$ & 10,0 & 10,4 & 10,3 \\
C(63) & $-0,061$ & $-0,054$ & $-0,055$ & 9,9 & 10,2 & 10,2 \\
C(64) & $-0,063$ & $-0,043$ & $-0,043$ & 11,7 & 12,0 & 12,0 \\
C(65) & $-0,066$ & $-0,054$ & $-0,054$ & 10,4 & 11,0 & 10,9 \\
C(66) & $-0,051$ & $-0,029$ & $-0,012$ & 8,8 & 8,8 & 8,8 \\
C(67) & $-0,060$ & $-0,037$ & $-0,037$ & 11,4 & 11,6 & 11,6 \\
C(68) & 0,697 & 0,765 & 0,806 & 7,0 & 6,7 & 6,6 \\
C(69) & 0,280 & 0,211 & 0,293 & 8,6 & 9,5 & 8,8 \\
C(70) & 0,310 & 0,227 & 0,311 & 8,5 & 10,1 & 8,7 \\
C(71) & 0,250 & 0,007 & 0,259 & 11,8 & 15,5 & 12,6 \\
H(23) & 0,554 & 0,605 & 0,644 & 2,4 & 1,7 & 1,6 \\
H(24) & 0,545 & 0,551 & 0,558 & 2,5 & 2,3 & 2,4 \\
H(59) & 0,554 & 0,501 & 0,508 & 2,3 & 2,5 & 2,5 \\
H(39) & 0,547 & 0,480 & 0,466 & 1,8 & 1,9 & 1,9 \\
H(2) & 0,041 & 0,018 & 0,019 & 7,6 & 7,3 & 7,3 \\
H(3) & 0,050 & 0,009 & 0,005 & 6,0 & 6,2 & 6,0 \\
H(4) & 0,038 & 0,011 & 0,013 & 5,3 & 5,3 & 5,3 \\
H(5) & 0,047 & 0,009 & 0,005 & 6,0 & 6,0 & 6,0 \\
H(7A) & 0,047 & 0,015 & 0,013 & 6,7 & 6,7 & 6,7 \\
H(7B) & 0,053 & 0,016 & 0,014 & 7,0 & 6,9 & 6,9 \\
H(8) & 0,042 & 0,016 & 0,017 & 6,4 & 6,3 & 6,3 \\
H(10) & 0,039 & 0,013 & 0,014 & 6,1 & 6,2 & 6,3 \\
H(11) & 0,057 & 0,012 & 0,009 & 5,3 & 5,4 & 5,4 \\
H(13) & 0,053 & 0,010 & 0,007 & 6,5 & 6,7 & 6,6 \\
H(22) & 0,550 & 0,590 & 0,594 & 2,3 & 2,0 & 2,1 \\
H(50) & 0,088 & 0,071 & 0,088 & 4,9 & 4,8 & 4,8 \\
\hline
\end{tabular}




\begin{tabular}{|l|rrr|r|rr|}
\hline & \multicolumn{3}{|c|}{ Ladung $\mathbf{3}$} & \multicolumn{3}{c|}{$\mathbf{V}_{\text {tot }}$} \\
\hline Atom & IV & EMV & GMV & IV & EMV & GMV \\
\hline H(51) & 0,053 & 0,013 & 0,009 & 7,5 & 7,6 & 7,6 \\
H(52) & 0,018 & $-0,006$ & $-0,015$ & 8,8 & 9,1 & 9,0 \\
H(53A) & 0,050 & 0,018 & 0,016 & 7,5 & 7,3 & 7,3 \\
H(53B) & 0,050 & 0,013 & 0,010 & 7,1 & 7,2 & 7,0 \\
H(54) & 0,055 & 0,020 & 0,017 & 6,1 & 5,9 & 5,9 \\
H(55A) & 0,069 & 0,041 & 0,041 & 9,8 & 9,7 & 9,7 \\
H(55B) & 0,072 & 0,044 & 0,044 & 7,2 & 7,2 & 7,2 \\
H(55C) & 0,068 & 0,039 & 0,038 & 10,1 & 9,8 & 9,8 \\
H(56A) & 0,046 & 0,010 & 0,006 & 7,3 & 7,4 & 7,4 \\
H(56B) & 0,046 & 0,004 & 0,001 & 9,4 & 9,8 & 10,0 \\
H(56C) & 0,047 & 0,004 & 0,001 & 8,0 & 8,2 & 8,4 \\
H(57A) & 0,050 & 0,009 & 0,006 & 8,7 & 9,3 & 9,3 \\
H(57B) & 0,050 & 0,024 & 0,020 & 7,5 & 7,4 & 7,4 \\
H(57C) & 0,048 & 0,004 & 0,001 & 11,3 & 11,6 & 11,6 \\
H(30) & 0,089 & 0,081 & 0,097 & 5,9 & 5,7 & 5,6 \\
H(31A) & 0,049 & 0,013 & 0,012 & 11,2 & 11,4 & 11,4 \\
H(31B) & 0,052 & 0,017 & 0,015 & 6,1 & 6,0 & 6,0 \\
H(33) & 0,053 & 0,011 & 0,008 & 6,7 & 6,8 & 6,7 \\
H(34) & 0,051 & 0,013 & 0,010 & 4,9 & 4,9 & 4,9 \\
H(35A) & 0,070 & 0,042 & 0,041 & 8,1 & 7,8 & 7,9 \\
H(35B) & 0,067 & 0,038 & 0,037 & 9,7 & 9,6 & 9,6 \\
H(35C) & 0,073 & 0,046 & 0,046 & 8,8 & 8,7 & 8,7 \\
H(36A) & 0,068 & 0,041 & 0,040 & 7,5 & 7,2 & 7,2 \\
H(36B) & 0,070 & 0,038 & 0,037 & 7,5 & 7,5 & 7,6 \\
H(36C) & 0,067 & 0,042 & 0,041 & 6,8 & 6,6 & 6,7 \\
H(37A) & 0,078 & 0,081 & 0,078 & 7,9 & 7,4 & 7,7 \\
H(37B) & 0,075 & 0,074 & 0,075 & 9,3 & 8,7 & 9,1 \\
H(37C) & 0,075 & 0,075 & 0,075 & 8,9 & 8,2 & 8,6 \\
H(60A) & 0,066 & 0,036 & 0,035 & 9,1 & 9,1 & 9,1 \\
H(60B) & 0,068 & 0,040 & 0,039 & 8,3 & 8,2 & 8,2 \\
H(60C) & 0,072 & 0,045 & 0,045 & 10,2 & 10,2 & 10,2 \\
H(61A) & 0,062 & 0,038 & 0,037 & 7,9 & 7,7 & 7,7 \\
H(61B) & 0,069 & 0,042 & 0,041 & 6,4 & 6,4 & 6,4 \\
H(61C) & 0,071 & 0,041 & 0,040 & 8,7 & 8,4 & 8,4 \\
H(62A) & 0,066 & 0,040 & 0,038 & 9,8 & 9,8 & 9,8 \\
\hline
\end{tabular}




\begin{tabular}{|c|rrr|r|r|r|}
\hline & \multicolumn{3}{|c|}{ Ladung Q } & \multicolumn{3}{|c|}{ V $_{\text {tot }}$} \\
\hline Atom & IV & EMV & GMV & IV & EMV & GMV \\
\hline H(62B) & 0,067 & 0,039 & 0,038 & 6,4 & 6,3 & 6,3 \\
H(62C) & 0,067 & 0,038 & 0,037 & 8,6 & 8,6 & 8,6 \\
H(63A) & 0,066 & 0,034 & 0,034 & 7,0 & 6,9 & 6,9 \\
H(63B) & 0,065 & 0,040 & 0,039 & 6,6 & 6,6 & 6,6 \\
H(63C) & 0,065 & 0,035 & 0,035 & 8,0 & 7,9 & 8,0 \\
H(64A) & 0,067 & 0,040 & 0,039 & 10,5 & 10,2 & 10,2 \\
H(64B) & 0,069 & 0,040 & 0,039 & 7,2 & 7,1 & 7,2 \\
H(64C) & 0,068 & 0,036 & 0,035 & 10,0 & 9,9 & 10,0 \\
H(65A) & 0,066 & 0,040 & 0,040 & 9,8 & 9,5 & 9,6 \\
H(65B) & 0,068 & 0,042 & 0,040 & 7,4 & 7,4 & 7,4 \\
H(65C) & 0,066 & 0,038 & 0,037 & 10,0 & 9,8 & 9,8 \\
H(66A) & 0,050 & 0,012 & 0,010 & 7,6 & 7,8 & 7,7 \\
H(66B) & 0,054 & 0,013 & 0,011 & 7,0 & 6,8 & 6,8 \\
H(67A) & 0,067 & 0,039 & 0,038 & 9,7 & 9,4 & 9,4 \\
H(67B) & 0,067 & 0,037 & 0,036 & 9,0 & 8,8 & 8,9 \\
H(67C) & 0,069 & 0,040 & 0,039 & 6,1 & 6,0 & 6,0 \\
H(68A) & 0,098 & $-0,063$ & $-0,064$ & 8,3 & 8,4 & 8,2 \\
H(68B) & 0,100 & $-0,054$ & $-0,056$ & 5,8 & 6,1 & 6,1 \\
H(69A) & 0,058 & 0,097 & 0,060 & 10,3 & 9,7 & 10,4 \\
H(69B) & 0,050 & 0,094 & 0,055 & 9,1 & 8,3 & 8,9 \\
H(70A) & 0,061 & 0,116 & 0,067 & 7,3 & 6,4 & 7,0 \\
H(70B) & 0,054 & 0,111 & 0,062 & 7,6 & 6,9 & 7,3 \\
H(71A) & 0,072 & 0,226 & 0,073 & 8,3 & 6,1 & 7,9 \\
H(71B) & 0,077 & 0,229 & 0,079 & 7,9 & 6,2 & 7,7 \\
H(71C) & 0,088 & 0,225 & 0,091 & 8,2 & 6,2 & 7,8 \\
H(80A) & 0,522 & 0,462 & 0,469 & 2,5 & 2,5 & 2,4 \\
H(80B) & 0,518 & 0,517 & 0,494 & 3,1 & 2,4 & 2,5 \\
\hline \hline Summe & 0,067 & 0,030 & 0,070 & 1165,2 & 1163,2 & 1165,0 \\
\hline
\end{tabular}

\section{A.2 Invariome der Fluorochinolone}

Für die in der Invariom-Verfeinerung verwendetet Atomformfaktoren sind die Invariom-Namen und die zugehörigen Modellverbindung in englischer IUPACNomenklatur angegeben. Die zwölf Fluorochinolon-Strukturen sind alphabetisch geordnet. 
Tabelle A.1: Invariome der asphärischen Verfeinerung von Ciprofloxacin. $6 \mathrm{H}_{2} \mathrm{O}$.

\begin{tabular}{|c|c|c|}
\hline Atom & Invariom-Name & Modellverbindung \\
\hline $\mathrm{F}(15)$ & $\mathrm{F} @ 6 \mathrm{c}$ & 1-fluorobenzene \\
\hline $\mathrm{O}(12)$ & O1.5c[1.5o@6c] & benzoic acid anion \\
\hline $\mathrm{O}(13)$ & O1.5c[1.5o@6c] & benzoic acid anion \\
\hline $\mathrm{O}(14)$ & $0 @ 6 c$ & pyran-4-one ( $\gamma$-pyrone) \\
\hline $\mathrm{O}(2)$ & Olh1h & water \\
\hline $\mathrm{O}(3)$ & Olh1h & water \\
\hline $\mathrm{O}(4)$ & Olh1h & water \\
\hline $\mathrm{O}(5)$ & Olh1h & water \\
\hline $\mathrm{O}(6)$ & Olh1h & water \\
\hline $\mathrm{O}(7)$ & Olh1h & water \\
\hline $\mathrm{N}(1)$ & 6-N\#66c[\#66c\#6c]\#6c[\#6c1h]@3c & 1-cyclopropylquinolin-4-one \\
\hline $\mathrm{N}(20)$ & $\mathrm{N} @ 6 \mathrm{c} 1 \mathrm{c} 1 \mathrm{c}$ & $N, N$-dimethylbenzenamine \\
\hline $\mathrm{N}(23)$ & N1clclhlh & dimethylammonium \\
\hline $\mathrm{C}(16)$ & 3-C\#3c[\#3c1h1h]\#3c[\#3c1h1h]@6n1h & 1-cyclopropylpyridine-2-one \\
\hline $\mathrm{C}(18)$ & 3-C\#3c[\#3c@6n1h]\#3c[\#3c1h 1h]1h1h & 1-cyclopropylpyridine-2-one \\
\hline $\mathrm{C}(17)$ & 3-C\#3c[\#3c@6n1h]\#3c[\#3c1h1h]1h1h & 1-cyclopropylpyridine-2-one \\
\hline $\mathrm{C}(9)$ & 66-C\#6n[\#6c@3c]\#66c[\#6c\#6c]\#6c[\#6c1h] & 1-cyclopropylquinolin-2-one \\
\hline $\mathrm{C}(2)$ & 6-C\#6n[\#66c@3c]\#6c[\#6c1c]1h & 1-cyclopropyl-3-methylquinolin-4-one \\
\hline $\mathrm{C}(3)$ & 6-C\#6c[2o\#66c]\#6c[\#6n1h]1c & 3-methylquinolin-4-one \\
\hline $\mathrm{C}(11)$ & C1.5o1.5o@6c & benzoic acid anion \\
\hline $\mathrm{C}(4)$ & 6-C2o\#66c[\#66c\#6c]\#6c[\#6c1c] & 3-methyl-chromen-4-one \\
\hline $\mathrm{C}(10)$ & 66-C\#66c[\#6n\#6c]\#6c[2o\#6c]\#6c[\#6c1h] & quinolin-4-one \\
\hline $\mathrm{C}(5)$ & 6-C\#66c[\#66c\#6c]\#6c[\#6c1f]1h & 2-fluoronaphthalen \\
\hline $\mathrm{C}(6)$ & 6-C\#6c[\#6c1n]\#6c[\#66c1h]1f & 3-fluoronaphthalen-2-amine \\
\hline $\mathrm{C}(7)$ & 6-C\#6c[\#6c1f]\#6c[\#66c1h] $1 \mathrm{n}$ & 3-fluoronaphthalen-2-amine \\
\hline $\mathrm{C}(21)$ & Clnlclhlh & ethylamine \\
\hline $\mathrm{C}(22)$ & Clnlclhlh & ethylamine \\
\hline $\mathrm{C}(24)$ & Clnlclhlh & ethylamine \\
\hline $\mathrm{C}(25)$ & Clnlclhlh & ethylamine \\
\hline $\mathrm{C}(8)$ & 6-C\#66c[\#6n\#66c]\#6c[\#6c1n]1h & 7-aminoquinolin-2-one \\
\hline $\mathrm{H}(231)$ & $\operatorname{Hln}[1 \mathrm{c} 1 \mathrm{c} 1 \mathrm{~h}]$ & dimethylammonium \\
\hline $\mathrm{H}(232)$ & Hln[lclc1h] & dimethylammonium \\
\hline $\mathrm{H}(16)$ & $\mathrm{H} @ 3 \mathrm{c}$ & cyclopropane \\
\hline $\mathrm{H}(181)$ & $\mathrm{H} @ 3 \mathrm{c}$ & cyclopropane \\
\hline $\mathrm{H}(182)$ & $\mathrm{H} @ 3 c$ & cyclopropane \\
\hline $\mathrm{H}(171)$ & $\mathrm{H} @ 3 c$ & cyclopropane \\
\hline $\mathrm{H}(172)$ & $\mathrm{H} @ 3 \mathrm{c}$ & cyclopropane \\
\hline $\mathrm{H}(2)$ & H@6c & benzene \\
\hline $\mathrm{H}(5)$ & $\mathrm{H} @ 6 c$ & benzene \\
\hline $\mathrm{H}(211)$ & $\mathrm{H} 1 \mathrm{c}[\ln 1 \mathrm{c} 1 \mathrm{~h}]$ & ethylamine \\
\hline
\end{tabular}




\begin{tabular}{|c|c|c|}
\hline Atom & Invariom-Name & Modellverbindung \\
\hline $\mathrm{H}(212)$ & $\mathrm{Hlc}[\ln 1 \mathrm{clh}]$ & ethylamine \\
\hline $\mathrm{H}(221)$ & $\mathrm{Hlc}[\mathrm{lnlclh}]$ & ethylamine \\
\hline $\mathrm{H}(222)$ & $\mathrm{H} 1 \mathrm{c}[\mathrm{ln} 1 \mathrm{clh}]$ & ethylamine \\
\hline $\mathrm{H}(241)$ & $\mathrm{H} l \mathrm{c}[\mathrm{ln} l \mathrm{clh}]$ & ethylamine \\
\hline $\mathrm{H}(242)$ & $\mathrm{H} 1 \mathrm{c}[\mathrm{ln} 1 \mathrm{clh}]$ & ethylamine \\
\hline $\mathrm{H}(251)$ & $\mathrm{Hlc}[\mathrm{lnlclh}]$ & ethylamine \\
\hline $\mathrm{H}(252)$ & $\mathrm{H} 1 \mathrm{c}[\mathrm{ln} 1 \mathrm{clh}]$ & ethylamine \\
\hline $\mathrm{H}(8)$ & H@6c & benzene \\
\hline $\mathrm{H}(21)$ & $\mathrm{Hlo}[1 \mathrm{~h}]$ & water \\
\hline $\mathrm{H}(22)$ & $\mathrm{Hlo}[1 \mathrm{~h}]$ & water \\
\hline $\mathrm{H}(31)$ & $\mathrm{H} 1 \mathrm{o}[\mathrm{lh}]$ & water \\
\hline $\mathrm{H}(32)$ & $\mathrm{H} 1 \mathrm{o}[1 \mathrm{~h}]$ & water \\
\hline $\mathrm{H}(41)$ & $\mathrm{Hlo}[1 \mathrm{~h}]$ & water \\
\hline $\mathrm{H}(42)$ & $\mathrm{Hlo}[1 \mathrm{~h}]$ & water \\
\hline $\mathrm{H}(51)$ & $\mathrm{Hlo}[1 \mathrm{~h}]$ & water \\
\hline $\mathrm{H}(52)$ & $\mathrm{H} 1 \mathrm{o}[1 \mathrm{~h}]$ & water \\
\hline $\mathrm{H}(61)$ & $\mathrm{H} 1 \mathrm{o}[1 \mathrm{~h}]$ & water \\
\hline $\mathrm{H}(62)$ & $\mathrm{Hlo}[1 \mathrm{~h}]$ & water \\
\hline $\mathrm{H}(71)$ & $\mathrm{Hlo}[1 \mathrm{~h}]$ & water \\
\hline $\mathrm{H}(72)$ & $\mathrm{H} 1 \mathrm{o}[\mathrm{lh}]$ & water \\
\hline
\end{tabular}

Tabelle A.2: Invariome der asphärischen Verfeinerung von Ciprofloxacin $1,4 \mathrm{H}_{2} \mathrm{O} \cdot \mathrm{HCl}$.

\begin{tabular}{|c|c|c|}
\hline Atom & Invariom-Name & Modellverbindung \\
\hline $\mathrm{Cl}-(1)$ & $\mathrm{Cl}$ & chloride \\
\hline $\mathrm{F}(15)$ & F@6c & 1-fluorobenzene \\
\hline $\mathrm{O}(12)$ & $\mathrm{O} 2 \mathrm{c}$ & formaldehyde \\
\hline $\mathrm{O}(13)$ & Olclh & methanol \\
\hline $\mathrm{O}(14)$ & o@6c & pyran-4-one ( $\gamma$-pyrone) \\
\hline $\mathrm{O}(2)$ & Olhlh & water \\
\hline $\mathrm{O}(31)$ & Olhlh & water \\
\hline $\mathrm{N}(1)$ & 6-N\#66c[\#66c\#6c]\#6c[\#6c1h]@3c & 1-cyclopropylquinolin-4-one \\
\hline $\mathrm{N}(20)$ & N@6c1c1c & $N, N$-dimethylbenzenamine \\
\hline $\mathrm{N}(23)$ & Nlclclh1h & dimethylammonium \\
\hline$C(16)$ & 3-C\#3c[\#3c1h1h]\#3c[\#3c1h1h]@6n1h & 1-cyclopropylpyridine-2-one \\
\hline$C(18)$ & 3-C\#3c[\#3c@6n1h]\#3c[\#3c1h1h]1h1h & 1-cyclopropylpyridine-2-one \\
\hline $\mathrm{C}(17)$ & 3-C\#3c[\#3c@6n1h]\#3c[\#3c1h1h]1h1h & 1-cyclopropylpyridine-2-one \\
\hline $\mathrm{C}(9)$ & 66-C\#6n[\#6c@3c]\#66c[\#6c\#6c]\#6c[\#6c1h] & 1-cyclopropylquinolin-2-one \\
\hline$C(2)$ & 6-C\#6n[\#66c@3c]\#6c[\#6c1c]1h & 1-cyclopropyl-3-methylquinolin-4-one \\
\hline $\mathrm{C}(3)$ & 6-C\#6c[2o\#66c]\#6c[\#6n1h]1c & 3-methylquinolin-4-one \\
\hline
\end{tabular}




\begin{tabular}{|c|c|c|}
\hline Atom & Invariom-Name & Modellverbindung \\
\hline $\mathrm{C}(11)$ & C2o1o@6c & benzoic acid \\
\hline $\mathrm{C}(4)$ & 6-C2o\#66c[\#66c\#6c]\#6c[\#6c1c] & 3-methyl-chromen-4-one \\
\hline $\mathrm{C}(10)$ & 66-C\#66c[\#6n\#6c]\#6c[2o\#6c]\#6c[\#6c1h] & quinolin-4-one \\
\hline $\mathrm{C}(5)$ & 6-C\#66c[\#66c\#6c]\#6c[\#6c1f] $1 \mathrm{~h}$ & 2-fluoronaphthalen \\
\hline $\mathrm{C}(6)$ & 6-C\#6c[\#6c1n]\#6c[\#66c1h]1f & 3-fluoronaphthalen-2-amine \\
\hline $\mathrm{C}(7)$ & 6-C\#6c[\#6c1f] $\# 6 c[\# 66 \mathrm{c} 1 \mathrm{~h}] \ln$ & 3-fluoronaphthalen-2-amine \\
\hline $\mathrm{C}(21)$ & Clnlclhlh & ethylamine \\
\hline $\mathrm{C}(22)$ & Clnlclhlh & ethylamine \\
\hline $\mathrm{C}(24)$ & Clnlclhlh & ethylamine \\
\hline $\mathrm{C}(25)$ & Clnlclhlh & ethylamine \\
\hline $\mathrm{C}(8)$ & 6-C\#66c[\#6n\#66c]\#6c[\#6c1n]1h & 7-aminoquinolin-2-one \\
\hline $\mathrm{H}(13)$ & $\mathrm{H} 1 \mathrm{o}[1 \mathrm{c}]$ & methanol \\
\hline $\mathrm{H}(231)$ & $\mathrm{H} \ln [1 \mathrm{clc} 1 \mathrm{~h}]$ & dimethylammonium \\
\hline $\mathrm{H}(232)$ & $\mathrm{H} \ln [1 \mathrm{c} 1 \mathrm{c} 1 \mathrm{~h}]$ & dimethylammonium \\
\hline $\mathrm{H}(16)$ & $\mathrm{H} @ 3 \mathrm{c}$ & cyclopropane \\
\hline $\mathrm{H}(181)$ & $\mathrm{H} @ 3 \mathrm{c}$ & cyclopropane \\
\hline $\mathrm{H}(182)$ & $\mathrm{H} @ 3 c$ & cyclopropane \\
\hline $\mathrm{H}(171)$ & $\mathrm{H} @ 3 \mathrm{c}$ & cyclopropane \\
\hline $\mathrm{H}(172)$ & $\mathrm{H} @ 3 \mathrm{c}$ & cyclopropane \\
\hline $\mathrm{H}(2)$ & $\mathrm{H} @ 6 \mathrm{c}$ & benzene \\
\hline $\mathrm{H}(5)$ & $\mathrm{H} @ 6 c$ & benzene \\
\hline $\mathrm{H}(211)$ & $\mathrm{H} 1 \mathrm{c}[\ln 1 \mathrm{c} 1 \mathrm{~h}]$ & ethylamine \\
\hline $\mathrm{H}(212)$ & $\mathrm{H} 1 \mathrm{c}[\ln 1 \mathrm{c} 1 \mathrm{~h}]$ & ethylamine \\
\hline $\mathrm{H}(221)$ & $\mathrm{H} 1 \mathrm{c}[\ln 1 \mathrm{c} 1 \mathrm{~h}]$ & ethylamine \\
\hline $\mathrm{H}(222)$ & $\mathrm{H} 1 \mathrm{c}[\ln 1 \mathrm{c} 1 \mathrm{~h}]$ & ethylamine \\
\hline $\mathrm{H}(241)$ & $\mathrm{H} 1 \mathrm{c}[\ln 1 \mathrm{c} 1 \mathrm{~h}]$ & ethylamine \\
\hline $\mathrm{H}(242)$ & $\mathrm{H} 1 \mathrm{c}[\ln 1 \mathrm{c} 1 \mathrm{~h}]$ & ethylamine \\
\hline $\mathrm{H}(251)$ & $\mathrm{H} 1 \mathrm{c}[\ln 1 \mathrm{c} 1 \mathrm{~h}]$ & ethylamine \\
\hline $\mathrm{H}(252)$ & $\mathrm{H} 1 \mathrm{c}[\ln 1 \mathrm{c} 1 \mathrm{~h}]$ & ethylamine \\
\hline $\mathrm{H}(8)$ & $\mathrm{H} @ 6 \mathrm{c}$ & benzene \\
\hline $\mathrm{H}(21)$ & Hlo[1h] & water \\
\hline $\mathrm{H}(22)$ & $\mathrm{H} 1 \mathrm{o}[1 \mathrm{~h}]$ & water \\
\hline $\mathrm{H}(31)$ & H1o[1h] & water \\
\hline $\mathrm{H}(32)$ & H1o[1h] & water \\
\hline
\end{tabular}

Tabelle A.3: Invariome der asphärischen Verfeinerung von Enoxacin· 1,7 $\mathrm{H}_{2} \mathrm{O}$.

\begin{tabular}{lll} 
Atom & Invariom-Name & Modellverbindung \\
\hline $\mathrm{F}(15)$ & $\mathrm{F} @ 6 \mathrm{c}$ & 1-fluorobenzene \\
$\mathrm{F}(65)$ & $\mathrm{F} @ 6 \mathrm{c}$ & 1-fluorobenzene \\
$\mathrm{O}(12)$ & $\mathrm{O} 1.5 \mathrm{c}[1.5 \mathrm{o} @ 6 \mathrm{c}]$ & benzoic acid anion \\
\hline
\end{tabular}




\begin{tabular}{|c|c|c|}
\hline Atom & Invariom-Name & Modellverbindung \\
\hline $\mathrm{O}(13)$ & O1.5c[1.5o@6c] & benzoic acid anion \\
\hline $\mathrm{O}(14)$ & O@6c & pyran-4-one ( $\gamma$-pyrone) \\
\hline $\mathrm{O}(62)$ & O1.5c[1.5o@6c] & benzoic acid anion \\
\hline $\mathrm{O}(63)$ & O1.5c[1.5o@6c] & benzoic acid anion \\
\hline $\mathrm{O}(64)$ & O@6c & pyran-4-one ( $\gamma$-pyrone) \\
\hline $\mathrm{O}(1)$ & Olh1h & water \\
\hline $\mathrm{O}(2)$ & Olh1h & water \\
\hline $\mathrm{O}(3)$ & Olh1h & water \\
\hline $\mathrm{O}(4)$ & Olhlh & water \\
\hline $\mathrm{O}(5)$ & Olhlh & water \\
\hline $\mathrm{N}(1)$ & 6-N\#66c[\#6n\#66c]\#6c[\#6c1h]1c & 1-methyl-1,8-naphthyridin-4-one \\
\hline $\mathrm{N}(8)$ & 6-N\#66c[\#6n\#66c]\#6c[\#6c1n] & 1,8-naphthyridin-2-amine \\
\hline $\mathrm{N}(20)$ & $\mathrm{N} @ 6 c 1 \mathrm{c} 1 \mathrm{c}$ & $N, N$-dimethylbenzenamine \\
\hline $\mathrm{N}(23)$ & N1clclhlh & dimethylammonium \\
\hline $\mathrm{N}(51)$ & 6-N\#66c[\#6n\#66c]\#6c[\#6c1h]1c & 1-methyl-1,8-naphthyridin-4-one \\
\hline $\mathrm{N}(58)$ & 6-N\#66c[\#6n\#66c]\#6c[\#6c1n] & 1,8-naphthyridin-2-amine \\
\hline $\mathrm{N}(70)$ & $\mathrm{N} @ 6 c 1 \mathrm{c} 1 \mathrm{c}$ & $N, N$-dimethylbenzenamine \\
\hline $\mathrm{N}(73)$ & Nlclclhlh & dimethylammonium \\
\hline $\mathrm{C}(2)$ & $6-\mathrm{C} \# 6 \mathrm{n}[\# 66 \mathrm{c} 1 \mathrm{c}] \# 6 \mathrm{c}[\# 6 \mathrm{c} 1 \mathrm{c}] 1 \mathrm{~h}$ & 1,3-dimethylquinolin-4-one \\
\hline $\mathrm{C}(3)$ & 6-C\#6c[2o\#66c]\#6c[\#6n 1h] $1 \mathrm{c}$ & 3-methylquinolin-4-one \\
\hline $\mathrm{C}(4)$ & 6-C2o\#66c[\#66c\#6c]\#6c[\#6c1c] & 3-methyl-chromen-4-one \\
\hline $\mathrm{C}(5)$ & 6-C\#66c[\#66c\#6c]\#6c[\#6c1f]1h & 2-fluoronaphthalen \\
\hline $\mathrm{C}(6)$ & 6-C\#6c[\#6n $1 \mathrm{n}] \# 6 c[\# 66 \mathrm{c} 1 \mathrm{~h}] 1 \mathrm{f}$ & 3-fluoroquinolin-2-amine \\
\hline $\mathrm{C}(7)$ & 6-C\#6n[\#66c]\#6c[\#6c1f] $1 \mathrm{n}$ & 3-fluoroquinolin-2-amine \\
\hline $\mathrm{C}(9)$ & 66-C\#6n[\#6c1c]\#6n[\#6c]\#66c[\#6c\#6c] & 1-methyl-1,8-naphthyridin-4-one \\
\hline $\mathrm{C}(10)$ & 66-C\#66c[\#6n\#6n]\#6c[2o\#6c]\#6c[\#6c1h] & 1,8-naphthyridin-4-one \\
\hline $\mathrm{C}(11)$ & C1.5o1.5o@6c & benzoic acid anion \\
\hline $\mathrm{C}(16)$ & C@6n1c1h1h & 1-ethylpylpyridine-2-one \\
\hline $\mathrm{C}(17)$ & Clclhlhlh & ethane \\
\hline $\mathrm{C}(21)$ & Clnlclhlh & ethylamine \\
\hline $\mathrm{C}(22)$ & Clnlclhlh & ethylamine \\
\hline $\mathrm{C}(24)$ & Clnlclhlh & ethylamine \\
\hline $\mathrm{C}(25)$ & Clnlclhlh & ethylamine \\
\hline $\mathrm{C}(52)$ & 6-C\#6n[\#66c1c]\#6c[\#6c1c]1h & 1,3-dimethylquinolin-4-one \\
\hline $\mathrm{C}(53)$ & 6-C\#6c[2o\#66c]\#6c[\#6n $1 \mathrm{~h}] 1 \mathrm{c}$ & 3-methylquinolin-4-one \\
\hline $\mathrm{C}(54)$ & 6-C2o\#66c[\#66c\#6c]\#6c[\#6c1c] & 3-methyl-chromen-4-one \\
\hline $\mathrm{C}(55)$ & 6-C\#66c[\#66c\#6c]\#6c[\#6c1f]1h & 2-fluoronaphthalen \\
\hline $\mathrm{C}(56)$ & 6-C\#6c[\#6n 1n]\#6c[\#66c1h]1f & 3-fluoroquinolin-2-amine \\
\hline $\mathrm{C}(57)$ & 6-C\#6n[\#66c]\#6c[\#6c1f] $1 \mathrm{n}$ & 3-fluoroquinolin-2-amine \\
\hline $\mathrm{C}(59)$ & 66-C\#6n[\#6c1c]\#6n[\#6c]\#66c[\#6c\#6c] & 1-methyl-1,8-naphthyridin-4-one \\
\hline $\mathrm{C}(60)$ & 66-C\#66c[\#6n\#6n]\#6c[2o\#6c]\#6c[\#6c1h] & 1,8-naphthyridin-4-one \\
\hline $\mathrm{C}(61)$ & C1.5o1.5o@6c & benzoic acid anion \\
\hline $\mathrm{C}(66)$ & C@6n1c1h1h & 1-ethylpylpyridine-2-one \\
\hline
\end{tabular}




\begin{tabular}{|c|c|c|}
\hline Atom & Invariom-Name & Modellverbindung \\
\hline $\mathrm{C}(67)$ & Clclhlh1h & ethane \\
\hline $\mathrm{C}(71)$ & Clnlclhlh & ethylamine \\
\hline $\mathrm{C}(72)$ & Clnlclhlh & ethylamine \\
\hline $\mathrm{C}(74)$ & Clnlclhlh & ethylamine \\
\hline$C(75)$ & Clnlclhlh & ethylamine \\
\hline $\mathrm{H}(2)$ & $\mathrm{H} @ 6 c$ & benzene \\
\hline $\mathrm{H}(5)$ & $\mathrm{H} @ 6 c$ & benzene \\
\hline $\mathrm{H}(161)$ & H1c[@6n1c1h] & 1-ethylpylpyridine-2-one \\
\hline $\mathrm{H}(612)$ & H1c[@6n1c1h] & 1-ethylpylpyridine-2-one \\
\hline $\mathrm{H}(171)$ & H1c[1clh1h] & ethane \\
\hline $\mathrm{H}(172)$ & $\mathrm{H} 1 \mathrm{c}[1 \mathrm{c} 1 \mathrm{~h} 1 \mathrm{~h}]$ & ethane \\
\hline $\mathrm{H}(173)$ & $\mathrm{H} 1 \mathrm{c}[1 \mathrm{c} 1 \mathrm{~h} 1 \mathrm{~h}]$ & ethane \\
\hline $\mathrm{H}(221)$ & $\mathrm{H} 1 \mathrm{c}[\ln 1 \mathrm{c} 1 \mathrm{~h}]$ & ethylamine \\
\hline $\mathrm{H}(222)$ & $\mathrm{H} 1 \mathrm{c}[\ln 1 \mathrm{c} 1 \mathrm{~h}]$ & ethylamine \\
\hline $\mathrm{H}(232)$ & $\mathrm{H} \ln [1 \mathrm{c} 1 \mathrm{c} 1 \mathrm{~h}]$ & dimethylammonium \\
\hline $\mathrm{H}(231)$ & $\mathrm{H} \ln [1 \mathrm{c} 1 \mathrm{c} 1 \mathrm{~h}]$ & dimethylammonium \\
\hline $\mathrm{H}(241)$ & $\mathrm{H} 1 \mathrm{c}[\ln 1 \mathrm{c} 1 \mathrm{~h}]$ & ethylamine \\
\hline $\mathrm{H}(242)$ & $\mathrm{H} 1 \mathrm{c}[\ln 1 \mathrm{c} 1 \mathrm{~h}]$ & ethylamine \\
\hline $\mathrm{H}(251)$ & $\mathrm{H} 1 \mathrm{c}[\ln 1 \mathrm{c} 1 \mathrm{~h}]$ & ethylamine \\
\hline $\mathrm{H}(252)$ & $\mathrm{H} 1 \mathrm{c}[\ln 1 \mathrm{c} 1 \mathrm{~h}]$ & ethylamine \\
\hline $\mathrm{H}(211)$ & $\mathrm{H} 1 \mathrm{c}[\ln 1 \mathrm{c} 1 \mathrm{~h}]$ & ethylamine \\
\hline $\mathrm{H}(212)$ & $\mathrm{H} 1 \mathrm{c}[\ln 1 \mathrm{c} 1 \mathrm{~h}]$ & ethylamine \\
\hline $\mathrm{H}(52)$ & $\mathrm{H} @ 6 \mathrm{c}$ & benzene \\
\hline $\mathrm{H}(55)$ & $\mathrm{H} @ 6 c$ & benzene \\
\hline $\mathrm{H}(661)$ & H1c[@6n1c1h] & 1-ethylpylpyridine-2-one \\
\hline $\mathrm{H}(662)$ & H1c[@6n1c1h] & 1-ethylpylpyridine-2-one \\
\hline $\mathrm{H}(671)$ & $\mathrm{H} 1 \mathrm{c}[1 \mathrm{c} 1 \mathrm{~h} 1 \mathrm{~h}]$ & ethane \\
\hline $\mathrm{H}(672)$ & $\mathrm{H} 1 \mathrm{c}[1 \mathrm{c} 1 \mathrm{~h} 1 \mathrm{~h}]$ & ethane \\
\hline $\mathrm{H}(673)$ & $\mathrm{H} 1 \mathrm{c}[1 \mathrm{c} 1 \mathrm{~h} 1 \mathrm{~h}]$ & ethane \\
\hline $\mathrm{H}(711)$ & $\mathrm{H} 1 \mathrm{c}[\ln 1 \mathrm{c} 1 \mathrm{~h}]$ & ethylamine \\
\hline $\mathrm{H}(712)$ & $\mathrm{H} 1 \mathrm{c}[\ln 1 \mathrm{c} 1 \mathrm{~h}]$ & ethylamine \\
\hline $\mathrm{H}(721)$ & $\mathrm{H} 1 \mathrm{c}[\ln 1 \mathrm{c} 1 \mathrm{~h}]$ & ethylamine \\
\hline $\mathrm{H}(722)$ & $\mathrm{H} 1 \mathrm{c}[\ln 1 \mathrm{c} 1 \mathrm{~h}]$ & ethylamine \\
\hline $\mathrm{H}(731)$ & $\mathrm{H} 1 \mathrm{n}[1 \mathrm{c} 1 \mathrm{c} 1 \mathrm{~h}]$ & dimethylammonium \\
\hline $\mathrm{H}(732)$ & $\mathrm{H} \ln [1 \mathrm{c} 1 \mathrm{c} 1 \mathrm{~h}]$ & dimethylammonium \\
\hline $\mathrm{H}(741)$ & $\mathrm{H} 1 \mathrm{c}[\ln 1 \mathrm{c} 1 \mathrm{~h}]$ & ethylamine \\
\hline $\mathrm{H}(742)$ & $\mathrm{H} 1 \mathrm{c}[\ln 1 \mathrm{c} 1 \mathrm{~h}]$ & ethylamine \\
\hline $\mathrm{H}(751)$ & $\mathrm{H} 1 \mathrm{c}[\ln 1 \mathrm{c} 1 \mathrm{~h}]$ & ethylamine \\
\hline $\mathrm{H}(752)$ & $\mathrm{H} 1 \mathrm{c}[\ln 1 \mathrm{c} 1 \mathrm{~h}]$ & ethylamine \\
\hline $\mathrm{H}(11)$ & $\mathrm{H} 1 \mathrm{o}[1 \mathrm{~h}]$ & water \\
\hline $\mathrm{H}(12)$ & $\mathrm{H} 1 \mathrm{o}[1 \mathrm{~h}]$ & water \\
\hline $\mathrm{H}(21)$ & $\mathrm{H} 1 \mathrm{o}[\mathrm{lh}]$ & water \\
\hline $\mathrm{H}(22)$ & $\mathrm{H} 1 \mathrm{o}[1 \mathrm{~h}]$ & water \\
\hline
\end{tabular}




\begin{tabular}{lll} 
Atom & Invariom-Name & Modellverbindung \\
\hline $\mathrm{H}(31)$ & $\mathrm{H} 1 \mathrm{o}[1 \mathrm{~h}]$ & water \\
$\mathrm{H}(32)$ & $\mathrm{H} 1 \mathrm{o}[1 \mathrm{~h}]$ & water \\
$\mathrm{H}(41)$ & $\mathrm{H} 1 \mathrm{o}[1 \mathrm{~h}]$ & water \\
$\mathrm{H}(42)$ & $\mathrm{H} 1 \mathrm{o}[1 \mathrm{~h}]$ & water \\
$\mathrm{H}(51)$ & $\mathrm{H} 1 \mathrm{o}[1 \mathrm{~h}]$ & water \\
$\mathrm{H}(53)$ & $\mathrm{H} 1 \mathrm{o}[1 \mathrm{~h}]$ & water \\
\hline
\end{tabular}

Tabelle A.4: Invariome der asphärischen Verfeinerung von Enoxacin·3 $\mathrm{H}_{2} \mathrm{O}$.

\begin{tabular}{|c|c|c|}
\hline Atom & Invariom-Name & Modellverbindung \\
\hline $\mathrm{F}(15)$ & F@6c & 1-fluorobenzene \\
\hline $\mathrm{O}(14)$ & O@6c & pyran-4-one ( $\gamma$-pyrone) \\
\hline $\mathrm{O}(12)$ & O1.5c[1.5o@6c] & benzoic acid anion \\
\hline $\mathrm{O}(13)$ & O1.5c[1.5o@6c] & benzoic acid anion \\
\hline $\mathrm{O}(4)$ & Olhlh & water \\
\hline $\mathrm{O}(5)$ & Olhlh & water \\
\hline $\mathrm{O}(6)$ & Olhlh & water \\
\hline $\mathrm{N}(1)$ & 6-N\#66c[\#6n\#66c]\#6c[\#6c1h]1c & 1-methyl-1,8-naphthyridin-4-one \\
\hline $\mathrm{N}(8)$ & 6-N\#66c[\#6n\#66c]\#6c[\#6c1n] & 1,8-naphthyridin-2-amine \\
\hline $\mathrm{N}(20)$ & 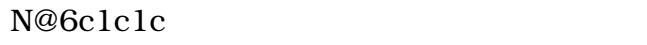 & $N, N$-dimethylbenzenamine \\
\hline $\mathrm{N}(23)$ & Nlclclhlh & dimethylammonium \\
\hline $\mathrm{C}(2)$ & 6-C\#6n[\#66c1c]\#6c[\#6c1c]1h & 1,3-dimethylquinolin-4-one \\
\hline $\mathrm{C}(3)$ & 6-C\#6c[2o\#66c]\#6c[\#6n1h]1c & 3-methylquinolin-4-one \\
\hline $\mathrm{C}(4)$ & 6-C2o\#66c[\#66c\#6c]\#6c[\#6c1c] & 3-methyl-chromen-4-one \\
\hline$C(10)$ & 66-C\#66c[\#6n\#6n]]\#c[2o\#6c]\#6c[\#6c1h] & 1,8-naphthyridin-4-one \\
\hline $\mathrm{C}(5)$ & 6-C\#66c[\#66c\#6c]\#6c[\#6c1f]1h & 2-fluoronaphthalen \\
\hline $\mathrm{C}(6)$ & 6-C\#6c[\#6n1n]\#6c[\#66c1h]1f & 3-fluoroquinolin-2-amine \\
\hline$C(7)$ & 6-C\#6n[\#66c]\#6c[\#6c1f] $1 \mathrm{n}$ & 3-fluoroquinolin-2-amine \\
\hline $\mathrm{C}(9)$ & 66-C\#6n[\#6c1c]\#6n[\#6c]\#66c[\#6c\#6c] & 1-methyl-1,8-naphthyridin-4-one \\
\hline$C(16)$ & C@6n1c1hlh & 1-ethylpylpyridine-2-one \\
\hline $\mathrm{C}(17)$ & Clclhlhlh & ethane \\
\hline$C(11)$ & C1.5o1.5o@6c & benzoic acid anion \\
\hline $\mathrm{C}(25)$ & Clnlclhlh & ethylamine \\
\hline $\mathrm{C}(24)$ & Clnlclhlh & ethylamine \\
\hline $\mathrm{C}(22)$ & Clnlclhlh & ethylamine \\
\hline $\mathrm{C}(21)$ & Clnlclhlh & ethylamine \\
\hline $\mathrm{H}(23 \mathrm{~A})$ & $\mathrm{H} \ln [1 \mathrm{clc} 1 \mathrm{~h}]$ & dimethylammonium \\
\hline $\mathrm{H}(23 \mathrm{~B})$ & $\mathrm{H} 1 \mathrm{n}[1 \mathrm{clc} 1 \mathrm{~h}]$ & dimethylammonium \\
\hline $\mathrm{H}(2)$ & H@6c & benzene \\
\hline
\end{tabular}




\begin{tabular}{|c|c|c|}
\hline Atom & Invariom-Name & Modellverbindung \\
\hline $\mathrm{H}(5)$ & H@6c & benzene \\
\hline $\mathrm{H}(16 \mathrm{~A})$ & H1c[@6n1c1h] & 1-ethylpylpyridine-2-one \\
\hline $\mathrm{H}(16 \mathrm{~B})$ & H1c[@6n1c1h] & 1-ethylpylpyridine-2-one \\
\hline $\mathrm{H}(17 \mathrm{~A})$ & $\mathrm{H} 1 \mathrm{c}[\mathrm{lc} \operatorname{lh} 1 \mathrm{~h}]$ & ethane \\
\hline $\mathrm{H}(17 \mathrm{~B})$ & $\mathrm{H} 1 \mathrm{c}[1 \mathrm{c} 1 \mathrm{~h} 1 \mathrm{~h}]$ & ethane \\
\hline $\mathrm{H}(17 \mathrm{C})$ & $\mathrm{H} 1 \mathrm{c}[1 \mathrm{c} 1 \mathrm{~h} 1 \mathrm{~h}]$ & ethane \\
\hline $\mathrm{H}(25 \mathrm{~A})$ & $\mathrm{H} 1 \mathrm{c}[\ln 1 \mathrm{c} 1 \mathrm{~h}]$ & ethylamine \\
\hline $\mathrm{H}(25 \mathrm{~B})$ & $\mathrm{H} 1 \mathrm{c}[\ln 1 \mathrm{c} 1 \mathrm{~h}]$ & ethylamine \\
\hline $\mathrm{H}(24 \mathrm{~A})$ & $\mathrm{H} 1 \mathrm{c}[\ln 1 \mathrm{c} 1 \mathrm{~h}]$ & ethylamine \\
\hline $\mathrm{H}(24 \mathrm{~B})$ & $\mathrm{H} 1 \mathrm{c}[\ln 1 \mathrm{c} 1 \mathrm{~h}]$ & ethylamine \\
\hline $\mathrm{H}(22 \mathrm{~A})$ & $\mathrm{H} 1 \mathrm{c}[\ln 1 \mathrm{c} 1 \mathrm{~h}]$ & ethylamine \\
\hline $\mathrm{H}(22 \mathrm{~B})$ & $\mathrm{H} 1 \mathrm{c}[\ln 1 \mathrm{c} 1 \mathrm{~h}]$ & ethylamine \\
\hline $\mathrm{H}(21 \mathrm{~A})$ & $\mathrm{H} 1 \mathrm{c}[\ln 1 \mathrm{c} 1 \mathrm{~h}]$ & ethylamine \\
\hline $\mathrm{H}(21 \mathrm{~B})$ & $\mathrm{H} 1 \mathrm{c}[\ln 1 \mathrm{clh}]$ & ethylamine \\
\hline $\mathrm{H}(041)$ & $\mathrm{H} 1 \mathrm{o}[1 \mathrm{~h}]$ & water \\
\hline $\mathrm{H}(042)$ & $\mathrm{H} 1 \mathrm{o}[\mathrm{lh}]$ & water \\
\hline $\mathrm{H}(051)$ & $\mathrm{H} 1 \mathrm{o}[1 \mathrm{~h}]$ & water \\
\hline $\mathrm{H}(052)$ & $\mathrm{H} 1 \mathrm{o}[\mathrm{lh}]$ & water \\
\hline $\mathrm{H}(061)$ & $\mathrm{H} 1 \mathrm{o}[\mathrm{lh}]$ & water \\
\hline $\mathrm{H}(062)$ & $\mathrm{H} 1 \mathrm{o}[\mathrm{lh}]$ & water \\
\hline
\end{tabular}

Tabelle A.5: Invariome der asphärischen Verfeinerung von Fleroxacin $\cdot \mathrm{H}_{2} \mathrm{O} \cdot \mathrm{HCl}$.

\begin{tabular}{|c|c|c|}
\hline Atom & Invariom-Name & Modellverbindung \\
\hline $\mathrm{Cl}-(1)$ & $\mathrm{Cl}$ & chloride \\
\hline$F(15)$ & $\mathrm{F} @ 6 \mathrm{c}$ & 1-fluorobenzene \\
\hline $\mathrm{F}(8)$ & $\mathrm{F} @ 6 \mathrm{c}$ & 1-fluorobenzene \\
\hline $\mathrm{F}(17)$ & F1c & fluoromethane \\
\hline $\mathrm{O}(13)$ & Olclh & methanol \\
\hline $\mathrm{O}(12)$ & $\mathrm{O} 2 \mathrm{c}$ & formaldehyde \\
\hline $\mathrm{O}(14)$ & O@6c & pyran-4-one ( $\gamma$-pyrone) \\
\hline $\mathrm{O}(2)$ & Olhlh & water \\
\hline $\mathrm{N}(1)$ & $6-\mathrm{N} \# 66 \mathrm{c}[\# 66 \mathrm{c} \# 6 \mathrm{c}] \# 6 \mathrm{c}[\# 6 \mathrm{c} 1 \mathrm{~h}] 1 \mathrm{c}$ & 1,3-dimethylquinolin-4-one \\
\hline $\mathrm{N}(20)$ & $\mathrm{N} @ 6 c 1 \mathrm{c} 1 \mathrm{c}$ & $N, N$-dimethylbenzenamine \\
\hline $\mathrm{N}(23)$ & $\mathrm{N} 1 \mathrm{clclc}$ lh & trimethylammonium \\
\hline $\mathrm{C}(11)$ & C2o1o@6c & benzoic acid \\
\hline $\mathrm{C}(3)$ & 6-C\#6c[2o\#66c]\#6c[\#6n $1 \mathrm{~h}] 1 \mathrm{c}$ & 3-methylquinolin-4-one \\
\hline $\mathrm{C}(4)$ & 6-C2o\#66c[\#66c\#6c]\#6c[\#6c1c] & 3-methyl-chromen-4-one \\
\hline $\mathrm{C}(10)$ & 66-C\#66c[\#6n\#6c]\#6c[2o\#6c]\#6c[\#6c1h] & quinolin-4-one \\
\hline $\mathrm{C}(9)$ & 66-C\#6n[\#6c1c]\#66c[\#6c\#6c]\#6c[\#6c1f] & 8-fluoro-1-methylquinolin-2-one \\
\hline
\end{tabular}




\begin{tabular}{|c|c|c|}
\hline Atom & Invariom-Name & Modellverbindung \\
\hline $\mathrm{C}(2)$ & 6-C\#6n[\#66c1c]\#6c[\#6c1c]1h & 1,3-dimethylquinolin-4-one \\
\hline $\mathrm{C}(16)$ & C@6n1c1h1h & 1-ethylpylpyridine-2-one \\
\hline $\mathrm{C}(17)$ & Clflclhlh & fluoroethane \\
\hline $\mathrm{C}(8)$ & 6-C\#66c[\#6n\#66c]\#6c[\#6cln]1f & 7-amino-8-fluoroquinolin-2-one \\
\hline $\mathrm{C}(7)$ & 6-C\#6c[\#66c1f]\#6c[\#6c1f] 1n & 1,3-difluoronaphthalen-2-amine \\
\hline $\mathrm{C}(6)$ & 6-C\#6c[\#6c1n]\#6c[\#66c1h]1f & 3-fluoronaphthalen-2-amine \\
\hline $\mathrm{C}(5)$ & 6-C\#66c[\#66c\#6c]\#6c[\#6c1f] $1 \mathrm{~h}$ & 2-fluoronaphthalen \\
\hline $\mathrm{C}(25)$ & Clnlclhlh & ethylamine \\
\hline $\mathrm{C}(24)$ & Clnlclhlh & ethylamine \\
\hline $\mathrm{C}(19)$ & Clnlclhlh & ethylamine \\
\hline $\mathrm{C}(21)$ & Clnlclhlh & ethylamine \\
\hline $\mathrm{C}(26)$ & Cln1h1h1h & methylamine \\
\hline $\mathrm{H}(13)$ & $\mathrm{H} 1 \mathrm{o}[1 \mathrm{c}]$ & methanol \\
\hline $\mathrm{H}(23)$ & $\mathrm{H} \ln [1 \mathrm{c} 1 \mathrm{c} 1 \mathrm{c}]$ & trimethylammonium \\
\hline $\mathrm{H}(2)$ & H@6c & benzene \\
\hline $\mathrm{H}(161)$ & H1c[@6n1c1h] & 1-ethylpylpyridine-2-one \\
\hline $\mathrm{H}(162)$ & H1c[@6n1c1h] & 1-ethylpylpyridine-2-one \\
\hline $\mathrm{H}(171)$ & $\mathrm{H} 1 \mathrm{c}[1 \mathrm{f} 1 \mathrm{c} 1 \mathrm{~h}]$ & fluoroethane \\
\hline $\mathrm{H}(172)$ & $\mathrm{H} 1 \mathrm{c}[1 \mathrm{flclh}]$ & fluoroethane \\
\hline $\mathrm{H}(5)$ & $\mathrm{H} @ 6 c$ & benzene \\
\hline $\mathrm{H}(251)$ & $\mathrm{H} 1 \mathrm{c}[\ln 1 \mathrm{c} 1 \mathrm{~h}]$ & ethylamine \\
\hline $\mathrm{H}(252)$ & $\mathrm{H} 1 \mathrm{c}[\ln 1 \mathrm{c} 1 \mathrm{~h}]$ & ethylamine \\
\hline $\mathrm{H}(241)$ & $\mathrm{H} 1 \mathrm{c}[\ln 1 \mathrm{c} 1 \mathrm{~h}]$ & ethylamine \\
\hline $\mathrm{H}(242)$ & $\mathrm{H} 1 \mathrm{c}[\ln 1 \mathrm{c} 1 \mathrm{~h}]$ & ethylamine \\
\hline $\mathrm{H}(191)$ & $\mathrm{H} 1 \mathrm{c}[\ln 1 \mathrm{c} 1 \mathrm{~h}]$ & ethylamine \\
\hline $\mathrm{H}(192)$ & $\mathrm{H} 1 \mathrm{c}[\ln 1 \mathrm{c} 1 \mathrm{~h}]$ & ethylamine \\
\hline $\mathrm{H}(211)$ & $\mathrm{H} 1 \mathrm{c}[\ln 1 \mathrm{c} 1 \mathrm{~h}]$ & ethylamine \\
\hline $\mathrm{H}(212)$ & $\mathrm{H} 1 \mathrm{c}[\ln 1 \mathrm{c} 1 \mathrm{~h}]$ & ethylamine \\
\hline $\mathrm{H}(261)$ & $\mathrm{H} 1 \mathrm{c}[\ln 1 \mathrm{~h} 1 \mathrm{~h}]$ & methylamine \\
\hline $\mathrm{H}(262)$ & $\mathrm{H} 1 \mathrm{c}[\ln 1 \mathrm{~h} 1 \mathrm{~h}]$ & methylamine \\
\hline $\mathrm{H}(263)$ & $\mathrm{H} 1 \mathrm{c}[\ln 1 \mathrm{~h} 1 \mathrm{~h}]$ & methylamine \\
\hline $\mathrm{H}(21)$ & $\mathrm{H} 1 \mathrm{o}[\mathrm{lh}]$ & water \\
\hline $\mathrm{H}(22)$ & $\mathrm{H} 1 \mathrm{o}[1 \mathrm{~h}]$ & water \\
\hline
\end{tabular}

Tabelle A.6: Invariome der asphärischen Verfeinerung von Lomefloxacin.4 $\mathrm{H}_{2} \mathrm{O} \cdot 0,95 \mathrm{Cl}^{-}$.

\begin{tabular}{lll} 
Atom & Invariom-Name & Modellverbindung \\
\hline $\mathrm{Cl}-(1)$ & $\mathrm{Cl}$ & chloride \\
$\mathrm{F}(17)$ & $\mathrm{F} @ 6 \mathrm{c}$ & 1-fluorobenzene \\
$\mathrm{F}(18)$ & $\mathrm{F} @ 6 \mathrm{c}$ & 1-fluorobenzene \\
$\mathrm{O}(1)$ & $\mathrm{O} 1 \mathrm{~h} 1 \mathrm{~h}$ & water \\
\hline
\end{tabular}




\begin{tabular}{|c|c|c|}
\hline Atom & Invariom-Name & Modellverbindung \\
\hline $\mathrm{O}(2)$ & Olhlh & water \\
\hline $\mathrm{O}(12)$ & $\mathrm{O} 2 \mathrm{c}$ & formaldehyde \\
\hline $\mathrm{O}(13)$ & Olclh & methanol \\
\hline $\mathrm{O}(14)$ & O@6c & pyran-4-one ( $\gamma$-pyrone) \\
\hline $\mathrm{N}(1)$ & $6-N \# 66 c[\# 66 c \# 6 c] \# 6 c[\# 6 c 1 h] 1 c$ & 1,3-dimethylquinolin-4-one \\
\hline $\mathrm{N}(20)$ & $\mathrm{N} @ 6 \mathrm{c} 1 \mathrm{c} 1 \mathrm{c}$ & $N, N$-dimethylbenzenamine \\
\hline $\mathrm{N}(23 \mathrm{~A})$ & N1clclh1h & dimethylammonium \\
\hline $\mathrm{N}(23 \mathrm{~B})$ & N1clclh1h & dimethylammonium \\
\hline $\mathrm{C}(2)$ & 6-C\#6n[\#66c1c]\#6c[\#6c1c]1h & 1,3-dimethylquinolin-4-one \\
\hline $\mathrm{C}(3)$ & $6-\mathrm{C} \# 6 c[2 o \# 66 \mathrm{c}] \# 6 \mathrm{c}[\# 6 \mathrm{n} 1 \mathrm{~h}] 1 \mathrm{c}$ & 3-methylquinolin-4-one \\
\hline $\mathrm{C}(5)$ & 6-C\#66c[\#66c\#6c]\#6c[\#6clf] $1 \mathrm{~h}$ & 2-fluoronaphthalen \\
\hline $\mathrm{C}(4)$ & 6-C2o\#66c[\#66c\#6c]\#6c[\#6c1c] & 3-methyl-chromen-4-one \\
\hline $\mathrm{C}(6)$ & 6-C\#6c[\#6cln]\#6c[\#66c1h]1f & 3-fluoronaphthalen-2-amine \\
\hline $\mathrm{C}(7)$ & 6-C\#6c[\#66c1f] \#6c[\#6c1f] $1 \mathrm{n}$ & 1,3-difluoronaphthalen-2-amine \\
\hline $\mathrm{C}(9)$ & 66-C\#6n[\#6c1c]\#66c[\#6c\#6c]\#6c[\#6c1f] & 8-fluoro-1-methylquinolin-2-one \\
\hline $\mathrm{C}(8)$ & 6-C\#66c[\#6n\#66c]\#6c[\#6c1n]1f & 7-amino-8-fluoroquinolin-2-one \\
\hline$C(10)$ & 66-C\#66c[\#6n\#6c]\#6c[2o\#6c]\#6c[\#6c1h] & quinolin-4-one \\
\hline $\mathrm{C}(11)$ & C2o1o@6c & benzoic acid \\
\hline $\mathrm{C}(15)$ & C@6n1c1h1h & 1-ethylpylpyridine-2-one \\
\hline$C(16)$ & C1clhlh1h & ethane \\
\hline $\mathrm{C}(21 \mathrm{~A})$ & Clnlclhlh & ethylamine \\
\hline $\mathrm{C}(22 \mathrm{~A})$ & Cln $1 \mathrm{clc} 1 \mathrm{~h}$ & 2-aminopropane \\
\hline $\mathrm{C}(24 \mathrm{~A})$ & Clnlclhlh & ethylamine \\
\hline $\mathrm{C}(25 \mathrm{~A})$ & Clnlclhlh & ethylamine \\
\hline $\mathrm{C}(26 \mathrm{~A})$ & Clclhlhlh & ethane \\
\hline$C(21 B)$ & Cln $1 \mathrm{c} 1 \mathrm{~h} 1 \mathrm{~h}$ & ethylamine \\
\hline $\mathrm{C}(22 \mathrm{~B})$ & Cln $1 \mathrm{c} 1 \mathrm{c} 1 \mathrm{~h}$ & 2-aminopropane \\
\hline $\mathrm{C}(24 \mathrm{~B})$ & Clnlclhlh & ethylamine \\
\hline $\mathrm{C}(25 \mathrm{~B})$ & Clnlclhlh & ethylamine \\
\hline $\mathrm{C}(26 \mathrm{~B})$ & Clclhlhlh & ethane \\
\hline $\mathrm{H}(1 \mathrm{~A})$ & $\mathrm{H} 1 \mathrm{o}[1 \mathrm{~h}]$ & water \\
\hline $\mathrm{H}(1 \mathrm{~B})$ & $\mathrm{H} 1 \mathrm{o}[1 \mathrm{~h}]$ & water \\
\hline $\mathrm{H}(2 \mathrm{~A})$ & $\mathrm{H} 1 \mathrm{o}[1 \mathrm{~h}]$ & water \\
\hline $\mathrm{H}(2 \mathrm{~B})$ & $\mathrm{H} 1 \mathrm{o}[\mathrm{lh}]$ & water \\
\hline $\mathrm{H}(2)$ & $\mathrm{H} @ 6 c$ & benzene \\
\hline $\mathrm{H}(5)$ & $\mathrm{H} @ 6 c$ & benzene \\
\hline $\mathrm{H}(13)$ & $\mathrm{H} 1 \mathrm{o}[1 \mathrm{c}]$ & methanol \\
\hline $\mathrm{H}(15 \mathrm{~A})$ & H1c[@6n1c1h] & 1-ethylpylpyridine-2-one \\
\hline$H(15 B)$ & H1c[@6n1c1h] & 1-ethylpylpyridine-2-one \\
\hline $\mathrm{H}(16 \mathrm{~A})$ & $\mathrm{H} 1 \mathrm{c}[\mathrm{lc} 1 \mathrm{~h} 1 \mathrm{~h}]$ & ethane \\
\hline $\mathrm{H}(16 \mathrm{~B})$ & $\mathrm{H} 1 \mathrm{c}[1 \mathrm{c} 1 \mathrm{~h} 1 \mathrm{~h}]$ & ethane \\
\hline $\mathrm{H}(16 \mathrm{C})$ & $\mathrm{H} 1 \mathrm{c}[1 \mathrm{c} 1 \mathrm{~h} 1 \mathrm{~h}]$ & ethane \\
\hline $\mathrm{H}(23 \mathrm{~A})$ & $\mathrm{H} \ln [1 \mathrm{clc} 1 \mathrm{~h}]$ & dimethylammonium \\
\hline
\end{tabular}




\begin{tabular}{|c|c|c|}
\hline Atom & Invariom-Name & Modellverbindung \\
\hline $\mathrm{H}(23 \mathrm{~B})$ & H1n[1clclh] & dimethylammonium \\
\hline $\mathrm{H}(21 \mathrm{~A})$ & Hlc[lnlclh] & ethylamine \\
\hline $\mathrm{H}(21 \mathrm{~B})$ & $\mathrm{H} 1 \mathrm{c}[\mathrm{ln} 1 \mathrm{c} 1 \mathrm{~h}]$ & ethylamine \\
\hline $\mathrm{H}(22 \mathrm{~A})$ & $\mathrm{Hlc}[\operatorname{lnlclc}]$ & 2-aminopropane \\
\hline $\mathrm{H}(24 \mathrm{C})$ & $\mathrm{H} 1 \mathrm{c}[\mathrm{ln} 1 \mathrm{c} 1 \mathrm{~h}]$ & ethylamine \\
\hline $\mathrm{H}(24 \mathrm{D})$ & Hlc[lnlclh] & ethylamine \\
\hline $\mathrm{H}(25 \mathrm{~A})$ & $\mathrm{H} 1 \mathrm{c}[\mathrm{ln} 1 \mathrm{c} 1 \mathrm{~h}]$ & ethylamine \\
\hline $\mathrm{H}(25 \mathrm{C})$ & $\mathrm{H} 1 \mathrm{c}[\mathrm{ln} 1 \mathrm{c} 1 \mathrm{~h}]$ & ethylamine \\
\hline $\mathrm{H}(26 \mathrm{~A})$ & Hlc[lclhlh] & ethane \\
\hline $\mathrm{H}(26 \mathrm{~B})$ & H1c[1clhlh] & ethane \\
\hline $\mathrm{H}(26 \mathrm{C})$ & $\mathrm{H} 1 \mathrm{c}[1 \mathrm{c} 1 \mathrm{~h} 1 \mathrm{~h}]$ & ethane \\
\hline $\mathrm{H}(23 \mathrm{C})$ & $\mathrm{H} \ln [\mathrm{lclclh}]$ & dimethylammonium \\
\hline $\mathrm{H}(23 \mathrm{D})$ & $\mathrm{H} \ln [\mathrm{lclclh}]$ & dimethylammonium \\
\hline $\mathrm{H}(21 \mathrm{C})$ & Hlc[lnlclh] & ethylamine \\
\hline $\mathrm{H}(21 \mathrm{D})$ & Hlc[lnlclh] & ethylamine \\
\hline $\mathrm{H}(22 \mathrm{~B})$ & $\mathrm{H} 1 \mathrm{c}[\mathrm{ln} 1 \mathrm{clc}]$ & 2-aminopropane \\
\hline $\mathrm{H}(24 \mathrm{E})$ & $\mathrm{H} 1 \mathrm{c}[\mathrm{ln} 1 \mathrm{c} 1 \mathrm{~h}]$ & ethylamine \\
\hline $\mathrm{H}(24 \mathrm{~F})$ & Hlc[lnlclh] & ethylamine \\
\hline $\mathrm{H}(25 \mathrm{D})$ & $\mathrm{H} 1 \mathrm{c}[\mathrm{ln} 1 \mathrm{c} 1 \mathrm{~h}]$ & ethylamine \\
\hline $\mathrm{H}(25 \mathrm{E})$ & $\mathrm{H} 1 \mathrm{c}[\mathrm{ln} 1 \mathrm{clh}]$ & ethylamine \\
\hline $\mathrm{H}(26 \mathrm{D})$ & $\mathrm{H} 1 \mathrm{c}[\mathrm{lc} \operatorname{lh} 1 \mathrm{~h}]$ & ethane \\
\hline $\mathrm{H}(26 \mathrm{E})$ & $\mathrm{H} 1 \mathrm{c}[\mathrm{lc} 1 \mathrm{~h} 1 \mathrm{~h}]$ & ethane \\
\hline $\mathrm{H}(26 \mathrm{~F})$ & Hlc[lclhlh] & ethane \\
\hline
\end{tabular}

Tabelle A.7: Invariome der asphärischen Verfeinerung von Moxifloxacin $0,5 \mathrm{H}_{2} \mathrm{O} \cdot 0,5 \mathrm{CH}_{3} \mathrm{OH} \cdot \mathrm{HCl}$.

\begin{tabular}{|c|c|c|}
\hline Atom & Invariom-Name & Modellverbindung \\
\hline $\mathrm{Cl}-(1)$ & $\mathrm{Cl}$ & chloride \\
\hline $\mathrm{Cl}-(2)$ & $\mathrm{Cl}$ & chloride \\
\hline $\mathrm{F}(15)$ & F@6c & 1-fluorobenzene \\
\hline $\mathrm{F}(65)$ & F@6c & 1-fluorobenzene \\
\hline $\mathrm{O}(12)$ & Olclh & methanol \\
\hline $\mathrm{O}(13)$ & $\mathrm{O} 2 \mathrm{c}$ & formaldehyde \\
\hline $\mathrm{O}(14)$ & O@6c & pyran-4-one ( $\gamma$-pyrone) \\
\hline $\mathrm{O}(19)$ & $0 @ 6 \mathrm{clc}$ & anisole \\
\hline $\mathrm{O}(63)$ & Olclh & methanol \\
\hline $\mathrm{O}(62)$ & $\mathrm{O} 2 \mathrm{c}$ & formaldehyde \\
\hline O(64) & o@6c & pyran-4-one ( $\gamma$-pyrone) \\
\hline O(69) & $0 @ 6 \mathrm{clc}$ & anisole \\
\hline $\mathrm{O}(4)$ & Olclh & methanol \\
\hline $\mathrm{O}(42)$ & Olhlh & water \\
\hline
\end{tabular}




\begin{tabular}{|c|c|c|}
\hline Atom & Invariom-Name & Modellverbindung \\
\hline $\mathrm{N}(1)$ & 6-N\#66c[\#66c\#6c]\#6c[\#6c1h]@3c & 1-cyclopropylquinolin-4-one \\
\hline $\mathrm{N}(20)$ & $\mathrm{N} @ 6 \mathrm{c} 1 \mathrm{c} 1 \mathrm{c}$ & $N, N$-dimethylbenzenamine \\
\hline $\mathrm{N}(23)$ & N1clclh1h & dimethylammonium \\
\hline $\mathrm{N}(51)$ & 6-N\#66c[\#66c\#6c]\#6c[\#6c1h]@3c & 1-cyclopropylquinolin-4-one \\
\hline $\mathrm{N}(70)$ & $\mathrm{N} @ 6 c 1 \mathrm{clc}$ & $N, N$-dimethylbenzenamine \\
\hline $\mathrm{N}(73)$ & N1clclhlh & dimethylammonium \\
\hline $\mathrm{C}(2)$ & 6-C\#6n[\#66c@3c]\#6c[\#6c1c]1h & 1-cyclopropyl-3-methylquinolin-4-one \\
\hline $\mathrm{C}(3)$ & 6-C\#6c[2o\#66c]\#6c[\#6n 1h] lc & 3-methylquinolin-4-one \\
\hline $\mathrm{C}(4)$ & 6-C2o\#66c[\#66c\#6c]\#6c[\#6c1c] & 3-methyl-chromen-4-one \\
\hline $\mathrm{C}(10)$ & 66-C\#66c[\#6n\#6c]\#6c[2o\#6c]\#6c[\#6clh] & quinolin-4-one \\
\hline $\mathrm{C}(5)$ & 6-C\#66c[\#66c\#6c]\#6c[\#6c1f] $1 \mathrm{~h}$ & 2-fluoronaphthalen \\
\hline $\mathrm{C}(6)$ & 6-C\#6c[\#6c1n]\#6c[\#66c1h]1f & 3-fluoronaphthalen-2-amine \\
\hline $\mathrm{C}(7)$ & $6-\mathrm{C} \# 6 \mathrm{c}[\# 6 \mathrm{c} 1 \mathrm{f}] \# 6 \mathrm{c}[\# 66 \mathrm{c} 1 \mathrm{o}] \ln$ & 2-amino-3-fluoronaphthalen-1-ol \\
\hline $\mathrm{C}(8)$ & 6-C\#66c[\#6n\#66c]\#6c[\#6c1n]1o & 7-amino-8-hydroxyquinolin-2-one \\
\hline $\mathrm{C}(9)$ & 66-C\#6n[\#6c@3c]\#66c[\#6c\#6c]\#6c[\#6c1o] & 1-cyclopropyl-8-hydroxyquinolin-2(1H)-one \\
\hline $\mathrm{C}(11)$ & C2o1o@6c & benzoic acid \\
\hline $\mathrm{C}(16)$ & 3-C\#3c[\#3c1h 1h]\#3c[\#3c1h1h]@6n1h & 1-cyclopropylpyridine-2-one \\
\hline $\mathrm{C}(17)$ & 3-C\#3c[\#3c@6n1h]\#3c[\#3c1h1h]1h1h & 1-cyclopropylpyridine-2-one \\
\hline $\mathrm{C}(18)$ & 3-C\#3c[\#3c@6n1h]\#3c[\#3c1h 1h]1h $1 \mathrm{~h}$ & 1-cyclopropylpyridine-2-one \\
\hline $\mathrm{C}(19)$ & Clolhlhlh & methanol \\
\hline $\mathrm{C}(21)$ & Clnlclhlh & ethylamine \\
\hline $\mathrm{C}(22)$ & Clnlclclh & 2-aminopropane \\
\hline $\mathrm{C}(24)$ & Clnlclhlh & ethylamine \\
\hline $\mathrm{C}(25)$ & Clclclhlh & propane \\
\hline $\mathrm{C}(26)$ & Clclclhlh & propane \\
\hline $\mathrm{C}(27)$ & Clclclclh & isobutane \\
\hline $\mathrm{C}(28)$ & Clnlclhlh & ethylamine \\
\hline $\mathrm{C}(52)$ & 6-C\#6n[\#66c@3c]\#6c[\#6c1c]1h & 1-cyclopropyl-3-methylquinolin-4-one \\
\hline $\mathrm{C}(53)$ & 6-C\#6c[2o\#66c]\#6c[\#6n 1h]1c & 3-methylquinolin-4-one \\
\hline $\mathrm{C}(54)$ & 6-C2o\#66c[\#66c\#6c]\#6c[\#6c1c] & 3-methyl-chromen-4-one \\
\hline $\mathrm{C}(60)$ & 66-C\#66c[\#6n\#6c]\#6c[2o\#6c]\#6c[\#6c1h] & quinolin-4-one \\
\hline $\mathrm{C}(55)$ & 6-C\#66c[\#66c\#6c]\#6c[\#6c1f] $1 \mathrm{~h}$ & 2-fluoronaphthalen \\
\hline $\mathrm{C}(56)$ & 6-C\#6c[\#6c1n]\#6c[\#66c1h]1f & 3-fluoronaphthalen-2-amine \\
\hline $\mathrm{C}(57)$ & $6-\mathrm{C} \# 6 \mathrm{c}[\# 6 \mathrm{c} 1 \mathrm{f}] \# 6 \mathrm{c}[\# 66 \mathrm{c} 1 \mathrm{lo}] \mathrm{ln}$ & 2-amino-3-fluoronaphthalen-1-ol \\
\hline $\mathrm{C}(58)$ & 6-C\#66c[\#6n\#66c]\#6c[\#6c1n]1o & 7-amino-8-hydroxyquinolin-2-one \\
\hline $\mathrm{C}(59)$ & 66-C\#6n[\#6c@3c]\#66c[\#6c\#6c]\#6c[\#6c1o] & 1-cyclopropyl-8-hydroxy-1,8a-dihydroquinolin-2(3H)-one \\
\hline $\mathrm{C}(61)$ & C2o1o@6c & benzoic acid \\
\hline $\mathrm{C}(66)$ & 3-C\#3c[\#3c1h1h]\#3c[\#3c1h1h]@6n1h & 1-cyclopropylpyridine-2-one \\
\hline $\mathrm{C}(67)$ & 3-C\#3c[\#3c@6n1h]\#3c[\#3c1h1h]1h1h & 1-cyclopropylpyridine-2-one \\
\hline $\mathrm{C}(68)$ & 3-C\#3c[\#3c@6n1h]\#3c[\#3c1h1h]1h1h & 1-cyclopropylpyridine-2-one \\
\hline $\mathrm{C}(69)$ & Clolhlhlh & methanol \\
\hline $\mathrm{C}(71)$ & Clnlclhlh & ethylamine \\
\hline $\mathrm{C}(72)$ & Clnlclc1h & 2-aminopropane \\
\hline
\end{tabular}




\begin{tabular}{|c|c|c|}
\hline Atom & Invariom-Name & Modellverbindung \\
\hline $\mathrm{C}(74)$ & Clnlclh1h & ethylamine \\
\hline$C(75)$ & Clclclhlh & propane \\
\hline$C(76)$ & Clclclhlh & propane \\
\hline $\mathrm{C}(77)$ & Clclclclh & isobutane \\
\hline $\mathrm{C}(78)$ & Clnlclhlh & ethylamine \\
\hline $\mathrm{C}(41)$ & Clolhlhlh & methanol \\
\hline $\mathrm{H}(12)$ & $\mathrm{H} 1 \mathrm{o}[1 \mathrm{c}]$ & methanol \\
\hline $\mathrm{H}(23 \mathrm{~A})$ & $\mathrm{H} 1 \mathrm{n}[1 \mathrm{clclh}]$ & dimethylammonium \\
\hline $\mathrm{H}(23 \mathrm{~B})$ & $\mathrm{H} \ln [1 \mathrm{clclh}]$ & dimethylammonium \\
\hline $\mathrm{H}(2)$ & $\mathrm{H} @ 6 \mathrm{c}$ & benzene \\
\hline $\mathrm{H}(5)$ & $\mathrm{H} @ 6 \mathrm{c}$ & benzene \\
\hline $\mathrm{H}(16)$ & $\mathrm{H} @ 3 c$ & cyclopropane \\
\hline $\mathrm{H}(17 \mathrm{~A})$ & $\mathrm{H} @ 3 \mathrm{c}$ & cyclopropane \\
\hline $\mathrm{H}(17 \mathrm{~B})$ & $\mathrm{H} @ 3 \mathrm{c}$ & cyclopropane \\
\hline $\mathrm{H}(18 \mathrm{~A})$ & $\mathrm{H} @ 3 c$ & cyclopropane \\
\hline $\mathrm{H}(18 \mathrm{~B})$ & $\mathrm{H} @ 3 \mathrm{c}$ & cyclopropane \\
\hline $\mathrm{H}(19 \mathrm{~A})$ & $\mathrm{H} 1 \mathrm{c}[1 \mathrm{olh} 1 \mathrm{~h}]$ & methanol \\
\hline $\mathrm{H}(19 \mathrm{~B})$ & H1c[1olh $1 \mathrm{~h}]$ & methanol \\
\hline $\mathrm{H}(19 \mathrm{C})$ & Hlc [1olhlh] & methanol \\
\hline $\mathrm{H}(21 \mathrm{~A})$ & $\mathrm{H} 1 \mathrm{c}[\mathrm{ln} 1 \mathrm{c} 1 \mathrm{~h}]$ & ethylamine \\
\hline $\mathrm{H}(21 \mathrm{~B})$ & $\mathrm{H} 1 \mathrm{c}[\mathrm{ln} 1 \mathrm{clh}]$ & ethylamine \\
\hline $\mathrm{H}(22 \mathrm{~A})$ & $\mathrm{H} 1 \mathrm{c}[\ln 1 \mathrm{clc}]$ & 2-aminopropane \\
\hline $\mathrm{H}(24 \mathrm{~A})$ & $\mathrm{H} 1 \mathrm{c}[\ln 1 \mathrm{clh}]$ & ethylamine \\
\hline $\mathrm{H}(24 \mathrm{~B})$ & $\mathrm{H} 1 \mathrm{c}[\mathrm{ln} 1 \mathrm{clh}]$ & ethylamine \\
\hline $\mathrm{H}(25 \mathrm{~A})$ & $\mathrm{H} 1 \mathrm{c}[1 \mathrm{clclh}]$ & propane \\
\hline $\mathrm{H}(25 \mathrm{~B})$ & $\mathrm{H} l \mathrm{c}[\mathrm{lclclh}]$ & propane \\
\hline $\mathrm{H}(26 \mathrm{~A})$ & $\mathrm{H} 1 \mathrm{c}[\mathrm{lclclh}]$ & propane \\
\hline $\mathrm{H}(26 \mathrm{~B})$ & $\mathrm{H} 1 \mathrm{c}[\mathrm{lclclh}]$ & propane \\
\hline $\mathrm{H}(27)$ & $\mathrm{H} 1 \mathrm{c}[\mathrm{lclclc}]$ & isobutane \\
\hline $\mathrm{H}(28 \mathrm{~A})$ & $\mathrm{H} 1 \mathrm{c}[\mathrm{ln} 1 \mathrm{clh}]$ & ethylamine \\
\hline $\mathrm{H}(28 \mathrm{~B})$ & $\mathrm{H} 1 \mathrm{c}[\ln 1 \mathrm{c} 1 \mathrm{~h}]$ & ethylamine \\
\hline $\mathrm{H}(63)$ & $\mathrm{H} 1 \mathrm{o}[1 \mathrm{c}]$ & methanol \\
\hline $\mathrm{H}(73 \mathrm{~A})$ & $\mathrm{H} \ln [\mathrm{lclclh}]$ & dimethylammonium \\
\hline $\mathrm{H}(73 \mathrm{~B})$ & $\mathrm{H} \ln [1 \mathrm{clclh}]$ & dimethylammonium \\
\hline $\mathrm{H}(52)$ & H@6c & benzene \\
\hline $\mathrm{H}(55)$ & $\mathrm{H} @ 6 \mathrm{c}$ & benzene \\
\hline $\mathrm{H}(66)$ & $\mathrm{H} @ 3 c$ & cyclopropane \\
\hline $\mathrm{H}(67 \mathrm{~A})$ & $\mathrm{H} @ 3 c$ & cyclopropane \\
\hline $\mathrm{H}(67 \mathrm{~B})$ & $\mathrm{H} @ 3 \mathrm{c}$ & cyclopropane \\
\hline $\mathrm{H}(68 \mathrm{~A})$ & $\mathrm{H} @ 3 \mathrm{c}$ & cyclopropane \\
\hline $\mathrm{H}(68 \mathrm{~B})$ & $\mathrm{H} @ 3 \mathrm{c}$ & cyclopropane \\
\hline $\mathrm{H}(69 \mathrm{~A})$ & H1c[1olhlh] & methanol \\
\hline $\mathrm{H}(69 \mathrm{~B})$ & H1c[1olhlh] & methanol \\
\hline
\end{tabular}




\begin{tabular}{|c|c|c|}
\hline Atom & Invariom-Name & Modellverbindung \\
\hline $\mathrm{H}(69 \mathrm{C})$ & H1c[1olh1h] & methanol \\
\hline $\mathrm{H}(71 \mathrm{~A})$ & $\mathrm{H} 1 \mathrm{c}[\ln 1 \mathrm{c} 1 \mathrm{~h}]$ & ethylamine \\
\hline $\mathrm{H}(71 \mathrm{~B})$ & $\mathrm{H} 1 \mathrm{c}[\ln 1 \mathrm{c} 1 \mathrm{~h}]$ & ethylamine \\
\hline $\mathrm{H}(72)$ & $\mathrm{H} 1 \mathrm{c}[\ln 1 \mathrm{c} 1 \mathrm{c}]$ & 2-aminopropane \\
\hline $\mathrm{H}(74 \mathrm{~A})$ & $\mathrm{H} 1 \mathrm{c}[\ln 1 \mathrm{c} 1 \mathrm{~h}]$ & ethylamine \\
\hline $\mathrm{H}(74 \mathrm{~B})$ & $\mathrm{H} 1 \mathrm{c}[\ln 1 \mathrm{c} 1 \mathrm{~h}]$ & ethylamine \\
\hline $\mathrm{H}(75 \mathrm{~A})$ & $\mathrm{H} 1 \mathrm{c}[1 \mathrm{c} 1 \mathrm{c} 1 \mathrm{~h}]$ & propane \\
\hline $\mathrm{H}(75 \mathrm{~B})$ & $\mathrm{H} 1 \mathrm{c}[1 \mathrm{c} 1 \mathrm{c} 1 \mathrm{~h}]$ & propane \\
\hline $\mathrm{H}(76 \mathrm{~A})$ & $\mathrm{H} 1 \mathrm{c}[1 \mathrm{c} 1 \mathrm{c} 1 \mathrm{~h}]$ & propane \\
\hline $\mathrm{H}(76 \mathrm{~B})$ & $\mathrm{H} 1 \mathrm{c}[1 \mathrm{c} 1 \mathrm{c} 1 \mathrm{~h}]$ & propane \\
\hline $\mathrm{H}(77)$ & $\mathrm{H} 1 \mathrm{c}[1 \mathrm{clc} 1 \mathrm{c}]$ & isobutane \\
\hline $\mathrm{H}(78 \mathrm{~A})$ & $\mathrm{H} 1 \mathrm{c}[\ln 1 \mathrm{c} 1 \mathrm{~h}]$ & ethylamine \\
\hline $\mathrm{H}(78 \mathrm{~B})$ & $\mathrm{H} 1 \mathrm{c}[\ln 1 \mathrm{c} 1 \mathrm{~h}]$ & ethylamine \\
\hline $\mathrm{H}(04)$ & $\mathrm{H} 1 \mathrm{o}[1 \mathrm{c}]$ & methanol \\
\hline $\mathrm{H}(41 \mathrm{~A})$ & $\mathrm{H} 1 \mathrm{c}[1 \mathrm{olh} 1 \mathrm{~h}]$ & methanol \\
\hline $\mathrm{H}(41 \mathrm{~B})$ & $\mathrm{H} 1 \mathrm{c}[1 \mathrm{olh} 1 \mathrm{~h}]$ & methanol \\
\hline $\mathrm{H}(41 \mathrm{C})$ & $\mathrm{H} 1 \mathrm{c}[\mathrm{lolh} 1 \mathrm{~h}]$ & methanol \\
\hline $\mathrm{H}(42 \mathrm{~A})$ & H1o[1h] & water \\
\hline $\mathrm{H}(42 \mathrm{~B})$ & H1o[1h] & water \\
\hline
\end{tabular}

Tabelle A.8: Invariome der asphärischen Verfeinerung von Nadifloxacin $0,5 \mathrm{H}_{2} \mathrm{O}$.

\begin{tabular}{lll} 
Atom & Invariom-Name & Modellverbindung \\
\hline $\mathrm{F}(15)$ & $\mathrm{F} @ 6 \mathrm{c}$ & 1 -fluorobenzene \\
$\mathrm{F}(65)$ & $\mathrm{F} @ 6 \mathrm{c}$ & 1 -fluorobenzene \\
$\mathrm{O}(14)$ & $\mathrm{O} @ 6 \mathrm{c}$ & pyran-4-one $(\gamma$-pyrone) \\
$\mathrm{O}(13)$ & $\mathrm{O} 2 \mathrm{c}$ & formaldehyde \\
$\mathrm{O}(12)$ & $\mathrm{O} 1 \mathrm{c} 1 \mathrm{~h}$ & methanol \\
$\mathrm{O}(23)$ & $\mathrm{O} 1 \mathrm{c} 1 \mathrm{~h}$ & methanol \\
$\mathrm{O}(63)$ & $\mathrm{O} 2 \mathrm{c}$ & formaldehyde \\
$\mathrm{O}(62)$ & $\mathrm{O} 1 \mathrm{c} 1 \mathrm{~h}$ & methanol \\
$\mathrm{O}(73)$ & $\mathrm{O} 1 \mathrm{c} 1 \mathrm{~h}$ & methanol \\
$\mathrm{O}(64)$ & $\mathrm{O} @ 6 \mathrm{c}$ & pyran-4-one $(\gamma$-pyrone) \\
$\mathrm{O}(4)$ & $\mathrm{O} 1 \mathrm{~h} 1 \mathrm{~h}$ & water \\
$\mathrm{N}(1)$ & $6-\mathrm{N} \# 66 \mathrm{c}[\# 66 \mathrm{c} \# 6 \mathrm{c}] \# 6 \mathrm{c}[\# 6 \mathrm{c} 1 \mathrm{~h}] 1 \mathrm{c}$ & 1,3 -dimethylquinolin-4-one \\
$\mathrm{N}(20)$ & $\mathrm{N} @ 6 \mathrm{c} 1 \mathrm{c} 1 \mathrm{c}$ & $N, N$-dimethylbenzenamine \\
$\mathrm{N}(70)$ & $\mathrm{N} @ 6 \mathrm{c} 1 \mathrm{c} 1 \mathrm{c}$ & $N, N$-dimethylbenzenamine \\
$\mathrm{N}(51)$ & $6-\mathrm{N} \# 66 \mathrm{c}[\# 66 \mathrm{c} \# 6 \mathrm{c}] \# 6 \mathrm{c}[\# 6 \mathrm{c} 1 \mathrm{~h}] 1 \mathrm{c}$ & 1,3 -dimethylquinolin-4-one \\
$\mathrm{C}(9)$ & $66-\mathrm{C} \# 6 \mathrm{n}[\# 6 \mathrm{c} 1 \mathrm{c}] \# 66 \mathrm{c}[\# 6 \mathrm{c} \# 6 \mathrm{c}] \# 6 \mathrm{c}[\# 6 \mathrm{c} 1 \mathrm{c}]$ & 2,3 -dihydro-5-oxo-(1H,5H)-benzo[ij]quinolizine \\
$\mathrm{C}(8)$ & $6-\mathrm{C} \# 66 \mathrm{c}[\# 6 \mathrm{n} \# 66 \mathrm{c}] \# 6 \mathrm{c}[\# 6 \mathrm{c} 1 \mathrm{n}] 1 \mathrm{c}$ & 8 -methylquinolin-7-amine \\
\hline
\end{tabular}




\begin{tabular}{|c|c|c|}
\hline Atom & Invariom-Name & Modellverbindung \\
\hline $\mathrm{C}(3)$ & 6-C\#6c[2o\#66c]\#6c[\#6n1h]1c & 3-methylquinolin-4-one \\
\hline $\mathrm{C}(10)$ & 66-C\#66c[\#6n\#6c]\#6c[2o\#6c]\#6c[\#6c1h] & quinolin-4-one \\
\hline $\mathrm{C}(5)$ & 6-C\#66c[\#66c\#6c]\#6c[\#6c1f] $1 \mathrm{~h}$ & 2-fluoronaphthalen \\
\hline $\mathrm{C}(7)$ & 6-C\#6c[\#6c1f]\#6c[\#66c1c]1n & 3-fluoro-1-methylnaphthalen-2-amine \\
\hline $\mathrm{C}(4)$ & 6-C2o\#66c[\#66c\#6c]\#6c[\#6c1c] & 3-methyl-chromen-4-one \\
\hline $\mathrm{C}(6)$ & 6-C\#6c[\#6c1n]\#6c[\#66c1h]lf & 3-fluoronaphthalen-2-amine \\
\hline $\mathrm{C}(11)$ & C2o1o@6c & benzoic acid \\
\hline $\mathrm{C}(16)$ & C@6clc1hlh & isochroman \\
\hline $\mathrm{C}(21)$ & Clnlclhlh & ethylamine \\
\hline $\mathrm{C}(25)$ & Clnlclhlh & ethylamine \\
\hline $\mathrm{C}(22)$ & Clclclhlh & propane \\
\hline $\mathrm{C}(19)$ & Clclhlhlh & ethane \\
\hline $\mathrm{C}(2)$ & 6-C\#6n[\#66c1c]\#6c[\#6c1c]1h & 1,3-dimethylquinolin-4-one \\
\hline $\mathrm{C}(17)$ & Clclclhlh & propane \\
\hline $\mathrm{C}(18)$ & C@6n1c1c1h & 1-isopropylpyridin-2-one \\
\hline $\mathrm{C}(24)$ & Clclclhlh & propane \\
\hline $\mathrm{C}(23)$ & Clolclclh & 2-propanol \\
\hline $\mathrm{C}(59)$ & 66-C\#6n[\#6c1c]\#66c[\#6c\#6c]\#6c[\#6c1c] & 2,3-dihydro-5-oxo-(1H,5H)-benzo[ij]quinolizine \\
\hline $\mathrm{C}(54)$ & 6-C2o\#66c[\#66c\#6c]\#6c[\#6c1c] & 3-methyl-chromen-4-one \\
\hline $\mathrm{C}(57)$ & 6-C\#6c[\#6c1f]\#6c[\#66c1c]1n & 3-fluoro-1-methylnaphthalen-2-amine \\
\hline $\mathrm{C}(55)$ & 6-C\#66c[\#66c\#6c]\#6c[\#6c1f]1h & 2-fluoronaphthalen \\
\hline $\mathrm{C}(53)$ & 6-C\#6c[2o\#66c]\#6c[\#6n1h]1c & 3-methylquinolin-4-one \\
\hline $\mathrm{C}(56)$ & 6-C\#6c[\#6cln]\#6c[\#66c1h]lf & 3-fluoronaphthalen-2-amine \\
\hline $\mathrm{C}(58)$ & 6-C\#66c[\#6n\#66c]\#6c[\#6c1n]1c & 8-methylquinolin-7-amine \\
\hline $\mathrm{C}(60)$ & 66-C\#66c[\#6n\#6c]\#6c[2o\#6c]\#6c[\#6c1h] & quinolin-4-one \\
\hline $\mathrm{C}(68)$ & C@6nlclclh & 1-isopropylpyridin-2-one \\
\hline $\mathrm{C}(66)$ & C@6c1c1h 1h & isochroman \\
\hline $\mathrm{C}(67)$ & Clclclhlh & propane \\
\hline $\mathrm{C}(52)$ & 6-C\#6n[\#66c1c]\#6c[\#6c1c]1h & 1,3-dimethylquinolin-4-one \\
\hline $\mathrm{C}(71)$ & Clnlclhlh & ethylamine \\
\hline $\mathrm{C}(61)$ & C2o1o@6c & benzoic acid \\
\hline $\mathrm{C}(74)$ & Clclclhlh & propane \\
\hline $\mathrm{C}(75)$ & Clnlclhlh & ethylamine \\
\hline $\mathrm{C}(72)$ & Clclclhlh & propane \\
\hline $\mathrm{C}(69)$ & Clclhlhlh & ethane \\
\hline $\mathrm{C}(73)$ & Clolclclh & 2-propanol \\
\hline $\mathrm{H}(12)$ & $\mathrm{H} 1 \mathrm{o}[1 \mathrm{c}]$ & methanol \\
\hline $\mathrm{H}(23)$ & $\mathrm{H} 1 \mathrm{o}[1 \mathrm{c}]$ & methanol \\
\hline $\mathrm{H}(5)$ & $\mathrm{H} @ 6 c$ & benzene \\
\hline $\mathrm{H}(161)$ & H1c[@6c1c1h] & isochroman \\
\hline $\mathrm{H}(162)$ & H1c[@6c1c1h] & isochroman \\
\hline $\mathrm{H}(211)$ & $\mathrm{H} 1 \mathrm{c}[\ln 1 \mathrm{c} 1 \mathrm{~h}]$ & ethylamine \\
\hline $\mathrm{H}(212)$ & $\mathrm{H} 1 \mathrm{c}[\ln 1 \mathrm{c} 1 \mathrm{~h}]$ & ethylamine \\
\hline
\end{tabular}




\begin{tabular}{|c|c|c|}
\hline Atom & Invariom-Name & Modellverbindung \\
\hline $\mathrm{H}(251)$ & $\mathrm{H} 1 \mathrm{c}[\ln 1 \mathrm{c} 1 \mathrm{~h}]$ & ethylamine \\
\hline $\mathrm{H}(252)$ & $\mathrm{H} 1 \mathrm{c}[\ln 1 \mathrm{c} 1 \mathrm{~h}]$ & ethylamine \\
\hline $\mathrm{H}(221)$ & $\mathrm{H} 1 \mathrm{c}[1 \mathrm{c} 1 \mathrm{c} 1 \mathrm{~h}]$ & propane \\
\hline $\mathrm{H}(222)$ & $\mathrm{H} 1 \mathrm{c}[1 \mathrm{c} 1 \mathrm{c} 1 \mathrm{~h}]$ & propane \\
\hline $\mathrm{H}(191)$ & $\mathrm{H} 1 \mathrm{c}[1 \mathrm{c} 1 \mathrm{~h} 1 \mathrm{~h}]$ & ethane \\
\hline $\mathrm{H}(192)$ & $\mathrm{H} 1 \mathrm{c}[1 \mathrm{c} 1 \mathrm{~h} 1 \mathrm{~h}]$ & ethane \\
\hline $\mathrm{H}(193)$ & $\mathrm{H} 1 \mathrm{c}[1 \mathrm{c} 1 \mathrm{~h} 1 \mathrm{~h}]$ & ethane \\
\hline $\mathrm{H}(2)$ & $\mathrm{H} @ 6 \mathrm{c}$ & benzene \\
\hline $\mathrm{H}(171)$ & $\mathrm{H} 1 \mathrm{c}[1 \mathrm{c} 1 \mathrm{c} 1 \mathrm{~h}]$ & propane \\
\hline $\mathrm{H}(172)$ & $\mathrm{H} 1 \mathrm{c}[1 \mathrm{c} 1 \mathrm{c} 1 \mathrm{~h}]$ & propane \\
\hline $\mathrm{H}(18)$ & H1c[@6n1c1c] & 1-isopropylpyridin-2-one \\
\hline $\mathrm{H}(241)$ & $\mathrm{H} 1 \mathrm{c}[1 \mathrm{c} 1 \mathrm{c} 1 \mathrm{~h}]$ & propane \\
\hline $\mathrm{H}(242)$ & $\mathrm{H} 1 \mathrm{c}[1 \mathrm{clc} 1 \mathrm{~h}]$ & propane \\
\hline $\mathrm{H}(231)$ & $\mathrm{H} 1 \mathrm{c}[1 \mathrm{olclc}]$ & 2-propanol \\
\hline $\mathrm{H}(62)$ & $\mathrm{H} 1 \mathrm{o}[1 \mathrm{c}]$ & methanol \\
\hline $\mathrm{H}(73)$ & $\mathrm{H} 1 \mathrm{o}[1 \mathrm{c}]$ & methanol \\
\hline $\mathrm{H}(55)$ & $\mathrm{H} @ 6 c$ & benzene \\
\hline $\mathrm{H}(68)$ & H1c[@6n1c1c] & 1-isopropylpyridin-2-one \\
\hline $\mathrm{H}(661)$ & H1c[@6c1c1h] & isochroman \\
\hline $\mathrm{H}(662)$ & H1c[@6c1c1h] & isochroman \\
\hline $\mathrm{H}(671)$ & $\mathrm{H} 1 \mathrm{c}[1 \mathrm{c} 1 \mathrm{c} 1 \mathrm{~h}]$ & propane \\
\hline $\mathrm{H}(672)$ & $\mathrm{H} 1 \mathrm{c}[1 \mathrm{c} 1 \mathrm{c} 1 \mathrm{~h}]$ & propane \\
\hline $\mathrm{H}(52)$ & $\mathrm{H} @ 6 \mathrm{c}$ & benzene \\
\hline $\mathrm{H}(711)$ & $\mathrm{H} 1 \mathrm{c}[\ln 1 \mathrm{c} 1 \mathrm{~h}]$ & ethylamine \\
\hline $\mathrm{H}(712)$ & $\mathrm{H} 1 \mathrm{c}[\ln 1 \mathrm{c} 1 \mathrm{~h}]$ & ethylamine \\
\hline $\mathrm{H}(741)$ & $\mathrm{H} 1 \mathrm{c}[1 \mathrm{c} 1 \mathrm{c} 1 \mathrm{~h}]$ & propane \\
\hline $\mathrm{H}(742)$ & $\mathrm{H} 1 \mathrm{c}[1 \mathrm{clc} 1 \mathrm{~h}]$ & propane \\
\hline $\mathrm{H}(751)$ & $\mathrm{H} 1 \mathrm{c}[\ln 1 \mathrm{c} 1 \mathrm{~h}]$ & ethylamine \\
\hline $\mathrm{H}(752)$ & $\mathrm{H} 1 \mathrm{c}[\ln 1 \mathrm{c} 1 \mathrm{~h}]$ & ethylamine \\
\hline $\mathrm{H}(721)$ & $\mathrm{H} 1 \mathrm{c}[1 \mathrm{c} 1 \mathrm{c} 1 \mathrm{~h}]$ & propane \\
\hline $\mathrm{H}(722)$ & $\mathrm{H} 1 \mathrm{c}[1 \mathrm{c} 1 \mathrm{c} 1 \mathrm{~h}]$ & propane \\
\hline $\mathrm{H}(691)$ & $\mathrm{H} 1 \mathrm{c}[1 \mathrm{c} 1 \mathrm{~h} 1 \mathrm{~h}]$ & ethane \\
\hline $\mathrm{H}(692)$ & $\mathrm{H} 1 \mathrm{c}[1 \mathrm{c} 1 \mathrm{~h} 1 \mathrm{~h}]$ & ethane \\
\hline $\mathrm{H}(693)$ & $\mathrm{H} 1 \mathrm{c}[1 \mathrm{c} 1 \mathrm{~h} 1 \mathrm{~h}]$ & ethane \\
\hline $\mathrm{H}(731)$ & $\mathrm{H} 1 \mathrm{c}[1 \mathrm{olclc}]$ & 2-propanol \\
\hline $\mathrm{H}(41)$ & H1o[1h] & water \\
\hline $\mathrm{H}(42)$ & H1o[1h] & water \\
\hline
\end{tabular}

Tabelle A.9: Invariome der asphärischen Verfeinerung von NorfloxacinAnhydrat. 


\begin{tabular}{|c|c|c|}
\hline Atom & Invariom-Name & Modellverbindung \\
\hline $\mathrm{F}(15)$ & $\mathrm{F} @ 6 \mathrm{c}$ & 1-fluorobenzene \\
\hline $\mathrm{O}(12)$ & $\mathrm{O} 2 \mathrm{c}$ & formaldehyde \\
\hline $\mathrm{O}(13)$ & Olclh & methanol \\
\hline $\mathrm{O}(14)$ & O@6c & pyran-4-one ( $\gamma$-pyrone) \\
\hline $\mathrm{N}(1)$ & $6-\mathrm{N} \# 66 \mathrm{c}[\# 66 \mathrm{c} \# 6 \mathrm{c}] \# 6 \mathrm{c}[\# 6 \mathrm{c} 1 \mathrm{~h}] 1 \mathrm{c}$ & 1,3-dimethylquinolin-4-one \\
\hline $\mathrm{N}(20)$ & $\mathrm{N} @ 6 c 1 \mathrm{c} 1 \mathrm{c}$ & $N, N$-dimethylbenzenamine \\
\hline $\mathrm{N}(23)$ & N1clclh & dimethylamine \\
\hline $\mathrm{C}(2)$ & 6-C\#6n[\#66c1c]\#6c[\#6c1c]1h & 1,3-dimethylquinolin-4-one \\
\hline $\mathrm{C}(3)$ & $6-\mathrm{C} \# 6 \mathrm{c}[2 \mathrm{o} \# 66 \mathrm{c}] \# 6 \mathrm{c}[\# 6 \mathrm{n} 1 \mathrm{~h}] 1 \mathrm{c}$ & 3-methylquinolin-4-one \\
\hline $\mathrm{C}(11)$ & C2o1o@6c & benzoic acid \\
\hline $\mathrm{C}(4)$ & 6-C2o\#66c[\#66c\#6c]\#6c[\#6c1c] & 3-methyl-chromen-4-one \\
\hline$C(10)$ & 66-C\#66c[\#6n\#6c]\#6c[2o\#6c]\#6c[\#6c1h] & quinolin-4-one \\
\hline $\mathrm{C}(5)$ & 6-C\#66c[\#66c\#6c]\#6c[\#6c1f] $1 \mathrm{~h}$ & 2-fluoronaphthalen \\
\hline $\mathrm{C}(6)$ & 6-C\#6c[\#6c1n]\#6c[\#66c1h]1f & 3-fluoronaphthalen-2-amine \\
\hline $\mathrm{C}(7)$ & 6-C\#6c[\#6c1f]\#6c[\#66clh] $\ln$ & 3-fluoronaphthalen-2-amine \\
\hline $\mathrm{C}(8)$ & 6-C\#66c[\#6n\#66c]\#6c[\#6c1n]1h & 7-aminoquinolin-2-one \\
\hline $\mathrm{C}(9)$ & 66-C\#6n[\#6c1c]\#66c[\#6c\#6c]\#6c[\#6c1h] & 1,3-dimethylquinolin-4-one \\
\hline$C(25)$ & Clnlclhlh & ethylamine \\
\hline $\mathrm{C}(24)$ & Clnlclhlh & ethylamine \\
\hline $\mathrm{C}(22)$ & Clnlclhlh & ethylamine \\
\hline $\mathrm{C}(21)$ & Clnlclhlh & ethylamine \\
\hline$C(16)$ & C@6n1c1h1h & 1-ethylpylpyridine-2-one \\
\hline $\mathrm{C}(17)$ & Clclhlh1h & ethane \\
\hline $\mathrm{H}(13)$ & $\mathrm{H} 1 \mathrm{o}[1 \mathrm{c}]$ & methanol \\
\hline $\mathrm{H}(23 \mathrm{~A})$ & $\mathrm{H} \ln [1 \mathrm{c} 1 \mathrm{c}]$ & dimethylamine \\
\hline $\mathrm{H}(2)$ & $\mathrm{H} @ 6 \mathrm{c}$ & benzene \\
\hline $\mathrm{H}(5)$ & $\mathrm{H} @ 6 c$ & benzene \\
\hline $\mathrm{H}(8)$ & $\mathrm{H} @ 6 c$ & benzene \\
\hline $\mathrm{H}(25 \mathrm{~A})$ & $\mathrm{H} 1 \mathrm{c}[\ln 1 \mathrm{c} 1 \mathrm{~h}]$ & ethylamine \\
\hline$H(25 B)$ & $\mathrm{H} 1 \mathrm{c}[\ln 1 \mathrm{c} 1 \mathrm{~h}]$ & ethylamine \\
\hline $\mathrm{H}(24 \mathrm{~A})$ & $\mathrm{H} 1 \mathrm{c}[\ln 1 \mathrm{c} 1 \mathrm{~h}]$ & ethylamine \\
\hline $\mathrm{H}(24 \mathrm{~B})$ & $\mathrm{H} 1 \mathrm{c}[\ln 1 \mathrm{c} 1 \mathrm{~h}]$ & ethylamine \\
\hline $\mathrm{H}(22 \mathrm{~A})$ & $\mathrm{H} 1 \mathrm{c}[\ln 1 \mathrm{c} 1 \mathrm{~h}]$ & ethylamine \\
\hline $\mathrm{H}(22 \mathrm{~B})$ & $\mathrm{H} 1 \mathrm{c}[\ln 1 \mathrm{c} 1 \mathrm{~h}]$ & ethylamine \\
\hline $\mathrm{H}(21 \mathrm{~A})$ & $\mathrm{H} 1 \mathrm{c}[\ln 1 \mathrm{c} 1 \mathrm{~h}]$ & ethylamine \\
\hline $\mathrm{H}(21 \mathrm{~B})$ & $\mathrm{H} 1 \mathrm{c}[\ln 1 \mathrm{c} 1 \mathrm{~h}]$ & ethylamine \\
\hline $\mathrm{H}(16 \mathrm{~A})$ & H1c[@6n1c1h] & 1-ethylpylpyridine-2-one \\
\hline $\mathrm{H}(16 \mathrm{~B})$ & H1c[@6n1c1h] & 1-ethylpylpyridine-2-one \\
\hline $\mathrm{H}(17 \mathrm{~A})$ & $\mathrm{H} 1 \mathrm{c}[1 \mathrm{c} 1 \mathrm{~h} 1 \mathrm{~h}]$ & ethane \\
\hline $\mathrm{H}(17 \mathrm{~B})$ & $\mathrm{H} 1 \mathrm{c}[1 \mathrm{c} 1 \mathrm{~h} 1 \mathrm{~h}]$ & ethane \\
\hline $\mathrm{H}(17 \mathrm{C})$ & $\mathrm{H} 1 \mathrm{c}[\mathrm{lc} \operatorname{ch} 1 \mathrm{~h}]$ & ethane \\
\hline
\end{tabular}


Tabelle A.10: Invariome der asphärischen Verfeinerung von Norfloxacin $2 \mathrm{H}_{2} \mathrm{O}$.

\begin{tabular}{|c|c|c|}
\hline Atom & Invariom-Name & Modellverbindung \\
\hline $\mathrm{F}(15)$ & $\mathrm{F} @ 6 \mathrm{c}$ & 1-fluorobenzene \\
\hline $\mathrm{O}(13)$ & O1.5c[1.5o@6c] & benzoic acid anion \\
\hline $\mathrm{O}(12)$ & O1.5c[1.5o@6c] & benzoic acid anion \\
\hline $\mathrm{O}(14)$ & O@6c & pyran-4-one ( $\gamma$-pyrone) \\
\hline $\mathrm{O}(2)$ & Olhlh & water \\
\hline $\mathrm{O}(3)$ & Olhlh & water \\
\hline $\mathrm{N}(23)$ & N1clc1h1h & dimethylammonium \\
\hline $\mathrm{N}(1)$ & $6-\mathrm{N} \# 66 \mathrm{c}[\# 66 \mathrm{c} \# 6 \mathrm{c}] \# 6 \mathrm{c}[\# 6 \mathrm{c} 1 \mathrm{~h}] 1 \mathrm{c}$ & 1,3-dimethylquinolin-4-one \\
\hline $\mathrm{N}(20)$ & $\mathrm{N} @ 6 c 1 c 1 c$ & $N, N$-dimethylbenzenamine \\
\hline $\mathrm{C}(6)$ & 6-C\#6c[\#6cln]\#6c[\#66c1h]1f & 3-fluoronaphthalen-2-amine \\
\hline $\mathrm{C}(11)$ & C1.5o1.5o@6c & benzoic acid anion \\
\hline $\mathrm{C}(4)$ & 6-C2o\#66c[\#66c\#6c]\#6c[\#6c1c] & 3-methyl-chromen-4-one \\
\hline $\mathrm{C}(3)$ & 6-C\#6c[2o\#66c]\#6c[\#6n 1h]1c & 3-methylquinolin-4-one \\
\hline$C(10)$ & 66-C\#66c[\#6n\#6c]\#6c[2o\#6c]\#6c[\#6c1h] & quinolin-4-one \\
\hline $\mathrm{C}(5)$ & 6-C\#66c[\#66c\#6c]\#6c[\#6c1f]1h & 2-fluoronaphthalen \\
\hline $\mathrm{C}(2)$ & 6-C\#6n[\#66c1c]\#6c[\#6c1c]1h & 1,3-dimethylquinolin-4-one \\
\hline $\mathrm{C}(9)$ & 66-C\#6n[\#6c1c]\#66c[\#6c\#6c]\#6c[\#6c1h] & 1,3-dimethylquinolin-4-one \\
\hline $\mathrm{C}(8)$ & 6-C\#66c[\#6n\#66c]\#6c[\#6c1n]1h & 7-aminoquinolin-2-one \\
\hline $\mathrm{C}(7)$ & 6-C\#6c[\#6c1f]\#6c[\#66c1h]1n & 3-fluoronaphthalen-2-amine \\
\hline$C(16)$ & C@6n1c1h1h & 1-ethylpylpyridine-2-one \\
\hline $\mathrm{C}(25)$ & Cln1clh1h & ethylamine \\
\hline $\mathrm{C}(21)$ & Clnlclhlh & ethylamine \\
\hline $\mathrm{C}(24)$ & Clnlclhlh & ethylamine \\
\hline $\mathrm{C}(22)$ & Clnlclhlh & ethylamine \\
\hline $\mathrm{C}(17)$ & Clclhlhlh & ethane \\
\hline $\mathrm{H}(23 \mathrm{~A})$ & $\mathrm{H} \ln [1 \mathrm{c} 1 \mathrm{c} 1 \mathrm{~h}]$ & dimethylammonium \\
\hline $\mathrm{H}(23 \mathrm{~B})$ & $\mathrm{H} \ln [1 \mathrm{c} 1 \mathrm{c} 1 \mathrm{~h}]$ & dimethylammonium \\
\hline $\mathrm{H}(5)$ & $\mathrm{H} @ 6 \mathrm{c}$ & benzene \\
\hline $\mathrm{H}(2)$ & H@6c & benzene \\
\hline $\mathrm{H}(8)$ & $\mathrm{H} @ 6 c$ & benzene \\
\hline $\mathrm{H}(16 \mathrm{~A})$ & H1c[@6n1c1h] & 1-ethylpylpyridine-2-one \\
\hline $\mathrm{H}(16 \mathrm{~B})$ & H1c[@6n1c1h] & 1-ethylpylpyridine-2-one \\
\hline $\mathrm{H}(25 \mathrm{~A})$ & $\mathrm{H} 1 \mathrm{c}[\ln 1 \mathrm{c} 1 \mathrm{~h}]$ & ethylamine \\
\hline $\mathrm{H}(25 \mathrm{~B})$ & $\mathrm{H} 1 \mathrm{c}[\ln 1 \mathrm{c} 1 \mathrm{~h}]$ & ethylamine \\
\hline $\mathrm{H}(21 \mathrm{~A})$ & $\mathrm{H} 1 \mathrm{c}[\ln 1 \mathrm{c} 1 \mathrm{~h}]$ & ethylamine \\
\hline $\mathrm{H}(21 \mathrm{~B})$ & $\mathrm{H} 1 \mathrm{c}[\ln 1 \mathrm{c} 1 \mathrm{~h}]$ & ethylamine \\
\hline $\mathrm{H}(24 \mathrm{~A})$ & $\mathrm{H} 1 \mathrm{c}[\ln 1 \mathrm{c} 1 \mathrm{~h}]$ & ethylamine \\
\hline $\mathrm{H}(24 \mathrm{~B})$ & $\mathrm{H} 1 \mathrm{c}[\ln 1 \mathrm{c} 1 \mathrm{~h}]$ & ethylamine \\
\hline $\mathrm{H}(22 \mathrm{~A})$ & $\mathrm{H} 1 \mathrm{c}[\ln 1 \mathrm{c} 1 \mathrm{~h}]$ & ethylamine \\
\hline $\mathrm{H}(22 \mathrm{~B})$ & $\mathrm{H} 1 \mathrm{c}[\ln 1 \mathrm{c} 1 \mathrm{~h}]$ & ethylamine \\
\hline
\end{tabular}




\begin{tabular}{lll} 
Atom & Invariom-Name & Modellverbindung \\
\hline $\mathrm{H}(17 \mathrm{~A})$ & $\mathrm{H} 1 \mathrm{c}[1 \mathrm{c} 1 \mathrm{~h} 1 \mathrm{~h}]$ & ethane \\
$\mathrm{H}(17 \mathrm{~B})$ & $\mathrm{H} 1 \mathrm{c}[1 \mathrm{c} 1 \mathrm{~h} 1 \mathrm{~h}]$ & ethane \\
$\mathrm{H}(17 \mathrm{C})$ & $\mathrm{H} 1 \mathrm{c}[1 \mathrm{c} 1 \mathrm{~h} 1 \mathrm{~h}]$ & ethane \\
$\mathrm{H}(021)$ & $\mathrm{H} 1 \mathrm{o}[1 \mathrm{~h}]$ & water \\
$\mathrm{H}(022)$ & $\mathrm{H} 1 \mathrm{o}[1 \mathrm{~h}]$ & water \\
$\mathrm{H}(031)$ & $\mathrm{H} 1 \mathrm{o}[1 \mathrm{~h}]$ & water \\
$\mathrm{H}(032)$ & $\mathrm{H} 1 \mathrm{o}[1 \mathrm{~h}]$ & water \\
\hline
\end{tabular}

Tabelle A.11: Invariome der asphärischen Verfeinerung von OfloxacinAnhydrat.

\begin{tabular}{|c|c|c|}
\hline Atom & Invariom-Name & Modellverbindung \\
\hline $\mathrm{F}(15)$ & F@6c & 1-fluorobenzene \\
\hline $\mathrm{O}(13)$ & $\mathrm{O} 2 \mathrm{c}$ & formaldehyde \\
\hline $\mathrm{O}(14)$ & $0 @ 6 c$ & pyran-4-one ( $\gamma$-pyrone) \\
\hline $\mathrm{O}(12)$ & Olclh & methanol \\
\hline $\mathrm{O}(16)$ & $0 @ 6 c 1 c$ & anisole \\
\hline $\mathrm{N}(1)$ & 6-N\#66c[\#66c\#6c]\#6c[\#6c1h]1c & 1,3-dimethylquinolin-4-one \\
\hline $\mathrm{N}(20)$ & $\mathrm{N} @ 6 \mathrm{c} 1 \mathrm{c} 1 \mathrm{c}$ & $\mathrm{N}, \mathrm{N}$-dimethylbenzenamine \\
\hline $\mathrm{N}(23)$ & Nlclclc & trimethylamine \\
\hline $\mathrm{C}(2)$ & $6-\mathrm{C} \# 6 \mathrm{n}[\# 66 \mathrm{c} 1 \mathrm{c}] \# 6 \mathrm{c}[\# 6 \mathrm{c} 1 \mathrm{c}] 1 \mathrm{~h}$ & 1,3-dimethylquinolin-4-one \\
\hline $\mathrm{C}(9)$ & $66-C \# 6 n[\# 6 c 1 c] \# 66 c[\# 6 c \# 6 c] \# 6 c[\# 6 c 10]$ & 8-hydroxy-1-methylquinolin-2-one \\
\hline $\mathrm{C}(10)$ & $66-\mathrm{C} \# 66 \mathrm{c}[\# 6 \mathrm{n} \# 6 \mathrm{cc}] \# 6 \mathrm{cc}[2 \mathrm{o} \# 6 \mathrm{c}] \# 6 \mathrm{c}[\# 6 \mathrm{c} 1 \mathrm{~h}]$ & quinolin-4-one \\
\hline $\mathrm{C}(11)$ & C2olo@6c & benzoic acid \\
\hline $\mathrm{C}(3)$ & $6-\mathrm{C} \# 6 \mathrm{c}[2 \mathrm{o} \# 66 \mathrm{c}] \# 6 \mathrm{c}[\# 6 \mathrm{n} 1 \mathrm{~h}] 1 \mathrm{c}$ & 3-methylquinolin-4-one \\
\hline $\mathrm{C}(6)$ & $6-\mathrm{C} \# 6 c[\# 6 \mathrm{c} 1 \mathrm{n}] \# 6 \mathrm{cc}[\# 66 \mathrm{c} 1 \mathrm{~h}] 1 \mathrm{f}$ & 3-fluoronaphthalen-2-amine \\
\hline $\mathrm{C}(5)$ & 6-C\#66c[\#66c\#6c]\#6c[\#6c1f]1h & 2-fluoronaphthalen \\
\hline $\mathrm{C}(7)$ & 6-C\#6c[\#6c1f]\#6c[\#66c1o]1n & 2-amino-3-fluoronaphthalen-1-ol \\
\hline $\mathrm{C}(8)$ & 6-C\#66c[\#6n\#66c]\#6c[\#6c1n]1o & 7-amino-8-hydroxyquinolin-2-one \\
\hline $\mathrm{C}(4)$ & 6-C2o\#66c[\#66c\#6c] \#6c[\#6c1c] & 3-methyl-chromen-4-one \\
\hline $\mathrm{C}(24)$ & Clnlclhlh & ethylamine \\
\hline$C(21)$ & Clnlclhlh & ethylamine \\
\hline $\mathrm{C}(22)$ & Clnlclhlh & ethylamine \\
\hline$C(18)$ & C@6n1clc1h & 1-isopropylpyridin-2-one \\
\hline $\mathrm{C}(17)$ & Clolclhlh & ethanol \\
\hline $\mathrm{C}(19)$ & Clclhlhlh & ethane \\
\hline $\mathrm{C}(26)$ & Clnlhlhlh & methylamine \\
\hline $\mathrm{C}(25)$ & Clnlclhlh & ethylamine \\
\hline $\mathrm{H}(2)$ & H@6c & benzene \\
\hline $\mathrm{H}(12)$ & $\mathrm{H} l \mathrm{o}[\mathrm{lc}]$ & methanol \\
\hline
\end{tabular}




\begin{tabular}{lll} 
Atom & Invariom-Name & Modellverbindung \\
\hline $\mathrm{H}(5)$ & $\mathrm{H} @ 6 \mathrm{c}$ & benzene \\
$\mathrm{H}(24 \mathrm{~A})$ & $\mathrm{H} 1 \mathrm{c}[\ln 1 \mathrm{c} 1 \mathrm{~h}]$ & ethylamine \\
$\mathrm{H}(24 \mathrm{~B})$ & $\mathrm{H} 1 \mathrm{c}[\ln 1 \mathrm{c} 1 \mathrm{~h}]$ & ethylamine \\
$\mathrm{H}(21 \mathrm{~A})$ & $\mathrm{H} 1 \mathrm{c}[\ln 1 \mathrm{c} 1 \mathrm{~h}]$ & ethylamine \\
$\mathrm{H}(21 \mathrm{~B})$ & $\mathrm{H} 1 \mathrm{c}[\ln 1 \mathrm{c} 1 \mathrm{~h}]$ & ethylamine \\
$\mathrm{H}(22 \mathrm{~A})$ & $\mathrm{H} 1 \mathrm{c}[\ln 1 \mathrm{c} 1 \mathrm{~h}]$ & ethylamine \\
$\mathrm{H}(22 \mathrm{~B})$ & $\mathrm{H} 1 \mathrm{c}[\ln 1 \mathrm{c} 1 \mathrm{~h}]$ & ethylamine \\
$\mathrm{H}(18)$ & $\mathrm{H} 1 \mathrm{c}[6 \mathrm{n} 1 \mathrm{c} 1 \mathrm{c}]$ & 1 -isopropylpyridin-2-one \\
$\mathrm{H}(17 \mathrm{~A})$ & $\mathrm{H} 1 \mathrm{c}[1 \mathrm{lol} 1 \mathrm{~h}]$ & ethanol \\
$\mathrm{H}(17 \mathrm{~B})$ & $\mathrm{H} 1 \mathrm{c}[1 \mathrm{lol} 1 \mathrm{~h}]$ & ethanol \\
$\mathrm{H}(19 \mathrm{~A})$ & $\mathrm{H} 1 \mathrm{c}[1 \mathrm{c} 1 \mathrm{~h} 1 \mathrm{~h}]$ & ethane \\
$\mathrm{H}(19 \mathrm{~B})$ & $\mathrm{H} 1 \mathrm{c}[1 \mathrm{c} 1 \mathrm{~h} 1 \mathrm{~h}]$ & ethane \\
$\mathrm{H}(19 \mathrm{C})$ & $\mathrm{H} 1 \mathrm{c}[1 \mathrm{c} 1 \mathrm{~h} 1 \mathrm{~h}]$ & ethane \\
$\mathrm{H}(26 \mathrm{~A})$ & $\mathrm{H} 1 \mathrm{c}[\ln 1 \mathrm{~h} 1 \mathrm{~h}]$ & methylamine \\
$\mathrm{H}(26 \mathrm{~B})$ & $\mathrm{H} 1 \mathrm{c}[\ln 1 \mathrm{~h} 1 \mathrm{~h}]$ & methylamine \\
$\mathrm{H}(26 \mathrm{C})$ & $\mathrm{H} 1 \mathrm{c}[\ln 1 \mathrm{~h} 1 \mathrm{~h}]$ & methylamine \\
$\mathrm{H}(25 \mathrm{~A})$ & $\mathrm{H} 1 \mathrm{c}[\ln 1 \mathrm{c} 1 \mathrm{~h}]$ & ethylamine \\
$\mathrm{H}(25 \mathrm{~B})$ & $\mathrm{H} 1 \mathrm{c}[\ln 1 \mathrm{c} 1 \mathrm{~h}]$ & ethylamine \\
\hline
\end{tabular}

Tabelle A.12: Invariome der asphärischen Verfeinerung von Sparfloxacin $3 \mathrm{H}_{2} \mathrm{O}$.

\begin{tabular}{|c|c|c|}
\hline Atom & Invariom-Name & Modellverbindung \\
\hline $\mathrm{F}(8)$ & $\mathrm{F} @ 6 \mathrm{c}$ & 1-fluorobenzene \\
\hline$F(15)$ & $\mathrm{F} @ 6 c$ & 1-fluorobenzene \\
\hline $\mathrm{O}(12)$ & O1.5c[1.5o@6c] & benzoic acid anion \\
\hline $\mathrm{O}(13)$ & O1.5c[1.5o@6c] & benzoic acid anion \\
\hline $\mathrm{O}(14)$ & $0 @ 6 c$ & pyran-4-one ( $\gamma$-pyrone) \\
\hline $\mathrm{O}(2)$ & Olhlh & water \\
\hline $\mathrm{O}(3)$ & Olh1h & water \\
\hline $\mathrm{O}(4)$ & Olhlh & water \\
\hline $\mathrm{N}(1)$ & 6-N\#66c[\#66c\#6c]\#6c[\#6c1h]@3c & 1-cyclopropylquinolin-4-one \\
\hline $\mathrm{N}(5)$ & $=-\mathrm{N} @ 6 \mathrm{c} 1 \mathrm{~h} 1 \mathrm{~h}$ & 4-aminopyrimidin-2-one \\
\hline $\mathrm{N}(20)$ & $\mathrm{N} @ 6 \mathrm{c} 1 \mathrm{c} 1 \mathrm{c}$ & $N, N$-dimethylbenzenamine \\
\hline $\mathrm{N}(23)$ & N1clclhlh & dimethylammonium \\
\hline $\mathrm{C}(2)$ & 6-C\#6n[\#66c@3c]\#6c[\#6c1c]1h & 1-cyclopropyl-3-methylquinolin-4-one \\
\hline $\mathrm{C}(3)$ & 6-C\#6c[2o\#66c]\#6c[\#6n1h]1c & 3-methylquinolin-4-one \\
\hline $\mathrm{C}(4)$ & 6-C2o\#66c[\#66c\#6c]\#6c[\#6c1c] & 3-methyl-chromen-4-one \\
\hline $\mathrm{C}(10)$ & 66-C\#66c[\#6n\#6c]\#6c[2o\#6c]\#6c[\#6c1n] & 5-aminoquinolin-4-one \\
\hline $\mathrm{C}(9)$ & 66-C\#6n[\#6c@3c]\#66c[\#6c\#6c]\#6c[\#6c1f] & 1-cyclopropyl-8-fluoroquinolin-2-one \\
\hline $\mathrm{C}(8)$ & 6-C\#66c[\#6n\#66c]\#6c[\#6cln]lf & 7-amino-8-fluoroquinolin-2-one \\
\hline
\end{tabular}




\begin{tabular}{|c|c|c|}
\hline Atom & Invariom-Name & Modellverbindung \\
\hline $\mathrm{C}(7)$ & 6-C\#6c[\#66c1f]\#6c[\#6c1f] $1 \mathrm{n}$ & 1,3-difluoronaphthalen-2-amine \\
\hline $\mathrm{C}(6)$ & $6-\mathrm{C} \# 6 \mathrm{c}[\# 66 \mathrm{c} \ln ] \# 6 \mathrm{c}[\# 6 \mathrm{c} \ln ] 1 \mathrm{f}$ & 2-fluoronaphthalene-1,3-diamine \\
\hline $\mathrm{C}(5)$ & 6-C\#66c[\#66c\#6c]\#6c[\#6c1f] $1 \mathrm{n}$ & 2-fluoronaphthalen-1-amine \\
\hline $\mathrm{C}(21)$ & Cln $1 \mathrm{c} 1 \mathrm{~h} 1 \mathrm{~h}$ & ethylamine \\
\hline $\mathrm{C}(22)$ & Cln $1 \mathrm{clc} 1 \mathrm{~h}$ & 2-aminopropane \\
\hline $\mathrm{C}(24)$ & Cln $1 \mathrm{c} 1 \mathrm{c} 1 \mathrm{~h}$ & 2-aminopropane \\
\hline$C(25)$ & Clnlclhlh & ethylamine \\
\hline $\mathrm{C}(27)$ & Clclhlhlh & ethane \\
\hline $\mathrm{C}(26)$ & Clclhlhlh & ethane \\
\hline$C(16)$ & 3-C\#3c[\#3c1h1h]\#3c[\#3c1h1h]@6n1h & 1-cyclopropylpyridine-2-one \\
\hline $\mathrm{C}(17)$ & 3-C\#3c[\#3c@6n1h]\#3c[\#3c1h1h]1h1h & 1-cyclopropylpyridine-2-one \\
\hline $\mathrm{C}(18)$ & 3-C\#3c[\#3c@6n1h]\#3c[\#3c1h1h]1h1h & 1-cyclopropylpyridine-2-one \\
\hline $\mathrm{C}(11)$ & C1.5o1.5o@6c & benzoic acid anion \\
\hline $\mathrm{H}(52)$ & H1n[@6c1h] & aniline \\
\hline $\mathrm{H}(51)$ & H1n[@6c1h] & aniline \\
\hline $\mathrm{H}(23 \mathrm{~A})$ & $\mathrm{H} \ln [1 \mathrm{c} 1 \mathrm{c} 1 \mathrm{~h}]$ & dimethylammonium \\
\hline $\mathrm{H}(23 \mathrm{~B})$ & $\mathrm{H} \ln [1 \mathrm{c} 1 \mathrm{c} 1 \mathrm{~h}]$ & dimethylammonium \\
\hline $\mathrm{H}(2)$ & $\mathrm{H} @ 6 \mathrm{c}$ & benzene \\
\hline $\mathrm{H}(21 \mathrm{~A})$ & $\mathrm{H} 1 \mathrm{c}[\ln 1 \mathrm{c} 1 \mathrm{~h}]$ & ethylamine \\
\hline $\mathrm{H}(21 \mathrm{~B})$ & $\mathrm{H} 1 \mathrm{c}[\ln 1 \mathrm{c} 1 \mathrm{~h}]$ & ethylamine \\
\hline $\mathrm{H}(22 \mathrm{~A})$ & $\mathrm{H} 1 \mathrm{c}[\ln 1 \mathrm{c} 1 \mathrm{c}]$ & 2-aminopropane \\
\hline $\mathrm{H}(24 \mathrm{~A})$ & $\mathrm{H} 1 \mathrm{c}[\ln 1 \mathrm{c} 1 \mathrm{c}]$ & 2-aminopropane \\
\hline $\mathrm{H}(251)$ & $\mathrm{H} 1 \mathrm{c}[\ln 1 \mathrm{c} 1 \mathrm{~h}]$ & ethylamine \\
\hline $\mathrm{H}(252)$ & $\mathrm{H} 1 \mathrm{c}[\ln 1 \mathrm{c} 1 \mathrm{~h}]$ & ethylamine \\
\hline $\mathrm{H}(27 \mathrm{~A})$ & $\mathrm{H} 1 \mathrm{c}[1 \mathrm{c} 1 \mathrm{~h} 1 \mathrm{~h}]$ & ethane \\
\hline $\mathrm{H}(27 \mathrm{~B})$ & $\mathrm{H} 1 \mathrm{c}[1 \mathrm{c} 1 \mathrm{~h} 1 \mathrm{~h}]$ & ethane \\
\hline $\mathrm{H}(27 \mathrm{C})$ & $\mathrm{H} 1 \mathrm{c}[1 \mathrm{c} 1 \mathrm{~h} 1 \mathrm{~h}]$ & ethane \\
\hline $\mathrm{H}(26 \mathrm{~A})$ & $\mathrm{H} 1 \mathrm{c}[1 \mathrm{c} 1 \mathrm{~h} 1 \mathrm{~h}]$ & ethane \\
\hline $\mathrm{H}(26 \mathrm{~B})$ & $\mathrm{H} 1 \mathrm{c}[1 \mathrm{c} 1 \mathrm{~h} 1 \mathrm{~h}]$ & ethane \\
\hline $\mathrm{H}(26 \mathrm{C})$ & $\mathrm{H} 1 \mathrm{c}[1 \mathrm{c} 1 \mathrm{~h} 1 \mathrm{~h}]$ & ethane \\
\hline $\mathrm{H}(16)$ & $\mathrm{H} @ 3 c$ & cyclopropane \\
\hline $\mathrm{H}(17 \mathrm{~A})$ & $\mathrm{H} @ 3 \mathrm{c}$ & cyclopropane \\
\hline $\mathrm{H}(17 \mathrm{~B})$ & $\mathrm{H} @ 3 \mathrm{c}$ & cyclopropane \\
\hline $\mathrm{H}(18 \mathrm{~A})$ & $\mathrm{H} @ 3 \mathrm{c}$ & cyclopropane \\
\hline $\mathrm{H}(18 \mathrm{~B})$ & $\mathrm{H} @ 3 c$ & cyclopropane \\
\hline $\mathrm{H}(021)$ & $\mathrm{H} 1 \mathrm{o}[\mathrm{lh}]$ & water \\
\hline $\mathrm{H}(022)$ & $\mathrm{H} 1 \mathrm{o}[1 \mathrm{~h}]$ & water \\
\hline $\mathrm{H}(031)$ & $\mathrm{H} 1 \mathrm{o}[1 \mathrm{~h}]$ & water \\
\hline $\mathrm{H}(032)$ & $\mathrm{H} 1 \mathrm{o}[\mathrm{lh}]$ & water \\
\hline $\mathrm{H}(041)$ & $\mathrm{H} 1 \mathrm{lo}[\mathrm{lh}]$ & water \\
\hline $\mathrm{H}(042)$ & $\mathrm{H} 1 \mathrm{o}[1 \mathrm{~h}]$ & water \\
\hline
\end{tabular}




\section{Danksagung}

Dr. Birger Dittrich danke ich für die Betreuung meiner Doktorarbeit, die Unterstützung bei Messungen und Veröffentlichungen sowie die zahlreichen Möglichkeiten meine Forschungsergebnisse auf nationalen und internationalen Konferenzen zu präsentieren. Prof. George M. Sheldrick gilt mein Dank für die vielfältigen thematischen Einblicke in die Kristallographie, die Möglichkeit am Diffraktometer der Arbeitsgruppe zu messen sowie die Übernahme des Referats dieser Arbeit.

Dr. Christian B. Hübschle danke ich für die gute Stimmung im Büro, eine sehr fruchtbare Zusammenarbeit, die Programme MolecoolQT und SHELXlE und zahlreiche Diskussionen. Kevin Pröpper gilt mein Dank für die gute Kooperation in den gemeinsamen Projekten. Dr. Tim Grüne, Dr. Regine HerbstIrmer und Daniel Holstein danke ich für das Korrekturlesen dieser Arbeit und kritische Anmerkungen. Dr. Andrea Thorn und Dr. Francesca P. A. Fabbiani, Dr. Christian Große, Dr. Tobias Beck und Navdeep Sidhu danke ich für die hilfreichen Diskussionen und die sehr gute Arbeitsatmosphäre.

Prof. Dr. Dietmar Stalke danke ich für die Möglichkeit an den Diffraktometern seiner Arbeitsgruppe zu messen. Helmut Dehnhardt gilt mein Dank für die Wartung des Diffraktometers. Den Mitarbeitern der Werkstätten und den Hausmeistern möchte ich für logistische und technische Unterstützung bei der Restrukturierung des AC1-Praktikums danken. Dr. A. Claudia Stückl, Peri Linne und Petra Gaugel danke ich für die Hilfe bei bürokratischen Fragen.

Mein herzlicher Dank für die Unterstützung in der Studien- und Promotionszeit gilt meiner Familie. 


\section{Publikationsliste}

\section{Veröffentlichungen in wissenschaftlichen Journalen}

1. B. Dittrich, C. B. Hübschle, J. J. Holstein und F. P. A. Fabbiani „Towards extracting the charge density from normal-resolution data“ J. Appl. Cryst. 42 (2009), 1110-1121

2. J. J. Holstein, P. Luger, R. Kalinowski, S. Mebs, C. Paulmann und B. Dittrich „Validation of experimental charge densities: refinement of the macrolide antibiotic roxithromycin“ Acta Cryst. B 66 (2010), 568-577

3. R. S. Ghadwal, R. Azhakar, K. Pröpper, J. J. Holstein, B. Dittrich und H. Roesky „N-Heterocyclic carbene stabilized dichlorosilylene transitionmetal complexes of $\mathrm{V}(\mathrm{I}), \mathrm{Co}(\mathrm{I})$, and $\mathrm{Fe}(0) “$ Inorg. Chem. 50 (2011), 85028508

4. J. J. Holstein, C. B. Hübschle und B. Dittrich „Electrostatic properties of nine fluoroquinolone antibiotics derived directly from their crystal structure refinements“ Cryst. Eng. Comm. (2012), DOI: 10.1039/C1CE05966A

\section{Beiträge auf internationalen Konferenzen}

\section{Vorträge}

1. J. J. Holstein, P. Luger, C. Paulmann und B. Dittrich „Elektronendichteuntersuchung des Makrolidantibiotoikums Roxithromycin aus der Perspektive seiner pharmakologischen Aktivität: Vergleich der experimentellen Ladungsdichte mit Invariommodell und isoliertem Molekül“" 16. DGK Jahrestagung, Erlangen (2008) 


\section{Poster}

1. J. J. Holstein, D. Jayatilaka, S. Grabowsky, P. Luger und B. Dittrich „Achieving a reasonable parameterisation in multipole refinements: A study based on $R_{\text {free }}$, deformation density maps and residual density analysis“ 5th ECDM, Gravedona, Italy (2008)

2. J. J. Holstein, P. Luger, C. Paulmann und B. Dittrich „Validation of charge-density refinements and application to molecules of biological interest“ XXI Congress of the IUCr, Osaka, Japan (2008).

3. J. J. Holstein, C. B. Hübschle und B. Dittrich „Charge density study of ciprofloxacin · $\mathrm{HCl} \cdot 1.4 \mathrm{H}_{2} \mathrm{O}$ “ 17. DGK Jahrestagung, Hannover (2009)

4. C. B. Hübschle, J. J. Holstein und B. Dittrich „Enhancement of the Invariom Formalism“ Sagamore XVI, Santa Fee, USA (2009)

5. J. J. Holstein, C. B. Hübschle und B. Dittrich „Ab initio prediction of hydrogen-bond electron densities for crystal environments“ Sagamore XVI, Santa Fee, USA (2009)

6. B. Dittrich, E. Chen, J. J. Holstein, C. B. Hübschle, D. Jayatilaka „Crystalfield effects in L-homoserine from a simple point-charge model, from Hirshfeld-atom refinement and from a periodic B3LYP calculation“ GRC, Mount Holyoke, USA (2010).

7. J. J. Holstein, C. B. Hübschle und B. Dittrich „Automated charge-density refinement of heteroaromatic structures with the invariom database“ GRC, Mount Holyoke, USA (2010)

8. K. Pöpper, J. J. Holstein und B. Dittrich „A closer look at larger molecules: non-spherical refinement of thiostreptone“ ECM 26, Darmstadt (2010)

9. J. J. Holstein, C. B. Hübschle und B. Dittrich „Fast property comparison of fluoroquinolones with the revised invariom database“ ECM 26, Darmstadt (2010)

10. K. Pöpper, J. J. Holstein und B. Dittrich „Invariom modeling of DNAs“ ACA, New Orleans, USA (2011) 


\title{
Curriculum Vitae
}

\author{
Personal \\ Name: \\ Julian Jacob Holstein \\ Date of Birth: $\quad$ February 10th 1983 \\ Place of Birth: Berlin \\ Nationality: German \\ Marital Status: unmarried

\section{Education} \\ 1989 - $1993 \quad$ Süd-Grundschule, Berlin \\ $1993-2001 \quad$ Werner-von-Siemens-Gymnasium, Berlin \\ 06/2001 Allgemeine Hochschulreife, Abitur \\ Academic Career \\ 10/2001 - 09/2007 Chemistry student, Freie Universität Berlin \\ 02/2007 - 09/2007 Diploma thesis: "Experimental charge-density deter- \\ mination of erythromycin-type antibiotics" \\ advisor: Prof. Dr. Peter Luger \\ $11 / 2007$ - present $\quad$ Ph. D. student, Georg-August-Universität Göttingen \\ "Electron-density study of antibiotics with reference \\ to pharmacological efficacy" \\ advisor: Dr. habil. Birger Dittrich
}

Work Experience

11/2007 - present Scientific coworker, Georg-August-Universität Göttingen

04/2004-09/2007 Student research assistant, Freie Universität Berlin 